\title{
ARIZONA AND CALIFORNIA.
}

\section{TRIANGULATION STATIONS.}

Oatman special quadrangle, Kingman and Mohave City $30^{\prime}$ quadrangles.

MOHAVE COUNTY, ARIZONA; SAN BERNARDINO COUNTY, CALIFORNIA.

Points located by L. F. Biggs in 1916, based on Cone and Peak triangulation stations, on approximate North American datum.

\section{CONE NO. 2, MOHAVE COUNTY, ARIZ.}

A sharp rocky peak, well known as "The Boundary Cone," about 17 miles northeast of Needles, Calif. The road to Kingham via the Snowball mine passes by the base of the peak. To go to Cone from Needles, take road to Snow Ball mine, pass the Cone. Stop at the first big wash that the road crosses after passing the peak and go up the wash a short distance; the best and perhaps the only way up. Horses can not be taken much farther than the wash. There are several springs within 2 or 3 miles of the station. Plenty of water at Leland mine about 3 miles a little west of north of station.

Station mark: A bronze triangulation tablet wedged in solid rock, about 2 inches under ground, under center of a cairn 7 feet high.

[Latitude $34^{\circ} 59^{\prime} 01.39^{\prime \prime}$. Longitude $114^{\circ} 24^{\prime} 54.54^{\prime \prime}$.]

\begin{tabular}{|c|c|c|c|c|}
\hline To station- & Azimuth. & Back azimuth. & Distar & nce. \\
\hline 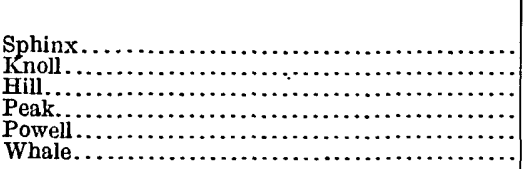 & \begin{tabular}{rcc}
\multicolumn{1}{c}{} & \multicolumn{1}{c}{$\prime \prime$} \\
333 & 32 & 18.97 \\
49 & 51 & 22.25 \\
69 & 08 & 44.90 \\
95 & 48 & 47.00 \\
356 & 11 & 33.97 \\
19 & 26 & 32.62
\end{tabular} & $\begin{array}{ccc}\circ & , & \prime \prime \\
153 & 36 & 43.44 \\
229 & 44 & 04.98 \\
248 & 59 & 57.08 \\
275 & 39 & 14.25 \\
176 & 12 & 24.71 \\
199 & 22 & 08.57\end{array}$ & $\begin{array}{r}\text { Log. meters. } \\
4.4215004 \\
4.4047806 \\
4.3985648 \\
4.4057480 \\
4.5321828 \\
4.5484345\end{array}$ & 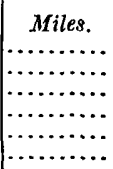 \\
\hline
\end{tabular}

FORT MOHAVE, MOHAVE COUNTY, ARIZ.

Center of large water tank at Fort Mohave.

[Latitude $35^{\circ} 02^{\prime} 39.450^{\prime \prime}$. Longitude $\left.114^{\circ} 37^{\prime} 19.712 .^{\prime \prime}\right]$

\begin{tabular}{|c|c|c|c|c|}
\hline To station- & Azimuth. & Back azimuth. & Dista & \\
\hline 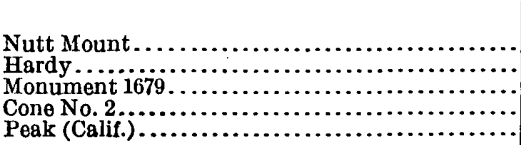 & \begin{tabular}{rcc}
\multicolumn{1}{c}{} &, & $\prime \prime$ \\
258 & 53 & 52.89 \\
264 & 31 & 39.96 \\
277 & 09 & 39.32 \\
289 & 31 & 09.76 \\
56 & 59 & 53.71
\end{tabular} & \begin{tabular}{rcc|}
\multicolumn{1}{c}{} & $\prime$ & $\prime \prime$ \\
79 & 03 & 10.35 \\
84 & 38 & 06.02 \\
97 & 16 & 21.01 \\
109 & 38 & 17.32 \\
236 & 57 & 28.21
\end{tabular} & $\begin{array}{r}\text { Log. meters. } \\
4.3987610 \\
4.2333038 \\
4.2523358 \\
4.3021699 \\
3.8844803\end{array}$ & $\begin{array}{r}\text { Miles. } \\
15.564 \\
10.633 \\
11.109 \\
12.460 \\
4.762\end{array}$ \\
\hline
\end{tabular}




\section{HARDY, MOHAVE COUNTY, ARIZ.}

On the highest point of Hardy Range, about 4 miles northwest of Oatman and about 0.5 mile west of the Jerome-Oatman mine. To reach station take road to JeromeOatman property, thence trail to mine, thence uphill.

Station mark: Tablet cemented in rock under center of cairn 7 feet high.

[Latitude $35^{\circ} 03^{\prime} 31.888^{\prime \prime}$ : Longitude $114^{\circ} 26^{\prime} 07.495^{\prime \prime}$.

\begin{tabular}{|c|c|c|c|c|}
\hline To station- & Azimuth. & Back azimuth. & Dist & nce. \\
\hline 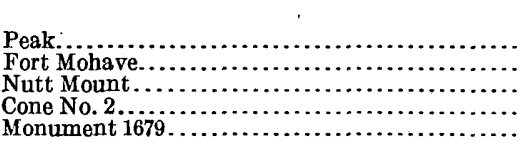 & $\begin{array}{rcc}8 & \prime & \prime \prime \\
76 & 12 & 24.55 \\
84 & 38 & 06.02 \\
247 & 10 & 50.94 \\
347 & 29 & 01.64 \\
349 & 45 & 23.76\end{array}$ & \begin{tabular}{rcc}
\multicolumn{1}{c}{} & $\prime$ & $\prime \prime$ \\
256 & 03 & 33.15 \\
264 & 31 & 39.96 \\
67 & 13 & 42.23 \\
167 & 29 & 43.51 \\
169 & 45 & 39.56
\end{tabular} & \begin{tabular}{r|} 
Log. meters. \\
4. 3832724 \\
4.2333038 \\
3.9133976 \\
3.9313729 \\
3.5937759
\end{tabular} & $\begin{array}{r}\text { Miles. } \\
15.018 \\
10.633 \\
5.090 \\
5.305 \\
2.439\end{array}$ \\
\hline
\end{tabular}

MONUMENT 1679, MOHAVE COUNTY, ARIZ.

A U. S. Mineral Location Monument No. 1679, on top of hill, just west of the old Leland mine.

Station mark: Not described.

Note.-The General Land Office tied to this station.

[Latitude $35^{\circ} 01^{\prime} 26.568^{\prime \prime}$. Longitude $114^{\circ} 25^{\prime} 39.966^{\prime \prime}$.]

\begin{tabular}{|c|c|c|c|c|}
\hline To station- & Azimuth. & Back azimuth. & \multicolumn{2}{|c|}{ Distance. } \\
\hline 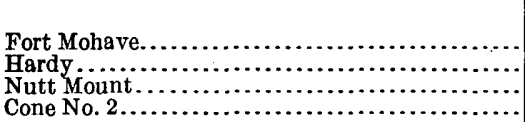 & \begin{tabular}{rcc}
\multicolumn{1}{c}{} & $\prime$ & $\prime \prime$ \\
97 & 16 & 21.02 \\
169 & 45 & 39.56 \\
224 & 14 & 15.95 \\
345 & 33 & 27.15
\end{tabular} & \begin{tabular}{rcc}
\multicolumn{1}{c}{} & \multicolumn{1}{c}{ ' } & \multicolumn{1}{c}{} \\
277 & 09 & 39.32 \\
349 & 45 & 23.76 \\
44 & 16 & 51.36 \\
165 & 33 & 53.21
\end{tabular} & $\begin{array}{r}\text { Log. meters. } \\
4.2523358 \\
3.5937759 \\
\text { 3.9922916 } \\
\text { 3. } 6646055\end{array}$ & $\begin{array}{r}\text { Miles. } \\
11.109 \\
2.439 \\
6.104 \\
2.870\end{array}$ \\
\hline
\end{tabular}

NUTT MOUNT, MOHAVE COUNTY, ARIZ.

On the most western of four prominent points of Mount Nutt. From Oatman take road to Gold Roads Summit, thence follow old trail around ridge to station. This is not a very good trail, but horses can be ridden over it. Another route is to take trail up canyon, follow pipe lines to end, thence keep main canyon following all left-hand canyons till the uppermost spring is reached, where there is a small concrete head gate and an old camp site, thence turn to left uphill and follow an old burro trail to top of ridge, thence go north to station. It is not advisable to take horses beyond spring. There is a higher point about 0.75 mile east of station, but not visible from Hardy.

Station mark: A bronze tablet.

Signal and reference mark: A cairn 7 feet high with drill hole under center, 2.5 feet toward Hardy from tablet.

[Latitude $35^{\circ} 05^{\prime} 14.885^{\prime \prime}$. Longitude $114^{\circ} 21^{\prime} 09.407^{\prime \prime}$.]

\begin{tabular}{|c|c|c|c|c|}
\hline To station- & Azimuth. & Back azimuth. & Distar & \\
\hline 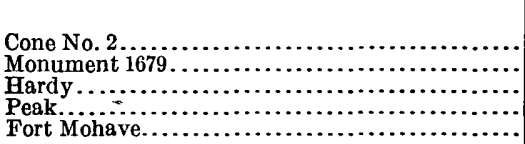 & $\begin{array}{lcc}\circ & \prime & \prime \prime \\
26 & 23 & 25.82 \\
44 & 16 & 51.36 \\
67 & 13 & 42.23 \\
73 & 58 & 22.97 \\
79 & 03 & 10.35\end{array}$ & $\begin{array}{ccc}\circ & \prime & \prime \prime \\
206 & 21 & 16.57 \\
224 & 14 & 15.95 \\
247 & 10 & 50.94 \\
253 & 46 & 40.19 \\
258 & 53 & 52.89\end{array}$ & $\begin{array}{l}\text { Log. meters. } \\
\text { 4. } 1087879 \\
\text { 3. } 9922916 \\
\text { 3. } 9133976 \\
\text { 4. } 5090153 \\
\text { 4. } 3987610\end{array}$ & $\begin{array}{r}\text { Miles. } \\
7.982 \\
6.104 \\
5.090 \\
20.062 \\
15.564\end{array}$ \\
\hline
\end{tabular}


PEAK, SAN BERNARDINO COUNTY, CALIF.

A U. S. Coast and Geodetic Survey triangulation station on the highest peak of the foothills, 2 miles due west of Von Schmidt.

Station mark: A shallow hole drilled in top of a large irregular mass of hard rock set in ground.

[Latitude $35^{\circ} 00^{\prime} 23.910^{\prime \prime}$. Longitude $114^{\circ} 41^{\prime} 33.211^{\prime \prime}$.]

\begin{tabular}{|c|c|c|c|c|}
\hline To station- & Azimuth. & Back azimuth. & Distal & nce. \\
\hline 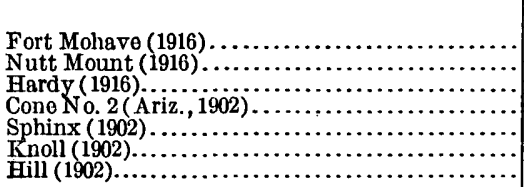 & $\begin{array}{ccc}\circ & \prime & \prime \prime \\
236 & 57 & 28.21 \\
253 & 46 & 40.19 \\
256 & 03 & 33.15 \\
275 & 39 & 14.25 \\
305 & 05 & 22.51 \\
342 & 31 & 30.78 \\
350 & 19 & 19.40\end{array}$ & $\begin{array}{rcc}\circ & \prime & \prime \prime \\
56 & 59 & 53.71 \\
73 & 58 & 22.97 \\
76 & 12 & 24.55 \\
95 & 48 & 47.00 \\
125 & 19 & 18.28 \\
162 & 33 & 45.08 \\
170 & 20 & 03.60\end{array}$ & \begin{tabular}{|c|} 
Log. meters. \\
3. 8844803 \\
4.5090153 \\
4. 3832724 \\
4. 4057480 \\
4. 6571412 \\
4. 2977878 \\
4.0663630
\end{tabular} & $\begin{array}{r}\text { Miles. } \\
4.762 \\
20.062 \\
15.018 \\
15.816 \\
\cdots . . . \% \\
\cdots\end{array}$ \\
\hline
\end{tabular}

\section{CALIFORNIA.}

\section{TRIANGULATION STATIONS.}

Burney, Dixie, Paynes Creek, Prattville, and Susanville quadrangles.

IASSEN, PLUMAS, ShaSTA, AND TEHAMA COUNTIES.

During the season of 1916 triangulation was extended over the above-named quadrangles by Charles $\mathrm{F}$. Urquhart.

Positions are on North American datum as derived from U. S. Coast and Geodetic Survey stations Bally, Round, and Lyons. Seventeen stations were occupied, 2 being primary stations, Lyons and Round, of the U. S. Coast and Geodetic Survey, and 13 points were located by intersections. Bally, a Coast and Geodetic Survey station, was resected on as a check. The final computations were made at the Sacramento, Calif., office.

ANTELOPE, LASSEN COUNTY, CALIF. (Not occupied.)

Highest point of prominent mountain, locally known as Antelope Peak or Campbell Peak. It is between the south end of Eagle Lake and Pine Creek Valley.

[Latitude $40^{\circ} 35^{\prime} 33.848^{\prime \prime}$. Longitudẹ $120^{\circ} 54^{\prime} 34.549^{\prime \prime}$.]

\begin{tabular}{|c|c|c|c|c|}
\hline To station- & Azimuth. & Back azimuth. & Distar & \\
\hline 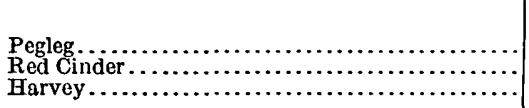 & $\begin{array}{rcc}\circ & \prime & \prime \prime \\
9 & 50 & 58.80 \\
69 & 32 & 09.01 \\
147 & 21 & 15.83\end{array}$ & $\begin{array}{ccc}\circ & \prime \prime & \prime \prime \\
189 & 49 & 20.42 \\
249 & 19 & 01.21 \\
327 & 16 & 22.49\end{array}$ & $\begin{array}{r}\text { Log. meters. } \\
\text { 4. } 3197279 \\
4.4837507 \\
4.2917915\end{array}$ & $\begin{array}{l}\text { Miles. } \\
12.974 \\
18.928 \\
12.166\end{array}$ \\
\hline
\end{tabular}

BALD MOUNTAIN, SHASTA COUNTY, CALIF.

On the highest point of Bald Mountain, a well-known peak, about 8 miles a little east of south of Fall River Mills, and immediately above and south of the Peterson and St. Johns ranches. The mountain is not bald as the name would suggest but was originally covered with pine timber from 1 to 3 feet on the stump. 'The timber was cut on highest point of mountain except a pine tree 40 or 50 feet high, which was trimmed and left for a signal. Timber was not cut on the east and west ends of mountain, but the signal tree is high enough to be seen over it. Animals can be ridden to 
summit; the easiest approach is from the south, though it was ascended from the northwest in 1916.

Station mark: A triangulation tablet set in large rock on highest part of mountain and instrument set over tablet for observation.

Signal is 10.49 meters in direction of Fall River Mills from triangulation station. Position of tablet computed.

[Latitude $40^{\circ} 54^{\prime} 03.984^{\prime \prime}$. Longitude $121^{\circ} 22^{\prime} 39.319^{\prime \prime}$.]

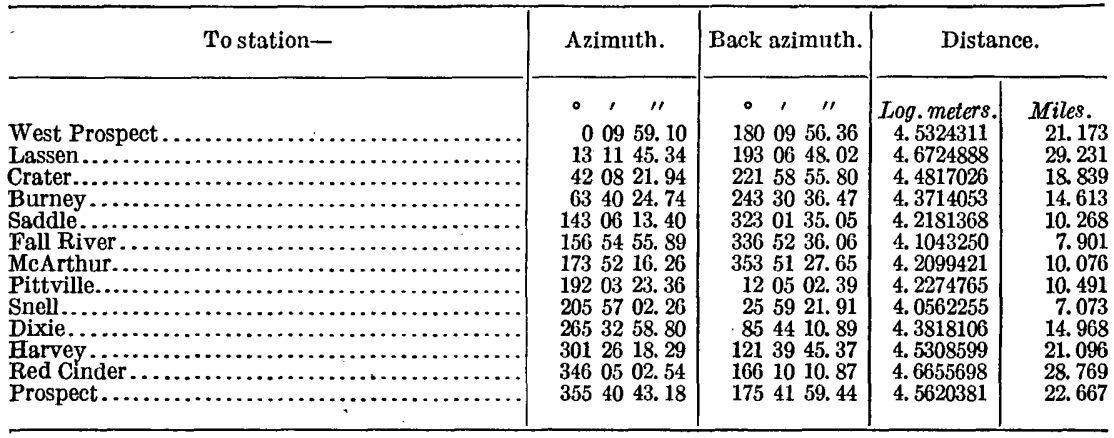

BALLY, SHASTA COUNTY, CALIF.

A station of the U. S. Coast and Geodetic Survey, on the more northern of the two main peaks on the summit of Bally Mountain, a prominent and well-known mountain about 15 miles by road west of Redding, Calif. The station mark, described below, is in the top of a rock on the north side of the most prominent group of rocks on the peak and 5 or 6 feet below the top of the group. Station mark: A $\frac{3}{4}$-inch copper bolt, 3 inches long, cemented into a drill hole in the rock, and directly above the bolt in the same drill hole is cemented an old-type station mark. A cross in the top of the copper bolt and another in the polished center of the disk mark the station.

Reference marks: A drill or punch hole in the top of a $\frac{3}{8}$-inch copper bolt, 3 inches long, which is leaded or cemented into a drill hole in a large rock with the top of the bolt flush with the surface. There are two such marks at the following distances and azimuths from the station: 29.69 meters, $126^{\circ} 59^{\prime}$; and 20.22 meters, $332^{\circ} 22^{\prime}$.

[Latitude $40^{\circ} 36^{\prime} 11.939^{\prime \prime}$. Longitude $122^{\circ} 39^{\prime} 00.370^{\prime \prime}$.]

\begin{tabular}{|c|c|c|c|c|}
\hline Tostation- & Azimuth. & Back azimuth. & Dista & ce. \\
\hline 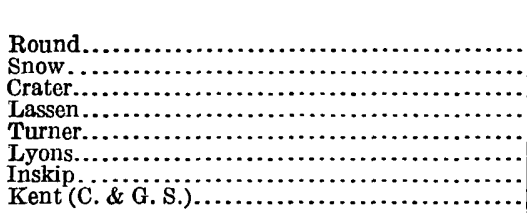 & \begin{tabular}{rcc}
\multicolumn{1}{c}{} & \multicolumn{1}{c}{$\prime \prime$} \\
248 & 47 & 16.01 \\
257 & 43 & 24.74 \\
262 & 45 & 54.49 \\
277 & 06 & 14.50 \\
290 & 42 & 40.44 \\
291 & 00 & 16.70 \\
295 & 34 & 26.22 \\
6 & 01 & 26.68
\end{tabular} & $\begin{array}{rcc}\circ & \prime & \prime \prime \\
69 & 14 & 22.18 \\
78 & 17 & 06.06 \\
83 & 26 & 14.42 \\
97 & 50 & 56.09 \\
111 & 22 & 55.57 \\
111 & 39 & 38.03 \\
116 & 01 & 59.83 \\
185 & 58 & 03.60\end{array}$ & $\begin{array}{r}\text { Log. meters. } \\
\text { 4. } 7972333 \\
4.8719614 \\
4.9440334 \\
4.9907799 \\
4.9730552 \\
4.9640937 \\
4.8238630 \\
4.8514233\end{array}$ & $\begin{array}{r}\text { Miles. } \\
38.957 \\
46.271 \\
54.624 \\
60.832 \\
58.399 \\
57.206 \\
41.420 \\
\end{array}$ \\
\hline
\end{tabular}

BROKEOFF, SHASTA-TEHAMA COUNTY LINE, CALIF. (Not occupied.)

A small rock cairn on highest point of Brokeoff Peak, a prominent mountain about 4 miles southwest of Lassen. 
[Latitude $40^{\circ} 26^{\prime} 45.716^{\prime \prime}$. Longitude $121^{\circ} 33^{\prime} 31.669^{\prime \prime}$.]

\begin{tabular}{|c|c|c|c|c|}
\hline To station- & Azimuth. & Back azimuth. & Distan & \\
\hline 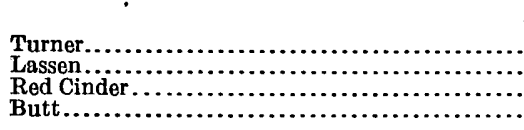 & $\begin{array}{ccc}\circ & \prime & \prime \prime \\
16 & 39 & 25.66 \\
224 & 22 & 13.87 \\
257 & 59 & 54.67 \\
329 & 44 & 20.35\end{array}$ & \begin{tabular}{rcc}
\multicolumn{1}{c}{} & $\prime$ & $\prime \prime$ \\
196 & 37 & 12.01 \\
44 & 24 & 21.32 \\
78 & 12 & 04.99 \\
149 & 51 & 04.40
\end{tabular} & $\begin{array}{r}\text { Log. meters. } \\
\text { 4. } 2304507 \\
3.8204344 \\
4.4327868 \\
4.4667596\end{array}$ & $\begin{array}{r}\text { Miles. } \\
10.563 \\
4.110 \\
16.832 \\
18.202\end{array}$ \\
\hline
\end{tabular}

BURNEY, SHASTA COUNTY, CALIF.

Station is on the highest point of Burney Butte, a well-known mountain about 6 miles south-southeast of Burney post office. No way was found to ride to summit of this peak. The shortest and best route is probably from Burney spring on south side of mountain. Road from Hat Creek to Tamarack Flat passes this spring.

Signal: A rock cairn 6 feet at base and 6 feet high.

Station mark: A bronze triangulation tablet set in rock under center of cairn.

[Latitude $40^{\circ} 48^{\prime} 24.889^{\prime \prime}$. Longitude $121^{\circ} 37^{\prime} 38.610^{\prime \prime}$.]

\begin{tabular}{|c|c|c|c|c|}
\hline Tostation- & Azimuth. & Back azimuth. & Dist & nce. \\
\hline 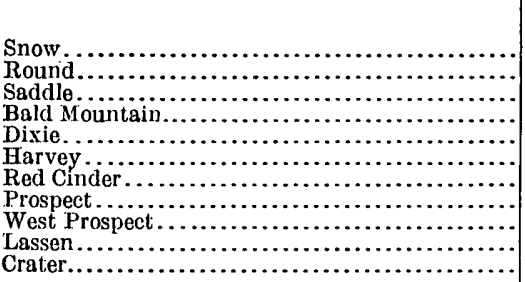 & \begin{tabular}{rcc}
\multicolumn{1}{c}{.} & $\prime$ & $\prime \prime$ \\
62 & 24 & 00.38 \\
89 & 47 & 24.34 \\
205 & 05 & 55.34 \\
243 & 30 & 36.47 \\
254 & 34 & 51.28 \\
278 & 06 & 41.95 \\
316 & 48 & 28.73 \\
317 & 16 & 21.80 \\
318 & 15 & 13.99 \\
343 & 32 & 28.70 \\
356 & 20 & 06.60
\end{tabular} & \begin{tabular}{rcc}
\multicolumn{1}{c}{} & \multicolumn{1}{c}{} & \multicolumn{1}{c}{} \\
242 & 17 & 41.34 \\
269 & 34 & 27.81 \\
25 & 11 & 06.23 \\
63 & 40 & 24.74 \\
74 & 55 & 51.10 \\
98 & 29 & 55.58 \\
137 & 03 & 22.63 \\
137 & 27 & 24.31 \\
138 & 24 & 57.70 \\
163 & 37 & 17.48 \\
176 & 20 & 28.06
\end{tabular} & $\begin{array}{r}\text { Log. meters. } \\
4.1865539 \\
4.4448735 \\
4.4174010 \\
4.3714053 \\
4.6697079 \\
4.7039186 \\
4.6740212 \\
4.5468666 \\
4.4998303 \\
4.5664436 \\
4.0818458\end{array}$ & $\begin{array}{r}\text { Miles. } \\
9.548 \\
17.307 \\
16.240 \\
14.613 \\
29.044 \\
31.424 \\
29.334 \\
21.889 \\
19.642 \\
22.898 \\
7.502\end{array}$ \\
\hline
\end{tabular}

BUTT, TEHAMA COUNTY, CALIF.

Signal is on highest point of Butt Mountain, a well-known mountain at source of East Fork of Deer Creek and just north of Butt Creek at a point 6 miles below the source. There is a very good road from Chester, on the Red Bluff and Susanville road, to Deer Creek Meadows, where there is good pasture. Distance from Chester to Deer Creek Meadows about 10 miles. There is another road (not much used) from Morgan Springs to Deer Creek Meadows. A trail crosses the ridge from Deer Creek Meadows to Butt Creek, which can be used to meadow on summit of ridge; cross lower end of this meadow and go up most northern ridge leading west from peak. Animals were taken to summit in 1916 .

This point was occupied by the Wheeler Survey in 1878, and a very good cairn was found standing. The new cairn was built in the same position.

Signal: A cairn about 8 feet high and 6 feet at base.

Station mark: A bronze triangulation tablet set under center of cairn.

[Latitude $40^{\circ} 13^{\prime} 04.955^{\prime \prime}$. Longitude $121^{\circ} 23^{\prime} 07.376^{\prime \prime}$.]

\begin{tabular}{|c|c|c|c|c|}
\hline Tostation- & Azimuth. & Back azimuth. & Dista & \\
\hline 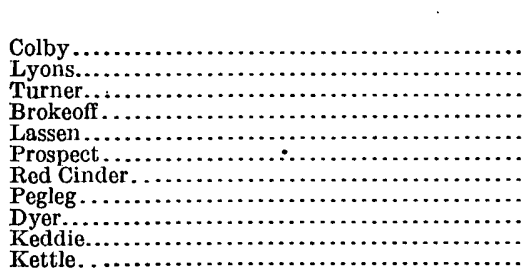 & $\begin{array}{rcc}\circ & \prime & \prime \prime \\
57 & 13 & 01.87 \\
113 & 21 & 37.23 \\
114 & 46 & 21.34 \\
149 & 51 & 04.40 \\
161 & 27 & 32.21 \\
184 & 56 & 06.51 \\
200 & 53 & 11.92 \\
240 & 09 & 17.40 \\
.265 & 21 & 44.10 \\
270 & 07 & 51.00 \\
277 & 42 & 06.41\end{array}$ & \begin{tabular}{rcc}
\multicolumn{1}{c}{} & $\prime$ & $\prime \prime$ \\
237 & 07 & 38.99 \\
293 & 11 & 46.79 \\
294 & 37 & 24.56 \\
329 & 44 & 20.35 \\
341 & 22 & 55.13 \\
4 & 57 & 40.42 \\
20 & 58 & 36.28 \\
60 & 26 & 07.53 \\
85 & 35 & 25.83 \\
90 & 24 & 01.20 \\
98 & 07 & 52.92
\end{tabular} & \begin{tabular}{|c|} 
Log. meters. \\
4.1488544 \\
4.3711283 \\
4.3345578 \\
4.4667596 \\
4.5009723 \\
4.5979369 \\
4.5196298 \\
4.6278285 \\
4.4796368 \\
4.5505945 \\
4.7577731
\end{tabular} & $\begin{array}{r}\text { Miles. } \\
8.754 \\
14.604 \\
13.425 \\
18.202 \\
19.693 \\
24.620 \\
20.558 \\
26.374 \\
18.749 \\
22.077 \\
35.573\end{array}$ \\
\hline
\end{tabular}


COLBY, TEHAMA COUNTY, CALIF. (Not occupied.)

Station is center of floor of tent used by U. S. Forest Service for lookout station.

Reference mark: A triangulation tablet set in rock 5.3 feet from southeast corner of tent, and 9.7 feet from northeast corner of tent.

[Latitude $40^{\circ} 08^{\prime} 57.337^{\prime \prime}$. Longitude $121^{\circ} 31^{\prime} 27.782^{\prime \prime}$.]

\begin{tabular}{|c|c|c|c|c|c|}
\hline To station- & . & Azimuth. & Back azimuth. & Distar & \\
\hline $\begin{array}{l}\text { Turner..... } \\
\text { Lassen..... } \\
\text { Butt...... }\end{array}$ & & $\begin{array}{ccc}\circ & \prime & \prime \prime \\
154 & 55 & 35.78 \\
182 & 35 & 38.89 \\
237 & 07 & 38.99\end{array}$ & \begin{tabular}{rcc}
\multicolumn{1}{c}{} & \multicolumn{1}{c}{$\quad \prime \prime$} \\
334 & 52 & 02.53 \\
2 & 36 & 25.79 \\
57 & 13 & 01.87
\end{tabular} & \begin{tabular}{|c|} 
Log. meters. \\
4. 2648901 \\
4. 5765499 \\
4.1488544
\end{tabular} & $\begin{array}{r}\text { Miles. } \\
11.435 \\
23.437 \\
8.754\end{array}$ \\
\hline
\end{tabular}

CONE, SHASTA COUNTY, CALIF. (Not occupied.)

The highest point of prominent peak on north side of Battle Creek and north of Mr. Long's ranch on Red Bluff and Manton road. About 7 miles west-southwest of Shingleton.

[Latitude $40^{\circ} 27^{\prime} 24.361^{\prime \prime}$. Longitude $121^{\circ} 59^{\prime} 56.731^{\prime \prime}$.]

\begin{tabular}{|c|c|c|c|c|}
\hline To station- & Azimuth. & Back azimuth. & Distan & \\
\hline 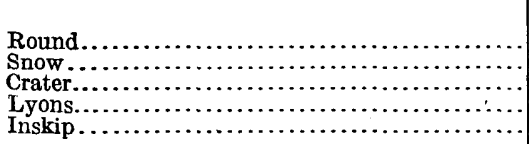 & $\begin{array}{ccc}\circ & \prime \prime \\
185 & 10 & 57.36 \\
209 & 13 & 45.66 \\
230 & 06 & 40.77 \\
299 & 16 & 42.51 \\
339 & 18 & 11.37\end{array}$ & $\begin{array}{rcc}\circ & \prime & \prime \prime \\
5 & 12 & 34.94 \\
29 & 21 & 58.77 \\
50 & 21 & 32.57 \\
119 & 30 & 41.94 \\
159 & 20 & 23.56\end{array}$ & \begin{tabular}{|c|} 
Log. meters. \\
4.5898083 \\
4.5611916 \\
4.6227303 \\
4.5450833 \\
4.1342037
\end{tabular} & $\begin{array}{r}\text { Miles. } \\
24.163 \\
22.623 \\
26.066 \\
21.799 \\
8.463\end{array}$ \\
\hline
\end{tabular}

COYOTE, LASSEN COUNTY, CALIF. (Not occupied.)

Station is a rock cairn on prominent mountain on east side of Mountain Meadows and 12 or 15 miles east of Westwood.

[Latitude $40^{\circ} 17^{\prime} 25.131^{\prime \prime}$. Longitude $120^{\circ} 49^{\prime} 53.496^{\prime \prime}$.]

\begin{tabular}{|c|c|c|c|c|}
\hline To station- & Azimuth. & Back azimuth. & Dista & \\
\hline 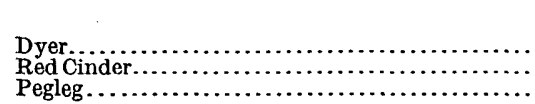 & $\begin{array}{ccc}\circ & , & \prime \prime \\
71 & 44 & 43.97 \\
123 & 08 & 45.80 \\
141 & 55 & 02.34\end{array}$ & $\begin{array}{ccc}\circ & \prime & \prime \prime \\
251 & 36 & 57.62 \\
302 & 52 & 38.30 \\
321 & 50 & 22.30\end{array}$ & \begin{tabular}{|r|} 
Log. meters. \\
4. 2542995 \\
4.6231468 \\
4.2184094
\end{tabular} & $\begin{array}{l}\text { Miles. } \\
11.160 \\
26.091 \\
10.274\end{array}$ \\
\hline
\end{tabular}

\section{CRATER, SHASTA COUNTY, CALIF.}

Station is on the highest point of high mountain locally known as Magee Peak. The old Geological Survey map gives this point as Crater Peak, and the Coast Survey used the same name in 1904, consequently it has been retained. Animals can be ridden to within about 400 feet (elevation) of the summit on north side of mountain starting from Tamarack Flat. The U. S. Forest Service has a lookout on this mountain but no cabin has been built.

Signal: A rock cairn 6 feet at base and 7 feet high with small tree in center.

Station mark: A bronze triangulation tablet set in solid rock under center of cairn. 
[Latitude $40^{\circ} 41^{\prime} 54.271^{\prime \prime}$. Longitude $121^{\circ} 37^{\prime} 05.738^{\prime \prime}$.]

\begin{tabular}{|c|c|c|c|c|}
\hline To station- & Azimuth. & Back azimuth. & Dist & nce. \\
\hline 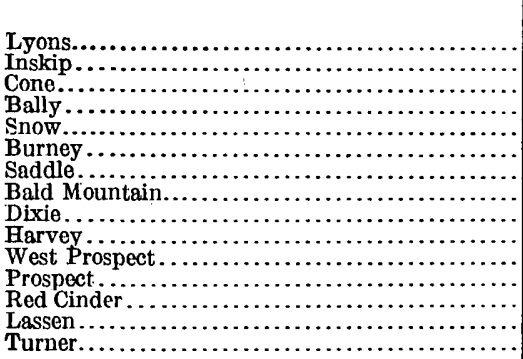 & \begin{tabular}{rcc}
$\circ$ & \multicolumn{1}{c}{} & \multicolumn{1}{c}{} \\
2 & 18 & 38.79 \\
34 & 52 & 20.05 \\
50 & 21 & 32.57 \\
83 & 26 & 14.42 \\
108 & 55 & 10.59 \\
176 & 20 & 28.06 \\
196 & 06 & 58.37 \\
221 & 58 & 55.80 \\
241 & 04 & 40.38 \\
264 & 18 & 52.05 \\
299 & 38 & 35.70 \\
300 & 52 & 43.35 \\
305 & 21 & 04.94 \\
337 & 27 & 40.91 \\
359 & 45 & 49.27
\end{tabular} & \begin{tabular}{rcc}
\multicolumn{1}{c}{} & $\prime$ & $\prime \prime$ \\
182 & 17 & 49.91 \\
214 & 39 & 41.81 \\
230 & 06 & 40.77 \\
262 & 45 & 54.49 \\
288 & 48 & 30.52 \\
356 & 20 & 06.60 \\
16 & 11 & 47.41 \\
42 & 08 & 21.94 \\
61 & 25 & 17.34 \\
84 & 41 & 42.70 \\
119 & 47 & 57.35 \\
121 & 03 & 23.72 \\
125 & 35 & 36.45 \\
157 & 32 & 07.98 \\
179 & 45 & 54.30
\end{tabular} & $\begin{array}{r}\text { Log. meters. } \\
4.6443176 \\
4.6828360 \\
4.6227303 \\
4.9440334 \\
4.1821344 \\
4.0818458 \\
4.5703853 \\
4.4817026 \\
4.7041650 \\
4.6949513 \\
4.3678633 \\
4.4305236 \\
4.5873406 \\
4.4017203 \\
4.6465358\end{array}$ & $\begin{array}{r}\text { Miles. } \\
27.395 \\
29.935 \\
26.066 \\
54.624 \\
9.451 \\
7.502 \\
23.107 \\
18.839 \\
31.442 \\
30.782 \\
14.495 \\
16.745 \\
24.027 \\
15.670 \\
27.535\end{array}$ \\
\hline
\end{tabular}

DE WITT, TEHAMA COUNTY, CALIF.

Station is on a point well known as De Witt Peak, on a ridge between Antelope and Mill creeks. It is about 7 miles east of Mr. D. B. Lyon's ranch in the Sacramento Valley, 7 or 8 miles east of Los Molinos on the Southern Pacific R. R. There is a road from Lyon's ranch to foot of hill.

Signal: A cairn 6 feet at base and 6 feet high with tree in center.

Station mark: A drill hole in solid rock under center of cairn.

[Latitude $40^{\circ} 09^{\prime} 27.630^{\prime \prime}$. Longitude $121^{\circ} 56^{\prime} 46.127 .^{\prime \prime}$ ]

\begin{tabular}{|c|c|c|c|c|}
\hline Tostation- & Azimuth. & Back azimuth. & Distar & \\
\hline 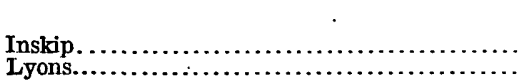 & $\begin{array}{ccc}\circ & \prime & \prime \prime \\
180 & 52 & 56.22 \\
238 & 25 & 51.97\end{array}$ & $\begin{array}{rcc}\circ & \prime & \prime \prime \\
0 & 53 & 4.85 \\
58 & 37 & 45.72\end{array}$ & $\begin{array}{r}\text { Log. meters. } \\
\text { 4. } 3111148 \\
4.4801773\end{array}$ & $\begin{array}{r}\text { Miles. } \\
12.719 \\
19.034\end{array}$ \\
\hline
\end{tabular}

DIXIE, LASSEN COUNTY, CALIF.

Station is on a prominent hill about 2 or 3 miles north of the Clarke \& Cox ranch in Dixie Valley; a well-known valley about 25 miles east-southeast of Fall River Mills. There is a good road from the latter place to the valley. The hill was covered with mahogany trees, which were cut down except one.

Signal: A mahogany tree standing alone, can be seen from the Clarke \& Cox ranch. Station mark: A bronze triangulation tablet set in rock under center of signal tree. Position of tablet computed.

[Latitude $40^{\circ} 55^{\prime} 03.309^{\prime \prime}$. Longitude $121^{\circ} 05^{\prime} 33.022^{\prime \prime}$.]

\begin{tabular}{|c|c|c|c|c|}
\hline Tostation- & Azimuth. & Back azimuth. & Distar & \\
\hline $\begin{array}{l}\text { Crater } \\
\begin{array}{l}\text { Burney } \\
\text { Bald Mountain }\end{array} \\
\text { Saddle } \ldots \ldots \ldots \ldots \ldots \ldots \ldots \ldots\end{array}$ & $\begin{array}{rcc}\circ & \prime & \prime \prime \\
61 & 25 & 17.34 \\
74 & 55 & 51.10 \\
85 & 44 & 10.89 \\
108 & 40 & 23.36 \\
345 & 59 & 34.01\end{array}$ & $\begin{array}{ccc}\circ & \prime & \prime \prime \\
241 & 04 & 40.38 \\
254 & 34 & 51.28 \\
265 & 32 & 58.80 \\
288 & 24 & 32.07 \\
166 & 01 & 50.22\end{array}$ & $\begin{array}{r}\text { Log. meters. } \\
\text { 4. } 7041650 \\
4.6697079 \\
4.3818106 \\
4.5536808 \\
4.3052244\end{array}$ & $\begin{array}{r}\text { Miles. } \\
31.442 \\
29.044 \\
14.968 \\
22.235 \\
12.548\end{array}$ \\
\hline
\end{tabular}

DYER, IASSEN AND PLUMAS COUNTY LINE, CALIF.

Station is on bald point at northwest end of ridge that has Dyer Peak at one end and Keddie Peak at the other. It is about 5 miles a little west of south from Westwood and about 5 miles east of Lake Almanor and is owned by the Great Western Power Co. It can be easily reached on horseback from almost any direction, but the top 
of ridge between it and Keddie Peak is rather rough. This point was probably used by the Wheeler Survey in 1878, but no description appears in the report. A very good rock cairn was found standing, and station was rebuilt on exactly the same spot.

Signal: A rock cairn about 6 feet at base and 6 feet high having a fir tree in center Station mark: A bronze triangulation tablet set in solid rock under center of cairn ${ }^{\circ}$

[Latitude $40^{\circ} 14^{\prime} 22.114^{\prime \prime}$. Longitude $121^{\circ} 01^{\prime} 55.040^{\prime \prime}$.]

\begin{tabular}{|c|c|c|c|c|}
\hline To station- & Aximuth. & Back azimuth. & Dist & nce. \\
\hline 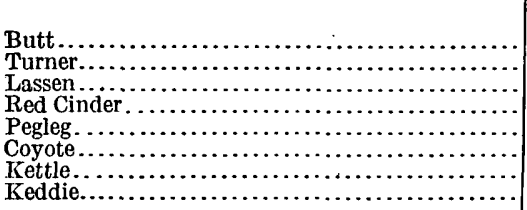 & \begin{tabular}{rcc}
\multicolumn{1}{c}{} & \multicolumn{1}{c}{$\prime \prime$} \\
85 & 35 & 25.83 \\
97 & 48 & 31.95 \\
124 & 44 & 25.11 \\
147 & 31 & 32.92 \\
200 & 03 & 58.81 \\
251 & 36 & 57.62 \\
291 & 00 & 05.14 \\
295 & 02 & 00.65
\end{tabular} & \begin{tabular}{rcc}
\multicolumn{1}{c}{$\circ$} & $\prime$ & $\prime \prime$ \\
265 & 21 & 44.10 \\
277 & 25 & 52.63 \\
304 & 26 & 03.94 \\
327 & 23 & 13.26 \\
20 & 07 & 05.83 \\
71 & 44 & 43.97 \\
111 & 12 & 10.86 \\
115 & 04 & 29.35
\end{tabular} & $\begin{array}{r}\text { Log. meters. } \\
4.4796368 \\
4.7001031 \\
4.6877907 \\
4.5293560 \\
4.2980235 \\
4.2542995 \\
4.4550164 \\
\text { 3. } 7787835\end{array}$ & $\begin{array}{r}\text { Miles. } \\
18.749 \\
31.150 \\
30.279 \\
21.024 \\
12.342 \\
11.160 \\
17.716 \\
3.734\end{array}$ \\
\hline
\end{tabular}

FALL RIVER, SHASTA COUNTY, CALIF. (Not occupied. No check.)

Station mark: Steeple on church in town of Fall River Mills.

[Latitude $41^{\circ} 00^{\prime} 23.120^{\prime \prime}$. Longitude $121^{\circ} 26^{\prime} 12.655^{\prime \prime}$.]

\begin{tabular}{|c|c|c|c|c|}
\hline Tostation- & Azimuth. & Back azimuth. & Distance. & \\
\hline 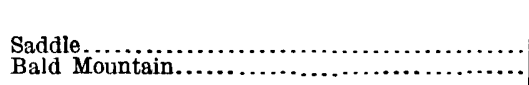 & $\begin{array}{ccc}\circ & \prime & \prime \prime \\
107 & 03 & 33.63 \\
336 & 52 & 36.06\end{array}$ & $\begin{array}{ccc}\circ & \prime & \prime \prime \\
287 & 01 & 14.98 \\
156 & 54 & 55.89\end{array}$ & $\begin{array}{r}\text { Log. meters. } \\
3.7129613 \\
4.1043250\end{array}$ & $\begin{array}{l}\text { Miles. } \\
3.209 \\
7.901\end{array}$ \\
\hline
\end{tabular}

HARVEY, LASSEN COUNTY, CALIF.

On the highest point of Harvey Mountain, which is on the northeast side of Harvey Valley. The greater part of the valley is owned by Mr. D. B. Lyon, but the Forest Service has a small pasture at the Harvey Valley Station, which is in the southeast end of the valley and is 5 miles south of triangulation station. There is a good trail from the Forest Service station to summit of mountain, which is a lookout station, but there is no house on summit. The Forest Service station is about 32 miles from Westwood by road. The valley can be reached by road from Fall River Mills, also from Dixie Valley, but the latter road is not much better than a pack trail. The mountain is covered with timber except the rocky peak on summit, and there are a few tall trees on its side reaching about as high as summit of mountain.

Signal: A cairn 6 feet at base and 6 feet high.

Station mark: A nail set in solid rock under center of cairn.

There is a small fir tree over center of mark; position of mark computed.

[Latitude $40^{\circ} 44^{\prime} 28.067^{\prime \prime}$. Longitude $121^{\circ} 02^{\prime} 04.698^{\prime \prime}$.]

\begin{tabular}{|c|c|c|c|c|}
\hline To station- & Azimuth. & Back azimuth. & Dista & \\
\hline 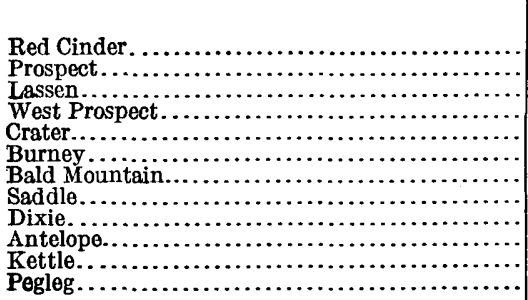 & $\begin{array}{rcc}\circ & \prime \prime & \prime \prime \\
33 & 26 & 34.22 \\
54 & 46 & 30.22 \\
54 & 56 & 41.21 \\
60 & 50 & 31.38 \\
84 & 41 & 42.70 \\
98 & 29 & 55.58 \\
121 & 39 & 45.37 \\
128 & 42 & 56.44 \\
166 & 01 & 50.22 \\
327 & 16 & 22.49 \\
337 & 50 & 29.75 \\
349 & 14 & 12.85\end{array}$ & $\begin{array}{ccc}\circ & \prime \prime \\
213 & 18 & 18.28 \\
234 & 34 & 21.96 \\
234 & 38 & 20.66 \\
240 & 37 & 04.07 \\
264 & 18 & 52.05 \\
278 & 06 & 41.95 \\
301 & 26 & 18.29 \\
308 & 24 & 50.49 \\
345 & 59 & 34.01 \\
147 & 21 & 15.83 \\
158 & 02 & 45.49 \\
169 & 17 & 27.11\end{array}$ & $\begin{array}{r}\text { Log. meters. } \\
4.5125597 \\
4.5076492 \\
4.6869818 \\
4.5231887 \\
4.6949513 \\
4.7039186 \\
4.5308599 \\
4.6962252 \\
4.3052244 \\
4.2917915 \\
4.8523500 \\
4.5764747\end{array}$ & $\begin{array}{r}\text { Miles. } \\
20.226 \\
19.999 \\
30.223 \\
20.727 \\
30.782 \\
31.424 \\
21.096 \\
30.873 \\
12.548 \\
12.166 \\
44.228 \\
23.433\end{array}$ \\
\hline
\end{tabular}


INSKIP, TEHAMA COUNTY, CALIF.

Station is on highest point of Inskip Hill, a well-known point about 1 mile west of Paynes Creek post office on the Red Bluff and Susanville road. It can be easily reached with saddle animals.

Signal: A rock cairn 6 feet at base and 6 feet high with tree in center.

Station mark: A bronze triangulation tablet under center of cairn.

[Latitude $40^{\circ} 20^{\prime} 31.214^{\prime \prime}$. Longitude $121^{\circ} 56^{\prime} 32.772^{\prime \prime}$.]

\begin{tabular}{|c|c|c|c|c|}
\hline To station- & Azimuth. & Back azimuth. & Distar & \\
\hline 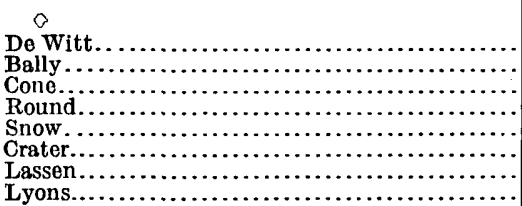 & $\begin{array}{rcc}\circ & \prime & \prime \prime \\
0 & 53 & 04.85 \\
116 & 01 & 59.83 \\
159 & 20 & 23.56 \\
178 & 35 & 19.06 \\
196 & 16 & 28.31 \\
214 & 39 & 41.81 \\
246 & 13 & 15.08 \\
279 & 45 & 10.37\end{array}$ & \begin{tabular}{rcc}
\multicolumn{1}{c}{} & $\prime$ & $\prime \prime$ \\
180 & 52 & 56.22 \\
295 & 34 & 26.22 \\
339 & 18 & 11.37 \\
358 & 34 & 43.87 \\
16 & 22 & 28.27 \\
34 & 52 & 20.05 \\
66 & 30 & 17.79 \\
99 & 56 & 56.83
\end{tabular} & \begin{tabular}{r|} 
Log. meters. \\
4.3111148 \\
4.8238630 \\
4.1342037 \\
4.7116944 \\
4.6661722 \\
4.6828360 \\
4.6085032 \\
4.4176646
\end{tabular} & $\begin{array}{r}\text { Miles. } \\
12.719 \\
41.420 \\
8.463 \\
31.992 \\
28.809 \\
29.935 \\
25.226 \\
16.256\end{array}$ \\
\hline
\end{tabular}

KEDDIE, LASSEN-PLUMAS COUNTY LINE, CALIF. (Not occupied.)

The highest point of Keddie Peak, which is about 5 miles north-northeast of Greenville post office and on south side of Mountain Meadows.

[Latitude $40^{\circ} 12^{\prime} 59.616^{\prime \prime}$. Longitude $120^{\circ} 58^{\prime} 04.799^{\prime \prime}$.

\begin{tabular}{|c|c|c|c|c|}
\hline Tostation- & Azimuth. & Back azimuth. & \multicolumn{2}{|c|}{ Distance. } \\
\hline 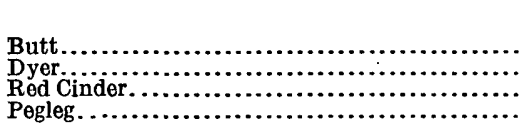 & $\begin{array}{rcc}\circ & \prime \prime & \prime \prime \\
90 & 24 & 01.20 \\
115 & 04 & 29.35 \\
142 & 49 & 26.65 \\
183 & 44 & 25.62\end{array}$ & \begin{tabular}{rcc}
\multicolumn{1}{c}{} & $\prime$ & $\prime \prime$ \\
270 & 07 & 51.00 \\
295 & 02 & 00.65 \\
322 & 38 & 38.02 \\
3 & 45 & 03.64
\end{tabular} & $\begin{array}{r}\text { Log. meters. } \\
4.5505945 \\
3.7787835 \\
4.5914659 \\
4.3272192\end{array}$ & $\begin{array}{r}\text { Miles. } \\
22.077 \\
3.734 \\
24.256 \\
13.200\end{array}$ \\
\hline
\end{tabular}

KETTLE, PLUMAS COUNTY, CALIF. (Not occupled.)

A Forest Service cabin on Kettle Rock, about 10 miles northeast of Taylorsville.

[Latitude $40^{\circ} 08^{\prime} 49.304^{\prime \prime}$. Longitude $120^{\circ} 43^{\prime} 10.531^{\prime \prime}$.]

\begin{tabular}{|c|c|c|c|c|}
\hline Tostation- & Azimuth. & Back azimuth. & Dist & nce. \\
\hline 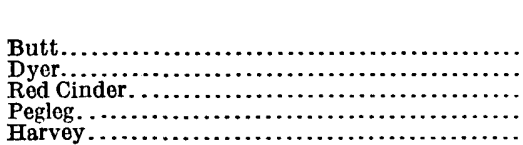 & $\begin{array}{rcc}\circ & \prime & \prime \prime \\
98 & 07 & 52.92 \\
111 & 12 & 10.86 \\
131 & 04 & 45.22 \\
145 & 45 & 32.28 \\
158 & 02 & 45.49\end{array}$ & $\begin{array}{ccc}\circ & \prime & \prime \prime \\
277 & 42 & 06.41 \\
291 & 00 & 05.14 \\
310 & 44 & 18.39 \\
325 & 36 & 32.14 \\
337 & 50 & 29.75\end{array}$ & \begin{tabular}{|r|} 
Log. meters. \\
4.7577731 \\
4.4550164 \\
4.7725326 \\
4.5442227 \\
4.8523500
\end{tabular} & $\begin{array}{r}\text { Miles. } \\
35.573 \\
17.716 \\
36.803 \\
21.756 \\
44.228\end{array}$ \\
\hline
\end{tabular}

IASSEN, SHASTA COUNTY, CAIIF.

Station is the highest point of Lassen Peak. It can be reached from Mineral, Morgan, or Drakesbad by road and trail to timber line on the south side, thence by trail to summit. About 2,000 feet above timber line is rather steep to ride an animal, but a pack animal with about 90 pounds of instruments was taken to the summit. A constant issue of steam from the mountain interferes with view to the northwest, but it can be seen through from time to time. The part of the mountain from which steam, ashes, and sometimes lava issue is about 200 yards northwest of station. The position of the tablet was computed.

Signal: A cairn 6 feet at base and 5 feet high.

Station mark: A triangulation tablet set in solid rock under center of cairn. 
[Latitude $40^{\circ} 29^{\prime} 18.939^{\prime \prime}$. Longitude $121^{\circ} 30^{\prime} 15.296^{\prime \prime}$.

\begin{tabular}{|c|c|c|c|c|}
\hline To station- & Azimuth. & Back azimuth. & \multicolumn{2}{|c|}{ Distance. } \\
\hline O- 1 - & - ' " & $\circ \quad$ ' "' & Log. meters. & Miles. \\
\hline Colby...... & 23625.79 & 1823538.89 & 4.5765499 & 23.437 \\
\hline Turner...... & 242124.63 & 2041703.66 & 4.3628774 & 14. 329 \\
\hline 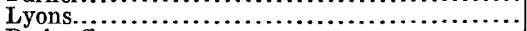 & 285625.67 & 2085110.91 & 4. 3748371 & 14. 729 \\
\hline Brokeoff. & 442421.32 & 2242213.87 & 3.8204344 & 4.110 \\
\hline Inskip.............. & 663017.79 & 2461315.08 & 4. 6085032 & 25.226 \\
\hline Bally ............... & 975056.09 & 2770614.50 & 4. 9907799 & 60.832 \\
\hline Snow................. & 1393801.75 & 3192655.34 & 4. 5691893 & 23.043 \\
\hline 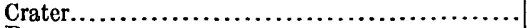 & 1573207.98 & 3372740.91 & 4.4017203 & 15.670 \\
\hline Burney............ & $163 \quad 37 \quad 17.48$ & 3433228.70 & 4.5664436 & 22.898 \\
\hline Bald Mountain.... & 1930648.02 & 131145.34 & 4. 6724888 & 29.231 \\
\hline West Prospect..... & $22208 \quad 17.02$ & 421310.68 & 4. 1995763 & 6838 \\
\hline Harvey............ & 2343820.66 & 545641.21 & 4.6869818 & 30.223 \\
\hline Prospect........ & 2345815.90 & 550428.17 & 4. 2162828 & 10.224 \\
\hline Red Cinder...... & 2673955.94 & 874959.07 & 4.3402539 & 13.602 \\
\hline Pegleg........... & 2804155.97 & 1010326.53 & 4.6788424 & 29.661 \\
\hline 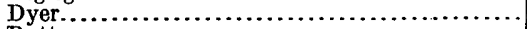 & 3042603.94 & 1244425.11 & 4.6877907 & 30.279 \\
\hline 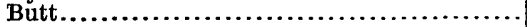 & 3412255.13 & 1612732.21 & 4.5009723 & 19.693 \\
\hline
\end{tabular}

IYONS, TEHAMA COUNTY, CALIF.

A.station of the U. S. Coast and Geodetic Survey about 5 miles east of Lyonsville post office, on a ridge known locally as Bald Hill, about 300 yards from the summit of the ridge, on the shoulder extending toward Lyonsville, and in the middle of a large group of boulders. A lone fir tree stands about 50 meters south by east from the station. The best route to the station is from Mineral post office.

Station mark: A $\frac{3}{5}$-inch copper bolt 3 inches long, cemented into a drill hole in the rock, and directly above the bolt in the same drill hole is cemented an old-type station mark. A cross in the top of the copper bolt and another in the polished center of the disk mark the station.

Reference marks: A drill or punch hole in the top of a $\frac{3}{8}$-inch copper bolt 3 inches long, which is leaded or cemented into a drill hole in large rock with the top of the bolt flush with the surface; marks are at the following distances and azimuths from the station: 29.69 meters, $126^{\circ} 59^{\prime}$; and 20.22 meters, $332^{\circ} 22^{\prime}$.

[Latitude $40^{\circ} 18^{\prime} 06.100^{\prime \prime}$. Longitude $121^{\circ} 38^{\prime} 21.008^{\prime \prime}$.]

\begin{tabular}{|c|c|c|c|c|}
\hline To station- & Azimuth. & Back azimuth. & Distar & nce. \\
\hline 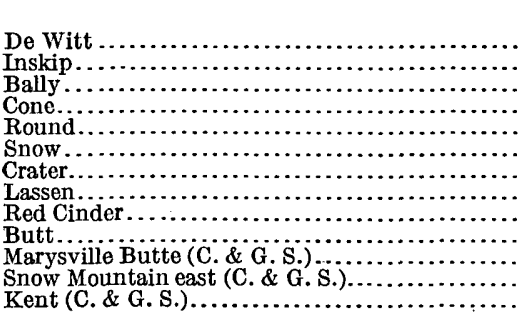 & \begin{tabular}{rcc}
\multicolumn{1}{c}{} & $\prime$ & $\prime \prime$ \\
58 & 37 & 45.72 \\
99 & 56 & 56.83 \\
111 & 39 & 38.03 \\
119 & 30 & 41.94 \\
154 & 22 & 27.03 \\
165 & 32 & 56.28 \\
182 & 17 & 49.91 \\
208 & 51 & 10.91 \\
236 & 55 & 59.39 \\
293 & 11 & 46.79 \\
7 & 18 & 50.85 \\
43 & 24 & 27.26 \\
68 & 42 & 27.63
\end{tabular} & 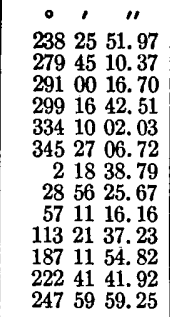 & $\begin{array}{r}\text { Log. meters. } \\
4.4861773 \\
4.4176646 \\
4.9640937 \\
4.5450833 \\
4.7931190 \\
4.7039865 \\
4.6443176 \\
4.3748371 \\
4.5993128 \\
4.3711283 \\
5.0885296 \\
5.1444074 \\
5.0030155\end{array}$ & $\begin{array}{r}\text { Miles. } \\
19.034 \\
16.256 \\
57.206 \\
21.799 \\
38.590 \\
31.429 \\
27.395 \\
14.729 \\
24.698 \\
14.604 \\
\ldots \ldots . . . \\
\ldots \ldots \ldots . . . \\
\ldots \ldots . . .\end{array}$ \\
\hline
\end{tabular}

M'ARTHUR, SHASTA COUNTY, CALIF. (No check. Not occupied.)

Station mark: Cupola on school building on south side of town of McArthur.

[Latitude $41^{\circ} 02^{\prime} 46.651^{\prime \prime}$. Longitude $121^{\circ} 23^{\prime} 53.448^{\prime \prime}$.]

\begin{tabular}{|c|c|c|c|c|}
\hline To station- & Azimuth. & Back azimuth. & Distan & ce. \\
\hline $\begin{array}{l}\text { Saddle } \ldots \ldots \ldots \ldots \ldots \\
\text { Bald Mountain } \ldots \ldots \ldots \ldots \ldots \ldots \ldots\end{array}$ & \begin{tabular}{ccc}
\multicolumn{1}{c}{ ' } & $\prime$ & $\prime \prime$ \\
70 & 26 & 16.09 \\
353 & 51 & 27.65
\end{tabular} & $\begin{array}{ccc}\circ & \prime & \prime \prime \\
250 & 22 & 26.00 \\
173 & 52 & 16.26\end{array}$ & $\begin{array}{c}\text { Log. meters. } \\
3.9390518 \\
4.2099421\end{array}$ & $\begin{array}{r}\text { Miles. } \\
5.400 \\
10.076\end{array}$ \\
\hline
\end{tabular}


PEgLEG, LASSEN COUNTY, CALIF.

Station is on the highest point of Pegleg Mountain, a well-known peak about 2.5 miles south of Westwood Junction on the Southern Pacific R. R., 11 miles north of Westwood. The U. S. Forest Service has a lookout station on the highest point of mountain, and the cabin, 10 feet square and about 15 feet high, was used for a signal.

Reference mark: A bronze triangulation tablet set in solid rock, 4.02 meters a little east of south of cabin, and instrument placed over tablet. True azimuth cabin to tablet $342^{\circ} 52^{\prime}$.

Position of cabin computed.

[Latitude $40^{\circ} 24^{\prime} 26.872^{\prime \prime}$. Longitude $120^{\circ} 57^{\prime} 06.027^{\prime \prime}$.]

\begin{tabular}{|c|c|c|c|c|}
\hline To station- & Azimuth. & Back azimuth. & Dist & nee. \\
\hline 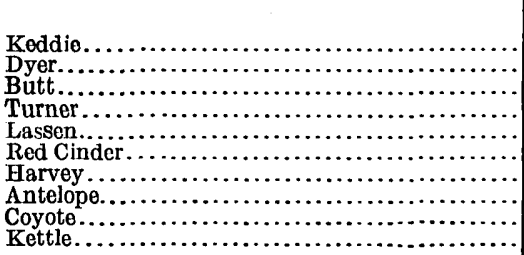 & $\begin{array}{rcc}\bullet & \prime & \prime \prime \\
3 & 45 & 03.64 \\
20 & 07 & 05.83 \\
60 & 26 & 07.53 \\
78 & 12 & 28.55 \\
101 & 03 & 26.53 \\
111 & 38 & 43.97 \\
169 & 17 & 27.11 \\
189 & 49 & 20.42 \\
321 & 50 & 22.30 \\
325 & 36 & 32.14\end{array}$ & \begin{tabular}{rcc}
\multicolumn{1}{c}{} & $\prime$ & $\prime \prime$ \\
183 & 44 & 25.62 \\
200 & 03 & 58.81 \\
240 & 09 & 17.40 \\
257 & 46 & 39.74 \\
280 & 41 & 55.97 \\
291 & 27 & 15.94 \\
349 & 14 & 12.85 \\
9 & 50 & 58.80 \\
141 & 55 & 02.34 \\
145 & 45 & 32.28
\end{tabular} & \begin{tabular}{r|} 
Log. meters. \\
4. 3272192 \\
4. 2980235 \\
4. 6278285 \\
4.7612451 \\
4. 6788424 \\
4.4292118 \\
4.5764747 \\
4.3197279 \\
4.2184094 \\
4.5442227
\end{tabular} & $\begin{array}{r}\text { Miles. } \\
13.200 \\
12.342 \\
26.374 \\
35.859 \\
29.661 \\
16.694 \\
23.433 \\
12.974 \\
10.274 \\
21.756\end{array}$ \\
\hline
\end{tabular}

PITTVILLE, Shasta COUNTY, CALIF. (Not occupied. No check.)

Station mark: Steeple on church at Pittville post office.

[Latitude $41^{\circ} 02^{\prime} 59.220^{\prime \prime}$. Longitude $121^{\circ} 20^{\prime} 08.309^{\prime \prime}$.]

\begin{tabular}{|c|c|c|c|c|}
\hline Tostation- & Azimuth. & Back azimuth. & Distan & \\
\hline $\begin{array}{l}\text { Bald Mountain............. } \\
\text { Saddle................... }\end{array}$ & $\begin{array}{lcc}\circ & \prime & \prime \prime \\
12 & 05 & 02.39 \\
76 & 15 & 16.71\end{array}$ & $\begin{array}{ccc}\circ & \prime & \prime \prime \\
192 & 03 & 23.36 \\
256 & 08 & 58.80\end{array}$ & \begin{tabular}{|} 
Log. meters. \\
4.2274765 \\
4.1413164
\end{tabular} & $\begin{array}{r}\text { Miles. } \\
10.491 \\
8.603\end{array}$ \\
\hline
\end{tabular}

PROSPECT, SHASTA COUNTY, CALIF. (Not occupled.)

Station mark: A prominent tree, probably trimmed, on summit of Prospect Peak, a well-known point northeast of Lassen.

[Latitude $40^{\circ} 34^{\prime} 24.741^{\prime \prime}$. Longitude $121^{\circ} 20^{\prime} 42.462^{\prime \prime}$.]

\begin{tabular}{|c|c|c|c|c|}
\hline To station- & Azimuth. & Back azimuth. & \multicolumn{2}{|c|}{ Distance. } \\
\hline 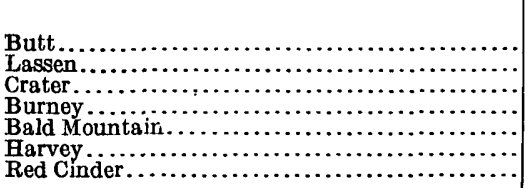 & \begin{tabular}{rcc}
\multicolumn{1}{c}{} & \multicolumn{1}{c}{$\prime \prime$} \\
4 & 57 & 40.42 \\
55 & 04 & 28.17 \\
121 & 03 & 23.72 \\
137 & 27 & 24.31 \\
175 & 41 & 59.44 \\
234 & 34 & 21.96 \\
315 & 37 & 33.99
\end{tabular} & \begin{tabular}{rcc}
\multicolumn{1}{c}{$\bullet$} & \multicolumn{1}{c}{ " } \\
184 & 56 & 06.51 \\
234 & 58 & 15.90 \\
300 & 52 & 43.35 \\
317 & 16 & 21.80 \\
355 & 40 & 43.18 \\
54 & 46 & 30.22 \\
135 & 41 & 25.35
\end{tabular} & \begin{tabular}{r|} 
Log. meters. \\
4.5979369 \\
4.2162828 \\
4.4305236 \\
4.5468666 \\
4.5620381 \\
4.5076492 \\
4.0787072
\end{tabular} & $\begin{array}{r}\text { Miles. } \\
24.620 \\
10.224 \\
16.745 \\
21.889 \\
22.667 \\
19.999 \\
7.448\end{array}$ \\
\hline
\end{tabular}

RED CINDER, IASSEN COUNTY, CALIF.

Station is on highest point of Red Cinder Butte, a well-known bald mountain, about 7 miles west-southwest of Stevens' summer cattle range on Pine Creek, and about 5 miles west of Silver Lake. The closest point of importance is Westwood, which is about 35 miles southeast. There is a fair road from Clover Valley and one from Bogards to the Stevens place. Wagons can not be taken beyond the Stevens place 
very well, though there is a very rough road from there to Silver Lake and another rough road from Clover Valley to Silver Lake. Animals are easily ridden to the point from almost any direction.

Signal: A cairn 7 feet high with 12-foot fir tree in center.

Station mark: A triangulation tablet set in solid rock under center of cairn.

[Latitude $40^{\circ} 29^{\prime} 46.810^{\prime \prime}$. Longitude $121^{\circ} 14^{\prime} 46.478^{\prime \prime}$.]

\begin{tabular}{|c|c|c|c|c|}
\hline To station- & Azimuth. & Back azimuth. & Distan & \\
\hline 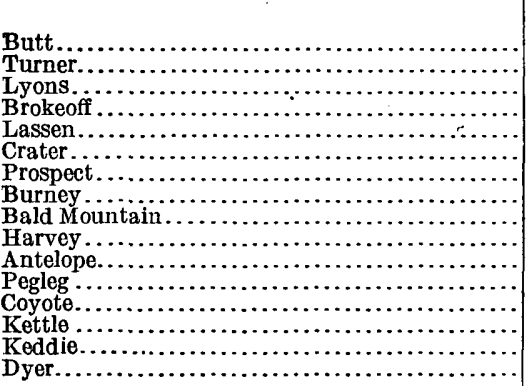 & \begin{tabular}{rcc}
\multicolumn{1}{c}{} & \multicolumn{1}{c}{$\prime \prime$} \\
20 & 58 & 36.28 \\
55 & 15 & 31.71 \\
57 & 11 & 16.16 \\
78 & 12 & 04.99 \\
87 & 49 & 59.07 \\
125 & 35 & 36.45 \\
135 & 31 & 25.35 \\
137 & 03 & 22.62 \\
166 & 10 & 10.87 \\
213 & 18 & 18.28 \\
249 & 19 & 01.21 \\
291 & 27 & 15.94 \\
302 & 52 & 38.30 \\
310 & 44 & 18.39 \\
322 & 38 & 38.02 \\
327 & 23 & 13.26
\end{tabular} & \begin{tabular}{rcc}
\multicolumn{1}{c}{} & $\prime$ & $\prime \prime$ \\
200 & 53 & 11.92 \\
235 & 01 & 08.76 \\
236 & 55 & 59.39 \\
257 & 59 & 54.67 \\
267 & 39 & 55.94 \\
305 & 21 & 04.94 \\
315 & 37 & 33.99 \\
316 & 48 & 28.73 \\
346 & 05 & 02.54 \\
33 & 26 & 34.22 \\
69 & 32 & 09.01 \\
111 & 38 & 43.97 \\
123 & 08 & 45.80 \\
131 & 04 & 45.22 \\
142 & 49 & 26.65 \\
147 & 31 & 32.92
\end{tabular} & $\begin{array}{c}\text { Log. meters. } \\
4.5196298 \\
4.5828454 \\
4.5993128 \\
4.4327868 \\
4.3402539 \\
4.5873406 \\
4.0787072 \\
4.6740212 \\
4.6655698 \\
4.5125597 \\
4.4837507 \\
4.4292118 \\
4.6231468 \\
4.7725326 \\
4.5914659 \\
4.5293560\end{array}$ & $\begin{array}{l}\text { Miles. } \\
\text { 20.558 } \\
23.779 \\
24.698 \\
16.832 \\
13.602 \\
24.027 \\
7.448 \\
29.334 \\
28.769 \\
20.226 \\
18.928 \\
16.694 \\
26.091 \\
35.803 \\
24.255 \\
\mathbf{2 1 . 0 2 1}\end{array}$ \\
\hline
\end{tabular}

ROUND, SHASTA COUNTY, CALIF.

A station of the U. S. Coast and Geodetic Survey, on the highest part of what is known as Round Mountain, just north of the post office of the same name.

Station mark: A $\frac{3}{5}$-inch copper bolt 3 inches long cemented into a drill hole in a large boulder which projects about 6 inches out of the ground, and directly above the bolt in the same drill hole is cemented an old-type station mark. A cross in the top of the copper bolt and another in the polished center of the disk mark the station.

Reference marks: A drill or punch hole in the top of a $\frac{3}{8}$-inch copper bolt, 3 inches long, which is leaded or cemented into a drill hole in a rock with the top of the bolt flush with the surface. Two such marks are at the following distances and azimuths from the station; 28.72 meters, $345^{\circ} 30^{\prime}$; and 6.43 meters $57^{\circ} 13^{\prime}$. The south reference mark is in the most eastern rock of a prominent group of rocks near the south end of the summit.

[Latitude $40^{\circ} 48^{\prime} 19.882^{\prime \prime}$. Longitude $121^{\circ} 57^{\prime} 26.873^{\prime \prime}$.]

\begin{tabular}{|c|c|c|c|c|}
\hline To station- & Azimuth. & Back azimuth. & Dista & nce. \\
\hline 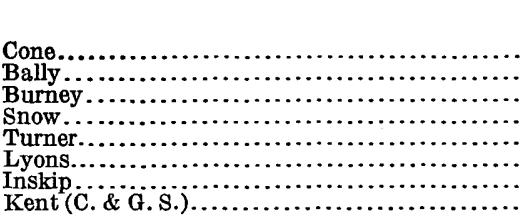 & $\begin{array}{rcc}\circ & \prime & \prime \prime \\
5 & 12 & 34.94 \\
69 & 14 & 22.18 \\
269 & 34 & 27.81 \\
296 & 01 & 23.38 \\
332 & 40 & 01.23 \\
334 & 10 & 02.03 \\
358 & 34 & 43.87 \\
35 & 40 & 46.89\end{array}$ & $\begin{array}{rcc}\circ & \prime \prime & \prime \prime \\
185 & 10 & 57.36 \\
248 & 47 & 16.01 \\
89 & 47 & 24.34 \\
116 & 08 & 00.37 \\
152 & 53 & 20.18 \\
154 & 22 & 27.03 \\
178 & 35 & 19.06 \\
215 & 10 & 27.70\end{array}$ & \begin{tabular}{r|} 
Log. meters. \\
4. 5898083 \\
4.7972333 \\
4.4448735 \\
4.2006062 \\
4.8007758 \\
4.7931190 \\
4.7116944 \\
5.0578276
\end{tabular} & $\begin{array}{r}\text { Miles. } \\
24.163 \\
38.957 \\
17.307 \\
9.862 \\
39.276 \\
38.590 \\
31.992\end{array}$ \\
\hline
\end{tabular}

SADDLE, SHASTA COUNTY, CALIF.

Station is on highest point of a bald mountain, locally known as Saddle Mountain, about 4 miles northwest of Fall River Mills. There is a good road from Fall River Mills to foot of mountain, thence north up ridge about a mile to summit. Animals can be ridden to summit.

Signal: A lone juniper tree 7 feet high on highest point.

Station mark: A bronze triangulation tablet set in rock 1.90 meters east of tree. Position of tablet computed. 
[Latitude $41^{\circ} 01^{\prime} 12.173^{\prime \prime}$. Longitude $121^{\circ} 29^{\prime} 43.938^{\prime \prime}$.]

\begin{tabular}{|c|c|c|c|c|}
\hline To station- & Azimuth. & Back azimuth. & Dista1 & \\
\hline 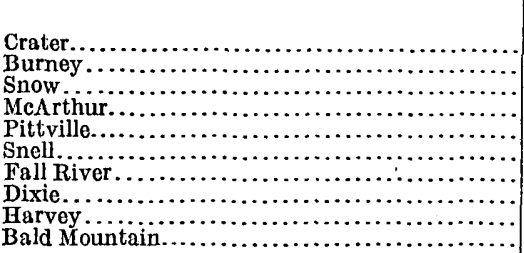 & \begin{tabular}{rcc}
\multicolumn{1}{c}{} &, & $\prime \prime$ \\
16 & 11 & 47.41 \\
25 & 11 & 06.23 \\
38 & 49 & 35.96 \\
250 & 22 & 26.00 \\
256 & 08 & 58.80 \\
281 & 14 & 02.60 \\
287 & 01 & 14.98 \\
288 & 24 & 32.07 \\
308 & 24 & 50.49 \\
323 & 01 & 35.05
\end{tabular} & \begin{tabular}{rcc}
\multicolumn{1}{c}{} & $\prime$ & $\prime \prime$ \\
196 & 06 & 58.37 \\
205 & 05 & 55.34 \\
218 & 38 & 05.41 \\
70 & 26 & 16.09 \\
76 & 15 & 16.71 \\
101 & 21 & 01.03 \\
107 & 03 & 33.63 \\
108 & 40 & 23.36 \\
128 & 42 & 56.44 \\
143 & 06 & 13.40
\end{tabular} & \begin{tabular}{r|} 
Log. meters. \\
4.5703853 \\
4. 4174010 \\
4.5964030 \\
3.9390518 \\
4.1413164 \\
4.1817569 \\
3.7129613 \\
4.5536808 \\
4.6962252 \\
4.2181368
\end{tabular} & $\begin{array}{r}\text { Miles. } \\
23.107 \\
16.246 \\
24.533 \\
5.400 \\
8.603 \\
9.443 \\
3.209 \\
22.235 \\
30.873 \\
10.268\end{array}$ \\
\hline
\end{tabular}

SNELL, LASSEN COUNTY, CALIF. (Not occupled. No check.)

Station mark: Center of high water tank at Mr. Snell's ranch about 10 miles east of Fall River Mills.

[Latitude $40^{\circ} 59^{\prime} 35.708^{\prime \prime}$. Longitude $121^{\circ} 19^{\prime} 06.227^{\prime \prime}$.]

\begin{tabular}{|c|c|c|c|c|}
\hline To station- & Azimuth. & Back azimuth. & Distar & \\
\hline 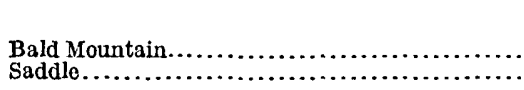 & $\begin{array}{rcc}0 & \prime & \prime \prime \\
25 & 59 & 21.91 \\
101 & 21 & 01.03\end{array}$ & $\begin{array}{ccc}\circ & , & \prime \prime \\
205 & 57 & 02.26 \\
281 & 14 & 02.60\end{array}$ & $\begin{array}{r}\text { Log. meters. } \\
4.0562255 \\
4.1817569\end{array}$ & $\begin{array}{r}\text { Miles. } \\
7.073 \\
9.443\end{array}$ \\
\hline
\end{tabular}

SNOW, SHASTA COUNTY, CALIF.

Station is on the more southern of two very prominent mountains about 7 or 8 miles west of Tamarack Flat, a well-known place about 14 miles south of Burney post office. The peak is locally known as Snow Mountain. The north point is a little higher than the point used but is covered with timber.

Animals can be ridden from Tamarack Flat, the summer home of Mr. Dan Covey, to summit of peak, but there is no well-defined trail.

Signal: A cairn 6 feet at base and 6 feet high with tree in center.

Station mark: A bronze triangulation tablet set in solid rock at center of cairn.

[Latitude $40^{\circ} 44^{\prime} 33.701^{\prime \prime}$. Longitude $121^{\circ} 47^{\prime} 19.900^{\prime \prime}$.]

\begin{tabular}{|c|c|c|c|c|}
\hline To station- & Azimuth. & Back azimuth. & Dista & ee. \\
\hline 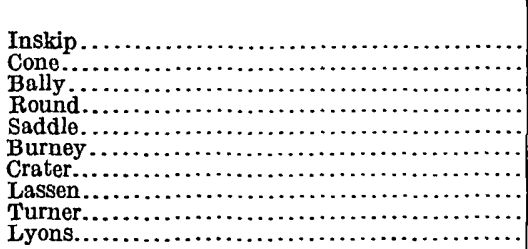 & $\begin{array}{rcc}\circ & \prime & \prime \prime \\
16 & 22 & 28.27 \\
29 & 21 . & 58.77 \\
78 & 17 & 06.06 \\
116 & 08 & 00.3 \prime \\
218 & 38 & 05.41 \\
242 & 17 & 41.34 \\
288 & 48 & 30.52 \\
319 & 26 & 55.34 \\
343 & 24 & 21.68 \\
345 & 27 & 06.72\end{array}$ & \begin{tabular}{rcc}
\multicolumn{1}{c}{$\circ$} & $\prime$ & $\prime \prime$ \\
196 & 16 & 28.31 \\
209 & 13 & 45.66 \\
257 & 43 & 24.74 \\
296 & 01 & 23.38 \\
38 & 49 & 35.96 \\
62 & 24 & 00.38 \\
108 & 55 & 10.59 \\
139 & 38 & 01.75 \\
163 & 31 & 05.16 \\
165 & 32 & 56.28
\end{tabular} & $\begin{array}{r}\text { Log. meters. } \\
4.6661722 \\
4.5611916 \\
4.8719614 \\
4.2006062 \\
4.5964030 \\
4.1865539 \\
4.1821344 \\
4.5691893 \\
4.7105858 \\
4.7039865\end{array}$ & $\begin{array}{r}\text { Miles. } \\
28.809 \\
22.623 \\
46.271 \\
9.862 \\
24.533 \\
9.548 \\
9.451 \\
23.043 \\
31.911 \\
31.429\end{array}$ \\
\hline
\end{tabular}

TURNER, TEHAMA COUNTY, CALIF.

Station is on the highest point of Turner Mountain, a prominent ridge about 5 miles south of Mineral post office. The U. S. Forest Service has a 9 by 9 foot cabin for lookout on the mountain, and there is a good trail from Mineral to the station.

Station mark: Center of U. S. Forest Service cabin.

Reference mark: A bronze triangulation tablet set in solid rock 1.7 feet east of northeast corner of cabin. Distance from station to reference mark, 2.60 meters; true azimuth from station to reference mark $232^{\circ} 55^{\prime}$. Position of center of cabin computed. 
[Latitude $40^{\circ} 17^{\prime} 57.638^{\prime \prime}$. Longitude $121^{\circ} 36^{\prime} 58.000^{\prime \prime}$.]

\begin{tabular}{|c|c|c|c|c|}
\hline To station- & Azimuth. & Back azimuth. & Dist & nce. \\
\hline 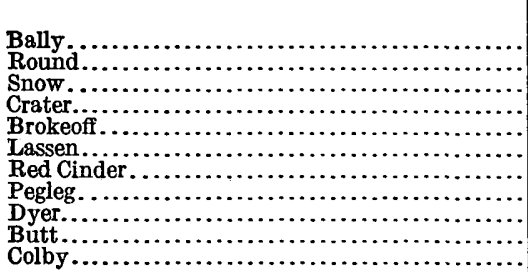 & $\begin{array}{ccc}\circ & \prime & \prime \prime \\
111 & 22 & 55.57 \\
152 & 53 & 20.18 \\
163 & 31 & 05.16 \\
179 & 45 & 54.30 \\
196 & 37 & 12.01 \\
204 & 17 & 03.66 \\
235 & 01 & 08.76 \\
257 & 46 & 39.74 \\
277 & 25 & 52.63 \\
294 & 37 & 24.56 \\
334 & 52 & 02.53\end{array}$ & \begin{tabular}{rcc}
\multicolumn{1}{c}{} & $\prime$ & $\prime \prime$ \\
290 & 42 & 40.44 \\
332 & 40 & 01.23 \\
343 & 24 & 21.68 \\
359 & 45 & 49.27 \\
16 & 39 & 25.66 \\
24 & 21 & 24.63 \\
55 & 15 & 31.71 \\
78 & 12 & 28.55 \\
97 & 48 & 31.95 \\
114 & 46 & 21.34 \\
154 & 55 & 35.78
\end{tabular} & $\begin{array}{r}\text { Log. meters. } \\
\text { 4. } 9730552 \\
\text { 4. } 8007758 \\
\text { 4. } 7105858 \\
\text { 4. } 6465358 \\
\text { 4. } 2304507 \\
\text { 4. } 3628774 \\
\text { 4. } 5828454 \\
\text { 4. } 7612451 \\
\text { 4. } 7001031 \\
\text { 4. } 3345578 \\
\text { 4. } 2648901\end{array}$ & $\begin{array}{r}\text { Miles. } \\
58.399 \\
39.276 \\
31.911 \\
27.535 \\
10.563 \\
14.329 \\
23.779 \\
35.859 \\
31.150 \\
13.425 \\
11.435\end{array}$ \\
\hline
\end{tabular}

WEST PROSPECT, SHASTA COUNTY, CALIF. (Not occupied.)

Highest point of West Prospect Peak, about 3 miles northwest of Prospect Peak.

[Latitude $40^{\circ} 35^{\prime} 39.336^{\prime \prime}$. Longitude $121^{\circ} 22^{\prime} 43.528^{\prime \prime}$.]

\begin{tabular}{|c|c|c|c|c|}
\hline To station- & Azimuth. & Back azimuth. & Dista1 & \\
\hline 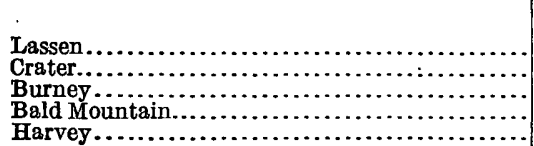 & $\begin{array}{rcc}\bullet & \prime & \prime \prime \\
42 & 13 & 10.68 \\
119 & 47 & 57.35 \\
138 & 24 & 57.70 \\
180 & 09 & 56.36 \\
240 & 37 & 04.07\end{array}$ & \begin{tabular}{rcc}
\multicolumn{1}{c}{} & $\prime$ & $\prime \prime$ \\
222 & 08 & 17.02 \\
299 & 38 & 35.70 \\
318 & 15 & 13.99 \\
0 & 09 & 59.10 \\
60 & 50 & 31.38
\end{tabular} & $\begin{aligned} \text { Log. meters. } \\
4.1995763 \\
4.3678633 \\
4.4998303 \\
4.5324311 \\
4.5231887\end{aligned}$ & $\begin{array}{r}\text { Miles. } \\
9.838 \\
14.495 \\
19.642 \\
21.173 \\
20.727\end{array}$ \\
\hline
\end{tabular}

Adelaida, Bonanza, Bradley, Bryson, Cayucos, Gonzales, Hollister, Jamesburg, Junipero Serra, King City, Paso Robles, Point Sur, San Benito, San Miguel, and San Simeon $15^{\prime}$ quadrangles.

Academy, Berendo, Biola Bliss Ranch, Bonita Ranch, Chaney Ranch, Chowchilla, Clovis, Dos Palos, Daulton, Elgin, Firebaugh, Fresno, Friant, Gregg, Gustine, Herndon, Indian Gulch, Ingomar, Jamesan, Jesbel, Kearney Park, Kentucky Well, Kerman, Kismet, Lanes Bridge, Le Grand, Lingard, Little Panoche, Iittle Table Mountain, Los Banos, Madera, Malaga, Mendota, Merced, No. 19, No. 21, No. 25, Orangedale, Oxalis, Plainsberg, Planada, Pozo Farm, Reedley, Round Mountain, Salt Slough, Sandy Mush, Sanger, San Joaquin, Santa Rita Ranch, Selma, Sheep Ranch, Squaw Valley, Sultana, Tranquillity, Trimmer, Tufts Ranch, Turner Ranch, and Wahtoke $7 \frac{{ }^{\prime}}{2}$ quadrangles.

FRESNO, MADERA, MERCED, MONTEREY, SAN BENITO, AND SAN LUIS OBISPO COUNTIES.

During the seasons of 1915,1916 , and 1917, C. F. Urquhart and L. F. Biggs extended triangulation over the above-named quadrangles.

Positions are on North American datum, derived from stations of the U. S. Geological Survey and the U. S. Coast and Geodetic Survey previously located in this area.

\section{ADOBE, MADERA COUNTY, CALIF., FROM SOUTH.}

On a small bald hill owned by the Krohn estate, situated on the south side of the Adobe ranch. The hill is about 1 mile north of Mr. Wootten's ranch, which is 12 miles from Madera on the Madera and O'Neals road.

Signal: A cairn 5 feet high.

Station mark: A triangulation tablet set in rock under center of cairn. 
[Latitude $37^{\circ} 02^{\prime} 03.094^{\prime \prime}$ (from south) $37^{\circ} 02^{\prime} 03.146^{\prime \prime}$ (from north), Longitude $119^{\circ} 51^{\prime} 41.132^{\prime \prime}$ (from south), $119^{\circ} 51^{\prime} 40.843^{\prime \prime}$ (from north).]

\begin{tabular}{|c|c|c|c|c|}
\hline To station- & Azimuth. & Back azimuth. & Distan & \\
\hline 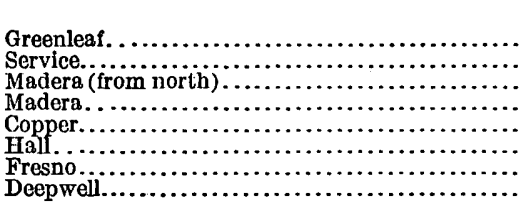 & $\begin{array}{rcc}\bullet & \prime & \prime \prime \\
21 & 23 & 22.93 \\
35 & 32 & 41.76 \\
65 & 13 & 35.53 \\
65 & 13 & 33.55 \\
300 & 18 & 15.16 \\
310 & 43 & 46.88 \\
348 & 45 & 23.68 \\
356 & 24 & 16.08\end{array}$ & $\begin{array}{ccc}\bullet & \prime & \prime \prime \\
201 & 20 & 41.41 \\
215 & 30 & 32.59 \\
245 & 06 & 23.10 \\
245 & 06 & 21.16 \\
120 & 26 & 02.75 \\
130 & 47 & 43.96 \\
168 & 48 & 02.27 \\
176 & 24 & 35.75\end{array}$ & $\begin{array}{r}\text { Log. meters. } \\
4.2610799 \\
\text { 3. } 9606108 \\
4.2917831 \\
4.2917561 \\
4.3479198 \\
4.1093124 \\
4.5266352 \\
4.1106596\end{array}$ & $\begin{array}{r}\text { Miles. } \\
11.335 \\
5.675 \\
12.166 \\
12.165 \\
13.844 \\
7.992 \\
20.892 \\
8.017\end{array}$ \\
\hline
\end{tabular}

ADOBE, MADERA COUNTY, CAIIF., FROM NORTH.

On a small bald hill belonging to the Krohn estate, situated on the south side of the Adobe ranch. The hill is about 1 mile north of Mr. Wootten's ranch, which is 12 miles from Madera and on the Madera and O'Neals road.

Signal and station mark: Cairn 5 feet high and triangulation tablet set in rock under its center.

[Latitude $37^{\circ} 02^{\prime} 03.146^{\prime \prime}$ (from north), $37^{\circ} 02^{\prime} 03.094^{\prime \prime}$ (from south). Longitude $119^{\circ} 51^{\prime} 40.843^{\prime \prime}$ (from north) $119^{\circ} 51^{\prime} 41.132^{\prime \prime}$ (from south).]

\begin{tabular}{|c|c|c|c|c|}
\hline To station- & Azimuth. & Back azimuth. & Dista & \\
\hline 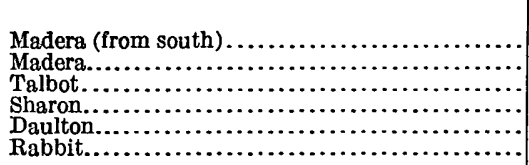 & $\begin{array}{rcc}\circ & \prime & \prime \prime \\
65 & 13 & 33.55 \\
65 & 13 & 35.53 \\
90 & 15 & 09.30 \\
107 & 06 & 30.82 \\
135 & 13 & 48.96 \\
151 & 35 & 52.69\end{array}$ & $\begin{array}{ccc}\circ & , & \prime \prime \\
245 & 06 & 21.16 \\
245 & 06 & 23.10 \\
270 & 09 & 07.42 \\
286 & 56 & 46.08 \\
315 & 09 & 25.36 \\
231 & 33 & 22.24\end{array}$ & $\begin{array}{r}\text { Log. meters. } \\
4.2917561 \\
4.2917831 \\
4.1717116 \\
4.3990592 \\
4.1853123 \\
4.1121673\end{array}$ & $\begin{array}{r}\text { Miles. } \\
12.165 \\
12.166 \\
9.227 \\
15.574 \\
9.521 \\
8.045\end{array}$ \\
\hline
\end{tabular}

ALFARATA, MERCED COUNTY, OALIF. (Not occupled.)

Station mark: Center of large silo at Alfarata, a siding on the Southern Pacific, R. R. about 3 miles southeast of Merced.

[Latitude $37^{\circ} 16^{\prime} 37.494^{\prime \prime}$. Longitude $120^{\circ} 26^{\prime} 18.745^{\prime \prime}$.]

\begin{tabular}{|c|c|c|c|c|}
\hline To station- & Azimuth. & Back azimuth. & Dist & nce. \\
\hline 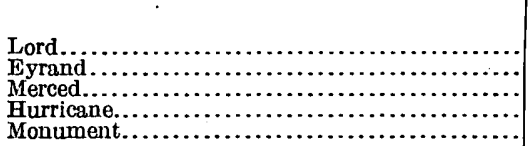 & $\begin{array}{rcc}- & \prime & \prime \prime \\
3 & 59 & 47.44 \\
61 & 49 & 13.55 \\
129 & 25 & 41.83 \\
226 & 59 & 15.20 \\
248 & 45 & 33.64\end{array}$ & \begin{tabular}{rcc}
\multicolumn{1}{c}{} & $\prime$ & $\prime \prime$ \\
183 & 59 & 40.10 \\
241 & 45 & 12.13 \\
309 & 24 & 02.88 \\
47 & 03 & 04.64 \\
68 & 47 & 44.74
\end{tabular} & \begin{tabular}{r|} 
Log. meters. \\
4. 0057353 \\
4. 0650404 \\
3. 7165755 \\
4. 1051387 \\
3. 7573088
\end{tabular} & $\begin{array}{r}\text { Miles. } \\
6.296 \\
7.218 \\
\text { 3. } 235 \\
\text { 7. } 916 \\
\text { 3. } 554\end{array}$ \\
\hline
\end{tabular}

ALISO MILL, MADERA COUNTY, CALIF., FROM SOUTH.

A lone wiadmill on the Miller \& Lux Aliso ranch about 12 miles east of Firebaugh. A wooden tank stands east of the mill, which is about 5 miles east of the Columbia ranch buildings.

Signal: The windmill pump rod.

Station mark: Center of well under mill. 
[Latitude $36^{\circ} 51^{\prime} 42.035^{\prime \prime}$ (irom south), $36^{\circ} 51^{\prime} 42.074^{\prime \prime}$ (from west). Longitude $120^{\circ} 15^{\prime} 21.156^{\prime \prime}$ (from south), $120^{\circ} 15^{\prime} 21.189^{\prime \prime}$ (from west).]

\begin{tabular}{|c|c|c|c|c|}
\hline To station- & Azimuth. & Back azimuth. & Distan & \\
\hline 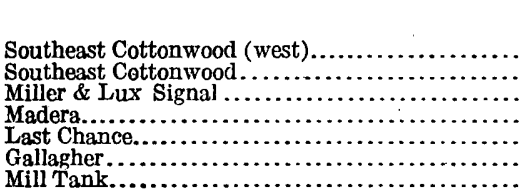 & $\begin{array}{ccc}\bullet & \prime & \prime \prime \\
182 & 04 & 39.08 \\
182 & 04 & 57.06 \\
223 & 33 & 53.69 \\
237 & 46 & 21.25 \\
269 & 48 & 35.19 \\
295 & 10 & 59.87 \\
317 & 52 & 05.65\end{array}$ & $\begin{array}{rcc}\bullet & \prime & \prime \prime \\
2 & 04 & 46.63 \\
2 & 05 & 04.62 \\
43 & 36 & 59.63 \\
57 & 53 & 22.54 \\
89 & 52 & 13.64 \\
115 & 16 & 03.59 \\
137 & 54 & 34.75\end{array}$ & \begin{tabular}{|r|} 
Log. meters. \\
3.9336920 \\
3.9335728 \\
4.0461435 \\
1.3120667 \\
3.9552171 \\
4.1422972 \\
3.9633800
\end{tabular} & $\begin{array}{r}\text { Miles. } \\
5.334 \\
5.332 \\
6.910 \\
12.747 \\
5.605 \\
8.623 \\
5.711\end{array}$ \\
\hline
\end{tabular}

ALISO, MADERA COUNTY, CALIF.

On a sand dune, 50 feet south of Madera-Firebaugh road, 8 miles east of Firebaugh, 3 miles east of Miller \& Lux ranch house, 0.1 mile east of jog in road at canal crossing.

Signal: Lumber.

Station mark: Iron post.

[Latitude $36^{\circ} 52^{\prime} 26.01^{\prime \prime}$. Longitude $120^{\circ} 18^{\prime} 54.90^{\prime \prime}$.]

\begin{tabular}{|c|c|c|c|c|}
\hline To station- & Azimuth. & Back azimuth. & Distan & \\
\hline 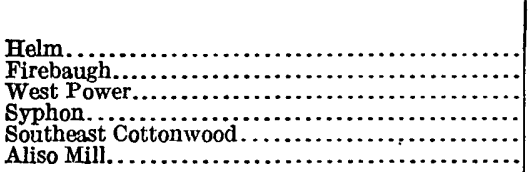 & $\begin{array}{ccc}\circ & , & \prime \prime \\
33 & 12 & 47.70 \\
-83 & 08 & 23.29 \\
114 & 34 & 34.41 \\
159 & 24 & 45.87 \\
217 & 46 & 26.93 \\
284 & 20 & 01.44\end{array}$ & $\begin{array}{rcc}\circ & \prime & \prime \prime \\
213 & 10 & 34.66 \\
263 & 03 & 16.23 \\
294 & 29 & 16.98 \\
339 & 22 & 42.22 \\
37 & 48 & 42.82 \\
104 & 22 & 09.68\end{array}$ & $\begin{array}{r}\text { Log. } \text { meters. } \\
4.0018767 \\
4.1061723 \\
4.1579846 \\
4.1604948 \\
3.9610265 \\
3.7375098\end{array}$ & $\begin{array}{l}\text { Miles. } \\
\quad 6.241 \\
7.935 \\
8.940 \\
8.992 \\
5.680 \\
3.395\end{array}$ \\
\hline
\end{tabular}

ALISO MILI, MADERA COUNTY, CALIF., FROM WEST.

A lone windmill on the Miller \& Lux Aliso ranch, about 12 miles east of Firebaugh. A wooden tank stands east of the mill, which is about 5 miles east of the Columbia ranch buildings.

Signal: The windmill pump rod.

Station mark: Center of well under mill.

[Latitude $36^{\circ} 51^{\prime} 42.074^{\prime \prime}$ (from west), $36^{\circ} 51^{\prime} 42.035^{\prime \prime}$ (from south). Longitude $120^{\circ} 15^{\prime} 21.189^{\prime \prime}$ (from west), $120^{\circ} 15^{\prime} 21.156^{\prime \prime}$ (from south).

\begin{tabular}{|c|c|c|c|c|}
\hline To station- & Azimuth. & Back azimuth. & Dista & \\
\hline 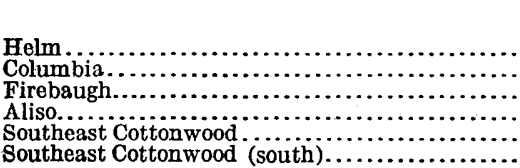 & $\begin{array}{rcc}\circ & \prime & \prime \prime \\
56 & 53 & 19.31 \\
80 & 05 & 51.98 \\
89 & 29 & 07.79 \\
104 & 22 & 09.66 \\
182 & 04 & 39.08 \\
182 & 04 & 57.06\end{array}$ & $\begin{array}{rcc}\circ & \prime & \prime \prime \\
236 & 48 & 58.18 \\
260 & 02 & 06.82 \\
269 & 21 & 52.57 \\
284 & 20 & 01.44 \\
2 & 04 & 46.63 \\
2 & 05 & 04.62\end{array}$ & $\begin{array}{r}\text { Log. meters. } \\
\text { 4. } 1103638 \\
3.9671745 \\
4.2546146 \\
3.7375098 \\
3.9336920 \\
3.9335728\end{array}$ & $\begin{array}{r}\text { Miles. } \\
8.012 \\
5.761 \\
11.168 \\
3.395 \\
5.334 \\
5.332\end{array}$ \\
\hline
\end{tabular}

ARMSTRONG, SAN BENITO COUNTY, CALIF.

The highest and most eastern of three hills on the summit of ridge forming the divide between Salinas and San Benito rivers. It is probably in San Benito County but very near the Monterey County line. There is an old road (traveled very little) between Mulberry post office and Gonzales, which passes just under hill on the north and west sides. By this road station is 7 or 8 miles from Mulberry. It is a good hcrseback route but very poor for buggy or wagon. The land on which station is situated is owned by the Armstrong brothers, of Salinas.

Signal: An oak tree in center of cairn 4 or 5 feet high.

Station mark: A triangulation tablet set in rock 1.03 meters from signal, azimuth from tablet to signal being $184^{\circ}$. 
[Latitude $36^{\circ} 36^{\prime} 40.597^{\prime \prime}$. Longitude $121^{\circ} 18^{\prime} 50.350^{\prime \prime}$.]

\begin{tabular}{|c|c|c|c|c|}
\hline To station- & Azimuth. & Back azimuth. & Distar & nce. \\
\hline 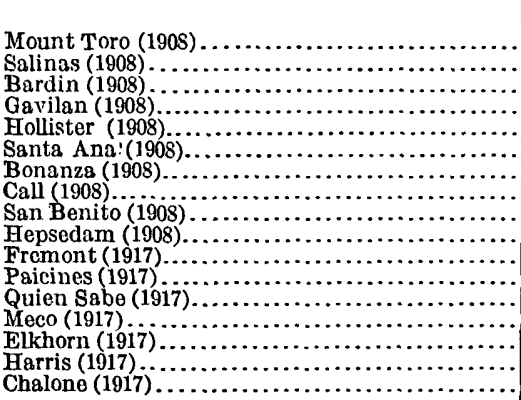 & $\begin{array}{ccc}\circ & \prime & \prime \prime \\
70 & 26 & 20.87 \\
102 & 50 & 34.18 \\
109 & 06 & 51.06 \\
131 & 08 & 43.66 \\
163 & 23 & 38.15 \\
192 & 30 & 48.53 \\
233 & 57 & 14.52 \\
270 & 19 & 47.40 \\
293 & 50 & 35.86 \\
306 & 40 & 45.35 \\
133 & 50 & 39.40 \\
182 & 28 & 40.26 \\
199 & 31 & 34.03 \\
235 & 32 & 41.24 \\
239 & 22 & 13.21 \\
308 & 03 & 41.89 \\
329 & 28 & 57.24\end{array}$ & \begin{tabular}{rcc}
\multicolumn{1}{c}{} & $\prime$ & $\prime \prime$ \\
250 & 15 & 48.18 \\
282 & 38 & 15.35 \\
288 & 59 & 29.20 \\
311 & 01 & 20.99 \\
343 & 20 & 25.50 \\
12 & 33 & 43.64 \\
54 & 09 & 12.15 \\
90 & 28 & 45.29 \\
114 & 14 & 30.21 \\
126 & 58 & 13.71 \\
313 & 43 & 52.00 \\
2 & 28 & 56.92 \\
19 & 34 & 59.63 \\
55 & 41 & 53.53 \\
59 & 28 & 21.12 \\
128 & 07 & 01.67 \\
149 & 33 & 12.32
\end{tabular} & $\begin{array}{r}\text { Log. meters. } \\
\text { 4. } 4477396 \\
4.4987001 \\
4.2891121 \\
4.3875319 \\
4.4459584 \\
4.5241889 \\
4.5659355 \\
4.3505389 \\
4.8177979 \\
4.7393296 \\
4.3702053 \\
4.2040293 \\
4.4064630 \\
4.4442413 \\
4.2499493 \\
4.0248336 \\
4.3225499\end{array}$ & 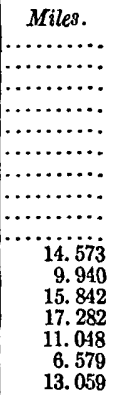 \\
\hline
\end{tabular}

ASH, MADERA COUNTY, CALIF., FROM WEST.

On the south bank of Ash Slough 7 miles southwest of Chowchilla. Follow the Robertson Boulevard for 2 miles to a signboard that reads "Alfalfa Colony," thence go westerly for 4 miles to Ash Slough, thence down south bank of slough to station.

Signal: A lumber quadripod with cross targets.

Station mark: Bench-mark post set under center of signal.

[Latitude $37^{\circ} 04^{\prime} 40.892^{\prime \prime}$ (from west), $37^{\circ} 04^{\prime} 40.685^{\prime \prime}$ (from north). Longitude $120^{\circ} 22^{\prime} 44.000^{\prime \prime}$ (from west), $120^{\circ} 22^{\prime} 43.820^{\prime \prime}$ (from north).

\begin{tabular}{|c|c|c|c|c|}
\hline To station- & Azimuth. & Back azimuth. & Dist & nee. \\
\hline 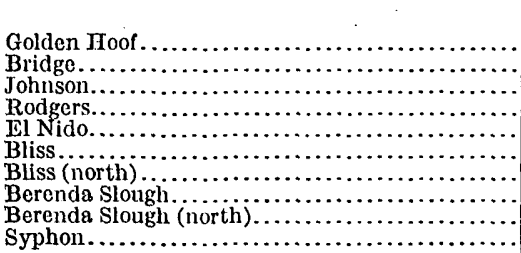 & \begin{tabular}{rcc}
\multicolumn{1}{c}{} & $\prime$ & $\prime \prime$ \\
38 & 28 & 15.43 \\
80 & 41 & 12.25 \\
82 & 48 & 09.25 \\
113 & 37 & 15.08 \\
122 & 11 & 44.83 \\
153 & 11 & 10.60 \\
153 & 10 & 57.57 \\
324 & 42 & 16.33 \\
324 & 42 & 03.31 \\
356 & 22 & 42.59
\end{tabular} & $\begin{array}{ccc}\circ & \prime & \prime \prime \\
218 & 23 & 50.80 \\
260 & 35 & 04.09 \\
262 & 43 & 16.03 \\
293 & 32 & 03.76 \\
302 & 07 & 41.59 \\
333 & 10 & 12.31 \\
333 & 09 & 59.28 \\
144 & 44 & 10.12 \\
144 & 43 & 57.11 \\
176 & 22 & 56.63\end{array}$ & \begin{tabular}{r|} 
Log. meters. \\
4. 2425475 \\
4. 1844950 \\
4. 0832402 \\
4. 1431363 \\
4. 0704060 \\
3. 7233285 \\
3. 7233285 \\
3. 9071271 \\
3. 9074271 \\
3. 9603124
\end{tabular} & $\begin{array}{r}\text { Miles. } \\
10.862 \\
9.503 \\
7.526 \\
8.639 \\
7.307 \\
3.286 \\
3.286 \\
5.021 \\
5.021 \\
5.671\end{array}$ \\
\hline
\end{tabular}

ASH, MADERA COUNTY, CALIF., FROM NORTH.

On the south bank of Ash Slough 7 miles southwest of Chowchilla. Follow the Robertson Boulevard for 2 miles to a signboard that reads "Alfalfa Colony," thence go westerly for 4 miles to Ash Slough, thence down south bank of slough to station.

Signal: A lumber quadripod with cross targets.

Station mark: Bench-mark post set under center of signal.

[Latitude $37^{\circ} 04^{\prime} 40.685^{\prime \prime}$ (from north), $37^{\circ} 04^{\prime} 40.892^{\prime \prime}$ (from west). Longitude $120^{\circ} 22^{\prime} 43.820^{\prime \prime}$ (from north), $120^{\circ} 22^{\prime} 44.000^{\prime \prime}$ (from west).]

\begin{tabular}{|c|c|c|c|c|}
\hline Tostation- & Azimuth. & Back azimuth. & Dista: & \\
\hline 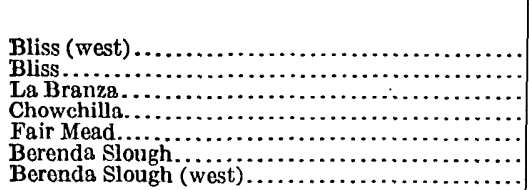 & $\begin{array}{ccc}\circ & \prime & \prime \prime \\
153 & 11 & 10.60 \\
153 & 10 & 57.57 \\
205 & 04 & 07.55 \\
242 & 55 & 47.35 \\
270 & 09 & 33.15 \\
324 & 42 & 03.31 \\
324 & 42 & 16.33\end{array}$ & \begin{tabular}{rcc}
\multicolumn{1}{c}{ - } & $\prime \prime$ \\
\hdashline 333 & 09 & 59.28 \\
25 & 06 & 12.32 \\
63 & 00 & 03.56 \\
90 & 16 & 18.75 \\
144 & 43 & 57.11 \\
144 & 44 & 10.12
\end{tabular} & $\begin{aligned} \text { Log. meters. } \\
\text { 3. } 7223285 \\
\text { 3. } 7233285 \\
4.0804456 \\
4.0709244 \\
4.2205790 \\
3.9074271 \\
\text { 3. } 9074271\end{aligned}$ & $\begin{array}{r}\text { Miles. } \\
3.286 \\
3.286 \\
7.478 \\
7.316 \\
10.326 \\
\mathbf{5 . 0 2 1} \\
\mathbf{5 . 0 2 1}\end{array}$ \\
\hline
\end{tabular}




\section{BAREILlas, MERCED COUNTY, CALIF.}

On a bald peak, the lowest and most eastern of three peaks on land owned by J. C. Bareillas. About 3 miles a little east of south from the San Luis ranch, which is on the road between Los Banos and Hollister, about 15 miles from the former. The signal can be seen from this ranch.

Signal and station mark: A cairn 7 feet high.

Reference mark: A tablet set in rock, 1.72 meters south of the signal.

[Latitude $37^{\circ} 01^{\prime} 33.559^{\prime \prime}(1908-15), 37^{\circ} 01^{\prime} 33.570^{\prime \prime}$ (1912-13). Longitude $121^{\circ} 02^{\prime} 19.450^{\prime \prime}$ (1908-15), 121 $02^{\prime} 19.456^{\prime \prime}(1912-13)$.]

\begin{tabular}{|c|c|c|c|c|}
\hline To station- & Azimuth. & Back azimuth. & Distar & nce. \\
\hline 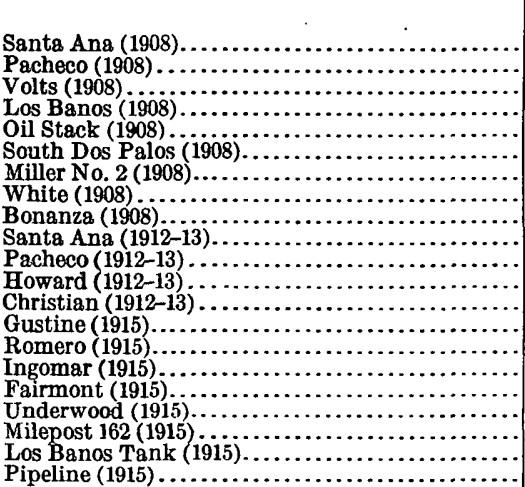 & 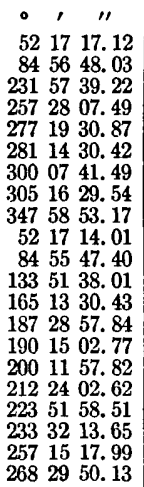 & \begin{tabular}{rcc}
\multicolumn{1}{c}{} & $\prime$ & $\prime \prime$ \\
232 & 10 & 17.21 \\
264 & 47 & 49.87 \\
52 & 01 & 38.54 \\
77 & 34 & 57.41 \\
97 & 31 & 32.73 \\
101 & 28 & 22.03 \\
120 & 21 & 51.29 \\
125 & 23 & 37.82 \\
168 & 00 & 59.10 \\
232 & 10 & 14.11 \\
264 & 46 & 49.16 \\
313 & 45 & 06.23 \\
345 & 11 & 30.55 \\
7 & 30 & 19.34 \\
10 & 15 & 53.96 \\
20 & 14 & 31.54 \\
32 & 29 & 59.59 \\
43 & 59 & 12.88 \\
53 & 38 & 41.10 \\
77 & 22 & 17.66 \\
88 & 33 & 11.41
\end{tabular} & $\begin{array}{r}\text { Log. meters. } \\
4.3394971 \\
4.3460746 \\
4.0952256 \\
4.2360987 \\
4.4756665 \\
4.5422771 \\
4.6076025 \\
4.3343198 \\
4.3972398 \\
4.3394995 \\
4.3461620 \\
4.3468888 \\
4.2836434 \\
4.4073108 \\
4.0710566 \\
4.2601790 \\
4.4346644 \\
4.4086518 \\
4.2349810 \\
4.2466538 \\
3.9172138\end{array}$ & 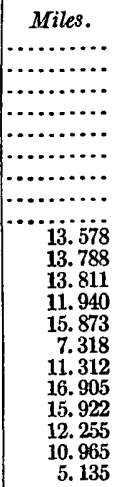 \\
\hline
\end{tabular}

BEAR HILL, FRESNO COUNTY, CALIF.

On the west end of Bear Hill about 18 miles east of Sanger.

Signal: A 20-foot trimmed live-oak tree.

Reference mark: A tablet set in rock, azimuth from tablet to signal, $61^{\circ} 30^{\prime}$; distance, 19 feet.

Position of signal tree computed.

[Latitude $36^{\circ} 44^{\prime} 45.570^{\prime \prime}$. Tongitude $119^{\circ} 16^{\prime} 57.336^{\prime \prime}$.]

\begin{tabular}{|c|c|c|c|c|}
\hline To station- & Azimuth. & Back azimuth. & Distar & \\
\hline 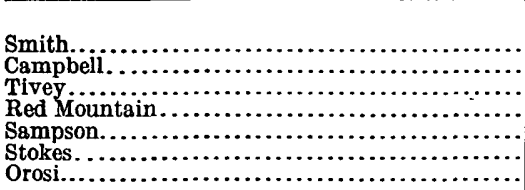 & \begin{tabular}{rcc}
\multicolumn{1}{c}{} & $\prime$ & $\prime \prime$ \\
17 & 09 & 44.36 \\
65 & 48 & 48.26 \\
88 & 19 & 03.89 \\
141 & 24 & 49.96 \\
260 & 48 & 49.85 \\
343 & 14 & 54.32 \\
354 & 38 & 55.52
\end{tabular} & \begin{tabular}{rcc}
\multicolumn{1}{c}{} & $\prime$ & $\prime \prime$ \\
197 & 07 & 29.62 \\
245 & 43 & 50.25 \\
268 & 15 & 01.33 \\
321 & 20 & 38.75 \\
80 & 55 & 19.29 \\
163 & 17 & 56.84 \\
174 & 39 & 44.62
\end{tabular} & $\begin{array}{r}\text { Log. meters. } \\
4.2790135 \\
4.1323649 \\
4.0027310 \\
4.2215142 \\
4.2134869 \\
4.4217145 \\
4.3411858\end{array}$ & $\begin{aligned} \text { Miles. } \\
11.813 \\
8.428 \\
6.253 \\
10.348 \\
10.159 \\
16.408 \\
13.631\end{aligned}$ \\
\hline
\end{tabular}

BELMONT, MERCED COUNTY, CALIF.

The center of west one of two large concrete silos at the Miller \& Lux Belmon $t$ ranch, about 4 miles north of the town of Dos Palos.

Signal and station mark: Center of silo.

[Latitude $37^{\circ} 02^{\prime} 14.498^{\prime \prime}$. Longitude $120^{\circ} 37^{\prime} 23.560^{\prime \prime}$.]

\begin{tabular}{|c|c|c|c|c|}
\hline To station- & Azimuth. & Back azimuth. & Dis & nce. \\
\hline 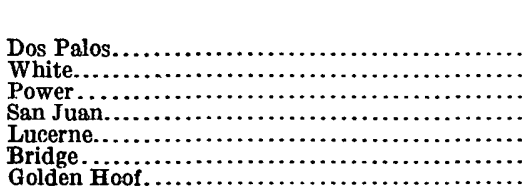 & \begin{tabular}{rcc}
\multicolumn{1}{c}{} & $\prime$ & $\prime \prime$ \\
1 & 54 & 00.79 \\
54 & 42 & 58.16 \\
103 & 13 & 15.90 \\
148 & 19 & 47.67 \\
205 & 20 & 22.17 \\
253 & 02 & 13.05 \\
310 & 07 & 04.05
\end{tabular} & \begin{tabular}{rcc}
\multicolumn{1}{c}{} & $\prime$ & $\prime \prime$ \\
181 & 53 & 56.30 \\
234 & 35 & 06.76 \\
283 & 09 & 48.67 \\
329 & 18 & 35.13 \\
25 & 21 & 26.25 \\
73 & 04 & 54.97 \\
130 & 11 & 28.83
\end{tabular} & $\begin{array}{r}\text { Log. meters. } \\
3.7452289 \\
4.3759685 \\
3.9410284 \\
3.7529990 \\
3.7878512 \\
3.8415535 \\
4.1533735\end{array}$ & $\begin{array}{r}\text { Miles. } \\
3.456 \\
14.768 \\
5.425 \\
3.518 \\
3.812 \\
4.314 \\
8.845\end{array}$ \\
\hline
\end{tabular}


BERENDÁ SCEOOL, MADERA COUNTY, CALIF. (Not occupied.)

Cupola on schoolhouse at Berenda, a small town on the Southern Pacific R. R., between Merced and Madera.

[Latitude $37^{\circ} 02^{\prime} 16.200^{\prime \prime}$. Longitude $120^{\circ} 09^{\prime} 09.998^{\prime \prime}$.]

\begin{tabular}{|c|c|c|c|c|}
\hline To station- & Azimuth. & Back azimuth. & Dista & \\
\hline 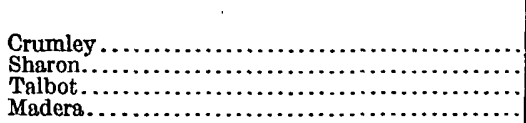 & $\begin{array}{rcc}\bullet & \prime & \prime \prime \\
28 & 56 & 32.28 \\
195 & 42 & 53.00 \\
271 & 46 & 17.76 \\
316 & 30 & 38.35\end{array}$ & \begin{tabular}{rcc}
\multicolumn{1}{c}{} & $\prime$ & $\prime \prime$ \\
208 & 56 & 01.75 \\
15 & 43 & 40.65 \\
91 & 50 & 47.80 \\
136 & 33 & 57.29
\end{tabular} & $\begin{array}{l}\text { Log. meters. } \\
\text { 3. } 4133365 \\
3.8577427 \\
4.0447607 \\
4.0749278\end{array}$ & $\begin{array}{r}\text { Miles. } \\
1.609 \\
4.478 \\
.6 .888 \\
7.384\end{array}$ \\
\hline
\end{tabular}

BERENDA SLOUGH, MADERA COUNTY, CALIF., FROM WEST.

On the northwest end of bridge over Berenda Slough 9 miles southwest of Chowchilla. Follow Robertson Boulevard to Fillmore Avenue, thence go east to EIm Avenue, thence south to station.

Signal: Lumber structure with cross targets and flag.

Reference mark: Second wall post 8 feet from northwest corner of bridge. [Latitude $37^{\circ} 01^{\prime} 06.9 ، 2^{\prime \prime}$ (from west), $37^{\circ} 01^{\prime} 06.714^{\prime \prime}$ (from north). Longitude $120^{\circ} 19^{\prime} 35.134^{\prime \prime}$ (from west),
$120^{\circ} 19^{\prime} 34.939^{\prime \prime}$ (from north.]

\begin{tabular}{|c|c|c|c|c|}
\hline 'To station- & Azimuth. & Back azimuth. & Dis & nce. \\
\hline 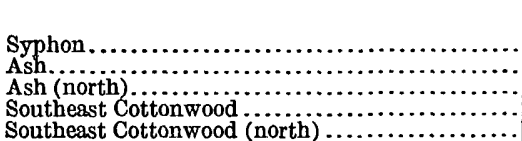 & $\begin{array}{rcc}\circ & \prime & \prime \prime \\
58 & 28 & 24.56 \\
144 & 44 & 10.12 \\
144 & 43 & 57.11 \\
323 & 14 & 44.48 \\
323 & 14 & 31.48\end{array}$ & $\begin{array}{ccc}\circ & \prime & \prime \prime \\
238 & 26 & 44.91 \\
324 & 42 & 16.33 \\
324 & 42 & 03.31 \\
143 & 17 & 24.80 \\
143 & 17 & 30.32\end{array}$ & \begin{tabular}{|c|} 
Log. meters. \\
3. 6814664 \\
3.9074271 \\
3.9074271 \\
4.0422536 \\
4.0422536
\end{tabular} & $\begin{array}{r}\text { Miles. } \\
2.984 \\
5.021 \\
5.021 \\
6.849 \\
6.849\end{array}$ \\
\hline
\end{tabular}

BERENDA SLOUGH, MADERA COUNTY, CALIF., FROM NORTH.

On the northwest end of bridge over Berenda Slough 9 miles southwest of Chowchilla. Follow Robertson Boulevard to Fillmore Avenue, thence go east to Elm Avenue, thence south to station.

Signal: Lumber structure with cross targets and flag.

Reference mark: Second wall post 8 feet from northwest corner of bridge. [Latitule $37^{\circ} 01^{\prime} 06.714^{\prime \prime}$ (from north), $37^{\circ} 01^{\prime} 06.912^{\prime \prime}$ (from west). Longitude $120^{\circ} 19^{\prime} 34.939^{\prime \prime}$ (from north),
$120^{\circ} 19^{\prime} 35.134^{\prime \prime}$ (from west.]

\begin{tabular}{|c|c|c|c|c|}
\hline To station- & Aximuth. & Back azimuth. & Dista & ce. \\
\hline 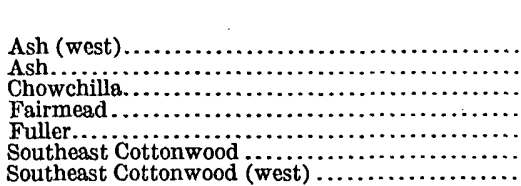 & $\begin{array}{ccc}\circ & \prime & \prime \prime \\
144 & 44 & 10.12 \\
114 & 43 & 57.11 \\
205 & 58 & 27.83 \\
241 & 18 & 24.28 \\
284 & 10 & 05.20 \\
323 & 14 & 31.48 \\
323 & 14 & 44.48\end{array}$ & \begin{tabular}{rcc}
\multicolumn{1}{c}{} & \multicolumn{1}{c}{$\prime \prime$} \\
324 & 42 & 16.33 \\
324 & 42 & 03.31 \\
26 & 00 & 49.99 \\
61 & 23 & 15.80 \\
104 & 13 & 09.51 \\
143 & 17 & 30.32 \\
143 & 17 & 24.80
\end{tabular} & $\begin{array}{r}\text { Log. meters. } \\
3.9074271 \\
3.9074271 \\
4.1235994 \\
4.1343554 \\
3.8925604 \\
4.0422536 \\
4.0422536\end{array}$ & $\begin{array}{r}\text { Miles. } \\
5.021 \\
5.021 \\
8.259 \\
8.467 \\
4.852 \\
6.849 \\
6.849\end{array}$ \\
\hline
\end{tabular}

BIOLA, FRESNO COUNTY, CALIF.

Station and signal: Large water tank at Biola station, which is on a branch railroad, 7 miles northeast of Kerman.

Signal and station mark: Center of tank. 
[Latitude $36^{\circ} 48^{\prime} 02.139^{\prime \prime}$. Longitude $120^{\circ} 00^{\prime} 53.596^{\prime \prime}$.]

\begin{tabular}{|c|c|c|c|c|}
\hline To station- & Azimuth. & Back azimuth. & Distan & e. \\
\hline 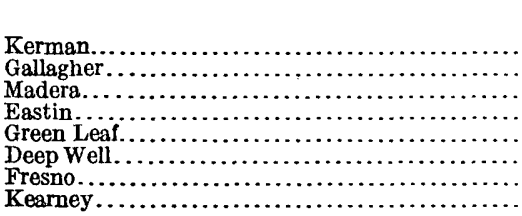 & $\begin{array}{rcc}\circ & , & \prime \prime \\
25 & 24 & 43.26 \\
95 & 32 & 50.98 \\
166 & 56 & 02.01 \\
183 & 57 & 22.10 \\
218 & 10 & 49.56 \\
227 & 57 & 47.61 \\
289 & 08 & 39.24 \\
315 & 08 & 42.51\end{array}$ & \begin{tabular}{rcc}
\multicolumn{1}{c}{} & $\prime$ & $\prime \prime$ \\
205 & 23 & 05.25 \\
275 & 29 & 14.74 \\
346 & 54 & 22.35 \\
3 & 57 & 34.34 \\
38 & 13 & 39.73 \\
48 & 03 & 30.59 \\
109 & 16 & 39.85 \\
135 & 12 & 10.77
\end{tabular} & $\begin{array}{c}\text { Log. meters. } \\
\text { 3.9763586 } \\
3.9537156 \\
4.2594110 \\
3.8643524 \\
4.0557672 \\
4.2900607 \\
4.3313870 \\
4.0878869\end{array}$ & $\begin{array}{r}\text { Miles. } \\
5.884 \\
5.586 \\
11.292 \\
4.547 \\
7.065 \\
12.117 \\
13.327 \\
7.607\end{array}$ \\
\hline
\end{tabular}

BISHOFF, MONTEREY COUNTY, CALIF.

On sharp peak 3 or 4 miles west-southwest of King City. It is the highest point in the foothills overlooking valley from west. Station can be reached from King City by going west and crossing the long bridge over Salinas River, thence through gate to Mr. Allan Dillon's residence, thence up canyon passing Fred Hansen's residence and on to head of canyon which is at foot of peak. From this point on foot or horseback to summit.

Signal: A greasewood bush about 11 feet high.

Station mark: A triangulation tablet set in top of rock at foot of signal.

[Latitude $36^{\circ} 11^{\prime} 25.380^{\prime \prime}$ (1909), $36^{\circ} 11^{\prime} 25.170^{\prime \prime}(1917)$. Longitude $121^{\circ} 12^{\prime} 00.610^{\prime \prime}(1909), 121^{\circ} 12^{\prime} 00.538^{\prime \prime}$ (1917).]

\begin{tabular}{|c|c|c|c|c|}
\hline To station- & Azimuth. & Back azimuth. & Distar & nce. \\
\hline 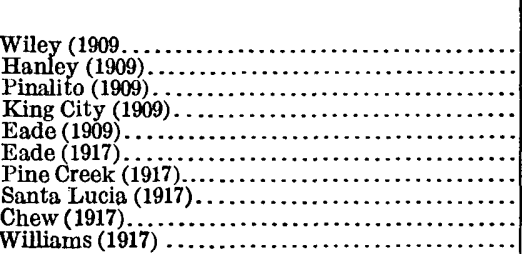 & \begin{tabular}{rcc}
\multicolumn{1}{c}{} & $\prime$ & $\prime \prime$ \\
141 & 44 & 49.87 \\
176 & 05 & 44.53 \\
218 & 15 & 59.06 \\
245 & 21 & 57.81 \\
259 & 17 & 50.12 \\
259 & 17 & 57.41 \\
14 & 28 & 01.55 \\
75 & 58 & 30.31 \\
112 & 13 & 02.44 \\
325 & 47 & 57.01
\end{tabular} & \begin{tabular}{rcc}
\multicolumn{1}{c}{} & $\prime$ & $\prime \prime$ \\
321 & 42 & 12.45 \\
356 & 05 & 10.34 \\
38 & 20 & 19.54 \\
65 & 24 & $\mathbf{3 5 . 5 4}$ \\
79 & 26 & 01.25 \\
79 & 26 & 08.61 \\
194 & 27 & 02.84 \\
255 & 50 & 46.80 \\
292 & 00 & 00.14 \\
145 & 54 & 59.25
\end{tabular} & \begin{tabular}{r|} 
Log. meters. \\
4.0309625 \\
4.3242187 \\
4.2490167 \\
3.8655621 \\
4.3249351 \\
4.3250001 \\
3.9985104 \\
4.3061705 \\
4.5520767 \\
4.5047955
\end{tabular} & 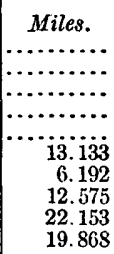 \\
\hline
\end{tabular}

\section{BLACKSMITH, MERCED COUNTY, CALIF.}

About 12 miles by road northeast of Merced, 2 miles northeast of Merced reservoir, 1 mile south of Lower Merced Falls road, on summit of hill in pasture.

Signal: Lumber structure with cross targets.

Station mark: Iron bench-mark post centered under signal.

Latitude 37 ' $24^{\prime} 26.708^{\prime \prime}$. Longitude $120^{\circ} 23^{\prime} 05.779 "$.]

\begin{tabular}{|c|c|c|c|c|}
\hline To station- & Azimuth. & Back azimuth. & Dista1 & \\
\hline 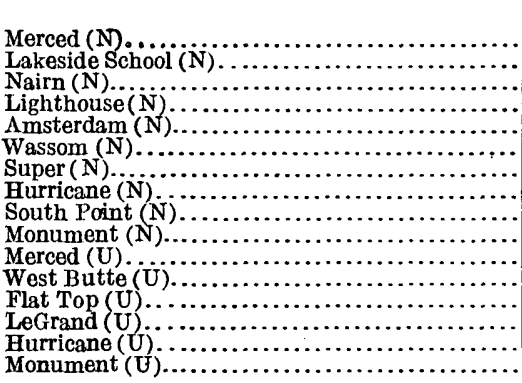 & $\begin{array}{rcc}\circ & \prime & \prime \prime \\
38 & 01 & 52.87 \\
38 & 26 & 17.53 \\
69 & 48 & 02.42 \\
96 & 18 & 00.51 \\
98 & 35 & 36.48 \\
118 & 09 & 29.67 \\
122 & 37 & 10.43 \\
321 & 23 & 46.90 \\
337 & 23 & 40.96 \\
357 & 06 & 20.74 \\
38 & 01 & 52.87 \\
263 & 40 & 02.21 \\
301 & 39 & 45.25 \\
320 & 23 & 49.51 \\
321 & 23 & 18.44 \\
357 & 06 & 17.29\end{array}$ & $\begin{array}{rcc}\circ & \prime & \prime \prime \\
217 & 58 & 17.90 \\
218 & 24 & 34.38 \\
249 & 43 & 11.34 \\
276 & 12 & 48.26 \\
278 & 29 & 30.80 \\
298 & 06 & 03.03 \\
302 & 35 & 38.27 \\
141 & 25 & 40.76 \\
157 & 25 & 55.17 \\
177 & 06 & 36.17 \\
217 & 58 & 17.90 \\
83 & 45 & 34.87 \\
121 & 49 & 44.89 \\
140 & 29 & 02.11 \\
141 & 25 & 12.29 \\
177 & 06 & 32.73\end{array}$ & \begin{tabular}{r|} 
Log. meters. \\
4.1511041 \\
3.8277024 \\
4.0992809 \\
4.1042753 \\
4.1750801 \\
3.9767853 \\
3.6461410 \\
3.8690886 \\
4.1515650 \\
4.0937836 \\
4.1511041 \\
4.1317959 \\
4.4566259 \\
4.2993034 \\
3.8689719 \\
4.0937954
\end{tabular} & $\begin{array}{r}\text { Miles. } \\
8.800 \\
4.179 \\
7.810 \\
7.900 \\
9.299 \\
5.890 \\
2.751 \\
4.597 \\
8.809 \\
7.711 \\
8.799 \\
8.417 \\
17.782 \\
12.378 \\
4.595 \\
7.712\end{array}$ \\
\hline
\end{tabular}

$\mathrm{N}=$ Work done by C. L. Nelson in 1914; $\mathrm{U}=$ work done by C. F. Urquhart in 1914. 


\section{BLISS, MERCED COUNTY, CALIF., FROM WEST.}

In Mr. Bliss's pasture field, 0.75 mile northeast of the ranch house. To reach it go westerly from Minturn, a station on the Southern Pacific R. R., past the Sieca Vista ranch. The signal is about 7 miles from Minturn.

Signal: Quadripod with cross targets.

Station mark: Iron bench-mark post centered under signal.

[Latitude $37^{\circ} 07^{\prime} 13.776^{\prime \prime}$ (from north), $37^{\circ} 07^{\prime} 13.989^{\prime \prime}$ (from west). Longitude $120^{\circ} 24^{\prime} 20.465$ (from north), $120^{\circ} 24^{\prime} 20.632^{\prime \prime}$ ([rom west).]

\begin{tabular}{|c|c|c|c|c|}
\hline To station- & Azimuth. & Back azimuth. & Dis & nce. \\
\hline 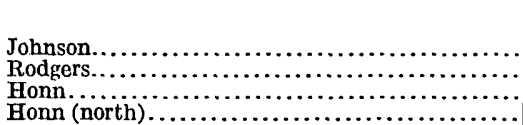 & $\begin{array}{rcc}\circ & \prime & \prime \prime \\
57 & 03 & 25.13 \\
94 & 41 & 02.16 \\
117 & 56 & 01.82 \\
117 & 55 & 48.79\end{array}$ & $\begin{array}{ccc}\circ & \prime & \prime \prime \\
236 & 59 & 30.05 \\
274 & 36 & 49.01 \\
\cdots & & \end{array}$ & $\begin{array}{c}\text { Log. meters. } \\
4.0597503 \\
4.0165461 \\
3.6941369 \\
3.6941369\end{array}$ & $\begin{array}{r}\text { Miles. } \\
.7 .130 \\
6.455 \\
3.072 \\
3.072\end{array}$ \\
\hline
\end{tabular}

BLISS, MERCED COUNTY, CALIF., FROM NORTH.

In Mr. Bliss's pasture field, 0.75 mile northeast of the ranch house. To reach it go westerly from Minturn, a station on the Southern Pacific R. R., past the Sieca Vista ranch. The signal is about 7 miles from Minturn.

Signal: Quadripod with cross targets.

Station mark: İron bench-mark post centered under signal.

[Latitude $37^{\circ} 07^{\prime} 13.776^{\prime \prime}$ (from north), $37^{\circ} 07^{\prime} 13.989^{\prime \prime}$ (from west). Longitude $12 \mathrm{c}^{\circ} 24^{\prime} 20.465^{\prime \prime}$ (from north) $120^{\circ} 24^{\prime} 20.032^{\prime \prime}$ (from west).]

\begin{tabular}{|c|c|c|c|c|}
\hline To station- & Aximuth. & Back azimuth. & Distar & \\
\hline 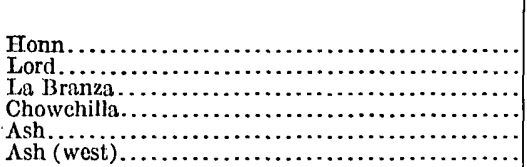 & $\begin{array}{ccc}\circ & , & \prime \prime \\
117 & 55 & 48.79 \\
153 & 49 & 00.49 \\
230 & 25 & 56.00 \\
267 & 08 & 37.56 \\
333 & 09 & 59.28 \\
333 & 10 & 12.31\end{array}$ & \begin{tabular}{rcc}
\multicolumn{1}{c}{} & $\prime$ & $\prime \prime$ \\
297 & 51 & 01.94 \\
333 & 47 & 32.95 \\
50 & 28 & 59.19 \\
87 & 13 & 52.22 \\
153 & 10 & 57.57 \\
153 & 11 & 10.60
\end{tabular} & $\begin{array}{c}\text { Log. meters. } \\
\text { 3. } 6941369 \\
\text { 3. } 9085808 \\
\text { 3. } 9871177 \\
\text { 4. } 1101135 \\
\text { 3. } 7233285 \\
\text { 3. } 7233285\end{array}$ & $\begin{array}{r}\text { Miles. } \\
\text { 3. } 072 \\
5.034 \\
6.032 \\
8.007 \\
3.286 \\
3.286\end{array}$ \\
\hline
\end{tabular}

BONANZA, MERCED COUNTY, CALIF.

On a high, bald peak, which has oak trees growing just below the summit on the north side. It is about 3 miles northeast of Bonanza post office. From Tres Pinos on the Southern Pacific R. R. there is a road to Bonanza post office, distance 24 miles, thence the way is up canyon 1 mile to Mr. Sepulveda's ranch. From this place there is a trail or wood road about a mile farther up canyon and trail from there to summit. Animals can be ridden all the way up. Station can also be reached from the San Joaquin side by going up a wood road to summit, thence south along ridge to highest point.

Signal: A cairn 8 feet high.

Station mark: A tablet in rock placed under center of signal.

[Latitude $36^{\circ} 48^{\prime} 21.558^{\prime \prime}$. Longitudo $120^{\circ} 58^{\prime} 49.786^{\prime \prime}$.]

\begin{tabular}{|c|c|c|c|c|}
\hline Tostation- & Azimuth. & Back azimuth. & Dista & e. \\
\hline 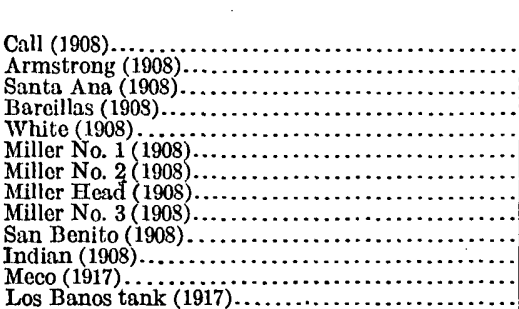 & $\begin{array}{rcc}\circ & , & \prime \prime \\
18 & 49 & 51.53 \\
51 & 09 & 12.15 \\
116 & 11 & 52.03 \\
168 & 00 & 59.10 \\
226 & 11 & 02.36 \\
2: 8 & 32 & 29.67 \\
262 & 15 & 19.98 \\
269 & 01 & 03.61 \\
278 & 06 & 28.35 \\
328 & 00 & 19.79 \\
308 & 48 & 18.31 \\
49 & 15 & 11.38 \\
203 & 02 & 01.39\end{array}$ & \begin{tabular}{rcc}
\multicolumn{1}{c}{} &, & $\prime \prime$ \\
198 & 46 & 53.03 \\
233 & 57 & 14.52 \\
296 & 02 & 47.44 \\
347 & 58 & 53.17 \\
46 & 16 & 03.78 \\
68 & 40 & 49.17 \\
82 & 27 & 21.95 \\
89 & 16 & 18.47 \\
98 & 21 & 35.12 \\
148 & 12 & 21.82 \\
128 & 57 & 25.73 \\
229 & 12 & 26.10 \\
23 & 06 & 54.01
\end{tabular} & $\begin{array}{r}\text { Log. meters. } \\
4.3615909 \\
4.5659355 \\
4.3988290 \\
4.3972398 \\
\text { 4. } 2361938 \\
\text { 4. } 3456991 \\
\text { 4. } 4788602 \\
\text { 4. } 5781093 \\
\text { 4. } 5791323 \\
\text { 4. } 7558232 \\
\text { 4. } 4651214 \\
\text { 3. } 9561009 \\
4.4878415\end{array}$ & $\begin{array}{r}\text { Miles. } \\
14.287 \\
22.871 \\
15.566 \\
15.509 \\
10.711 \\
13.774 \\
18.71 .6 \\
23.521 \\
23.577 \\
35.414 \\
18.133 \\
5.616 \\
19.107\end{array}$ \\
\hline
\end{tabular}


BORLAND, FRESNO COUNTY, CALIF.

On the Miller \& Lux Borland ranch about 7 miles south and a little east of Mendota.

Signal and station mark: Center of No. 5 smokestack.

[Latitude $36^{\circ} 40^{\prime} 43.086^{\prime \prime}$. Longitude $120^{\circ} 19^{\prime} 41.698^{\prime \prime}$.]

\begin{tabular}{|c|c|c|c|c|}
\hline Tostation- & Azimuth. & Back azimuth. & Distar & \\
\hline 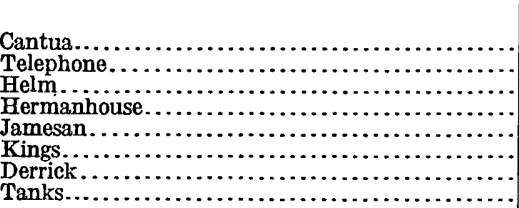 & $\begin{array}{rcc}\circ & \prime & \prime \prime \\
12 & 48 & 38.32 \\
95 & 07 & 34.13 \\
161 & 52 & 40.46 \\
188 & 30 & 04.18 \\
240 & 44 & 25.56 \\
266 & 18 & 52.71 \\
293 & 36 & 32.91 \\
344 & 36 & 06.12\end{array}$ & \begin{tabular}{rcc}
\multicolumn{1}{c}{} & $\prime$ & $\prime \prime$ \\
192 & 46 & 57.81 \\
275 & 00 & 53.67 \\
341 & 50 & 55.70 \\
8 & 30 & 31.01 \\
60 & 48 & 51.34 \\
86 & 22 & 30.47 \\
113 & 43 & 21.33 \\
164 & 36 & 40.68
\end{tabular} & $\begin{array}{r}\text { Log. meters. } \\
4.2769295 \\
4.2229194 \\
4.1447886 \\
3.8768482 \\
4.1019662 \\
3.9575854 \\
4.2685567 \\
3.7336756\end{array}$ & $\begin{array}{r}\text { Miles. } \\
11.757 \\
10.382 \\
8.672 \\
4.679 \\
7.858 \\
5.636 \\
11.532 \\
3.365\end{array}$ \\
\hline
\end{tabular}

BOULDER, MONTEREY COUNTY, CALIF. (Not occupied.)

This is probably the old U. $\dot{\mathrm{S}}$. Coast and Geodetic Survey station Mount Boulder.

[Latitude $36^{\circ} 23^{\prime} 10.206^{\prime \prime}$. Longitude $121^{\circ} 47^{\prime} 15.408^{\prime \prime}$.]

\begin{tabular}{|c|c|c|c|c|}
\hline Tostation- & Azimuth. & Back azimuth. & Dista1 & \\
\hline 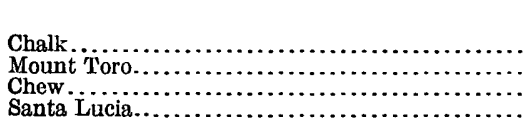 & $\begin{array}{ccc}\circ & \prime & \prime \prime \\
189 & 19 & 22.31 \\
225 & 47 & 20.74 \\
292 & 45 & 56.55 \\
308 & 39 & 54.41\end{array}$ & \begin{tabular}{rcc}
\multicolumn{1}{c}{} & $\prime$ & $\prime \prime$ \\
9 & 20 & 32.99 \\
45 & 53 & 42.90 \\
112 & 53 & 45.91 \\
128 & 53 & 00.84
\end{tabular} & \begin{tabular}{|r|} 
Log. meters. \\
4.2614170 \\
4.3487418 \\
4.3309168 \\
4.6290707
\end{tabular} & $\begin{aligned} \text { Miles. } \\
11.344 \\
13.871 \\
13.313 \\
26.450\end{aligned}$ \\
\hline
\end{tabular}

BRIDGE, MERCED COUNTY, CALIF. (Not occupied.)

A target on bridge over San Joaquin River, on road from Dos Palos to Merced.

[Latitude $37^{\circ} 03^{\prime} 20.171^{\prime \prime}$. Longitude $120^{\circ} 32^{\prime} 54.791^{\prime \prime}$.]

\begin{tabular}{|c|c|c|c|c|}
\hline Tostation- & Azimuth. & Back azimuth. & Distar & \\
\hline 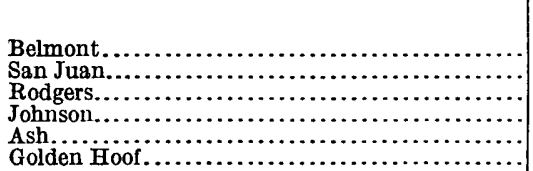 & $\begin{array}{rrr}\circ & \prime & \prime \prime \\
73 & 04 & 54.97 \\
106 & 14 & 51.03 \\
196 & 11 & 43.79 \\
252 & 36 & 03.07 \\
260 & 35 & 04.09 \\
339 & 17 & 04.66\end{array}$ & \begin{tabular}{rcc}
\multicolumn{1}{c}{} & $\prime$ & $\prime \prime$ \\
253 & 02 & 13.05 \\
286 & 11 & 31.84 \\
16 & 12 & 40.91 \\
72 & 37 & 18.02 \\
80 & 41 & 12.25 \\
159 & 18 & 47.73
\end{tabular} & \begin{tabular}{|l|} 
Log. meters. \\
3.8415535 \\
4.0005040 \\
3.9233892 \\
3.5078316 \\
4.1844950 \\
4.0783409
\end{tabular} & $\begin{array}{r}\text { Miles. } \\
4.314 \\
6.221 \\
5.209 \\
2.001 \\
9.503 \\
7.442\end{array}$ \\
\hline
\end{tabular}

BULLARD FLAGPOLE, FRESNO COUNTY, CALIF. (Not occupied.)

Station is the flagpole on tank house at the Bullard Club, about 8 miles north of Fresno.

[Latitude $36^{\circ} 50^{\prime} 55.335^{\prime \prime}$. Longitude $119^{\circ} 49^{\prime} 57.942^{\prime \prime}$.]

\begin{tabular}{|c|c|c|c|c|}
\hline To station- & Azimuth. & Back azimuth. & Distan & \\
\hline 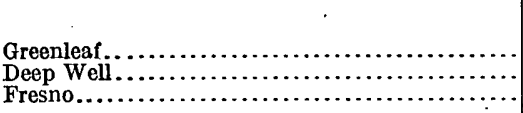 & $\begin{array}{ccc}\circ & \prime & \prime \prime \\
111 & 21 & 27.91 \\
167 & 14 & 35.42 \\
342 & 07 & 59.68\end{array}$ & $\begin{array}{ccc}\circ & , & \prime \prime \\
291 & 17 & 44.83 \\
347 & 13 & 5.3 .11 \\
162 & 09 & 36.12\end{array}$ & $\begin{array}{r}\text { Log. meters. } \\
\text { 3. } 9950910 \\
\text { 3. } 8977887 \\
\text { 4. } 1147057\end{array}$ & $\begin{array}{r}\text { Miles. } \\
6.144 \\
4.911 \\
8.092\end{array}$ \\
\hline
\end{tabular}


Call, saN BENITO COUNTT, CALIF.

On a brushy peak on ridge south of Tres Pinos Creek and east of San Benito River. Can be reached by leaving the Hollister and Idria road on grade between twenty-fifth and twenty-sixth mileposts (from former place) and going south up ridge about 5 or 6 miles. Shortly after leaving main road the Bradford quicksilver mine is passed, and farther up the ridge there are two deserted houses. Station is at the end of open live-oak country on summit of ridge; signal can be seen about 0.25 mile south on a brushy peak. Mr. Call once lived near the summit, and the ridge is sometimes known by his name.

Signal: A cairn 7 feet high on highest point of peak.

Station mark: A triangulation tablet set in large rock 1 meter from signal; azimuth, $318^{\circ}$.

[Latitude $36^{\circ} 36^{\prime} 35.463^{\prime \prime}$. Longitude $121^{\circ} 03^{\prime} 48.417^{\prime \prime}$.]

\begin{tabular}{|c|c|c|c|c|}
\hline To station- & Azimuth. & Back azimuth. & Distar & nec. \\
\hline 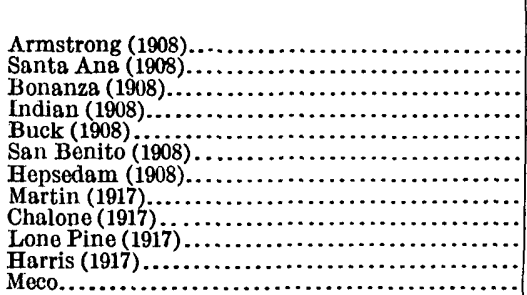 & \begin{tabular}{rcc}
\multicolumn{1}{c}{} & $\prime$ & $\prime \prime$ \\
90 & 28 & 45.29 \\
155 & 18 & 27.69 \\
198 & 46 & 53.03 \\
263 & 22 & 48.63 \\
292 & 45 & 03.85 \\
305 & 11 & 17.80 \\
326 & 40 & 15.64 \\
13 & 21 & 19.43 \\
33 & 18 & 14.47 \\
43 & 45 & 33.95 \\
65 & 42 & 21.44 \\
182 & 01 & 20.75
\end{tabular} & \begin{tabular}{rcc}
\multicolumn{1}{c}{} & $\prime$ & $\prime \prime$ \\
270 & 19 & 47.40 \\
335 & 12 & 23.05 \\
18 & 49 & 51.53 \\
83 & 34 & 52.92 \\
112 & 51 & 53.38 \\
125 & 26 & 15.75 \\
146 & 48 & 47.96 \\
193 & 20 & 01.54 \\
213 & 13 & 32.69 \\
223 & 41 & 49.00 \\
245 & 36 & 43.70 \\
2 & 01 & 34.25
\end{tabular} & \begin{tabular}{r|} 
Log. meters. \\
4. 3505389 \\
4. 5576284 \\
4. 3615909 \\
4. 4823341 \\
4. 2680060 \\
4. 6633038 \\
4. 5926168 \\
4. 1490672 \\
4. 3318580 \\
4. 1330026 \\
4.1893173 \\
4.2006530
\end{tabular} & 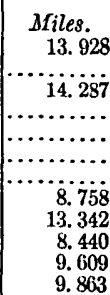 \\
\hline
\end{tabular}

CAMPBELL MOUNTAIN, FRESNO COUNTY, CALIF.

On the west end of Campbell Mountain about 8 miles east of Sanger.

Signal and station mark: Cairn 7 feet high with chiseled circle under center.

Reference mark: Bronze tablet 4 feet west of center of signal. Position of signal computed.

[Latitude $36^{\circ} 41^{\prime} 45.003^{\prime \prime}$. Longitude $119^{\circ} 25^{\prime} 15.748^{\prime \prime}$.]

\begin{tabular}{|c|c|c|c|c|}
\hline Tostation- & Azimuth. & Back azimuth. & Dista & \\
\hline 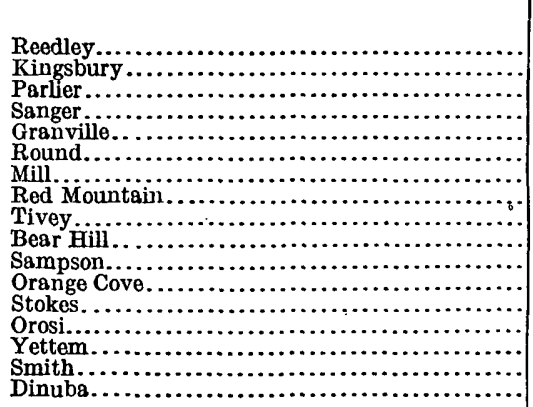 & \begin{tabular}{rcc|} 
& $\prime$ & $\prime \prime$ \\
14 & 06 & 46.96 \\
30 & 06 & 26.65 \\
45 & 19 & 52.90 \\
96 & 49 & 12.00 \\
107 & 03 & 13.71 \\
150 & 01 & 11.96 \\
152 & 30 & 06.56 \\
186 & 01 & 09.75 \\
203 & 39 & 20.67 \\
245 & 43 & 50.25 \\
253 & 55 & 59.90 \\
309 & 15 & 59.79 \\
314 & 33 & 00.41 \\
318 & 23 & 44.52 \\
326 & 33 & 54.39 \\
331 & 42 & 30.42 \\
349 & 31 & 40.94
\end{tabular} & \begin{tabular}{rcc|}
0 & $\prime$ & $\prime \prime$ \\
194 & 05 & 40.67 \\
210 & 01 & 47.80 \\
225 & 16 & 02.53 \\
276 & 44 & 21.13 \\
286 & 57 & 25.32 \\
329 & 58 & 04.92 \\
332 & 26 & 27.92 \\
6 & 01 & 57.10 \\
23 & 40 & 16.25 \\
65 & 48 & 48.26 \\
74 & 07 & 27.20 \\
129 & 19 & 56.83 \\
134 & 41 & 00.03 \\
138 & 29 & 30.91 \\
146 & 40 & 01.73 \\
151 & 45 & 13.20 \\
169 & 32 & 55.96
\end{tabular} & $\begin{array}{l}\text { Log. maters. } \\
\text { 4. } 0539363 \\
\text { 4. } 3654139 \\
\text { 4. } 1298555 \\
\text { 4. } 0851381 \\
\text { 4. } 1796546 \\
\text { 4. } 1987067 \\
4.2922609 \\
\text { 4. } 2713925 \\
3.7595096 \\
4.1323649 \\
\text { 4. } 4721482 \\
\text { 4. } 1052375 \\
4.4484236 \\
\text { 4. } 3374712 \\
\text { 4. } 446605 \\
\text { 4. } 1555507 \\
\text { 4. } 2357838\end{array}$ & $\begin{array}{r}\text { Miles. } \\
7.035 \\
14.413 \\
8.379 \\
7.559 \\
9.397 \\
9.819 \\
12.179 \\
11.608 \\
3.572 \\
8.428 \\
18.429 \\
7.917 \\
17.449 \\
13.515 \\
17.299 \\
8.890 \\
10.694\end{array}$ \\
\hline
\end{tabular}

CANTUA, FRESNO COUNTY, CAIIF.

On a slight raise about 4 miles southwest of the D. G. Longtin ranch house, where good water may be had. There is a cabin 0.25 mile northeast of signal.

Signal: A timber structure with one target 8 by 10 feet, facing northeast.

Station mark: Tablet in rock. 
[Latitude $36^{\circ} 30^{\prime} 44.517^{\prime \prime}$. Longitude $120^{\circ} 22^{\prime} 30.295^{\prime \prime}$.]

\begin{tabular}{|c|c|c|c|c|}
\hline To station- & Azimuth. & Back azimuth. & Dis & nce. \\
\hline 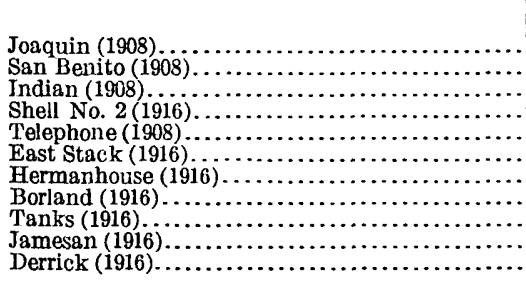 & \begin{tabular}{rcc}
\multicolumn{1}{c}{} & $\prime$ & $\prime \prime$ \\
19 & 21 & 35.75 \\
56 & 50 & 05.50 \\
114 & 31 & 04.13 \\
124 & 04 & 05.82 \\
148 & 00 & 14.53 \\
164 & 49 & 39.00 \\
191 & 33 & 22.06 \\
192 & 46 & 57.81 \\
203 & 02 & 22.53 \\
211 & 41 & 56.43 \\
242 & 30 & 52.34
\end{tabular} & $\begin{array}{rcc}\circ & \prime & \prime \prime \\
199 & 18 & 37.72 \\
236 & 40 & 30.42 \\
294 & 18 & 30.89 \\
303 & 58 & 57.54 \\
327 & 55 & 15.38 \\
344 & 48 & 34.08 \\
11 & 35 & 29.43 \\
12 & 48 & 38.32 \\
23 & 04 & 37.47 \\
31 & 48 & 02.27 \\
62 & 39 & 20.40\end{array}$ & $\begin{array}{r}\text { Log. meters. } \\
4.3534305 \\
4.4598650 \\
4.5379847 \\
4.1912724 \\
4.3712011 \\
4.0149715 \\
4.4221967 \\
4.2769295 \\
4.1576810 \\
4.4617664 \\
4.3782577\end{array}$ & $\begin{array}{r}\text { Miles. } \\
14.021 \\
17.915 \\
21.445 \\
9.652 \\
14.607 \\
6.432 \\
16.427 \\
11.757 \\
8.934 \\
17.994 \\
14.846\end{array}$ \\
\hline
\end{tabular}

CASTLE MOUNT, MONTEREY AND FRESNO COUNTIES, CALIF.

A station of the U. S. Coast and Geodetic Survey on a high hogback locally known as Black Mountain. It can be reached from Coalinga by rough road and trail past Parkfield, Turkey Flat, and Taylor's ranch, where accommodations can be had.

Station mark: A copper bolt in center of top of theodolite pier.

Reference marks: Copper nails (four) leaded in holes in rocks, approximately north, south, east, and west, as follows: North 4.525 feet; south 6.540 feet; east 3.520 feet; and west 10.390 feet. Concrete pier for meridian instrument was 1.6 meters due east of station, and wooden pier for vertical circle 10.642 meters southwest.

Note (by C. F. U. 1917).-The best way to reach the station is from Edward Taylor's ranch 6 miles east of Parkfield post office. Animals can be ridden to summit.

[Latitude $35^{\circ} 56^{\prime} 21.338^{\prime \prime}$. Longitude $120^{\circ} 20^{\prime} 22.908^{\prime \prime}$.]

\begin{tabular}{|c|c|c|c|c|}
\hline Tostation- & Azimuth. & Back azimuth. & Dista & cee. \\
\hline 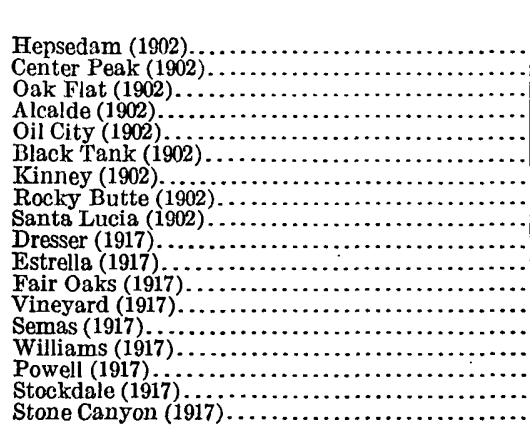 & \begin{tabular}{rcc}
\multicolumn{1}{c}{$c$} & $\prime$ & $\prime \prime$ \\
133 & 51 & 16.62 \\
\hdashline 137 & 07 & 51.82 \\
147 & 07 & 81.29 \\
164 & 49 & 51.29 \\
178 & 11 & 52.42 \\
181 & 45 & 18.82 \\
191 & 42 & 50.45 \\
65 & 10 & 20.33 \\
103 & 35 & 26.85 \\
28 & 02 & 45.92 \\
35 & 34 & 11.34 \\
51 & 31 & 30.06 \\
60 & 34 & 17.50 \\
82 & 40 & 09.16 \\
91 & 31 & 58.16 \\
101 & 43 & 14.13 \\
103 & 55 & 06.76 \\
116 & 23 & 51.90
\end{tabular} & \begin{tabular}{rcc}
\multicolumn{1}{c}{} & $\prime$ & $\prime \prime$ \\
313 & 34 & 08.71 \\
$\ldots 327$ & 01 & 07.45 \\
344 & 47 & 38.90 \\
358 & 11 & 24.69 \\
1 & 45 & 35.92 \\
11 & 45 & 05.29 \\
244 & 45 & 05.68 \\
282 & 57 & 22.08 \\
207 & 54 & 36.29 \\
215 & 27 & 50.43 \\
231 & 15 & 34.56 \\
240 & 22 & 33.77 \\
262 & 23 & 07.28 \\
271 & 08 & 40.76 \\
281 & 29 & 56.41 \\
283 & 46 & 36.87 \\
296 & 16 & 20.56
\end{tabular} & \begin{tabular}{|r|} 
Log. meters. \\
4.7804191 \\
4.5887373 \\
4.5001282 \\
4.3325900 \\
4.5722254 \\
4.3727723 \\
4.4500045 \\
4.8558353 \\
4.9994343 \\
4.6519052 \\
4.4488243 \\
4.7201779 \\
4.5395326 \\
4.6439980 \\
4.7758227 \\
4.5408143 \\
4.3503908 \\
4.3318977
\end{tabular} & 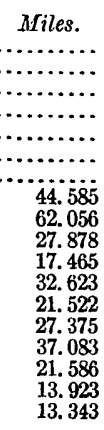 \\
\hline
\end{tabular}

CHALK, MONTEREY COUNTY, CALIF.

On a low ridge about 11 miles from Salinas. It can be reached from Salinas by taking the Monterey road to a point about 0.8 mile beyond summit, where there is a water trough and a signboard reading "To Del Monte ranch." Here take left hand road and continue on it to forks of three roads. Take the middle one and continue on it until just before Salinas Gun Club is reached. The signal can be seen from here, not more than half a mile distant.

Signal: A tree supported by three poles.

Station mark: A tablet set in rock. Azimuth from tablet to signal is $153^{\circ}$, distance, 15.67 meters. 
[Latitude $36^{\circ} 32^{\prime} 54.655^{\prime \prime}(1917), 36^{\circ} 32^{\prime} 54.674^{\prime \prime}$ (1908). Longitude $121^{\circ} 45^{\prime} 16.497^{\prime \prime}$ (1917), $121^{\circ} 45^{\prime} 16.506^{\prime \prime}$ (1908).]

\begin{tabular}{|c|c|c|c|c|}
\hline To station- & Azimuth. & Back azimuth. & Dista & uce. \\
\hline 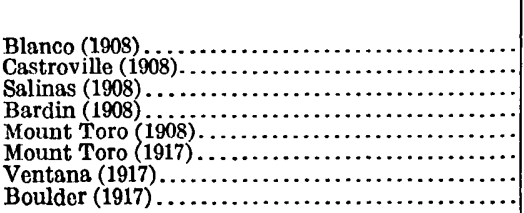 & \begin{tabular}{rcc}
\multicolumn{1}{c}{} & \multicolumn{1}{c}{$\prime \prime$} \\
173 & 03 & 08.46 \\
179 & 35 & 53.78 \\
211 & 50 & 41.93 \\
237 & 34 & 31.84 \\
280 & 39 & 22.41 \\
280 & 39 & 27.34 \\
346 & 42 & 44.72 \\
9 & 20 & 32.99
\end{tabular} & \begin{tabular}{rcc|}
\multicolumn{1}{c}{} & $\prime$ & $\prime \prime$ \\
353 & 02 & 35.22 \\
359 & 35 & 49.69 \\
31 & 54 & 09.60 \\
57 & 42 & 56.21 \\
100 & 44 & 34.51 \\
100 & 44 & 39.43 \\
166 & 45 & 29.80 \\
189 & 19 & 22.31
\end{tabular} & \begin{tabular}{r|} 
Log. meters. \\
4. 0587699 \\
4. 3347198 \\
4. 2145242 \\
4. 3958912 \\
4. 1228971 \\
4. 1228971 \\
4. 4782974 \\
4. 2614170
\end{tabular} & 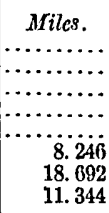 \\
\hline
\end{tabular}

ChaLoNe, moNTEREy aNd SAN BENITO CoUNTIES, CALIF.

- On the northwestern of the well-known Chalone Peaks, the highest peaks on east side of valley. There is a wagon road from Soledad to foot of peak, which is about 10 miles from Soledad. The peak is very near the line between Monterey and San Benito counties.

Signal: A cairn 7 feet high.

Station mark: A triangulation tablet in rock under center of cairn.

[Latitude $36^{\circ} 26^{\prime} 53.023^{\prime \prime}$ (1917), 36 $26^{\prime} 53.04^{\prime \prime}$ (1909). Longitude $121^{\circ} 11^{\prime} 41.821^{\prime \prime}$ (1917), $121^{\circ} 11^{\prime} 41.93^{\prime \prime}$ (1909).]

\begin{tabular}{|c|c|c|c|c|}
\hline Tostation- & Azimuth. & Back arimuth. & Distar & nce. \\
\hline 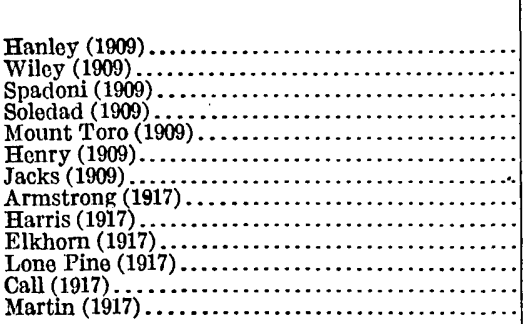 & \begin{tabular}{rcc}
\multicolumn{1}{c}{} & \multicolumn{1}{c}{$\prime \prime$} \\
14 & 15 & 40.93 \\
19 & 26 & 24.76 \\
74 & 09 & 52.49 \\
78 & 29 & 53.67 \\
103 & 17 & 48.75 \\
114 & 38 & 45.60 \\
125 & 21 & 35.71 \\
149 & 33 & 12.32 \\
168 & 40 & 01.22 \\
189 & 44 & 23.36 \\
196 & 19 & 38.67 \\
213 & 13 & 32.69 \\
243 & 32 & 55.08
\end{tabular} & \begin{tabular}{rcc}
\multicolumn{1}{c}{} & $\prime$ & $\prime \prime$ \\
194 & 14 & 55.55 \\
199 & 23 & 35.79 \\
254 & 02 & 13.78 \\
258 & 25 & 07.13 \\
283 & 03 & 02.53 \\
294 & 34 & 38.03 \\
305 & 13 & 12.83 \\
329 & 28 & 57.24 \\
348 & 39 & 05.71 \\
9 & 46 & 15.24 \\
16 & 20 & 35.64 \\
33 & 18 & 14.47 \\
63 & 36 & 18.71
\end{tabular} & $\begin{array}{r}\text { Log. meters. } \\
3.8884515 \\
4.3290724 \\
4.3012792 \\
4.0886495 \\
4.5809152 \\
4.0571365 \\
4.4110000 \\
4.3225499 \\
4.0722502 \\
4.4402035 \\
3.9283823 \\
4.3318580 \\
3.9788655\end{array}$ & 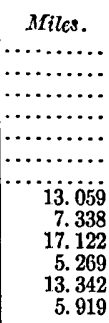 \\
\hline
\end{tabular}

CHEW, MONTEREY COUNTY, CALIF.

The highest point of Chews Ridge, 7 miles by road south of Jamesburg. It can be reached by following the Tassajara Spring road from Jamesburg 7 miles to the summit, then continue $\frac{1}{8}$ mile beyond summit to a trail with signboard marked "Lookout Station $\frac{1}{4}$ mile." Follow this trail to station, which is a U. S. Forest Service lookout station.

Signal and station mark: A pole with cross targets.

Reference mark: A tablet set on the line Chew-Boulder 10 feet from center of signa Azimuth from tablet to signal, $112^{\circ} 54^{\prime}$.

[Latitude $36^{\circ} 18^{\prime} 40.501^{\prime \prime}$. Iongitude $121^{\circ} 34^{\prime} 03.510^{\prime \prime}$.]

\begin{tabular}{|c|c|c|c|c|}
\hline To station- & Azimuth. & Back azimuth. & Distar & \\
\hline 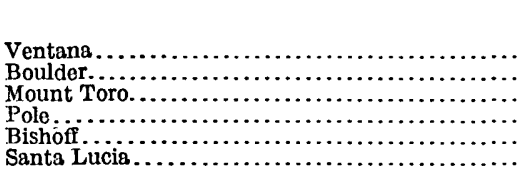 & $\begin{array}{rcc}\circ & 1 & \prime \prime \\
73 & 24 & 08.77 \\
112 & 53 & 45.91 \\
171 & 11 & 03.72 \\
213 & 11 & 10.08 \\
292 & 00 & 00.14 \\
323 & 45 & 29.24\end{array}$ & \begin{tabular}{rcc}
\multicolumn{1}{c}{$: " \prime$} & $\prime \prime$ \\
253 & 20 & 14.38 \\
292 & 45 & 56.55 \\
351 & 09 & 35.40 \\
33 & 13 & 52.96 \\
112 & 13 & 02.44 \\
143 & 50 & 46.95
\end{tabular} & \begin{tabular}{|r|} 
Log. meters. \\
4. 0132896 \\
4. 3309168 \\
4. 3829144 \\
4. 0971643 \\
4. 5520767 \\
4. 3566443
\end{tabular} & $\begin{array}{r}\text { Miles. } \\
6.407 \\
13.313 \\
15.006 \\
7.772 \\
22.153 \\
14.125\end{array}$ \\
\hline
\end{tabular}


CHOWCHILIA, MADERA COUNTY, CAIIF.

Station is the center of large steel water tank at the town of Chowchilla on the Southern Pacific R. R. The tank supplies the town with water.

[Latitude $37^{\circ} 07^{\prime} 34.27^{\prime \prime}$. Longitude $120^{\circ} 15^{\prime} 39.104^{\prime \prime}$.]

\begin{tabular}{|c|c|c|c|c|}
\hline To station- & Azimuth. & Back azimuth. & Dista & \\
\hline 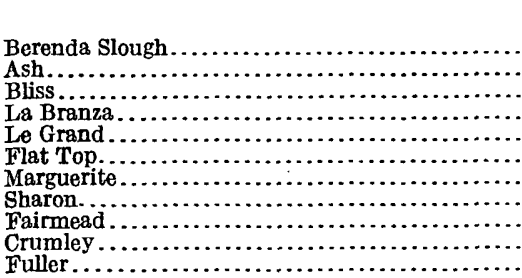 & $\begin{array}{rcc} & \prime \prime \prime \\
26 & 00 & 49.995 \\
63 & 00 & 03.56 \\
87 & 13 & 52.22 \\
135 & 54 & 03.62 \\
186 & 05 & 47.42 \\
219 & 32 & 30.19 \\
243 & 05 & 19.97 \\
283 & 53 & 53.22 \\
311 & 27 & 12.715 \\
325 & 16 & 17.00 \\
352 & 50 & 48.32\end{array}$ & $\begin{array}{rcc}\circ & \prime & \prime \prime \\
205 & 58 & 27.83 \\
242 & 55 & 47.35 \\
267 & 08 & 37.56 \\
315 & 51 & 51.96 \\
6 & 06 & 28.96 \\
39 & 38 & 57.84 \\
63 & 08 & 28.61 \\
103 & 58 & 35.69 \\
131 & 29 & 42.34 \\
145 & 19 & 40.99 \\
172 & 51 & 30.72\end{array}$ & $\begin{array}{r}\text { Log. meters. } \\
\text { 4. } 1235994 \\
4.0709244 \\
4.1101135 \\
3.8880722 \\
4.2025688 \\
4.3215165 \\
3.9367548 \\
4.0758752 \\
3.9124851 \\
4.1668480 \\
4.1452213\end{array}$ & $\begin{aligned} \text { Milcs. } \\
8.259 \\
7.316 \\
8.007 \\
4.802 \\
9.906 \\
13.028 \\
5.372 \\
7.400 \\
5.080 \\
9.124 \\
8.681\end{aligned}$ \\
\hline
\end{tabular}

CHRISTIAN, MERCED COUNTY, CALIF.

On highest summit of bald, round hill, locally known as Christian Hill, on land owned by the Simon Newman Co., about 7 miles southwest of town of Gustine, a station on the Southern Pacific R. R., and 0.5 mile west of ranch owned by J. F. Schnyder. Can be reached from Schnyder's ranch on horseback.

Station mark: A 4 by 4 inch redwood pole, 14 feet high, having three cross targets, and sunk 3 feet in the ground.

Reference mark: A bronze triangulation tablet, set in rock 4.6 feet northwest of signal and station mark. True azimuth, station to reference mark, $163^{\circ} 56^{\prime}$.

[Latitude $37^{\circ} 11^{\prime} 36.236^{\prime \prime}$ (1912), 37 $11^{\prime} 36.225^{\prime \prime}$ (1915). Longitude $121^{\circ} 05^{\prime} 38.142^{\prime \prime}$ (1912), $121^{\circ} 05^{\prime} 38.137^{\prime \prime}$ (19i5]).

\begin{tabular}{|c|c|c|c|c|}
\hline To station- & Azimuth. & Back azimuth. & \multicolumn{2}{|c|}{ Bistanco. } \\
\hline & $\circ \quad, \quad 1$ & - , & Log. meters. & Miles. \\
\hline Howard (1912). & $\begin{array}{llll}74 & 01 & 28.25\end{array}$ & 2535655.78 & 4.0633837 & 7.190 \\
\hline Orestimba (1912). & 1411609.99 & 3211124.08 & 4. 2691191 & 11.547 \\
\hline Cleveland (1912). & $158 \quad 49 \quad 17.23$ & $\begin{array}{lll}338 & 47 & 56.90\end{array}$ & 3. 9568293 & 5. 626 \\
\hline Crow $(1912) \ldots \ldots$ & 1652911.90 & $\begin{array}{lll}345 & 27 & 16.10\end{array}$ & 4. 2736010 & 11.667 \\
\hline Newman (1912). & 2044118.77 & $244 \quad 43 \quad 50.78$ & 4. 1704023 & 9.199 \\
\hline Gustine (1912). & $23036 \quad 21.94$ & 503943.78 & 4. 0269475 & 6.611 \\
\hline Stack (1912).... & 2603400.83 & $8038 \quad 10.83$ & 4. 0143355 & 6. 422 \\
\hline Barreillas (1912). & 3451130.55 & $165 \quad 13 \quad 30.43$ & 4. 2836434 & 11.940 \\
\hline$(1915) \ldots$ & 1652911.90 & $\begin{array}{llll}345 \quad 27 & 16.10\end{array}$ & 4. 2736020 & 11.667 \\
\hline South End Barn (1915 & $22906 \quad 36.52$ & $49 \quad 14 \quad 42.83$ & 4. 4174049 & 16. 246 \\
\hline Gustine $(1915) \ldots$ & $23036 \quad 25.84$ & 503947.69 & 4. 0269572 & 6.612 \\
\hline Fairmont (1915). & $257 \quad 1547.61$ & $\begin{array}{llll}77 & 23 & 45.43\end{array}$ & 4. 3003126 & 12.407 \\
\hline Underwood (1915). & $27014 \quad 01.90$ & 90.2317 .20 & 4. 3552129 & 14. 079 \\
\hline Ingomar (1915) ..... & $27734 \quad 42.96$ & 973917.07 & 4. 0525214 & 7.012 \\
\hline Milepost 162 (1 & $288 \quad 14 \quad 44.19$ & 1082312.31 & & 13.587 \\
\hline Los Banos Tank (1915) . . & $\begin{array}{lll}303 & 33 & 32.17\end{array}$ & 1234232.58 & 4. 4239815 & 16. 494 \\
\hline Romero (1915). & 3145631.66 & 1345922.97 & 3. 9951898 & 6.145 \\
\hline
\end{tabular}

CLOVIS, FRESNO COUNTY, CALIF. (Not occupied.)

Station is a large steel water tank in the town of Clovis, which is on the Southern Pacific R. R., about 9 miles northeast of Fresno.

[Latitude $36^{\circ} 49^{\prime} 26.265^{\prime \prime}$. Longitude $119^{\circ} 41^{\prime} 55.561^{\prime \prime}$.]

\begin{tabular}{|c|c|c|c|c|}
\hline To station- & Azimuth. & Back azimuth. & Distar & \\
\hline 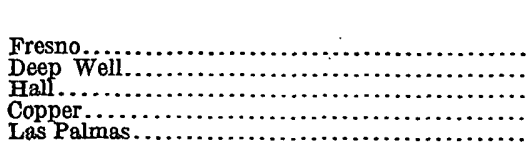 & $\begin{array}{ccc}\circ & \prime & \prime \prime \\
39 & 34 & 27.92 \\
127 & 24 & 11.11 \\
162 & 23 & 25.57 \\
201 & 27 & 44.31 \\
359 & 30 & 32.87\end{array}$ & \begin{tabular}{rcc}
\multicolumn{1}{c}{} & $\prime$ & $\prime \prime$ \\
219 & 31 & 15.51 \\
307 & 18 & 39.37 \\
342 & 21 & 30.56 \\
21 & 29 & 39.34 \\
179 & 30 & 38.21
\end{tabular} & \begin{tabular}{|r|} 
Log. meters. \\
4.0974228 \\
4.2362237 \\
4.1950005 \\
4.1128144 \\
3.8698088
\end{tabular} & $\begin{array}{r}\text { Miles. } \\
7.776 \\
10.705 \\
9.735 \\
8.057 \\
4.604\end{array}$ \\
\hline
\end{tabular}


COLUMBIA, MADERA COUNTY, CAIIF. (Not occupied.)

Station mark: Center of windmill at Miller \& Lux Columbia ranch house, east of Firebaugh.

[Latitude $36^{\circ} 50^{\prime} 50.197^{\prime \prime}$. Longitude $120^{\circ} 21^{\prime} 29.842^{\prime \prime}$.]

\begin{tabular}{|c|c|c|c|c|}
\hline Tostation- & Azimuth. & Back azimuth. & \multicolumn{2}{|c|}{ Distance. } \\
\hline 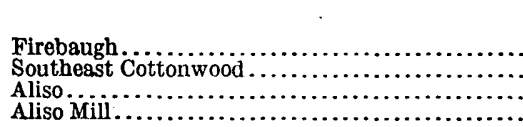 & $\begin{array}{ccc}\circ & \prime & \prime \prime \\
99 & 08 & 49.02 \\
222 & 48 & 46.00 \\
232 & 24 & 27.10 \\
260 & 02 & 06.82\end{array}$ & \begin{tabular}{rcc}
\multicolumn{1}{c}{} & $\prime$ & $\prime \prime$ \\
279 & 05 & 14.98 \\
42 & 52 & 38.91 \\
52 & 26 & 00.04 \\
80 & 05 & 51.98
\end{tabular} & $\begin{array}{r}\text { Log. meters. } \\
\text { 3. } 9520097 \\
4.1424330 \\
3.6851039 \\
3.9671745\end{array}$ & $\begin{aligned} \text { Miles. } \\
\text { 5. } 564 \\
\text { 8. } 625 \\
\text { 3. } 009 \\
\text { 5. } 761\end{aligned}$ \\
\hline
\end{tabular}

COPPER, FRESNO COUNTY, CALIF.

On a high foothill, 8 miles in an air line north-northeast of Clovis. Follow road from Clovis to Copper Mine power station on the San Joaquin Light \& Power Co.'s line, thence go east through gate into pasture to first ranch house, thence to spring watering trough near foot of mountain. Buckboards can not be driven to summit.

Signal: Cairn on most northern point of hill.

Station mark: Tablet under cairn.

[Latitude $36^{\circ} 55^{\prime} 57.685^{\prime \prime}$ (1915), $36^{\circ} 55^{\prime} 58.57^{\prime \prime}$ (1908). Longitude $119^{\circ} 38^{\prime} 43.860^{\prime \prime}$ (1915), $119^{\circ} 38^{\prime} 42.97^{\prime \prime}$ (1908).]

\begin{tabular}{|c|c|c|c|c|}
\hline To station- & Azimuth. & Back azimuth. & Distar & ace. \\
\hline 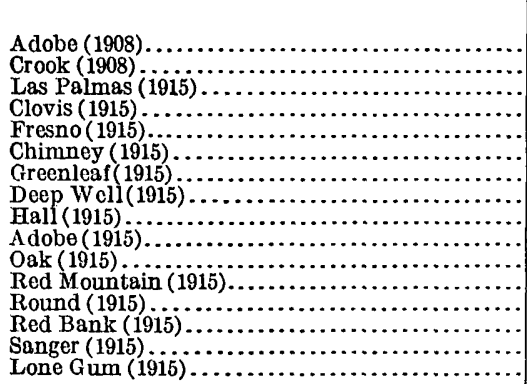 & \begin{tabular}{rcc}
\multicolumn{1}{c}{} & $\prime$ & $\prime \prime$ \\
120 & 25 & 30.71 \\
189 & 15 & 02.57 \\
13 & 32 & 42.05 \\
21 & 29 & 39.34 \\
30 & 23 & 18.70 \\
46 & 36 & 03.82 \\
77 & 37 & 17.12 \\
85 & 03 & 41.45 \\
106 & 50 & 50.73 \\
120 & 26 & 02.75 \\
255 & 09 & 18.82 \\
289 & 15 & 33.91 \\
316 & 03 & 47.42 \\
341 & 31 & 42.04 \\
342 & 11 & 51.66 \\
355 & 10 & 26.76
\end{tabular} & \begin{tabular}{rcc}
\multicolumn{1}{c}{0} & $\prime$ & $\prime \prime$ \\
300 & 17 & 43.21 \\
9 & 16 & 04.24 \\
193 & 30 & 48.63 \\
201 & 27 & 44.31 \\
210 & 18 & 11.12 \\
226 & 30 & 01.10 \\
257 & 26 & 49.03 \\
264 & 56 & 14.12 \\
286 & 47 & 00.34 \\
300 & 18 & 15.16 \\
75 & 13 & 15.78 \\
109 & 24 & 26.58 \\
136 & 08 & 41.18 \\
161 & 33 & 20.54 \\
162 & 15 & 04.29 \\
175 & 10 & 43.91
\end{tabular} & $\begin{array}{r}\text { Log. meters. } \\
4.3477863 \\
4.1971765 \\
4.3017111 \\
4.1128144 \\
4.4007709 \\
4.3144082 \\
4.4235469 \\
4.2671488 \\
3.9959738 \\
4.3479198 \\
4.0039318 \\
4.3669627 \\
4.2426860 \\
4.1085492 \\
4.4165379 \\
3.9249400\end{array}$ & $\begin{array}{r}\text { Miles. } \\
\ldots . . . . . . \\
\ldots . . . . \\
12.447 \\
8.057 \\
15.636 \\
12.816 \\
16.478 \\
11.495 \\
6.156 \\
13.844 \\
6.270 \\
14.465 \\
10.865 \\
7.978 \\
16.214 \\
5.227\end{array}$ \\
\hline
\end{tabular}

CROW, STANISLaUs CoUNTY, CaLIF.

On highest point of round foothill 5 miles southwest of Crows Landing, just south of Crow Creek, on land owned by George Crow, and 1.5 miles north of his ranch house. Can drive to foot of hill from almost any direction.

Station mark and signal: A 4 by 4 inch redwood pole, 10 feet long, having 3-foot cross targets, and set 3 feet in the ground.

Reference mark: A bronze triangulation tablet set in rock 3.9 feet south of signal. True azimuth from station to reference mark, $355^{\circ} 11^{\prime}$.

[Latitude $37^{\circ} 21^{\prime} 25.814^{\prime \prime}$. Longitude $121^{\circ} 08^{\prime} 49.339^{\prime \prime}$.]

\begin{tabular}{|c|c|c|c|c|}
\hline To station- & Azimuth. & Back azimuth. & Distal & \\
\hline 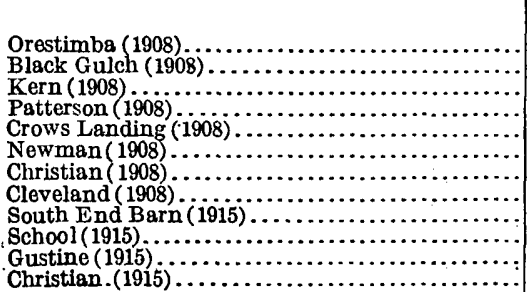 & $\begin{array}{rcc}\circ & \prime & \prime \prime \\
61 & 58 & 05.31 \\
146 & 31 & 47.61 \\
154 & 46 & 22.64 \\
185 & 50 & 14.80 \\
239 & 24 & 05.94 \\
293 & 25 & 33.12 \\
345 & 27 & 16.10 \\
351 & 35 & 06.66 \\
272 & 27 & 04.59 \\
279 & 33 & 20.85 \\
311 & 25 & 58.53 \\
345 & 27 & 16.10\end{array}$ & \begin{tabular}{rcc}
\multicolumn{1}{c}{} & $\prime$ & $\prime \prime$ \\
241 & 55 & 14.85 \\
326 & 28 & 58.19 \\
334 & 43 & 24.32 \\
5 & 50 & 46.97 \\
59 & 26 & 47.20 \\
113 & 30 & 01.38 \\
165 & 29 & 11.90 \\
171 & 35 & 42.08 \\
92 & 37 & 07.81 \\
99 & 42 & 01.03 \\
131 & 31 & 16.64 \\
165 & 29 & 11.90
\end{tabular} & \begin{tabular}{c|} 
Log. meters. \\
3.8942543 \\
4.0944841 \\
4.2281635 \\
4.1070391 \\
3.8803463 \\
4.0744560 \\
4.2736010 \\
3.9929778 \\
4.3890577 \\
4.3306488 \\
4.2368543 \\
4.2736020
\end{tabular} & $\begin{array}{r}\text { Miles. } \\
4.871 \\
7.724 \\
10.508 \\
7.950 \\
4.717 \\
7.376 \\
11.667 \\
6.114 \\
15.220 \\
13.305 \\
10.720 \\
11.687\end{array}$ \\
\hline
\end{tabular}




\section{CRUMLEY, MADERA COUNTY, CALIF.}

On the southwest bank of a reservoir 400 feet southwest of Mr. Crumley's house, which is about 2 miles southwest of Berenda, a station on the Southern Pacific R. R. between Merced. and Madera.

Signal: Lumber structure with cross targets.

Station mark: Iron bench mark centered under signal.

[Latitude $37^{\circ} 01^{\prime} 02.666^{\prime \prime}$. Longitude $120^{\circ} 10^{\prime} 00.704^{\prime \prime}$.]

\begin{tabular}{|c|c|c|c|c|}
\hline To station- & Azimuth. & Back azimuth. & Distar & \\
\hline 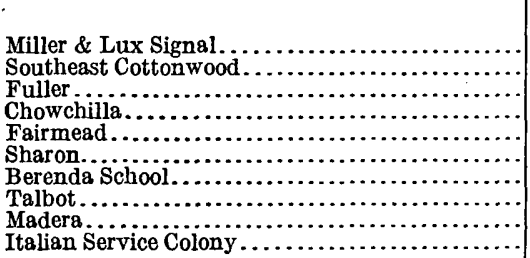 & $\begin{array}{rcc}1 & 37 & 21.78 \\
41 & 11 & 02.99 \\
74 & 54 & 29.19 \\
145 & 19 & 40.99 \\
161 & 28 & 13.69 \\
199 & 11 & 33.52 \\
208 & 56 & 01.75 \\
261 & 07 & 30.62 \\
303 & 57 & 40.42 \\
330 & 26 & 08.42\end{array}$ & \begin{tabular}{rcc}
\multicolumn{1}{c}{} & $\prime$ & $\prime \prime$ \\
181 & 37 & 15.43 \\
221 & 07 & 57.90 \\
254 & 51 & 47.84 \\
325 & 16 & 17.00 \\
341 & 27 & 19.25 \\
19 & 12 & 51.71 \\
28 & 56 & 32.28 \\
81 & 12 & 31.13 \\
124 & 01 & 29.82 \\
150 & 28 & 18.92
\end{tabular} & \begin{tabular}{r|} 
Log. meters. \\
3.9652420 \\
4.0630925 \\
3.8365911 \\
4.1668480 \\
3.8465001 \\
3.9888415 \\
3.4133365 \\
4.0963184 \\
4.0558576 \\
4.0367511
\end{tabular} & $\begin{array}{r}\text { Miles. } \\
\text { 5.736' } \\
7.185 \\
4.265 \\
9.124 \\
4.364 \\
6.056 \\
1.609 \\
7.757 \\
7.067 \\
\mathbf{6 . 7 6 2}\end{array}$ \\
\hline
\end{tabular}

\section{DAULTON, MADERA COUNTY, CALTF.}

On a hill on which are scattered scrubby oaks, about 1 mile north of Daulton station.

Signal: Flag in southern one of two trees on highest point. There is a cross on rock just south of tree.

Station mark: Tablet 37 feet west from signal.

Position of signal computed. Azimuth from tablet to signal, $242^{\circ} 54^{\prime}$; distance, 37.2 feet.

[Latitude $37^{\circ} 07^{\prime} 55.785^{\prime \prime}$. Longitude $119^{\circ} 58^{\prime} 58.006^{\prime \prime}$.]

\begin{tabular}{|c|c|c|c|c|}
\hline To station- & Azimuth. & Back azimuth. & Dist & nce. \\
\hline 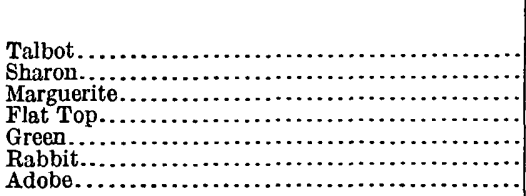 & \begin{tabular}{rcc}
\multicolumn{1}{c}{} & $\prime$ & $\prime \prime$ \\
20 & 30 & 08.46 \\
75 & 01 & 18.90 \\
100 & 52 & 19.07 \\
143 & 52 & 27.87 \\
177 & 24 & 01.20 \\
263 & 37 & 59.82 \\
315 & 09 & 25.36
\end{tabular} & \begin{tabular}{rcc}
\multicolumn{1}{c}{} & $\prime$ & $\prime \prime$ \\
200 & 28 & 29.77 \\
254 & 55 & 57.30 \\
280 & 45 & 23.21 \\
323 & 47 & 50.27 \\
357 & 23 & 44.70 \\
83 & 39 & 53.11 \\
135 & 13 & 48.96
\end{tabular} & \begin{tabular}{|r|} 
Log. meters. \\
4.0625406 \\
4.1342778 \\
4.2381142 \\
4.2830273 \\
4.1713441 \\
3.6684214 \\
4.1853125
\end{tabular} & $\begin{array}{r}\text { Miles. } \\
7.176 \\
8.465 \\
10.751 \\
11.923 \\
9.219 \\
2.896 \\
9.521\end{array}$ \\
\hline
\end{tabular}

DEEP WELL, MADERA COUNTY, CALIF.

On north and south fence, 0.5 mile south of Madera and Friant road, at a point 1 mile more or less west of the Deep Well School.

Signal: Lumber structure with cross targets.

Station mark: Iron bench mark centered under signal.

[Latitude $36^{\circ} 55^{\prime} 05.375^{\prime \prime}$. Longitude $119^{\circ} 51^{\prime} 08.444^{\prime \prime}$.]

\begin{tabular}{|c|c|c|c|c|}
\hline To station- & Azimuth. & Back azimuth. & Distar & \\
\hline 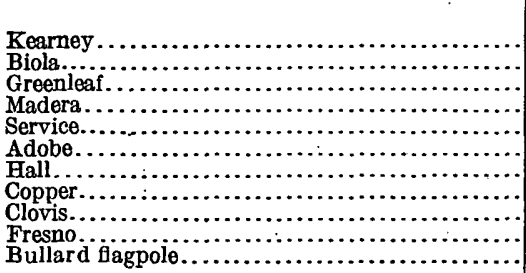 & $\begin{array}{rcc}\bullet & \prime & \prime \prime \\
15 & 09 & 21.55 \\
48 & 03 & 30.59 \\
61 & 09 & 57.00 \\
104 & 07 & 08.92 \\
131 & 40 & 30.58 \\
176 & 24 & 35.75 \\
243 & 21 & 41.64 \\
264 & 56 & 14.12 \\
307 & 18 & 39.37 \\
344 & 03 & 03.43 \\
347 & 13 & 53.11\end{array}$ & \begin{tabular}{rcc}
\multicolumn{1}{c}{} &, & $\prime \prime$ \\
195 & 07 & 07.43 \\
227 & 57 & 47.61 \\
241 & 06 & 56.07 \\
283 & 59 & 37.46 \\
311 & 38 & 01.94 \\
356 & 24 & 16.08 \\
63 & 25 & 18.76 \\
85 & 03 & 41.45 \\
127 & 24 & 11.11 \\
164 & 05 & 22.21 \\
167 & 14 & 35.42
\end{tabular} & $\begin{array}{r}\text { Log. meters. } \\
\text { 4. } 3523742 \\
4.2900607 \\
3.9303794 \\
4.2824698 \\
3.9133288 \\
4.1106596 \\
3.9999819 \\
4.2671488 \\
4.2362237 \\
4.3202756 \\
3.8977887\end{array}$ & $\begin{array}{r}\text { Miles. } \\
13.987 \\
12.117 \\
5.293 \\
11.907 \\
5.090 \\
8.017 \\
6.213 \\
11.495 \\
10.705 \\
12.990 \\
4.911\end{array}$ \\
\hline
\end{tabular}


DELTA, MERCED COUNTY, CALIF.

Station is the center of water tank at the Miller \& Lux Delta ranch, about 7 miles northeast of Los Banos, a town on the Southern Pacific R. R.

[Latitude $37^{\circ} 07^{\prime} 02.919^{\prime}$. Longitude $120^{\circ} 44^{\prime} 21.145^{\prime \prime}$.]

\begin{tabular}{|c|c|c|c|c|}
\hline To station- & Azimuth. & Back azimuth. & Dista & \\
\hline 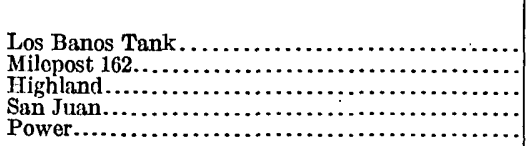 & \begin{tabular}{rcc|}
\multicolumn{1}{c}{} & $\prime$ & $\prime \prime$ \\
56 & 22 & 06.81 \\
98 & 15 & 23.27 \\
154 & 57 & 41.65 \\
298 & 59 & 57.09 \\
345 & 13 & 46.24
\end{tabular} & $\begin{array}{lcc}\circ & \prime & \prime \prime \\
236 & 18 & 16.64 \\
278 & 11 & 00.31 \\
334 & 56 & 43.03 \\
119 & 02 & 56.36 \\
165 & 14 & 30.62\end{array}$ & $\begin{array}{r}\text { Log. meters. } \\
\text { 4. } 0540401 \\
4.0360915 \\
\text { 3. } 7527904 \\
3.9239558 \\
3.8533472\end{array}$ & $\begin{array}{r}\text { Miles. } \\
7.037 \\
6.752 \\
3.517 \\
5.216 \\
4.433\end{array}$ \\
\hline
\end{tabular}

DERRICK, FRESNO COUNTY, CALIF.

Oil-well derrick 200 feet southwest of a levee connecting Fish and Fresno sloughs and about 3 miles east of San Joaquin, a town on the Hanford \& Summit Lake branch of the Southern Pacific R. R.

Signal: 100-foot oil derrick.

Reference mark: Center of 8-inch casing of deep well, 1 foot south of center of signal.

[Latitude $36^{\circ} 36^{\prime} 41.406^{\prime \prime}$. Longitude $120^{\circ} 08^{\prime} 17.405^{\prime \prime}$.]

\begin{tabular}{|c|c|c|c|c|}
\hline To station- & Azimuth. & Back azimuth. & Dista & co. \\
\hline 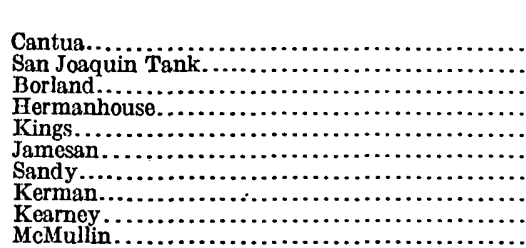 & $\begin{array}{rcc}- & \prime & \prime \prime \\
62 & 39 & 20.40 \\
83 & 07 & 06.39 \\
113 & 43 & 21.33 \\
133 & 13 & 52.11 \\
135 & 19 & 55.19 \\
156 & 25 & 30.78 \\
198 & 36 & 05.64 \\
209 & 12 & 20.41 \\
237 & 54 & 21.32 \\
250 & 56 & 35.93\end{array}$ & \begin{tabular}{rcc}
\multicolumn{1}{c}{} & $\prime$ & $\prime \prime$ \\
242 & 30 & 52.34 \\
263 & 05 & 18.87 \\
293 & 36 & 32.91 \\
313 & 07 & 30.17 \\
315 & 16 & 44.34 \\
336 & 23 & 07.66 \\
18 & 36 & 34.98 \\
29 & 15 & 07.64 \\
58 & 02 & 14.14 \\
71 & 02 & 55.60
\end{tabular} & $\begin{array}{r}\text { Log. meters. } \\
\text { 4. } 3782577 \\
3.6544898 \\
4.2685567 \\
4.3378948 \\
\text { 4. } 0528116 \\
\text { 4. } 1722098 \\
3.5830924 \\
4.1535698 \\
4.3653550 \\
4.1996952\end{array}$ & $\begin{array}{r}\text { Miles. } \\
14.846 \\
2.804 \\
11.532 \\
13.528 \\
7.017 \\
9.238 \\
2.379 \\
8.850 \\
14.411 \\
9.841\end{array}$ \\
\hline
\end{tabular}

DINUBA, FRESNO COUNTY, CALIF. (Not occupied.)

Station mark: Center of high red wooden tank in Dinuba.

[Latitude $36^{\circ} 32^{\prime} 35.956^{\prime \prime}$. Longitude $119^{\circ} 23^{\prime} 09.989^{\prime \prime}$.]

\begin{tabular}{|c|c|c|c|c|}
\hline To station- & Azimuth. & Back azimuth. & Distar & \\
\hline 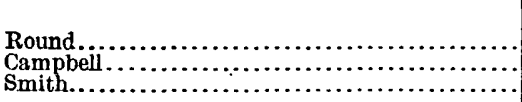 & $\begin{array}{ccc}\circ & \prime & \prime \prime \\
160 & 12 & 53.79 \\
169 & 32 & 55.96 \\
220 & 12 & 10.64\end{array}$ & \begin{tabular}{rcc}
\multicolumn{1}{c}{} & \multicolumn{1}{c}{$\prime \prime$} \\
340 & 08 & 32.00 \\
349 & 31 & 40.94 \\
40 & 13 & 38.20
\end{tabular} & $\begin{array}{r}\text { Log. meters. } \\
\text { 4. } 5123564 \\
\text { 4. } 2357838 \\
\text { 3. } 7529353\end{array}$ & $\begin{array}{r}\text { Miles. } \\
20.217 \\
10.694 \\
3.518\end{array}$ \\
\hline
\end{tabular}

DOS PALOS, FRESNO COUNTY, CALIF.

Center of tank house supplying water to Dos Palos.

Signal: Flagpole on top of tank house.

Station mark: Artesian well in center of tank house.

[Latitude $36^{\circ} 59^{\prime} 14.169^{\prime \prime}$. Longitude $120^{\circ} 37^{\prime} 31.018^{\prime \prime}$.]

\begin{tabular}{|c|c|c|c|c|}
\hline Tostation- & Azimuth. & Back azimuth. & Distan & \\
\hline 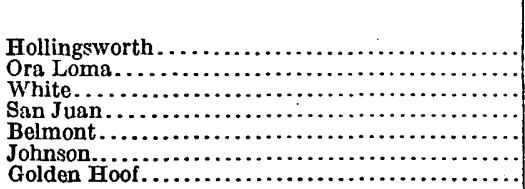 & $\begin{array}{rcc}* & , & \prime \prime \\
4 & 02 & 48.36 \\
32 & 07 & 27.71 \\
66 & 58 & 01.41 \\
164 & 57 & 33.16 \\
181 & 53 & 56.30 \\
229 & 10 & 14.95 \\
288 & 04 & 39.19\end{array}$ & 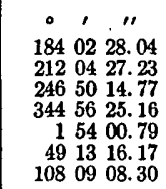 & $\begin{array}{r}\text { Log. meters. } \\
4.0744041 \\
4.1457490 \\
4.3197460 \\
4.0312537 \\
3.7452289 \\
4.1165906 \\
4.0661607\end{array}$ & $\begin{array}{r}\text { Miles. } \\
7.375 \\
8.692 \\
12.975 \\
6.677 \\
3.456 \\
8.127 \\
7.236\end{array}$ \\
\hline
\end{tabular}




\section{DRESSER, SAN LUIS OBISPO COUNTY, CALIF.}

On a small hill about 8 miles east-southeast of Paso Robles and on land owned by Mr. Dresser. From Paso Robles take the Creston road until first schoolhouse is passed (about 7 miles), continue on same road to first ranch on left (1 mile), then turn north up lane to summit of ridge, thence go through a fence and east along ridge until highest point is reached. All oak trees were cut on the summit. The next hill east of this point is bald but lower than the point used.

Signal: An oak tree planted in the ground on the highest point.

Station mark: A triangulation tablet set in rock. Position of tablet computed. Azimuth from tablet to signal, $209^{\circ} 35^{\prime}$; distance, 0.45 meter.

[Latitude $35^{\circ} 34^{\prime} 55.762^{\prime \prime}$. Longitude $120^{\circ} 34^{\prime} 20.737^{\prime \prime}$.]

\begin{tabular}{|c|c|c|c|c|}
\hline To station- & Azimuth. & Back azimuth. & Dista & \\
\hline 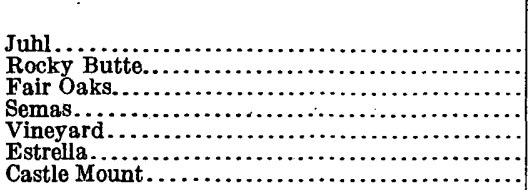 & \begin{tabular}{rcc}
\multicolumn{1}{c}{} & $\prime$ & $\prime \prime$ \\
70 & 34 & 26.80 \\
102 & 00 & 00.26 \\
108 & 58 & 09.39 \\
146 & 13 & 51.66 \\
157 & 58 & 18.82 \\
195 & 41 & 38.63 \\
207 & 54 & 36.29
\end{tabular} & \begin{tabular}{|rcc}
\multicolumn{1}{c}{$\circ$} & $\prime$ & $\prime \prime$ \\
250 & 25 & 12.97 \\
281 & 43 & 00.18 \\
288 & 50 & 25.90 \\
326 & 05 & 03.50 \\
337 & 54 & 46.84 \\
15 & 43 & 27.76 \\
28 & 02 & 45.92
\end{tabular} & $\begin{array}{r}\text { Log. meters. } \\
4.4058368 \\
4.6535228 \\
4.3258150 \\
4.6107150 \\
4.3861901 \\
4.2403727 \\
4.6519052\end{array}$ & $\begin{array}{r}\text { Miles. } \\
15.819 \\
27.982 \\
13.157 \\
25.355 \\
15.120 \\
10.807 \\
27.878\end{array}$ \\
\hline
\end{tabular}

DUNRARD, FRESNO COUNTY, CALIF. (Not occupied.)

Station mark: Center of cupola of Dunkard School.

[Latitude $36^{\circ} 42^{\prime} 49.875^{\prime \prime}$. Longitude $119^{\circ} 59^{\prime} 46.616^{\prime \prime}$.]

\begin{tabular}{|c|c|c|c|c|}
\hline To station- & Azimuth. & Back azimuth. & Distan & \\
\hline 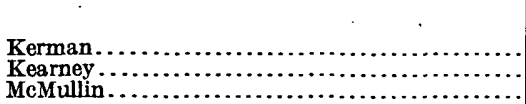 & $\begin{array}{ccc}\circ & \prime & \prime \prime \\
100 & 36 & 36.21 \\
262 & 16 & 53.12 \\
339 & 46 & 33.74\end{array}$ & \begin{tabular}{rcc}
\multicolumn{1}{c}{} & $\prime$ & $\prime \prime$ \\
280 & 34 & 18.25 \\
82 & 19 & 41.12 \\
159 & 47 & 28.68
\end{tabular} & \begin{tabular}{|} 
Log. meters. \\
3.7653452 \\
3.8473695 \\
3.8201001
\end{tabular} & $\begin{array}{r}\text { Miles. } \\
3.620 \\
4.372 \\
4.106\end{array}$ \\
\hline
\end{tabular}

EADE, MONTEREY COUNTY, CALIF.

On flat ridge 8 or 9 miles east of King City. It can be reached by taking Lonoak road from King City for about 5 miles, thence turn to right and go up canyon passing Herman Eade's ranch to forks of road; thence take right-hand road and continue up canyon until oak trees on right -hand side of slope of ridge are reached. Signal is on top of ridge to south. Can continue up canyon to top of ridge and drive back on ridge to station, but 2 miles will be saved by walking up.

Signal: An oak tree trimmed.

Station mark: A triangulation tablet in rock 1.7 meters from the signal tree; azimuth from signal to tablet, $149^{\circ}$.

[Latitude $36^{\circ} 13^{\prime} 31.688^{\prime \prime}(1917), 36^{\circ} 13^{\prime} 31.91^{\prime \prime}$ (1909). Longitude $120^{\circ} 58^{\prime} 08.997^{\prime \prime}$ (1917), $120^{\circ} 58^{\prime} 09.20$ (1909).]

\begin{tabular}{|c|c|c|c|c|}
\hline To station- & Azimuth. & Back azimuth. & Dista & nce. \\
\hline 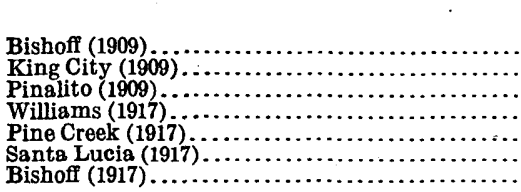 & \begin{tabular}{rcc}
$\circ$ & $\prime$ & \multicolumn{1}{c}{} \\
79 & 26 & 01.25 \\
86 & 37 & 14.23 \\
135 & 48 & 10.90 \\
5 & 23 & 40.36 \\
59 & 51 & 58.55 \\
77 & 48 & 34.75 \\
79 & 26 & 08.61
\end{tabular} & $\begin{array}{ccc}\circ & \prime & \prime \prime \\
259 & 17 & 50.12 \\
266 & 31 & 40.74 \\
315 & 44 & 19.63 \\
185 & 22 & 32.97 \\
239 & 42 & 49.12 \\
257 & 32 & 40.11 \\
259 & 17 & 57.41\end{array}$ & \begin{tabular}{|l} 
Log. meters. \\
4. 3249351 \\
4.1498586 \\
4.1457788 \\
4.4842617 \\
4.4302126 \\
4.6165346 \\
4.3250001
\end{tabular} & 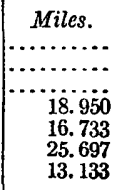 \\
\hline
\end{tabular}


EASTIN SCHOOL, MADERA COUNTY, CALIF.

Signal and station mark: Cupola of Eastin School, about 6 miles south and 1 mile east of Madera.

[Latitude $36^{\circ} 51^{\prime} 58.952^{\prime \prime}$. Longitude $\left.120^{\circ} 00^{\prime} 33.214^{\prime \prime}.\right]$

\begin{tabular}{|c|c|c|c|c|}
\hline To station- & Azimuth. & Back azimuth. & Dista1 & \\
\hline 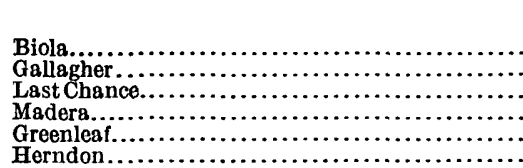 & $\begin{array}{rcc}\circ & , & \prime \prime \\
3 & 57 & 34.34 \\
55 & 46 & 21.12 \\
87 & 51 & 11.42 \\
156 & 05 & 12.00 \\
255 & 54 & 34.49 \\
290 & 13 & 26.25\end{array}$ & \begin{tabular}{rcc}
\multicolumn{1}{c}{} & $\prime$ & $\prime \prime$ \\
183 & 57 & 22.10 \\
235 & 42 & 32.50 \\
267 & 45 & 57.19 \\
336 & 03 & 20.02 \\
75 & 57 & 12.56 \\
110 & 16 & 50.16
\end{tabular} & \begin{tabular}{|r|} 
Log. meters. \\
3.8643524 \\
4.0581272 \\
4.1134044 \\
4.0561051 \\
3.8277556 \\
3.9531716
\end{tabular} & $\begin{array}{r}\text { Miles. } \\
4.547 \\
7.104 \\
8.068 \\
7.071 \\
4.179 \\
5.579\end{array}$ \\
\hline
\end{tabular}

EAST STACK, FRESNO COUNTY, CALIF. (Not occupled.)

Station mark: Eastern one of two stacks at pump station, west of Standard Oil (Mendota) tanks pump station. ${ }^{1}$

[Latitude $36^{\circ} 36^{\prime} 08.599^{\prime \prime}$. Longitude $120^{\circ} 24^{\prime} 19.293^{\prime \prime}$.]

\begin{tabular}{|c|c|c|c|c|}
\hline To station- & Azimuth. & Back azimuth. & Dis & nce. \\
\hline 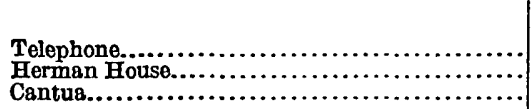 & $\begin{array}{ccc}\circ & , & \prime \prime \\
135 & 33 & 47.10 \\
206 & 41 & 15.81 \\
344 & 48 & 34.08\end{array}$ & $\begin{array}{rcc}\circ & \prime & \prime \prime \\
315 & 29 & 52.69 \\
26 & 44 & 28.41 \\
164 & 49 & 39.00\end{array}$ & $\begin{array}{r}\text { Log. meters. } \\
4.1438113 \\
4.2506831 \\
4.0149715\end{array}$ & $\begin{array}{r}\text { Miles. } \\
8.653 \\
11.067 \\
6.432\end{array}$ \\
\hline
\end{tabular}

ELKHORN, SAN BENITO COUNTY, CALIF.

On the south end of a high hill, which has oak trees on north end and is bald on south end, about 3 miles northeast of Elkhorn. Station is owned by T. S. Ingels, who lives 14 miles from Tres Pinos, on the New Idria road. It can be reached from Elkhorn by going on steep road to Mr. Joseph Thomas's ranch, thence on horseback to summit of hill. Land on which station is situated is owned by Mr. Thomas.

Signal: An oak tree set 3 feet in the ground.

Station mark: A U. S. G. S. triangulation tablet under center of station. Position of tablet computed.

[Latitude $36^{\circ} 41^{\prime} 34.045 .^{\prime \prime}$ Longitude $\left.121^{\circ} 08^{\prime} 34.043 .^{\prime \prime}\right]$

\begin{tabular}{|c|c|c|c|c|}
\hline To station- & Azimuth. & Back azimuth. & Distar & ce. \\
\hline 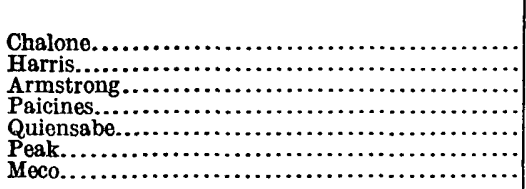 & \begin{tabular}{rcc}
\multicolumn{1}{c}{} & $\prime$ & $\prime \prime$ \\
9 & 46 & 15.24 \\
24 & 10 & 25.50 \\
59 & 28 & 21.12 \\
115 & 27 & 28.12 \\
155 & 44 & 29.70 \\
182 & 27 & 07.02 \\
228 & 55 & 45.05
\end{tabular} & \begin{tabular}{rcc}
\multicolumn{1}{c}{} & $\prime$ & $\prime \prime$ \\
189 & 44 & 23.36 \\
204 & 07 & 37.81 \\
239 & 22 & 13.21 \\
295 & 12 & 36.26 \\
335 & 41 & 46.65 \\
2 & 27 & 21.83 \\
48 & 58 & 49.34
\end{tabular} & \begin{tabular}{r|} 
Log. meters. \\
4. 4402035 \\
4. 2322725 \\
4. 2499493 \\
4.2085915 \\
4. 2157235 \\
4.1565683 \\
4.0060857
\end{tabular} & $\begin{array}{r}\text { Miles. } \\
\text { 17. } 122 \\
10.608 \\
11.048 \\
10.045 \\
10.211 \\
8.911 \\
6.301\end{array}$ \\
\hline
\end{tabular}

EL NIDO, MERCED COUNTY, CALIF. (Not occupled.)

Station is center of windmill tower at the El Nido School, about 10 miles west of Chowchilla, a town on the Southern Pacific R. R.

[Latitude $37^{\circ} 08^{\prime} 03.961^{\prime \prime}$. Longitude $120^{\circ} 29^{\prime} 27.187^{\prime \prime}$.]

\begin{tabular}{|c|c|c|c|c|}
\hline To station- & Azimuth. & Back azimuth. & Distan & \\
\hline 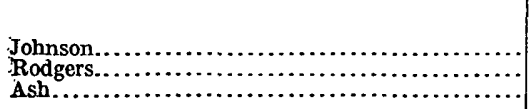 & \begin{tabular}{rcc}
\multicolumn{1}{c}{} & \multicolumn{1}{c}{ ' } & \multicolumn{1}{c}{14} \\
14 & 47 & 42.07 \\
75 & 56 & 05.06 \\
302 & 07 & 41.59
\end{tabular} & \begin{tabular}{ccc|}
$\circ$ & $\prime$ & $\prime \prime$ \\
194 & 46 & 51.86 \\
255 & 54 & 56.92 \\
122 & 11 & 44.83
\end{tabular} & $\begin{array}{c}\text { Log. meter } 8 . \\
\text { 3. } 9059507 \\
\text { 3. } 4582780 \\
\text { 4. } 0704060\end{array}$ & $\begin{array}{r}\text { Miles. } \\
5.004 \\
1.785 \\
7.307\end{array}$ \\
\hline
\end{tabular}

1 Thesestacks havesince been destroyed. 
ESTRELLA, SAN LUIS OBISPO COUNTY, CALIF.

On a bald hill on the Estrella ranch, about 15 miles from San Miguel. Go from San Miguel by Estrella post office to Bern post office, thence east and north over small ridge to Freeman Canyon, and up this canyon until a gate is reached. Follow the road through this gate until it crosses the canyon, then turn west. An orchard and water trough can be seen on the west side of the fence. Go through a gate at this place thence northwest up ridge to signal. A buckboard or Ford car can be driven to signal.

Signal: An oak tree set in the ground.

Station mark: A rock with chiseled cross on it. No tablet was set in this rock, as it was very hard and broke both drills. There is no other rock on the hill, and this one had to be hauled there. Position of mark was computed. Azimuth from mark to signal, $56^{\circ} 14^{\prime}$; distance, 0.60 meter.

[Latitude $35^{\circ} 43^{\prime} 59.033^{\prime \prime}$. Longitude $120^{\circ} 31^{\prime} 13.520^{\prime \prime}$.]

\begin{tabular}{|c|c|c|c|c|}
\hline To station- & Azimuth. & Back azimuth. & Distan & \\
\hline 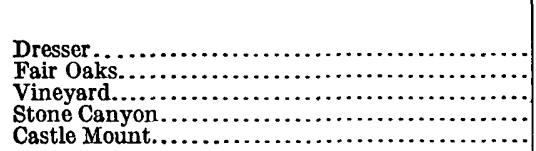 & \begin{tabular}{rcc}
\multicolumn{1}{c}{} & $\prime$ & $\prime \prime$ \\
15 & 43 & 27.76 \\
68 & 17 & 22.55 \\
112 & 49 & 22.33 \\
174 & 45 & 31.19 \\
215 & 27 & 50.43
\end{tabular} & $\begin{array}{ccc}\circ & \prime & \prime \prime \\
195 & 41 & 38.63 \\
248 & 07 & 48.99 \\
292 & 44 & 00.56 \\
354 & 47 & 22.27 \\
35 & 34 & 11.34\end{array}$ & \begin{tabular}{|r|} 
Log. meters. \\
4.2403727 \\
4.4252600 \\
4.1761640 \\
4.5123908 \\
4.4488243
\end{tabular} & $\begin{array}{r}\text { Miles. } \\
10.807 \\
16.543 \\
9.322 \\
20.218 \\
17.465\end{array}$ \\
\hline
\end{tabular}

EXRAND, MERCED COUNTY, CALIF.

High cement silo on the Eyrand ranch, about 7 miles southeast of Atwater, and about 10 miles southwest of Merced. To reach it go south from Merced 2 miles, thence west 4 miles, thence south 4 miles.

Signal and station mark: Center of cement silo about 50 feet high. It can be occupied.

[Latitude $37^{\circ} 13^{\prime} 39.373^{\prime \prime}$. Longitude $120^{\circ} 33^{\prime} 12.086^{\prime \prime}$.]

\begin{tabular}{|c|c|c|c|c|}
\hline Tostation- & Azimuth. & Back azimuth. & Dist & nce. \\
\hline 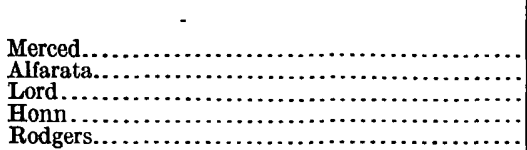 & $\begin{array}{ccc}\circ & \prime & \prime \prime \\
215 & 11 & 47.93 \\
241 & 45 & 12.13 \\
295 & 48 & 17.73 \\
317 & 32 & 57.40 \\
345 & 55 & 31.82\end{array}$ & \begin{tabular}{rcc}
\multicolumn{1}{c}{} & $\prime$ & $\prime \prime$ \\
35 & 14 & 20.54 \\
61 & 49 & 13.55 \\
115 & 52 & 11.55 \\
137 & 36 & 31.72 \\
165 & 56 & 52.64
\end{tabular} & $\begin{array}{r}\text { Log. meters. } \\
4.0321314 \\
4.0650404 \\
4.0250777 \\
4.1128238 \\
4.0564102\end{array}$ & $\begin{array}{r}\text { Miles. } \\
6.691 \\
7.218 \\
6.583 \\
8.057 \\
7.076\end{array}$ \\
\hline
\end{tabular}

FAIRMEAD, MADERA COUNTY, CALIF. (Not occupied.)

Station is center of steel water tank at town of Fairmead, on the Southern Pacific R. R. between Chowchilla and Madera.

[Latitude $37^{\circ} 04^{\prime} 38.657^{\prime \prime}$. Longitude $120^{\circ} 11^{\prime} 31.055^{\prime \prime}$.]

\begin{tabular}{|c|c|c|c|c|}
\hline To station- & Azimuth. & Back azimuth. & Distan & \\
\hline 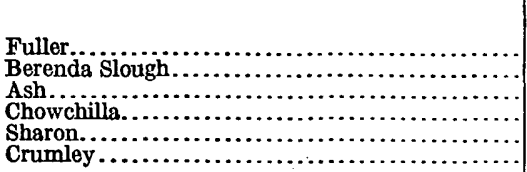 & $\begin{array}{rcc}\circ & \prime & \prime \prime \\
27 & 28 & 48.62 \\
61 & 23 & 15.80 \\
90 & 16 & 18.75 \\
131 & 29 & 42.34 \\
244 & 53 & 14.16 \\
341 & 27 & 19.25\end{array}$ & \begin{tabular}{rcc}
\multicolumn{1}{c}{} &, & $\prime \prime$ \\
207 & 27 & 01.59 \\
241 & 18 & 24.28 \\
270 & 09 & 33.15 \\
311 & 27 & 12.715 \\
64 & 55 & 26.89 \\
161 & 28 & 13.69
\end{tabular} & $\begin{array}{r}\text { Log. meters. } \\
\text { 3. } 9786933 \\
\text { 4. } 1343554 \\
\text { 4. } 2205790 \\
\text { 3. } 9124851 \\
\text { 3. } 7783343 \\
\text { 3. } 8465001\end{array}$ & $\begin{array}{r}\text { Miles. } \\
5.916 \\
8.467 \\
10.326 \\
5.080 \\
3.730 \\
4.364\end{array}$ \\
\hline
\end{tabular}


FAIRMONT, MERCED COUNTY, CALIF.

Tank house at the Miller \& Lux Fremont ranch. To reach ranch from Gustin go south 2 miles and east 6 miles.

Signal and station mark: Center of tank.

[Latitude $37^{\circ} 13^{\prime} 58.293^{\prime \prime}$. Longitude $120^{\circ} 52^{\prime} 28.063^{\prime}$.]

\begin{tabular}{|c|c|c|c|c|}
\hline To station- & Azimuth. & Back azimuth. & Distan & \\
\hline 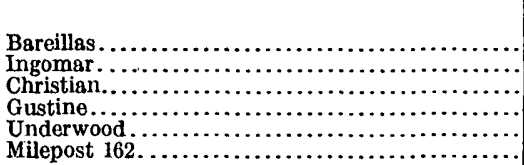 & \begin{tabular}{rcc}
\multicolumn{1}{c}{} & $\prime$ & $\prime \prime$ \\
32 & 29 & 59.59 \\
54 & 43 & 56.54 \\
77 & 23 & 45.43 \\
101 & 55 & 54.01 \\
324 & 50 & 18.74 \\
353 & 35 & 07.72
\end{tabular} & $\begin{array}{ccc}\circ & \prime & \prime \prime \\
212 & 24 & 02.62 \\
234 & 40 & 33.03 \\
257 & 15 & 47.61 \\
281,51 & 17.80 \\
144 & 51 & 36.50 \\
173 & 35 & 38.69\end{array}$ & $\begin{array}{r}\text { Log. meters. } \\
4.4346644 \\
4.0072640 \\
4.3002126 \\
4.0605287 \\
3.7408993 \\
4.0539241\end{array}$ & $\begin{array}{r}\text { Miles. } \\
16.905 \\
6.318 \\
12.407 \\
7.143 \\
3.422 \\
7.035\end{array}$ \\
\hline
\end{tabular}

FAIR OAKS, SAN LUIS OBISPO COUNTY, CALIF.

On highest point of a ridge about 8 miles a little north of west of Paso Robles, on the next place west of the San Ignacio ranch owned by Paderewski. Take the Paso Robles and Adelaida road for about 7 miles or until the summit is reached (elevation U. S. G. S., 1,940); at this point leave road and go south in pasture to highest point of ridge (about 0.5 mile in distance and 400 feet in elevation). All timber was cut from the point, and an oak tree was set in the ground for a signal.

Station mark: Tablet set in a rock placed 0.92 meter west of signal. Position of tablet was computed. Azimuth from tablet to signal, $274^{\circ} 50^{\prime}$; distance, 0.92 meter.

[Latitude $35^{\circ} 38^{\prime} 38.369^{\prime \prime}$. Longitude $120^{\circ} 47^{\prime} 36.689^{\prime \prime}$.]

\begin{tabular}{|c|c|c|c|c|}
\hline To station- & Azimuth. & Back azimuth. & Dista & \\
\hline 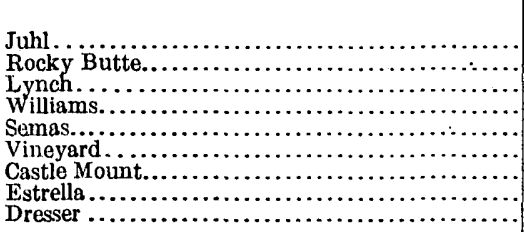 & $\begin{array}{rcc}\circ & \prime & \prime \prime \\
14 & 25 & 24.86 \\
95 & 45 & 52.62 \\
129 & 12 & 24.90 \\
151 & 17 & 43.97 \\
174 & 15 & 32.89 \\
214 & 41 & 01.53 \\
231 & 15 & 34.56 \\
248 & 07 & 48.99 \\
288 & 50 & 25.90\end{array}$ & \begin{tabular}{rcc}
\multicolumn{1}{c}{$\circ$} & $\prime$ & $\prime \prime$ \\
194 & 23 & 53.67 \\
275 & 36 & 35.73 \\
309 & 05 & 42.01 \\
331 & 10 & 27.19 \\
354 & 14 & 29.55 \\
34 & 45 & 14.03 \\
51 & 31 & 30.00 \\
68 & 17 & 22.55 \\
108 & 58 & 09.39
\end{tabular} & $\begin{array}{r}\text { Log. meters. } \\
\text { 4. } 2002766 \\
\text { 4. } 3829363 \\
4.3497869 \\
4.5906538 \\
4.4340556 \\
4.2808062 \\
4.7201779 \\
4.4252600 \\
4.3258150\end{array}$ & $\begin{array}{r}\text { Miles. } \\
9.854 \\
15.007 \\
13.904 \\
24.210 \\
16.881 \\
11.862 \\
32.623 \\
16.543 \\
13.157\end{array}$ \\
\hline
\end{tabular}

FAUCHER MONUMENT, MERCED COUNTY, CALIF.

A granite tombstone about 50 feet high, erected in memory of George Hicks Faucher, and inscribed "Born in New York State Feb. 9, 1828. Died in California, March 30, 1900," at a station on the Atchison, Topeka \& Santa Fe Ry. called Tuttle, about 5 miles east of Merced.

Signal and station mark: Center of monument.

[Latitude $37^{\circ} 17^{\prime} 44.646^{\prime \prime}$. Longitude $120^{\circ} 22^{\prime} 40.328^{\prime \prime}$.]

\begin{tabular}{|c|c|c|c|c|}
\hline To station- & Azimuth. & Back azimuth. & Distar & ce. \\
\hline 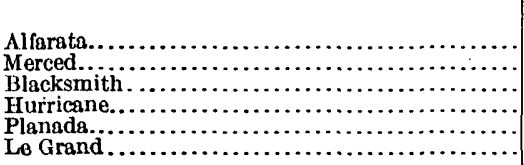 & \begin{tabular}{rcc}
\multicolumn{1}{c}{} & $\prime$ & $\prime \prime$ \\
68 & 47 & 44.74 \\
97 & 33 & 34.19 \\
177 & 06 & 32.73 \\
211 & 05 & 12.59 \\
278 & 10 & 42.96 \\
283 & 45 & 10.56
\end{tabular} & \begin{tabular}{rcc}
\multicolumn{1}{c}{$\circ$} & $\prime$ & $\prime \prime$ \\
248 & 45 & 33.64 \\
277 & 29 & 44.07 \\
357 & 06 & 17.29 \\
31 & 06 & 50.86 \\
98 & 12 & 41.59 \\
103 & 50 & 07.34
\end{tabular} & $\begin{array}{r}\text { Log. meters. } \\
\text { 3. } 7573088 \\
3.9746697 \\
4.0937954 \\
3.8879045 \\
3.6877652 \\
4.0943978\end{array}$ & $\begin{array}{r}\text { Miles. } \\
3.554 \\
5.862 \\
7.712 \\
4.800 \\
3.028 \\
7.722\end{array}$ \\
\hline
\end{tabular}


FIREBAUGH, FRESHO COUNTY, CALIF.

-Windmill without wheel at a hotel in Firebaugh owned by L. Pucciwelli. Signal: Mill frame on tank platform.

Station mark: Pipe in well under mill.

[Latitude $36^{\circ} 51^{\prime} 36.223^{\prime \prime}$. Longitude $120^{\circ} 27^{\prime} 26.707^{\prime \prime}$.]

\begin{tabular}{|c|c|c|c|c|}
\hline Tostation- & Aziminth. & Back azimuth. & Dista & \\
\hline 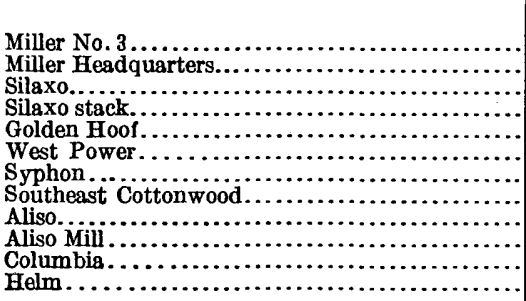 & $\begin{array}{rrr}\circ & \prime & \prime \prime \\
38 & 39 & 47.79 \\
58 & 23 & 55.72 \\
107 & 15 & 42.11 \\
126 & 57 & 48.06 \\
159 & 42 & 42.40 \\
176 & 49 & 09.48 \\
206 & 38 & 32.69 \\
244 & 19 & 48.74 \\
263 & 03 & 16.23 \\
269 & 21 & 52.57 \\
279 & 05 & 14.98 \\
313 & 41 & 39.36\end{array}$ & $\begin{array}{rcc}\circ & \prime & \prime \prime \\
218 & 36 & 06.91 \\
238 & 20 & 22.21 \\
287 & 13 & 06.31 \\
306 & 55 & 23.90 \\
339 & 41 & 08.11 \\
356 & 48 & 59.36 \\
26 & 41 & 36.55 \\
64 & 27 & 11.90 \\
83 & 08 & 23.29 \\
89 & 29 & 07.79 \\
99 & 08 & 49.02 \\
133 & 44 & 33.14\end{array}$ & $\begin{array}{r}\text { Log. meters. } \\
\text { 4. } 1654803 \\
4.0155529 \\
\text { 3. } 8282818 \\
\text { 3. } 8717451 \\
4.0488641 \\
\text { 3. } 8762201 \\
\text { 4. } 2272448 \\
\text { 4. } 3067411 \\
4.1061723 \\
\text { 4. } 2546146 \\
\text { 3. } 9520097 \\
3.9973857\end{array}$ & $\begin{array}{r}\text { Miles. } \\
9.096 \\
6.440 \\
4.184 \\
4.625 \\
6.954 \\
4.673 \\
10.486 \\
12.592 \\
7.935 \\
11.168 \\
5.564 \\
6.176\end{array}$ \\
\hline
\end{tabular}

FLAT TOP, MARIPOSA COUNTY, CALIF.

On a flat, rocky hill, 10 miles east of Le Grand, a station on the Atchison, Topeka \& Santa Fe Ry. Take road east from Le Grand 7 miles to ranch house on creek, turn in gate west of ranch house, thence follow left-hand road inside of gate to station.

Signal: Cairn 7 feet high.

Station mark: Tablet under center of cairn.

[Latitude $37^{\circ} 16^{\prime} 18.291^{\prime \prime}$. Longitude $120^{\circ} 06^{\prime} 37.152^{\prime \prime}$.]

\begin{tabular}{|c|c|c|c|c|}
\hline To station- & Azimuth. & Back azimuth. & Dist & nce. \\
\hline 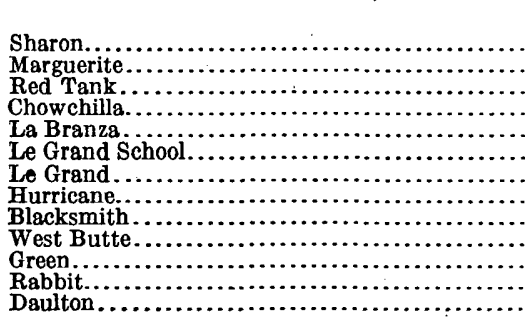 & \begin{tabular}{rcc}
\multicolumn{1}{c}{} & $\prime$ & $\prime \prime$ \\
5 & 28 & 20.74 \\
24 & 49 & 29.32 \\
21 & 51 & 49.23 \\
39 & 38 & 57.84 \\
60 & 33 & 03.79 \\
69 & 28 & 59.53 \\
88 & 33 & 37.80 \\
115 & 15 & 13.87 \\
121 & 49 & 44.89 \\
146 & 45 & 19.51 \\
273 & 33 & 47.77 \\
313 & 08 & 26.65 \\
323 & 47 & 50.27
\end{tabular} & $\begin{array}{rcc}\circ & \prime & \prime \prime \\
185 & 27 & 36.12 \\
204 & 47 & 10.49 \\
201 & 50 & 37.68 \\
219 & 32 & 30.19 \\
240 & 25 & 24.07 \\
249 & 23 & 50.78 \\
268 & 28 & 51.22 \\
295 & 07 & 08.26 \\
301 & 39 & 45.25 \\
326 & 40 & 51.91 \\
93 & 38 & 09.27 \\
113 & 14 & 57.74 \\
143 & 52 & 27.87\end{array}$ & \begin{tabular}{|r} 
Log. meters. \\
4.2812386 \\
4.1299824 \\
3.8937089 \\
4.3215165 \\
4.3330243 \\
4.1279427 \\
4.0668279 \\
4.3384349 \\
4.4566259 \\
4.2963446 \\
4.0277939 \\
4.3400157 \\
4.2830273
\end{tabular} & $\begin{array}{r}\text { Miles. } \\
11.874 \\
8.382 \\
4.865 \\
13.028 \\
13.377 \\
8.342 \\
7.247 \\
13.545 \\
17.782 \\
12.294 \\
6.624 \\
13.595 \\
11.923\end{array}$ \\
\hline
\end{tabular}

FOWLER, FRESNO COUNTY, CALIF. (Not occupied. No check.)

The high black steel tank in the town of Fowler.

Station mark: Center of tank.

[Latitude $36^{\circ} 37^{\prime} 48.607^{\prime \prime}$. Longitude $119^{\circ} 40^{\prime} 29.976^{\prime \prime}$.]

\begin{tabular}{|c|c|c|c|c|}
\hline To station- & Azimuth. & Back azimuth. & Distan & \\
\hline 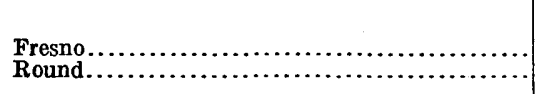 & $\begin{array}{ccc}\circ & , & \prime \prime \\
139 & 35 & 42.34 \\
215 & 07 & 40.97\end{array}$ & \begin{tabular}{rcc}
\multicolumn{1}{c}{} & $\prime$ & $\prime \prime$ \\
319 & 31 & 39.24 \\
35 & 13 & 37.15
\end{tabular} & \begin{tabular}{|c|} 
Log. meters. \\
4.1924664 \\
4.4091957
\end{tabular} & $\begin{array}{r}\text { Miles. } \\
9.679 \\
15.942\end{array}$ \\
\hline
\end{tabular}


FREMONT, SAN BENITO AND MONTEREY COUNTIES, CAIIF. (Not occupled.)

Highest point of Fremont Peak, a well-known mountain about 10 miles southwest of Hollister.

[Latitude $36^{\circ} 45^{\prime} 27.105^{\prime \prime}$. Longitude $121^{\circ} 30^{\prime} 12.293^{\prime \prime}$.]

\begin{tabular}{|c|c|c|c|c|}
\hline To station- & Azimuth. & Back azimuth. & Dista & \\
\hline 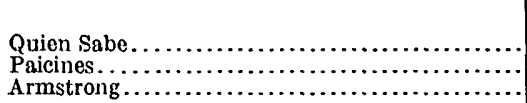 & $\begin{array}{ccc}\circ & \prime & \prime \prime \\
252 & 52 & 25.44 \\
270 & 44 & 50.36 \\
313 & 43 & 52.00\end{array}$ & $\begin{array}{rcc}\circ & \prime & \prime \prime \\
73 & 02 & 39.82 \\
90 & 51 & 55.13 \\
133 & 50 & 39.40\end{array}$ & \begin{tabular}{|c|} 
Log. meter8. \\
4.4248932 \\
4.2457276 \\
4.3702053
\end{tabular} & $\begin{array}{r}\text { Miles. } \\
16.529 \\
10.942 \\
14.573\end{array}$ \\
\hline
\end{tabular}

FRESNO COURTHOUSE, FRESNO COUNTY, CALIF.

Signal: Cupola on courthouse.

Station mark: Center of cupola on courthouse.

[Latitude $36^{\circ} 44^{\prime} 13.207^{\prime \prime}$. Longitude $119^{\circ} 47^{\prime} 16.907^{\prime \prime}$.]

\begin{tabular}{|c|c|c|c|c|}
\hline Tostation- & Azimuth. & Back azimuth. & Dista & \\
\hline 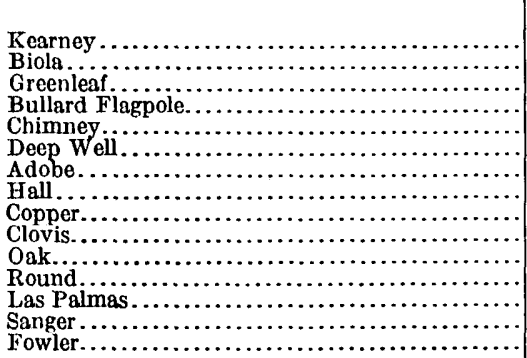 & $\begin{array}{rcc}\circ & \prime & \prime \prime \\
82 & 04 & 54.17 \\
109 & 16 & 39.85 \\
140 & 29 & 17.04 \\
162 & 09 & 36.12 \\
163 & 14 & 19.68 \\
164 & 05 & 22.21 \\
168 & 48 & 02.27 \\
187 & 26 & 22.98 \\
210 & 18 & 11.12 \\
219 & 31 & 15.51 \\
222 & 42 & 02.32 \\
249 & 46 & 51.75 \\
254 & 23 & 38.37 \\
278 & 31 & 54.90 \\
319 & 31 & 39.24\end{array}$ & \begin{tabular}{rcc}
\multicolumn{1}{c}{} & \multicolumn{1}{c}{$\prime \prime$} \\
262 & 00 & 21.89 \\
289 & 08 & 39.24 \\
320 & 23 & 57.78 \\
342 & 07 & 59.68 \\
343 & 13 & 24.90 \\
344 & 03 & 03.43 \\
348 & 45 & 23.68 \\
7 & 27 & 40.80 \\
30 & 23 & 18.70 \\
39 & 34 & 27.92 \\
42 & 51 & 06.41 \\
69 & 56 & 52.01 \\
74 & 26 & 52.16 \\
98 & 40 & 13.87 \\
139 & 35 & 42.34
\end{tabular} & $\begin{array}{r}\text { Log. meters. } \\
4.0697740 \\
4.3313870 \\
4.3168404 \\
4.1147057 \\
3.8957436 \\
4.3202756 \\
4.5266352 \\
4.3943012 \\
4.4007709 \\
4.0974228 \\
4.5198238 \\
4.4229203 \\
3.9212635 \\
4.3210465 \\
4.1924664\end{array}$ & $\begin{array}{r}\text { Miles. } \\
7.297 \\
13.327 \\
12.888 \\
8.092 \\
4.888 \\
12.990 \\
20.892 \\
15.405 \\
15.636 \\
7.776 \\
20.567 \\
16.454 \\
5.183 \\
13.014 \\
9.679\end{array}$ \\
\hline
\end{tabular}

FULLER, MADERA COUNTY, CALIF.

On the bank of Mr. Fuller's reservoir. It can be reached by following road due south from Chowchilla 9 miles to the Fuller ranch.

Signal: Lumber structure with cross targets.

Station mark: Iron bench-mark post centered under signal.

[Latitude $37^{\circ} 00^{\prime} 04.605^{\prime \prime}$. Longitude $120^{\circ} 14^{\prime} 28.744^{\prime \prime}$.]

\begin{tabular}{|c|c|c|c|c|}
\hline To station- & Azimuth. & Back azimuth. & Distar & \\
\hline 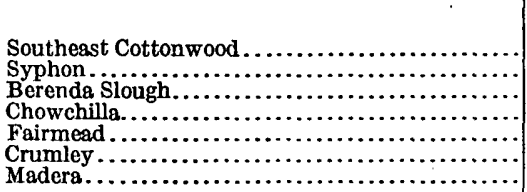 & \begin{tabular}{rcc}
\multicolumn{1}{c}{} & \multicolumn{1}{c}{} & $\prime \prime$ \\
8 & 04 & 38.18 \\
87 & 06 & 19.91 \\
104 & 13 & 09.51 \\
172 & 51 & 30.72 \\
207 & 27 & 01.59 \\
254 & 51 & 47.84 \\
285 & 49 & 32.04
\end{tabular} & \begin{tabular}{rcc}
\multicolumn{1}{c}{} & $\prime$ & $\prime \prime$ \\
188 & 04 & 14.33 \\
267 & 01 & 49.03 \\
284 & 10 & 05.20 \\
352 & 50 & 48.32 \\
27 & 28 & 48.62 \\
74 & 54 & 29.19 \\
105 & 56 & 02.64
\end{tabular} & \begin{tabular}{c|} 
Log. meters. \\
3.8442016 \\
4. 0674339 \\
3.8925604 \\
4.1452213 \\
3.9786933 \\
3.8365911 \\
4. 2226242
\end{tabular} & $\begin{array}{r}\text { Miles. } \\
\text { 4. } 341 \\
7.257 \\
4.852 \\
8.681 \\
5.916 \\
4.265 \\
10.375\end{array}$ \\
\hline
\end{tabular}

GALLAGHER, FRESNO COUNTY, CAIIF.

Station is a windmill on the north side of James Gallagher's tank house, 0.5 mile south of San Joaquin River and about 8 miles north-northwest of Kerman.

Signal: Windmill.

Station mark: Iron pipe in well under mill. 
[Latitude $36^{\circ} 48^{\prime} 30.178^{\prime \prime}$. Longitude $120^{\circ} 06^{\prime} 54.537^{\prime \prime}$.]

\begin{tabular}{|c|c|c|c|c|}
\hline To station- & Azimuth. & Back azimuth. & Dista & \\
\hline 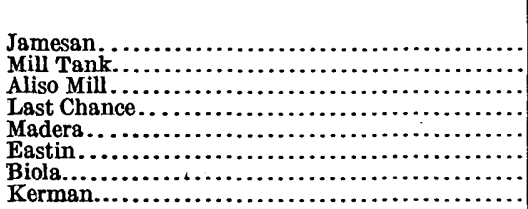 & 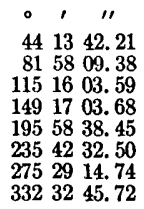 & \begin{tabular}{rcc}
\multicolumn{1}{c}{} & $\prime$ & $\prime \prime$ \\
224 & 10 & 29.16 \\
261 & 55 & 34.88 \\
295 & 10 & 59.87 \\
329 & 15 & 38.28 \\
16 & 00 & 35.41 \\
55 & 46 & 21.12 \\
95 & 32 & 50.98 \\
152 & 34 & 43.74
\end{tabular} & $\begin{array}{r}\text { Log. meters. } \\
4.0596341 \\
3.8100167 \\
4.1422972 \\
3.8394367 \\
\text { 4. } 2433982 \\
4.0581272 \\
\text { 3. } 9537156 \\
4.0258373\end{array}$ & $\begin{array}{r}\text { Miles. } \\
7.128 \\
4.012 \\
8.623 \\
4.293 \\
10.883 \\
7.104 \\
5.586 \\
6.595\end{array}$ \\
\hline
\end{tabular}

GOLDEN HOOF, FRESNO COUNTY, CALIF.

Station is windmill on west side of tank house at the Miller \& Lux Golden Hoof ranch, 7 miles southeast of Dos Palos.

Signal: Windmill.

Station mark: Center of well under mill.

[Latitude $36^{\circ} 57^{\prime} 16.708^{\prime \prime}$. Longitude $120^{\circ} 30^{\prime} 03.550^{\prime \prime}$.]

\begin{tabular}{|c|c|c|c|c|}
\hline To station- & Arimuth. & Back azimuth. & Dis & nce. \\
\hline 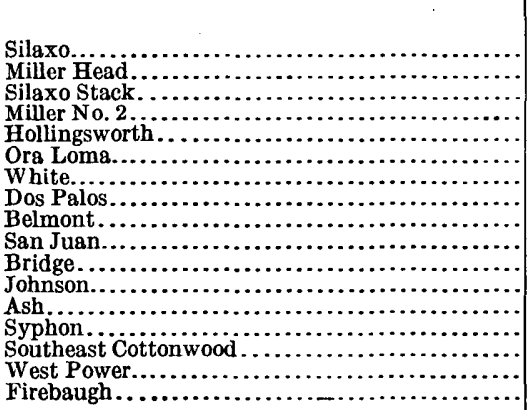 & $\begin{array}{rcc}\circ & \prime & \prime \prime \\
16 & 40 & 44.61 \\
17 & 13 & 45.61 \\
18 & 55 & 04.61 \\
46 & 00 & 16.75 \\
55 & 26 & 25.97 \\
66 & 05 & 44.56 \\
81 & 31 & 10.80 \\
108 & 09 & 08.30 \\
130 & 11 & 28.83 \\
135 & 20 & 48.85 \\
159 & 18 & 47.73 \\
174 & 33 & 45.71 \\
218 & 23 & 50.80 \\
248 & 08 & 20.86 \\
274 & 24 & 37.50 \\
310 & 45 & 46.99 \\
339 & 41 & 08.11\end{array}$ & \begin{tabular}{rcc}
\multicolumn{1}{c}{} &, & $\prime \prime$ \\
196 & 39 & 42.84 \\
197 & 11 & 45.99 \\
198 & 54 & 14.51 \\
225 & 55 & 03.54 \\
235 & 21 & 36.88 \\
245 & 58 & 15.37 \\
261 & 18 & 55.45 \\
288 & 04 & 39.19 \\
310 & 07 & 04.05 \\
315 & 15 & 11.46 \\
339 & 17 & 04.66 \\
354 & 32 & 17.49 \\
38 & 28 & 15.43 \\
68 & 12 & 59.26 \\
94 & 33 & 35.42 \\
130 & 47 & 11.12 \\
159 & 42 & 42.30
\end{tabular} & $\begin{array}{r}\text { Log. meters. } \\
3.9480607 \\
4.2221530 \\
3.8038730 \\
4.2544780 \\
4.1605565 \\
4.3065781 \\
4.4861133 \\
4.0661607 \\
4.1533735 \\
4.2943323 \\
4.0783409 \\
4.0871288 \\
4.2425475 \\
4.0910395 \\
4.3465805 \\
3.6603027 \\
4.0488641\end{array}$ & $\begin{array}{r}\text { Miles. } \\
5.513 \\
10.363 \\
3.956 \\
11.164 \\
8.993 \\
12.587 \\
19.031 \\
7.236 \\
8.845 \\
12.237 \\
7.442 \\
7.594 \\
10.862 \\
7.663 \\
13.802 \\
2.842 \\
6.954\end{array}$ \\
\hline
\end{tabular}

GRANVILIE, FRESNO COUNTY, CALIF. (Not occupied.)

Granville School, 4 miles northwest of Sanger.

Signal and station mark: Center of cupola.

[Latitude $36^{\circ} 44^{\prime}$ 08.493"'. Longitude $119^{\circ} 34^{\prime} 58.488^{\prime \prime}$.]

\begin{tabular}{|c|c|c|c|c|}
\hline To station- & Azimuth. & Back azimuth. & Distar & \\
\hline 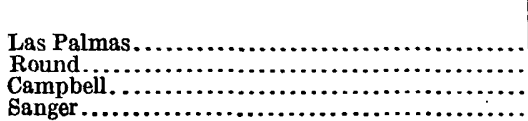 & $\begin{array}{ccc}\circ & \prime & \prime \prime \\
103 & 05 & 45.01 \\
215 & 15 & 04.46 \\
286 & 57 & 25.32 \\
321 & 24 & 06.59\end{array}$ & \begin{tabular}{rcc}
\multicolumn{1}{c}{} & $\prime$ & $\prime \prime$ \\
283 & 01 & 37.02 \\
35 & 17 & 42.61 \\
107 & 03 & 13.71 \\
141 & 25 & 04.02
\end{tabular} & \begin{tabular}{|c|} 
Log. meters. \\
4.0235123 \\
4.0547474 \\
4.1796546 \\
3.5822222
\end{tabular} & $\begin{array}{r}\text { Miles. } \\
6.559 \\
7.049 \\
9.397 \\
2.375\end{array}$ \\
\hline
\end{tabular}

GRAVES, MONTEREY COUNTY, CAIIF. (Not occupied.)

Large pine tree near the summit of Bald Hill, owned by Mr. Graves. About 5 miles south of Lockwood.

Station mark: Center of tree.

[Latitude $35^{\circ} 51^{\prime} 50.857^{\prime \prime}$. Longitude $121^{\circ} 07^{\prime} 25.449^{\prime \prime}$.]

\begin{tabular}{|c|c|c|c|c|}
\hline To station- & Azimuth. & Back azimuth. & Distar & \\
\hline 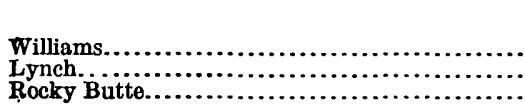 & $\begin{array}{ccc}\bullet & \prime & \prime \prime \\
228 & 41 & 05.58 \\
309 & 24 & 31.63 \\
345 & 04 & 45.77\end{array}$ & $\begin{array}{ccc}\circ & \prime \prime & \prime \prime \\
48 & 45 & 24.83 \\
129 & 29 & 23.34 \\
165 & 07 & 02.18\end{array}$ & \begin{tabular}{|r} 
Log. meters. \\
4.1687794 \\
4.2096584 \\
4.3578927
\end{tabular} & $\begin{array}{r}\text { Miles. } \\
9.165 \\
10.069 \\
14.166\end{array}$ \\
\hline
\end{tabular}


GREEN, MARIPOSA COUNTY, CALIF.

On a bald hill, locally known as Green Mountain. The Green Mountain copper mine is just under the hill on the south side. The road from Raymond to Mariposa passes within a mile of the hill at Seaton's saloon, 8 miles from Raymond. Animals can be ridden to the point.

Signal: Cairn 8 feet high.

Station mark: Triangulation tablet; azimuth to signal, $146^{\circ}$; distance, 1.10 meters. Latitude $37^{\circ} 15^{\prime} 56.92^{\prime \prime}(1908), 37^{\circ} 15^{\prime} 56.579^{\prime \prime}(1915)$. Longitude $119^{\circ} 59^{\prime} 24.51^{\prime \prime}(1908), 119^{\circ} 59^{\prime} 25.318^{\prime \prime}(1915)$.

\begin{tabular}{|c|c|c|c|c|}
\hline To station- & Azimuth. & Back azimuth. & \multicolumn{2}{|c|}{ Distance. } \\
\hline . & 0,11 & 0111 & Log. meters. & Miles. \\
\hline Bullion (1908) ....... & 1732150.63 & 3532024.66 & 4. 4782044 & $\ldots \ldots \ldots$ \\
\hline Moore Hill (1908).... & 1812124.88 & 12133.01 & 4. 1437321 & ......... \\
\hline Devil (1908) ....... & $\begin{array}{lll}217 & 03 & 19.18\end{array}$ & 371226.66 & 4. 5650238 & $\cdots \cdots$ \\
\hline ind $(1908) \ldots$ & 2335748.00 & $\begin{array}{lll}54 & 13 & 23.09\end{array}$ & 4. 6699597 & ... \\
\hline Doadwood (1908).... & $258 \quad 4608.37$ & 785713.77 & 4. 4404747 & $\cdots$ \\
\hline$(1908) \ldots \ldots$ & 3355445.56 & 1555926.04 & 4. 4490909 & $\ldots$ \\
\hline Rabbit (1908). . & 3393828.41 & 1594038.41 & 4. 1833582 & $\ldots \ldots$ \\
\hline Sharon $(1915) \ldots$ & 341430.93 & 2140925.33 & 4. 3461516 & 13.78 \\
\hline Marguerite (1915).. & 544114.52 & 2343434.53 & 4. 3009780 & 12.426 \\
\hline Red Tank (1915). & $\begin{array}{llll}64 & 06 & 03.16\end{array}$ & 2440030.31 & 4. 1783613 & 9. 369 \\
\hline Le Grand (1915)... & 910119.60 & 2705211.55 & 4. 3483662 & 13.858 \\
\hline Flat Top (1915)..... & 933809.27 & 2733347.77 & 4. 0277939 & 6.624 \\
\hline Jest Butte (1915)......... & 1284555.93 & 3083706.40 & 4. 4397964 & 17. 106 \\
\hline bit $(1915) \ldots \ldots \ldots \ldots$ & 3393857.68 & 1594107.65 & 4. 1834615 & 9.480 \\
\hline & 3572344.70 & 1772401.20 & 4.1713441 & \\
\hline
\end{tabular}

GREENLEAF, MADERA COUNTY, CALIF.

A red tank, belonging to the railroad company, at Greenleaf, a station on the Atchison, Topeka \& Santa Fe Ry., 8 miles southeast of Madera.

Signal and station mark: Center of tank.

[Latitude $36^{\circ} 52^{\prime} 51.990^{\prime \prime}$. Longitude $119^{\circ} 56^{\prime} 09.788^{\prime \prime}$.]

\begin{tabular}{|c|c|c|c|c|}
\hline To station- & Aximuth. & Back azimuth. & Distar & \\
\hline 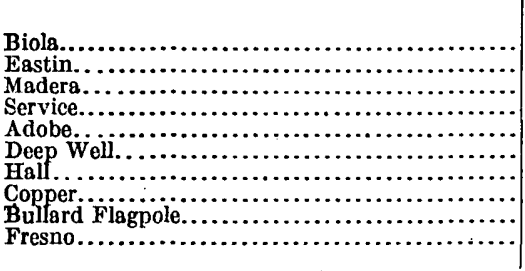 & \begin{tabular}{rcc}
\multicolumn{1}{c}{} & $\prime$ & $\prime \prime$ \\
38 & 13 & 39.73 \\
75 & 57 & 12.56 \\
128 & 14 & 55.34 \\
187 & 57 & 38.84 \\
201 & 20 & 41.41 \\
241 & 06 & 56.07 \\
242 & 18 & 04.46 \\
257 & 26 & 49.03 \\
291 & 17 & 44.83 \\
320 & 23 & 57.78
\end{tabular} & \begin{tabular}{rcc}
\multicolumn{1}{c}{} & $\prime$ & $\prime \prime$ \\
218 & 10 & 49.56 \\
255 & 54 & 34.49 \\
308 & 10 & 25.10 \\
7 & 58 & 11.29 \\
21 & 23 & 22.94 \\
61 & 09 & 57.00 \\
62 & 24 & 42.50 \\
77 & 37 & 17.12 \\
111 & 21 & 27.91 \\
140 & 29 & 17.04
\end{tabular} & \begin{tabular}{r|} 
Log. meters. \\
4.0557672 \\
3.8277556 \\
4.1514200 \\
3.9844702 \\
4.2610799 \\
3.9303794 \\
4.2675243 \\
4.4235469 \\
3.9950910 \\
4.3168404
\end{tabular} & $\begin{array}{r}\text { Miles. } \\
\text { 7. } 065 \\
4.179 \\
8.886 \\
5.995 \\
11.335 \\
5.293 \\
11.505 \\
16.478 \\
6.144 \\
12.888\end{array}$ \\
\hline
\end{tabular}

GRUBER, FRESNO COUNTY, CALIF. (Not occupied.)

Station is windmill at Mr. Gruber's ranch, about 8 miles west of Mendota, a small town on the Southern Pacific R. R.

[Latitude $36^{\circ} 45^{\prime} 47.136^{\prime \prime}$. Longitude $120^{\circ} 37^{\prime} 50.062^{\prime \prime}$.]

\begin{tabular}{|c|c|c|c|c|}
\hline Tostation- & Azimuth. & Back azimuth. & Dist & nce. \\
\hline 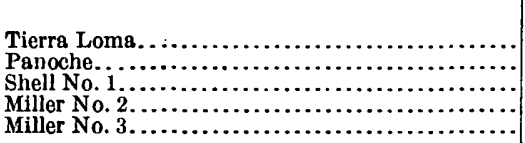 & $\begin{array}{rcc} & & \prime \prime \\
31 & 44 & 36.66 \\
70 & 54 & 13.27 \\
103 & 43 & 06.23 \\
171 & 08 & 23.60 \\
276 & 04 & 07.38\end{array}$ & \begin{tabular}{rcc}
\multicolumn{1}{c}{} & $\prime$ & $\prime \prime$ \\
211 & 43 & 38.63 \\
250 & 49 & 26.18 \\
283 & 41 & 06.99 \\
351 & 07 & 50.56 \\
96 & 06 & 39.79
\end{tabular} & \begin{tabular}{r|} 
Log. meters. \\
3. 6604027 \\
4. 1004317 \\
3. 7062818 \\
3. 9480559 \\
3.8029572
\end{tabular} & $\begin{array}{r}\text { Miles. } \\
2.843 \\
7.830 \\
3.160 \\
5.513 \\
3.947\end{array}$ \\
\hline
\end{tabular}


GUSTINE, MERCED COUNTY, CALIF.

The cupola of Catholic Church in Gustine.

Signal: Center of cupola.

Station mark: Center of cupola.

[Latitude $37^{\circ} 15^{\prime} 15.143^{\prime \prime}$. Longitude $121^{\circ} 00^{\prime} 04.458^{\prime \prime}$.]

\begin{tabular}{|c|c|c|c|c|}
\hline To station- & Azimuth. & Back azimuth. & Distar & \\
\hline 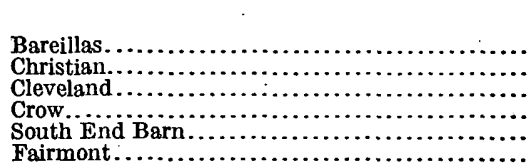 & $\begin{array}{rcc}\bullet & \prime & \prime \prime \\
7 & 30 & 19.34 \\
50 & 39 & 47.69 \\
98 & 25 & 05.77 \\
131 & 31 & 16.64 \\
228 & 08 & 37.94 \\
281 & 51 & 17.80\end{array}$ & \begin{tabular}{rcc}
\multicolumn{1}{c}{} & $\prime$ & $\prime \prime$ \\
187 & 28 & 57.84 \\
230 & 36 & 25.84 \\
278 & 20 & 23.36 \\
311 & 25 & 58.53 \\
48 & 13 & 19.20 \\
101 & 55 & 54.01
\end{tabular} & $\begin{array}{r}\text { Log. meters. } \\
4.4073108 \\
4.0269572 \\
4.0651103 \\
4.2368543 \\
4.1906513 \\
4.0605287\end{array}$ & $\begin{array}{r}\text { Miles. } \\
15.873 \\
6.612 \\
7.219 \\
10.720 \\
9.639 \\
7.143\end{array}$ \\
\hline
\end{tabular}

HALL, MADERA COUNTY, CALIF.

On top of a hill on the west bank of San Joaquin River, about 2 miles southwest of Friant. The Madera-Friant road passes the north end of the hill about 0.5 mile from signal.

Position of signal computed.

Station mark and signal: Timber with black and white cross targets.

Reference mark: Aluminum tablet. Azimuth from tablet to signal, $114^{\circ} 08^{\prime}$; distance, 4.8 feet.

[Latitude $36^{\circ} 57 \quad 0.666^{\prime \prime}$. Longitude $119^{\circ} 45^{\prime} 07.153^{\prime \prime}$.]

\begin{tabular}{|c|c|c|c|c|}
\hline To station- & Azimuth. & Back azimuth. & Distar & \\
\hline 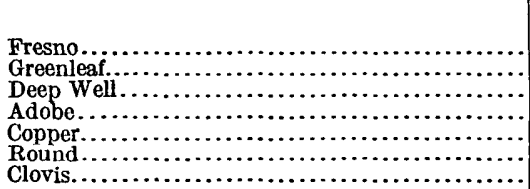 & $\begin{array}{rcc}\circ & \prime & \prime \prime \\
7 & 27 & 40.80 \\
62 & 24 & 42.50 \\
63 & 25 & 18.76 \\
130 & 47 & 43.97 \\
286 & 47 & 00.35 \\
305 & 30 & 54.68 \\
342 & 21 & 30.56\end{array}$ & $\begin{array}{ccc}\circ & \prime & \prime \prime \\
187 & 26 & 22.98 \\
242 & 18 & 04.46 \\
243 & 21 & 41.64 \\
310 & 43 & 46.88 \\
106 & 50 & 50.73 \\
125 & 39 & 38.61 \\
162 & 23 & 25.57\end{array}$ & $\begin{array}{r}\text { Log. meters. } \\
4.3943012 \\
4.2675243 \\
3.9999819 \\
4.1093124 \\
3.9959738 \\
4.4245034 \\
4.1950005\end{array}$ & $\begin{array}{r}\text { Miles. } \\
15.405 \\
11.505 \\
6.213 \\
7.992 \\
6.156 \\
16.514 \\
9.735\end{array}$ \\
\hline
\end{tabular}

HARRIS, SAN BENITO COUNTY, CALIF.

On a brushy mountain about 12 miles from San Benito and about 1 mile south of Mr. Harris's ranch. From San Benito take the Hollister road through Bear Valley to Mr. Henry Melendy's ranch, thence go through a gate on the Salinas Valley road to Mr. Harris's ranch. This is as far as a wagon can be taken. From Mr. Harris's ranch go south up ridge to the highest point. Animals can be ridden all the way up.

Signal: A pine tree set 3 feet in the ground.

Station mark: A tablet set in rock placed in ground. Azimuth from tablet to signal, $87^{\circ} 03^{\prime}$; distance, 1.10 meters.

[Latitude $36^{\circ} 33^{\prime} 08.688^{\prime \prime}$. Longitude $121^{\circ} 13^{\prime} 15.137^{\prime \prime}$.]

\begin{tabular}{|c|c|c|c|c|}
\hline To station- & Azimuth. & Back azimuth. & Distan & \\
\hline 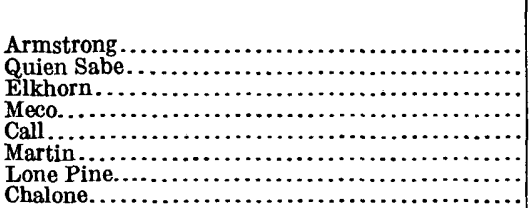 & $\begin{array}{ccc}\circ & \prime & \prime \prime \\
128 & 07 & 01.67 \\
180 & 24 & 06.89 \\
204 & 07 & 37.81 \\
213 & 18 & 21.43 \\
245 & 36 & 43.70 \\
304 & 03 & 17.92 \\
306 & 10 & 21.70 \\
348 & 39 & 05.71\end{array}$ & \begin{tabular}{rcc}
\multicolumn{1}{c}{0} & $\prime$ & $\prime \prime$ \\
308 & 03 & 41.89 \\
0 & 24 & 12.06 \\
24 & 10 & 25.50 \\
33 & 24 & 13.23 \\
65 & 42 & 21.44 \\
124 & 07 & 37.33 \\
126 & 12 & 14.29 \\
168 & 40 & 01.22
\end{tabular} & \begin{tabular}{|c|} 
Log. meters. \\
4.0248336 \\
4.4851199 \\
4.2322725 \\
4.4252340 \\
4.1893173 \\
4. 1171623 \\
3. 7656121 \\
4.0722502
\end{tabular} & $\begin{array}{r}\text { Miles. } \\
\mathbf{6 . 5 7 9} \\
18.988 \\
10.608 \\
16.542 \\
\mathbf{9 . 6 0 9} \\
\mathbf{8 . 1 3 8} \\
\mathbf{3 . 6 2 2} \\
\mathbf{7 . 3 3 8}\end{array}$ \\
\hline
\end{tabular}




\section{HELLMAN, SAN LUIS OBISPO COUNTY, CALIF}

On a low hill about 4.5 miles northwest of San Miguel. The road to the Hellman ranch passes under the foot of the hill, and a buckboard was driven to the summit, which is the flat top of a ridge. There are oak trees on the ridge but none within 100 yards of the signal.

Signal: A redwood post 4 by 4 inches and 16 feet long, set about 4 feet in the ground, with two white targets 3 feet square at right angles to each other on it.

Station mark: A triangulation tablet set in a rock placed in the ground 1.34 meters. a little east of north of signal. The position of the tablet was determined. Azimuth from tablet to signal, $18^{\circ} 22^{\prime}$; distance, 1.34 meters.

[Latitude $35^{\circ} 46^{\prime} 41.123^{\prime \prime}$. Longitude $120^{\circ} 46^{\prime} 06.249^{\prime \prime}$.]

\begin{tabular}{|c|c|c|c|c|}
\hline To station- & Azimuth. & Back azimuth. & Dista & \\
\hline 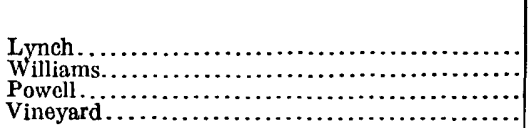 & \begin{tabular}{rcc}
\multicolumn{1}{c}{} & $\prime$ & $\prime \prime$ \\
87 & 52 & 15.54 \\
132 & 36 & 50.54 \\
190 & 33 & 50.48 \\
264 & 34 & 04.51
\end{tabular} & \begin{tabular}{rcc}
\multicolumn{1}{c}{0} & \multicolumn{1}{c}{$\prime$} \\
267 & 44 & 39.13 \\
312 & 28 & 40.07 \\
10 & 35 & 39.07 \\
84 & 37 & 24.55
\end{tabular} & $\begin{array}{c}\text { Log. meters. } \\
\text { 4. } 2927839 \\
4.4549712 \\
\text { 4. } 4031826 \\
3.9360395\end{array}$ & $\begin{array}{r}\text { Miles. } \\
12.194 \\
17.714 \\
15.723 \\
5.363\end{array}$ \\
\hline
\end{tabular}

HELM, FRESNO COUNTY, CALIF.

Tank house at the Miller \& Lux Helm ranch, 4 miles north of Mendota.

Signal: Center of tank house.

Station mark: Center of concrete floor of tank house.

[Latitude $36^{\circ} 47^{\prime} 53.367^{\prime \prime}$. Longitude $120^{\circ} 22^{\prime} 36.808^{\prime \prime}$.]

\begin{tabular}{|c|c|c|c|c|}
\hline To station- & Azimuth. & Back azimuth. & Distar & \\
\hline 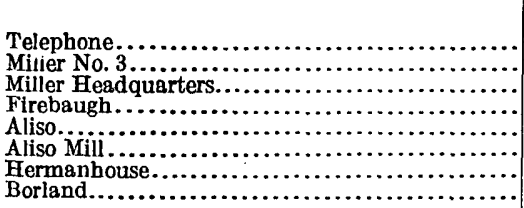 & \begin{tabular}{rcc}
\multicolumn{1}{c}{} & $\prime$ & $\prime \prime$ \\
46 & 13 & 39.50 \\
74 & 26 & 16.42 \\
95 & 10 & 13.86 \\
133 & 44 & 33.14 \\
213 & 10 & 34.66 \\
236 & 48 & 58.18 \\
316 & 48 & 36.08 \\
341 & 50 & 55.70
\end{tabular} & \begin{tabular}{rcc}
\multicolumn{1}{c}{.} & $\prime$ & $\prime \prime$ \\
226 & 08 & 43.25 \\
254 & 19 & 42.12 \\
275 & 03 & 46.83 \\
313 & 41 & 39.36 \\
33 & 12 & 47.70 \\
56 & 53 & 19.31 \\
136 & 50 & 47.79 \\
161 & 52 & 40.46
\end{tabular} & \begin{tabular}{|c|} 
Log. meters. \\
4.2310930 \\
4.2293593 \\
4.2062559 \\
3.9973857 \\
4.0018767 \\
4.1103638 \\
3.9016663 \\
4.1447886
\end{tabular} & $\begin{array}{r}\text { Miles. } \\
10.579 \\
10.537 \\
9.991 \\
6.176 \\
6.241 \\
8.012 \\
4.955 \\
8.672\end{array}$ \\
\hline
\end{tabular}

HERMANHOUSE, FRESNO COUNTY, CALIF.

Tank house on the Hermanhouse ranch, 6 miles more or less east of Mendota and 0.5 mile north of the Whites Bridge-Fresno road.

Signal and station mark: Center of tank house.

[Latitude $36^{\circ} 44^{\prime} 44.711^{\prime \prime}$. Longitude $120^{\circ} 18^{\prime} 56.822^{\prime \prime}$.]

\begin{tabular}{|c|c|c|c|c|}
\hline To station- & Azimuth. & Back azimuth. & Distar & ce. \\
\hline 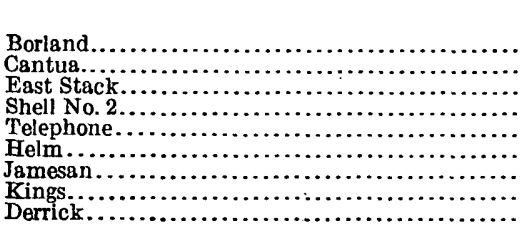 & \begin{tabular}{rcc}
\multicolumn{1}{c}{ ' } & \multicolumn{1}{c}{} & $\prime \prime$ \\
8 & 30 & 31.01 \\
11 & 35 & 29.43 \\
26 & 44 & 28.41 \\
46 & 35 & 53.96 \\
71 & 27 & 51.79 \\
136 & 50 & 47.79 \\
277 & 17 & 11.43 \\
310 & 51 & 51.34 \\
313 & 07 & 30.17
\end{tabular} & $\begin{array}{rcc}\circ & \prime & \prime \prime \\
188 & 30 & 04.18 \\
191 & 33 & 22.06 \\
206 & 41 & 15.81 \\
226 & 28 & 37.35 \\
251 & 20 & 44.18 \\
316 & 48 & 36.08 \\
97 & 21 & 10.56 \\
130 & 55 & 02.44 \\
133 & 13 & 52.11\end{array}$ & \begin{tabular}{r|} 
Log. meters. \\
3.8768482 \\
4.4221967 \\
4.2506831 \\
4.3981829 \\
4.2724558 \\
3.9016663 \\
4.0000079 \\
4.0209628 \\
4.3378948
\end{tabular} & $\begin{array}{r}\text { Miles. } \\
4.679 \\
16.427 \\
11.067 \\
15.543 \\
11.636 \\
4.955 \\
6.214 \\
6.521 \\
13.528\end{array}$ \\
\hline
\end{tabular}


HERIDON, FRESNO COUNTY, CALIF.

Station mark: Center of cupola on schoolhouse at Herndon, a small town on the Southern Pacific R. R. between Madera and Fresno. Later destroyed.

[Latitude $36^{\circ} 50^{\prime} 18.135^{\prime \prime}$. Longitude $120^{\circ} 54^{\prime} 53.225^{\prime \prime}$.]

\begin{tabular}{|c|c|c|c|c|}
\hline Tostation- & Azimuth. & Back azimuth. & Distar & \\
\hline 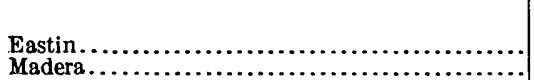 & $\begin{array}{ccc}\circ & \prime & \prime \prime \\
110 & 16 & 50.16 \\
136 & 04 & 07.65\end{array}$ & $\begin{array}{ccc}\circ & \prime & \prime \prime \\
290 & 13 & 26.25 \\
315 & 58 & 51.57\end{array}$ & $\begin{array}{r}\text { Log. meters. } \\
3.9531716 \\
4.2734974\end{array}$ & $\begin{array}{r}\text { Miles. } \\
5.579 \\
11.664\end{array}$ \\
\hline
\end{tabular}

HIGHLAND, MERCED COUNTY, CALIF.

On a small knoll 0.5 mile north of the Miller \& Lux Highland ranch and 2.5 miles northeast of the Miller \& Lux San Luis ranch; it is 0.25 mile southeast of willow tree, 1 mile west of San Joaquin River, and 0.5 mile north of slough. It can be reached by north and south road from Los Banos to Standard Oil pumping station 3 miles north of Los Banos, thence to San Luis ranch.

Signal: Quadripod with cross targets and flag.

Station mark: Iron bench-mark post centered under signal.

[Latitude $37^{\circ} 09^{\prime} 49.248^{\prime \prime}$. Longitude $120^{\circ} 45^{\prime} 58.227^{\prime \prime}$.]

\begin{tabular}{|c|c|c|c|c|}
\hline To station- & Azimuth. & Back azimuth. & Dist & nce. \\
\hline 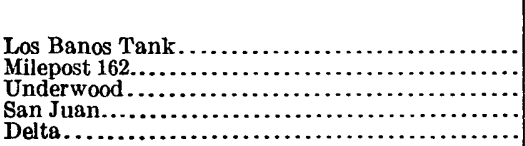 & $\begin{array}{rcc}\circ & \prime & \prime \prime \\
31 & 39 & 32.93 \\
66 & 52 & 18.43 \\
116 & 14 & 52.28 \\
313 & 21 & 03.91 \\
334 & 56 & 43.03\end{array}$ & $\begin{array}{ccc}\circ & \prime \prime \\
211 & 36 & 41.22 \\
246 & 48 & 53.96 \\
296 & 12 & 14.62 \\
133 & 25 & 01.88 \\
154 & 57 & 41.65\end{array}$ & \begin{tabular}{|c|} 
Log. meters. \\
4. 1269623 \\
3.9584686 \\
3.8563848 \\
4.1268982 \\
3.7527904
\end{tabular} & $\begin{array}{r}\text { Miles. } \\
8.324 \\
5.647 \\
4.464 \\
8.322 \\
3.517\end{array}$ \\
\hline
\end{tabular}

HOLLINGSWORTH, FRESNO COUNTY, CALIF.

Center of tank over a well 1,000 feet deep on the Hollingsworth tract, 9 miles south of South Dos Palos. It is about 1 mile east of road leading south from South Dos Palos.

Signal: Center of tank.

Station mark: Center of cement foundation and floor under tank.

[Latitude $36^{\circ} 5250.104^{\prime \prime}$. Longitude $120^{\circ} 38^{\prime} 04.839^{\prime \prime}$.]

\begin{tabular}{|c|c|c|c|c|}
\hline To station- & Azimuth. & Back azimuth. & Distar & \\
\hline 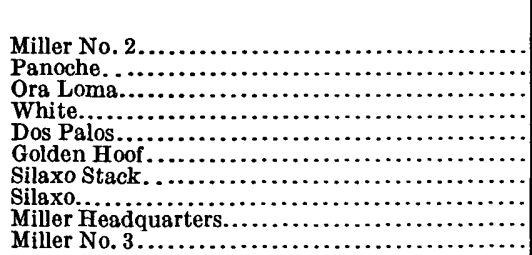 & \begin{tabular}{rcc}
\multicolumn{1}{c}{} & \multicolumn{1}{c}{$\prime \prime$} \\
13 & 10 & 56.85 \\
33 & 55 & 19.66 \\
89 & 55 & 55.72 \\
101 & 17 & 06.20 \\
184 & 02 & 28.04 \\
235 & 21 & 36.88 \\
257 & 24 & 12.73 \\
271 & 41 & 21.47 \\
317 & 48 & 21.59 \\
334 & 00 & 35.85
\end{tabular} & \begin{tabular}{rcc}
\multicolumn{1}{c}{} & $\prime$ & $\prime \prime$ \\
193 & 10 & 32.62 \\
213 & 50 & 41.02 \\
269 & 53 & 18.76 \\
281 & 09 & 40.43 \\
4 & 02 & 48.36 \\
55 & 26 & 25.97 \\
77 & 28 & 11.58 \\
91 & 45 & 08.58 \\
137 & 51 & 10.68 \\
154 & 03 & 17.32
\end{tabular} & $\begin{array}{r}\text { Log. meters. } \\
3.6421701 \\
4.3155823 \\
3.8195797 \\
4.2727986 \\
4.0744041 \\
4.1605565 \\
4.0041040 \\
3.9720590 \\
4.0173219 \\
4.1833237\end{array}$ & $\begin{array}{r}\text { Miles. } \\
2.726 \\
12.851 \\
4.101 \\
11.645 \\
7.375 \\
8.993 \\
6.273 \\
5.827 \\
6.467 \\
9.477\end{array}$ \\
\hline
\end{tabular}


HOLLISTER, SAN BENITO COUNTY, CALIF.

Cupola on courthouse at Hollister, the county seat of San Benito County, Calif. Station mark: Center of cupola.

[Latitude $36^{\circ} 51^{\prime} 08.514^{\prime \prime}$. Longitude $121^{\circ} 24^{\prime} 12.466^{\prime \prime}$.]

\begin{tabular}{|c|c|c|c|c|}
\hline To station- & Azimuth. & Back azimuth. & Distar & \\
\hline 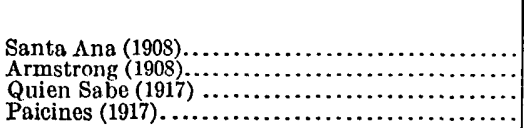 & $\begin{array}{ccc}\circ & \prime & \prime \prime \\
248 & 49 & 22.53 \\
313 & 20 & 25.50 \\
279 & 20 & 12.24 \\
321 & 07 & 24.50\end{array}$ & \begin{tabular}{rcc}
\multicolumn{1}{c}{$\circ$} & $\prime$ & $\prime \prime$ \\
68 & 55 & 31.44 \\
163 & 23 & 38.15 \\
99 & 26 & 51.55 \\
141 & 10 & 54.17
\end{tabular} & $\begin{array}{r}\text { Log. meters. } \\
4.2127407 \\
4.4459584 \\
4.2233829 \\
4.1408906\end{array}$ & $\begin{array}{r}\text { Miles. } \\
10.141 \\
17.35 \mathrm{C} \\
10.395 \\
8.595\end{array}$ \\
\hline
\end{tabular}

HONN, MERCED COUNTY, CALIF.

Tank house on Mr. Honn's ranch, about 12 miles south of Merced. Take Santa Rita road for 10 miles, thence go east 2 miles. It can be occupied.

Signal and station mark: Center of 40 -foot tank house.

[Latitude $37^{\circ} 08^{\prime} 28.870^{\prime \prime}$. Longitude $120^{\circ} 27^{\prime} 17.477^{\prime \prime}$.]

\begin{tabular}{|c|c|c|c|c|}
\hline To station- & Azimuth. & Back azimuth. & Dista & \\
\hline 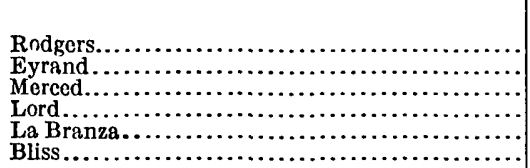 & $\begin{array}{ccc}\circ & \prime & \prime \prime \\
76 & 11 & 06.57 \\
137 & 36 & 31.72 \\
172 & 10 & 09.96 \\
189 & 04 & 24.74 \\
251 & 53 & 50.54 \\
297 & 54 & 01.94\end{array}$ & \begin{tabular}{rcc}
\multicolumn{1}{c}{} & $\prime$ & $\prime \prime$ \\
256 & 08 & 53.25 \\
317 & 32 & 57.40 \\
352 & 09 & 07.90 \\
9 & 04 & 44.11 \\
71 & 58 & 40.69 \\
117 & 55 & 48.79
\end{tabular} & \begin{tabular}{|r|} 
Log. meters. \\
3.7897568 \\
4. 1128238 \\
4.2681636 \\
3.7004943 \\
4.0957696 \\
3.6941369
\end{tabular} & $\begin{array}{r}\text { Miles. } \\
3.829 \\
8.057 \\
11.522 \\
3.118 \\
7.747 \\
3.072\end{array}$ \\
\hline
\end{tabular}

HURRICANE, MERCED COUNTY, CAIIF.

About 12 miles by wagon road northeast of Merced. To reach it take road east from Merced to Tuttle, a station on the Atchison, Topeka \& Santa Fe Ry. about 5 miles from Merced, thence go north to Mr. $\Lambda$ pplegate's ranch house, continue north 0.25 mile to fence corner, and thence northeast 2 miles to signal. Signal is on a very prominent hill that can be seen from Mr. Applegate's house. The old signal erected by C. L. Nelson was blown down, but the new signal is close to where the old one stood.

Signal: Tripod with cross targets.

Station mark: Iron bench-mark post centered under signal.

[Latitude $37^{\circ} 21^{\prime} 19.214^{\prime \prime}$. Longitude $120^{\circ} 19^{\prime} 58.253^{\prime \prime}$.]

\begin{tabular}{|c|c|c|c|c|}
\hline To station- & Azimuth. & Back azimuth. & Distar & ce. \\
\hline 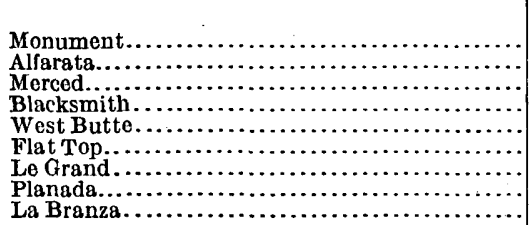 & \begin{tabular}{rcc}
\multicolumn{1}{c}{} & $\prime$ & $\prime \prime$ \\
31 & 06 & 50.86 \\
47 & 03 & 04.64 \\
68 & 04 & 59.67 \\
141 & 25 & 12.29 \\
230 & 36 & 39.87 \\
295 & 07 & 08.26 \\
319 & 50 & 36.40 \\
353 & 30 & 53.67 \\
357 & 04 & 39.95
\end{tabular} & \begin{tabular}{rcc}
\multicolumn{1}{c}{} & \multicolumn{1}{c}{ ' } \\
211 & 05 & 12.59 \\
226 & 59 & 15.20 \\
247 & 59 & 31.11 \\
321 & 23 & 18.44 \\
50 & 40 & 18.46 \\
115 & 15 & 13.87 \\
139 & 53 & 55.14 \\
173 & 31 & 14.12 \\
177 & 05 & 04.87
\end{tabular} & \begin{tabular}{r|} 
Log. meters. \\
3.8879045 \\
4.1051387 \\
4.1578254 \\
3.8689719 \\
4.0589318 \\
4.3384349 \\
4.0978411 \\
3.8666605 \\
4.2990338
\end{tabular} & $\begin{array}{r}\text { Miles. } \\
4.800 \\
7.916 \\
8.937 \\
4.595 \\
7.117 \\
13.545 \\
7.784 \\
4.571 \\
12.370\end{array}$ \\
\hline
\end{tabular}




\section{INDIAN, FRESNO COUNTY, CALIF.}

On a bald mountain at the head of Indian Valley and on the north side of Panoche Creek. It is about 7 miles northeast by east of Panoche post office. The PanocheMendota road intersects the road from Idria 4 miles south of the Kern oil well, from which the signal can be seen, probably 5 miles distant. Animals can be ridden up almost any of the ridges leading to highest point.

Signal: Cairn 7 feet high.

Station mark: Cairn 7 feet high.

Reference mark: Triangulation tablet set in rock; distance 1.60 meters from signal, azimuth $41^{\circ}$ to signal.

[Latitude $36^{\circ} 38^{\prime} 27.298^{\prime \prime}$. Longitude $120^{\circ} 43^{\prime} 34.341^{\prime \prime}$.]

\begin{tabular}{|c|c|c|c|c|}
\hline To station- & Azimuth. & Back azimuth. & Dista & ince. \\
\hline 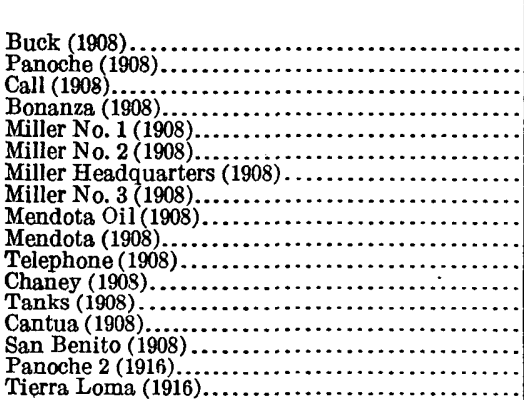 & $\begin{array}{rcc}\circ & \prime & \prime \prime \\
50 & 58 & 13.48 \\
64 & 03 & 24.40 \\
83 & 34 & 52.92 \\
128 & 57 & 25.73 \\
175 & 34 & 45.82 \\
197 & 47 & 18.15 \\
218 & 44 & 00.83 \\
229 & 02 & 20.90 \\
242 & 45 & 45.88 \\
248 & 41 & 18.18 \\
253 & 17 & 06.26 \\
263 & 42 & 39.39 \\
271 & 28 & 51.98 \\
294 & 18 & 30.89 \\
346 & 12 & 58.83 \\
160 & 21 & 21.01 \\
212 & 23 & 54.00\end{array}$ & \begin{tabular}{rcc}
\multicolumn{1}{c}{} & $\prime$ & $\prime \prime$ \\
230 & 52 & 59.42 \\
243 & 59 & 32.66 \\
263 & 22 & 48.63 \\
308 & 48 & 18.34 \\
355 & 33 & 56.50 \\
17 & 50 & 11.11 \\
38 & 50 & 06.50 \\
49 & 08 & 18.84 \\
62 & 57 & 32.37 \\
68 & 54 & 00.24 \\
73 & 24 & 41.49 \\
83 & 48 & 00.67 \\
91 & 43 & 41.41 \\
114 & 31 & 04.13 \\
166 & 15 & 54.86 \\
340 & 20 & 00.00 \\
32 & 26 & 21.73
\end{tabular} & \begin{tabular}{|c} 
Log. meters. \\
4.2270767 \\
4.0309438 \\
4.4823341 \\
4.4651214 \\
4.4229378 \\
4.3701193 \\
4.3842510 \\
4.2938859 \\
4.5180460 \\
4.5309150 \\
4.2957845 \\
4.1287127 \\
4.5687108 \\
4.5379847 \\
4.4907897 \\
4.0004416 \\
4.0588652
\end{tabular} & 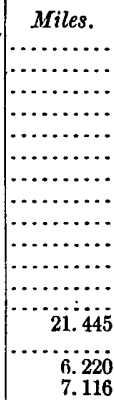 \\
\hline
\end{tabular}

INGOMAR, MERCED COUNTY, CALIF.

A red water tank about 50 feet high in yard of section house at Ingomar, a station on the Southern Pacific R. R. on west side of San Joaquin Valley.

Signal and station mark: Center of tank.

[Latitude $37^{\circ} 10^{\prime} 47.702^{\prime \prime}$. Longitude $120^{\circ} 58^{\prime} 04.631^{\prime \prime}$.]

\begin{tabular}{|c|c|c|c|c|}
\hline Tostation- & Azimuth. & Back azimuth. & Dista & ce. \\
\hline 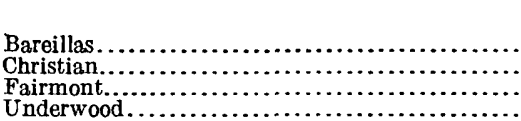 & \begin{tabular}{rcc}
\multicolumn{1}{c}{} & $\prime$ & $\prime \prime$ \\
20 & 14 & 31.54 \\
97 & 39 & 17.07 \\
234 & 40 & 33.03 \\
263 & 08 & 15.13
\end{tabular} & \begin{tabular}{rcc}
\multicolumn{1}{c}{0} &, & $\prime \prime$ \\
200 & 11 & 57.82 \\
277 & 34 & 42.96 \\
54 & 43 & 56.54 \\
83 & 12 & 56.29
\end{tabular} & \begin{tabular}{|r|} 
Log. meters. \\
4.2601790 \\
4.0525214 \\
4.0072640 \\
4.0627718
\end{tabular} & $\begin{array}{r}\text { Miles. } \\
11.312 \\
7.012 \\
6.318 \\
7.180\end{array}$ \\
\hline
\end{tabular}

ITALIAN SERVICE COLONY, MADERA COUNTY, CALIF. (Not occupied.)

Station mark: Center of large water tank at the Italian Service Colony, about 4 miles southwest of Madera.

[Latitude $36^{\circ} 55^{\prime} 55.31^{\prime \prime}$. Longitude $120^{\circ} 06^{\prime} 23.729^{\prime \prime}$.]

\begin{tabular}{|c|c|c|c|c|}
\hline Tostation- & Azimuth. & Back azimuth. & Dista & ce. \\
\hline 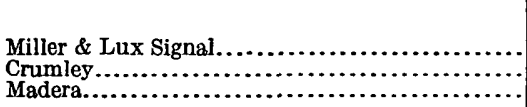 & \begin{tabular}{rcc}
\multicolumn{1}{c}{} & $\prime$ & $\prime \prime$ \\
92 & 27 & 58.10 \\
150 & 28 & 18.92 \\
232 & 34 & 34.25
\end{tabular} & \begin{tabular}{rcc}
\multicolumn{1}{c}{} & $\prime$ & $\prime \prime$ \\
272 & 25 & 41.38 \\
330 & 26 & 08.42 \\
52 & 36 & 13.01
\end{tabular} & \begin{tabular}{|r|} 
Log. meters. \\
3. 7509843 \\
4. 0367511 \\
3. 7091331
\end{tabular} & $\begin{array}{r}\text { Miles. } \\
3.502 \\
6.762 \\
3.180\end{array}$ \\
\hline
\end{tabular}

JAMESAN, FRESTO COUNTY, CALIF.

Station is 0.5 mile north of Jamesan station on the Southern Pacific R. R., and is about 8 miles west of Kerman. The signal is 200 feet south of the Kerman-Mendota road and 100 feet west of road to Jamesan. 
Signal: A tripod 18 feet high with cross targets.

Station mark: Iron bench-mark post centered under signal.

[Latitude $36^{\circ} 44^{\prime} 03.380^{\prime \prime}$. Longitude $120^{\circ} 12^{\prime} 17.039^{\prime \prime}$.]

\begin{tabular}{|c|c|c|c|c|}
\hline Tostation- & Azimuth. & Back azimuth. & Dista & \\
\hline 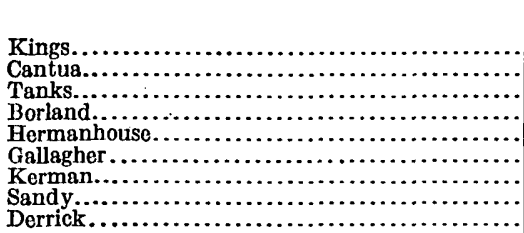 & \begin{tabular}{rcc}
\multicolumn{1}{c}{} & $\prime$ & $\prime \prime$ \\
19 & 34 & 15.20 \\
31 & 48 & 02.27 \\
40 & 09 & 07.99 \\
60 & 48 & 51.34 \\
97 & 21 & 10.56 \\
224 & 10 & 29.16 \\
275 & 15 & 06.22 \\
324 & 19 & 16.87 \\
336 & 23 & 07.66
\end{tabular} & \begin{tabular}{rcc}
\multicolumn{1}{c}{} & $\prime$ & $\prime \prime$ \\
199 & 33 & 27.31 \\
211 & 41 & 56.43 \\
220 & 05 & 19.05 \\
240 & 44 & 25.56 \\
277 & 17 & 11.43 \\
44 & 13 & 42.21 \\
95 & 20 & 17.00 \\
144 & 22 & 09.42 \\
156 & 25 & 30.78
\end{tabular} & $\begin{array}{r}\text { Log. meters. } \\
3.7736912 \\
4.4617664 \\
4.1732567 \\
4.1019662 \\
4.0000079 \\
4.0596341 \\
4.1122798 \\
4.0899247 \\
4.1722098 .\end{array}$ & $\begin{array}{r}\text { Miles. } \\
3.690 \\
17.994 \\
9.260 \\
7.858 \\
6.214 \\
7.128 \\
8.047 \\
7.643 \\
9.238\end{array}$ \\
\hline
\end{tabular}

JOKNSON, MADERA COUNTY, CALIF.

On a levee about 2 miles east of the bridge across San Joaquin River and 8 miles northeast of Dos Palos. It is about 0.4 mile south of an east and west road leading to Chowchilla.

Signal: Timber structure with cross targets and flag.

Station mark: Iron bench-mark post centered under signal.

[Latitude $37^{\circ} 03^{\prime} 51.386^{\prime \prime}$. Longitude $120^{\circ} 30^{\prime} 50.424^{\prime \prime}$.]

\begin{tabular}{|c|c|c|c|c|}
\hline Mostation- & Azimuth. & Back azimuth. & Distar & \\
\hline 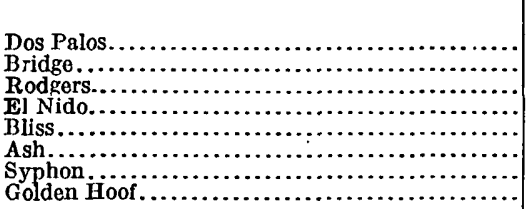 & \begin{tabular}{rcc}
\multicolumn{1}{c}{} & $\prime$ & $\prime \prime$ \\
49 & 13 & 16.17 \\
72 & 37 & 18.02 \\
174 & 06 & 17.46 \\
194 & 46 & 51.86 \\
236 & 59 & 30.05 \\
262 & 43 & 16.03 \\
300 & 59 & 52.97 \\
354 & 32 & 17.49
\end{tabular} & \begin{tabular}{rcc}
\multicolumn{1}{c}{} & $\prime$ & $\prime \prime$ \\
229 & 10 & 14.95 \\
252 & 36 & 03.07 \\
354 & 05 & 59.58 \\
14 & 47 & 42.07 \\
57 & 03 & 25.13 \\
82 & 48 & 09.25 \\
121 & 04 & 59.95 \\
174 & 33 & 45.71
\end{tabular} & \begin{tabular}{|r|} 
Log. meters. \\
4.1165906 \\
3.5078316 \\
3.8528015 \\
3.9059507 \\
4.0597503 \\
4.0832402 \\
4.1674508 \\
4.0871288
\end{tabular} & $\begin{array}{r}\text { Miles. } \\
8.127 \\
2.001 \\
4.427 \\
5.004 \\
7.130 \\
7.526 \\
9.137 \\
7.594\end{array}$ \\
\hline
\end{tabular}

JOLON, MONTEREY COUNTY, CALIF.

A three-point station on steep hill 1 mile east of Jolon, in San Antonio Valley. Leave San Antonio Mission road at a point opposite Earle Brothers' ranch, about 3 miles southeast of Mission and 4 miles northwest of Jolon.

Station mark: A mission roof tile set in ground under small rock cairn. Instrument over mark.

[Latitude $35^{\circ} 59^{\prime} 14.431^{\prime \prime}$. Longitude $121^{\circ} 11^{\prime} 55.417^{\prime \prime}$.]

\begin{tabular}{|c|c|c|c|c|}
\hline Tostation- & Azimuth. & Back azimuth. & Dista & \\
\hline 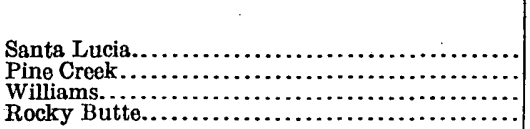 & $\begin{array}{ccc}\circ & \prime \prime & \prime \prime \\
131 & 42 & 58.53 \\
168 & 30 & 18.46 \\
282 & 23 & 51.69 \\
340 & 28 & 15.18\end{array}$ & $\begin{array}{ccc}\circ & \prime \prime & \prime \prime \\
311 & 35 & 13.13 \\
348 & 29 & 16.88 \\
102 & 30 & 49.89 \\
160 & 33 & 09.81\end{array}$ & $\begin{array}{r}\text { Log. meters. } \\
4.4228507 \\
4.1184733 \\
4.2617385 \\
4.5783184\end{array}$ & $\begin{array}{r}\text { Miles. } \\
16.451 \\
8.162 \\
11.352 \\
23.533\end{array}$ \\
\hline
\end{tabular}

JUHL, SAN LUIS OBISPO COUNTY, CALIF.

On a timbered peak at the source of Old Creek, about 7 or 8 miles from Cayucos. The York Hill road from Paso Robles to Cayucos crosses summit near the source of Old Creek. At this summit there is a gate on either side of road and a grove of Monterey cypress trees on west side. Enter gate on east side of road, go east to summit of first hill, thence south along ridge, but do not go through fence until summit of hill is reached, about 0.75 mile from the road. All timber was cut from summit. 
Signal: A tree or stump with branches on it left standing.

Station mark: A triangulation tablet set in rock, placed in the ground. Position of tablet was computed. Azimuth from tablet to signal, $49^{\circ} 04^{\prime}$; distance, 1.12 meters.

[Latitude $35^{\circ} 30^{\prime} 19.973^{\prime \prime}$. Longitude $120^{\circ} 50^{\prime} 13.438^{\prime \prime}$.]

\begin{tabular}{|c|c|c|c|c|}
\hline Tostation- & Azimuth. & Back azimuth. & Dista & ce. \\
\hline 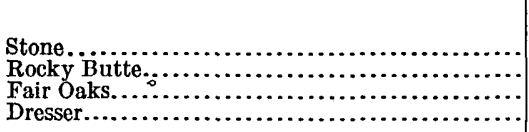 & \begin{tabular}{rcc}
\multicolumn{1}{c}{} & $\prime$ & \multicolumn{1}{c}{} \\
78 & 56 & 15.06 \\
131 & 30 & 28.44 \\
194 & 23 & 53.67 \\
250 & 25 & 12.97
\end{tabular} & \begin{tabular}{rcc}
\multicolumn{1}{c}{} & \multicolumn{1}{c}{$\quad \prime \prime$} \\
258 & 52 & 18.86 \\
311 & 22 & 43.70 \\
14 & 25 & 24.86 \\
70 & 34 & 26.80
\end{tabular} & $\begin{array}{r}\text { Log. meters. } \\
4.0190373 \\
4.4285017 \\
4.2002766 \\
4.4058368\end{array}$ & $\begin{array}{r}\text { Miles. } \\
6.492 \\
16.667 \\
9.854 \\
15.819\end{array}$ \\
\hline
\end{tabular}

KEARNEY, FRESNO COUNTY, CALIF.

A very high tower in Kearney Park about 6 miles west of Fresno. Signal and station mark: Center of tower.

[Latitude $36^{\circ} 43^{\prime} 20.442^{\prime \prime}$. Longitude $119^{\circ} 55^{\prime} 05.626^{\prime \prime}$.]

\begin{tabular}{|c|c|c|c|c|}
\hline Tostation- & Azimuth. & Back azimuth. & Dista & \\
\hline 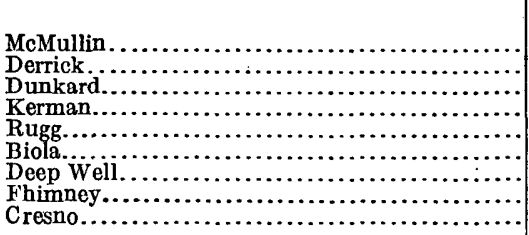 & \begin{tabular}{rcc}
\multicolumn{1}{c}{} & \multicolumn{1}{c}{ ' } \\
33 & 18 & 59.53 \\
58 & 02 & 14.14 \\
82 & 19 & 41.12 \\
90 & 37 & 19.87 \\
97 & 36 & 51.46 \\
135 & 12 & 10.77 \\
195 & 07 & 07.43 \\
225 & 35 & 02.97 \\
262 & 00 & 21.89
\end{tabular} & \begin{tabular}{rcc}
\multicolumn{1}{c}{} & $\prime$ & $\prime \prime$ \\
213 & 17 & 06.59 \\
237 & 54 & 21.32 \\
262 & 16 & 53.12 \\
270 & 32 & 13.88 \\
277 & 33 & 46.27 \\
315 & 08 & 42.51 \\
15 & 09 & 21.55 \\
45 & 38 & 40.73 \\
82 & 04 & 54.17
\end{tabular} & \begin{tabular}{|r} 
Log. meters. \\
$\mathbf{3 . 9 3 1 8 1 1 1}$ \\
4.3653550 \\
$\mathbf{3 . 8 4 7 3 6 9 5}$ \\
4.1037913 \\
$\mathbf{3 . 8 8 9 4 3 4 3}$ \\
4.0878869 \\
4.3523742 \\
4.1169640 \\
4.0697740
\end{tabular} & $\begin{array}{r}\text { Miles. } \\
5.311 \\
14.411 \\
4.372 \\
7.891 \\
4.817 \\
7.607 \\
13.987 \\
8.134 \\
7.297\end{array}$ \\
\hline
\end{tabular}

KERMAN, FRESNO COUNTY, CALIF.

Station is center of water tank in town of Kerman on Southern Pacific R. R., on west side of San Joaquin Valley.

[Latitude $36^{\circ} 43^{\prime} 24.610^{\prime}$. Longitude, $120^{\circ} 03^{\prime} 37.364^{\prime \prime}$.]

\begin{tabular}{|c|c|c|c|c|}
\hline To station- & Azimuth. & Back azimuth. & Dista & ce. \\
\hline 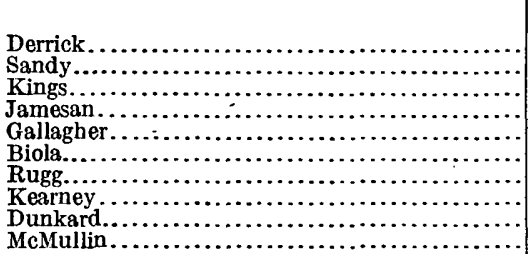 & $\begin{array}{rcc}\circ & \prime & \prime \prime \\
29 & 15 & 07.64 \\
33 & 05 & 57.42 \\
73 & 34 & 57.52 \\
95 & 20 & 17.00 \\
152 & 34 & 43.74 \\
205 & 23 & 05.25 \\
259 & 51 & 07.07 \\
270 & 32 & 13.88 \\
280 & 34 & 18.25 \\
312 & 12 & 08.25\end{array}$ & \begin{tabular}{rcc}
\multicolumn{1}{c}{} & \multicolumn{1}{c}{$\prime \prime$} \\
209 & 12 & 20.41 \\
213 & 03 & 39.50 \\
253 & 28 & 59.05 \\
275 & 15 & 06.22 \\
332 & 32 & 45.72 \\
25 & 24 & 43.26 \\
79 & 53 & 07.90 \\
90 & 37 & 19.87 \\
100 & 36 & 36.21 \\
132.15 & 21.07
\end{tabular} & $\begin{array}{r}\text { Log. meters. } \\
\text { 4.1535698 } \\
4.0212703 \\
4.1910270 \\
4.1122798 \\
4.0258373 \\
\mathbf{3 . 9 7 6 3 5 8 6} \\
3.7069745 \\
\mathbf{4 . 1 0 3 7 9 1 3} \\
\mathbf{3 . 7 6 5 3 4 5 2} \\
\mathbf{4 . 0 3 4 2 2 3 4}\end{array}$ & $\begin{array}{r}\text { Miles. } \\
\mathbf{8 . 8 5 0} \\
6.526 \\
\mathbf{9 . 6 4 7} \\
8.047 \\
6.595 \\
\mathbf{5 . 8 8 4} \\
\mathbf{3 . 1 6 5} \\
\mathbf{7 . 8 9 1} \\
\mathbf{3 . 6 2 0} \\
\mathbf{6 . 7 2 3}\end{array}$ \\
\hline
\end{tabular}

EINGS, FRESNO COUNTY, CALIF.

On west end of south pier of a wooden bridge across Kings Slough, about 3 miles southwest of Jamesan.

Signal and station mark: A 2 by 6 inch timber with cross targets and flag.

Reference mark: Iron bench-mark post on the levee at southwest corner of bridge. Position of signal computed: Azimuth from bench mark to signal, $258^{\circ} 27^{\prime}$; distance, 19.8 feet. 
[Latitude $36^{\circ} 41^{\prime} 01.843^{\prime \prime}$. Longitude $120^{\circ} 13^{\prime} 37.160^{\prime \prime}$.]

\begin{tabular}{|c|c|c|c|c|}
\hline Tostation- & Azimuth. & Back azimuth. & Dista & co. \\
\hline 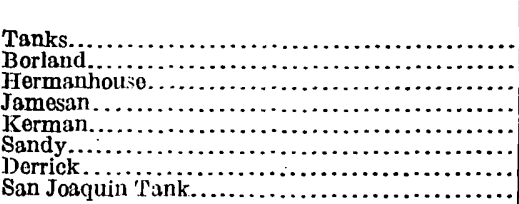 & \begin{tabular}{rcc}
\multicolumn{1}{c}{} & \multicolumn{1}{c}{} & $\prime \prime$ \\
52 & 43 & 59.33 \\
86 & 22 & 30.47 \\
130 & 55 & 02.44 \\
199 & 33 & 27.31 \\
253 & 28 & 59.05 \\
295 & 36 & 57.51 \\
315 & 16 & 44.34 \\
337 & 58 & 45.06
\end{tabular} & \begin{tabular}{rcc}
\multicolumn{1}{c}{} & $\prime$ & $\prime \prime$ \\
232 & 40 & 58.36 \\
266 & 18 & 52.71 \\
310 & 51 & 51.34 \\
19 & 34 & 15.20 \\
73 & 34 & 57.52 \\
115 & 40 & 37.79 \\
135 & 19 & 55.19 \\
158 & 00 & 08.29
\end{tabular} & $\begin{array}{r}\text { Log. meters. } \\
3.9810611 \\
3.9575854 \\
4.0209628 \\
3.7736912 \\
4.1910270 \\
4.0070398 \\
4.0528116 \\
3.9658451\end{array}$ & $\begin{array}{r}\text { Miles. } \\
5.949 \\
5.636 \\
6.521 \\
3.690 \\
9.647 \\
6.315 \\
7.017 \\
5.744\end{array}$ \\
\hline
\end{tabular}

KINGSBURY, FRESNO COUNTY, CALIF. (Not occupied.)

Station mark: High black tank in the town of Kingsbury.

[Latitude $36^{\circ} 30^{\prime} 53.750^{\prime \prime}$. Longitude $119^{\circ} 33^{\prime} 03.378^{\prime \prime}$.]

\begin{tabular}{|c|c|c|c|c|}
\hline Tostation- & Azimuth. & Back azimuth. & Dista & ce. \\
\hline 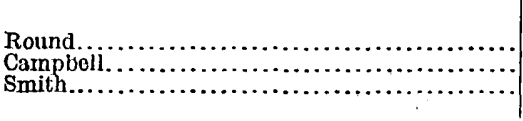 & $\begin{array}{ccc}\circ & \prime & \prime \prime \\
186 & 14 & 39.08 \\
210 & 01 & 47.80 \\
247 & 50 & 54.45\end{array}$ & $\begin{array}{rcc}\circ & \prime & \prime \prime \\
6 & 16 & 11.74 \\
30 & 06 & 26.65 \\
67 & 58 & 15.35\end{array}$ & \begin{tabular}{r|} 
Log. meters. \\
4.5309734 \\
4.3654139 \\
4.2982711
\end{tabular} & $\begin{array}{r}\text { Miles. } \\
21.102 \\
14.413 \\
12.349\end{array}$ \\
\hline
\end{tabular}

\section{IA BRANZA, MERCED COUNTY, CALIF.}

A tank at La Branza, a station on the Southern Pacific R. R. 12 miles southeast of Merced. There are two large silos about 300 feet southeast of tank.

Station mark and signal: Center of tank with a green roof and a green railing around it.

[Latitude $37^{\circ} 10^{\prime} 34.262^{\prime \prime}$. Longitude $120^{\circ} 19^{\prime} 17.111^{\prime \prime}$.]

\begin{tabular}{|c|c|c|c|c|}
\hline & Azimuth. & Back azimuth. & Dista & \\
\hline 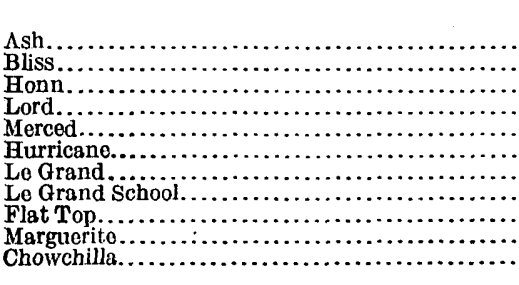 & \begin{tabular}{rcc}
\multicolumn{1}{c}{} & $\prime$ & $\prime \prime$ \\
25 & 06 & 12.32 \\
50 & 28 & 59.19 \\
71 & 58 & 40.69 \\
95 & 39 & 48.31 \\
135 & 19 & 02.02 \\
177 & 05 & 04.87 \\
214 & 25 & 34.31 \\
226 & 16 & 09.34 \\
240 & 25 & 24.07 \\
277 & 05 & 38.60 \\
315 & 51 & 51.96
\end{tabular} & \begin{tabular}{rcc}
\multicolumn{1}{c}{$\bullet$} & $\prime$ & $\prime \prime$ \\
205 & 04 & 07.55 \\
230 & 25 & 56.00 \\
251 & 53 & 50.54 \\
275 & 35 & 17.39 \\
315 & 13 & 09.23 \\
357 & 04 & 39.95 \\
34 & 28 & 27.75 \\
46 & 18 & 40.42 \\
60 & 33 & 03.79 \\
97 & 10 & 59.06 \\
135 & 54 & 03.62
\end{tabular} & $\begin{array}{r}\text { Log. meters. } \\
4.0804456 \\
3.9871177 \\
4.0957696 \\
4.0457740 \\
4.3099650 \\
4.2990338 \\
4.0967741 \\
3.9306819 \\
4.3330243 \\
4.1201998 \\
3.8880722\end{array}$ & $\begin{array}{r}\text { Miles. } \\
7.478 \\
6.032 \\
7.747 \\
6.904 \\
12.686 \\
12.370 \\
7.765 \\
5.297 \\
1.3 .377 \\
8.195 \\
4.802\end{array}$ \\
\hline
\end{tabular}

\section{LAS PALMAS, FRESAO COUNTY, CALIF.}

A tank on top of large tank house in Las Palmas, a town on Friant Branch of Southern Pacific R. R., 7 miles east of Fresno. The tank house stands back of Las Palmas Winery.

Signal: Center of tank.

Station mark: Center of concrete foundation for building.

[Latitude $36^{\circ} 45^{\prime} 25.888^{\prime \prime}$. Longitude $119^{\circ} 41^{\prime} 53.001^{\prime \prime}$.]

\begin{tabular}{|c|c|c|c|c|}
\hline Tostation- & Azimuth. & Back azimuth. & Dista & \\
\hline 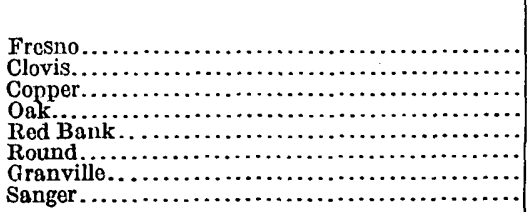 & $\begin{array}{ccc}\circ & , & 1 P \\
74 & 26 & 52.16 \\
179 & 30 & 38.21 \\
193 & 30 & 48.63 \\
213 & 11 & 14.28 \\
230 & 10 & 34.34 \\
247 & 43 & 11.22 \\
283 & 01 & 37.02 \\
292 & 56 & 21.46\end{array}$ & 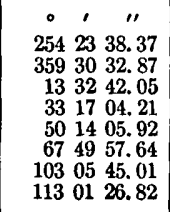 & \begin{tabular}{|c} 
Log. meters. \\
3.9212635 \\
3.8698088 \\
4.3017111 \\
4.4210865 \\
4.0569118 \\
4.2595383 \\
4.0235123 \\
4.1386317
\end{tabular} & $\begin{array}{r}\text { Miles. } \\
5.183 \\
4.604 \\
12.447 \\
16.384 \\
7.084 \\
11.295 \\
6.559 \\
8.550\end{array}$ \\
\hline
\end{tabular}


IAST CHATCE, MADERA COUNTY, CALIF.

A windmill on the Last Chance ranch, about 2 miles southwest of Levine School and 10 miles southwest of Madera.

Signal: Windmill.

Station mark: Center of 8-inch well under mill.

[Latitude $36^{\circ} 51^{\prime} 42.852^{\prime \prime}$. Longitude $120^{\circ} 09^{\prime} 17.009^{\prime \prime}$.]

\begin{tabular}{|c|c|c|c|c|}
\hline Tostation- & Azimuth. & Back azimuth. & Dista & ce. \\
\hline 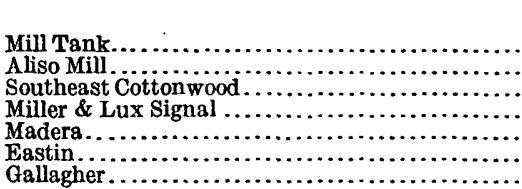 & \begin{tabular}{rcc}
\multicolumn{1}{c}{ ' } & \multicolumn{1}{c}{ " } \\
22 & 41 & 31.73 \\
89 & 52 & 13.64 \\
134 & 31 & 19.05 \\
170 & 28 & 29.22 \\
217 & 26 & 10.08 \\
267 & 45 & 57.19 \\
329 & 15 & 38.28
\end{tabular} & \begin{tabular}{rll}
$\circ$ & \multicolumn{1}{c}{,} & \multicolumn{1}{c}{ " } \\
202 & 40 & 22.54 \\
269 & 48 & 35.19 \\
314 & 27 & 47.962 \\
350 & 27 & 56.53 \\
37 & 29 & 32.66 \\
87 & 51 & 11.42 \\
149 & 17 & 03.68
\end{tabular} & $\begin{array}{r}\text { Log. meters. } \\
3.8702547 \\
3.9552171 \\
4.0864039 \\
3.9107280 \\
4.1376429 \\
4.1134044 \\
3.8394367\end{array}$ & $\begin{array}{r}\text { Miles. } \\
4.609 \\
5.605 \\
7.581 \\
5.059 \\
8.531 \\
8.068 \\
4.293\end{array}$ \\
\hline
\end{tabular}

IE GRAND, MERCED COUNTY, CALIF.

On a hill 200 feet east of a north and south road and 3 miles north of Le Grand, a station on the Atchison, Topeka \& Santa Fe Ry. The property is owned by W. C. Dallas.

Signal: A quadripod.

Station mark: Iron bench-mark post centered under signal.

[Latitude $37^{\circ} 16^{\prime} 08.524^{\prime \prime}$. Longitude $120^{\circ} 14^{\prime} 30.383^{\prime \prime}$.]

\begin{tabular}{|c|c|c|c|c|}
\hline To station- & Arimuth. & Back azimuth. & Dista & \\
\hline 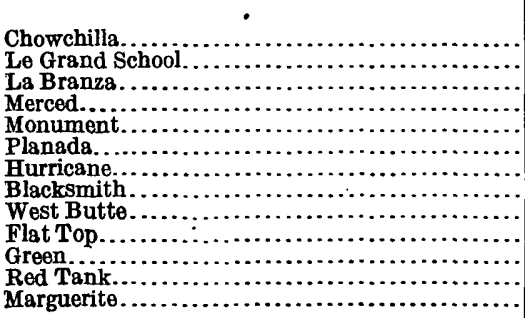 & $\begin{array}{rcc}\circ & \prime & \prime \prime \\
6 & 06 & 28.96 \\
11 & 37 & 44.24 \\
34 & 28 & 27.75 \\
101 & 09 & 47.63 \\
103 & 50 & 07.34 \\
107 & 24 & 33.27 \\
139 & 53 & 55.14 \\
140 & 29 & 02.11 \\
182 & 41 & 05.26 \\
268 & 28 & 51.22 \\
270 & 52 & 11.55 \\
308 & 29 & 24.97 \\
333 & 16 & 20.65\end{array}$ & \begin{tabular}{rcc}
\multicolumn{1}{c}{} & $\prime$ & $\prime \prime$ \\
186 & 05 & 47.42 \\
191 & 37 & 21.94 \\
214 & 25 & 34.31 \\
281 & 01 & 00.76 \\
283 & 45 & 10.56 \\
287 & 21 & 35.11 \\
319 & 50 & 36.40 \\
320 & 23 & 49.51 \\
2 & 41 & 24.74 \\
88 & 33 & 37.80 \\
91 & 01 & 19.60 \\
128 & 32 & 59.79 \\
153 & 18 & 48.04
\end{tabular} & $\begin{array}{r}\text { Log. meters. } \\
4.2025688 \\
3.6538189 \\
4.0967741 \\
4.3390540 \\
4.0943978 \\
3.8804168 \\
4.0978411 \\
4.2993034 \\
4.2268546 \\
4.0668279 \\
4.3483662 \\
4.0486003 \\
4.1261244\end{array}$ & $\begin{array}{r}\text { Miles. } \\
9.906 \\
2.800 \\
7.765 \\
13.565 \\
7.722 \\
4.718 \\
7.784 \\
12.378 \\
10.476 \\
7.247 \\
13.858 \\
6.950 \\
8.308\end{array}$ \\
\hline
\end{tabular}

LE GRAND SCHOOL, MERCED COUNTY, CALIF.

Station mark and signal: Cupola on schoolhouse in the town of Le Grand on the Atchison, Topeka \& Santa Fe Ry.

[Latitude $37^{\circ} 13^{\prime} 45.346^{\prime \prime}$. Longitude $120^{\circ} 15^{\prime} 07.230^{\prime \prime}$.]

\begin{tabular}{|c|c|c|c|c|}
\hline To station- & Azimuth. & Back azimuth. & Dista & \\
\hline 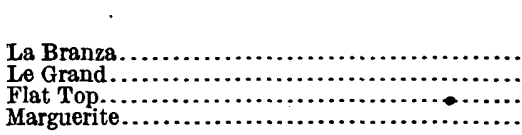 & $\begin{array}{rcc}\circ & \prime & \prime \prime \\
46 & 18 & 40.42 \\
191 & 37 & 21.94 \\
249 & 23 & 50.78 \\
317 & 23 & 45.09\end{array}$ & \begin{tabular}{rcc}
\multicolumn{1}{c}{} & $\prime$ & $\prime \prime$ \\
226 & 16 & 09.34 \\
11 & 37 & 44.24 \\
69 & 28 & 59.53 \\
137 & 26 & 34.69
\end{tabular} & \begin{tabular}{|r|} 
Log. meters. \\
3.9306819 \\
3.6538189 \\
4.1279427 \\
4.0097013
\end{tabular} & $\begin{array}{r}\text { Miles. } \\
5.297 \\
2.800 \\
8.342 \\
6.354\end{array}$ \\
\hline
\end{tabular}


LONE GUM, FRESNO COUNTY, CALIF. (Not occupied.)

Station mark: Center of large eucalyptus tree on flume line northeast of Clovis.

[Latitude $36^{\circ} 51^{\prime} 25.736^{\prime \prime}$. Longitude $119^{\circ} 38^{\prime} 15.289^{\prime \prime}$.]

\begin{tabular}{|c|c|c|c|c|}
\hline To station- & Azimuth. & Back azimuth. & Distar & ce. \\
\hline 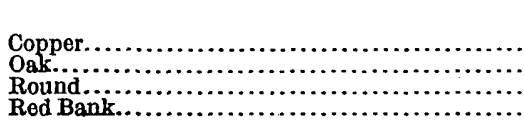 & $\begin{array}{ccc}\circ & \prime & \prime \prime \\
175 & 10 & 43.91 \\
219 & 31 & 07.71 \\
290 & 13 & 37.66 \\
318 & 28 & 59.91\end{array}$ & $\begin{array}{rcc}\circ & \prime & \prime \prime \\
355 & 10 & 26.76 \\
39 & 34 & 47.31 \\
110 & 18 & 14.03 \\
138 & 30 & 21.20\end{array}$ & $\mid$\begin{tabular}{r|} 
Log. meters. \\
3. 9249409 \\
4. 1528344 \\
4. 0854889 \\
3.7049268
\end{tabular} & $\begin{array}{r}\text { Miles. } \\
\mathbf{5 . 2 2 7} \\
8.835 \\
7.566 \\
\mathbf{3 . 1 5 0}\end{array}$ \\
\hline
\end{tabular}

LONE PINE, SAN BENITO COUNTY, CALIF. (Not occupled.)

A prominent pine tree on brushy hill almost on line between Harris and Martin and about midway between them.

Station mark: Pine tree.

[Latitude $36^{\circ} 31^{\prime} 17.024^{\prime \prime}$. Longitude $121^{\circ} 10^{\prime} 06.010^{\prime \prime}$.]

\begin{tabular}{|c|c|c|c|c|}
\hline Tostation- & Azimuth. & Back azimuth. & Dista & ce. \\
\hline 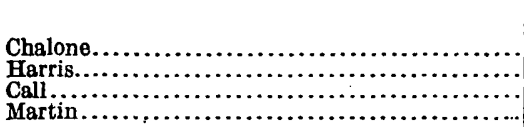 & $\begin{array}{rcc}\circ & \prime & \prime \prime \\
16 & 20 & 35.64 \\
126 & 12 & 14.29 \\
223 & 41 & 49.00 \\
302 & 23 & 17.73\end{array}$ & \begin{tabular}{rcc}
\multicolumn{1}{c}{} & \multicolumn{1}{c}{} & $\prime \prime$ \\
196 & 19 & 38.67 \\
306 & 10 & 21.70 \\
43 & 45 & 33.95 \\
122 & 25 & 44.54
\end{tabular} & $\begin{array}{r}\text { Log. meters. } \\
\text { 3. } 9283823 \\
\text { 3.7656121 } \\
\text { 4. } 1330026 \\
\text { 3. } 8618133\end{array}$ & $\begin{array}{r}\text { Miles. } \\
5.269 \\
3.622 \\
8.440 \\
4.520\end{array}$ \\
\hline
\end{tabular}

LORD, MERCED COUNTY, CAIIF.

To reach station follow road due south from Merced for 8 miles to a $T$ road thence east for 2 miles. The signal is on the fence line along county road and is 0.5 mile south of a small cabin and barn in Mr. Lord's pasture field.

Signal: Tripod with cross targets.

Station mark: Iron bench-mark post centered under signal.

[Latitude $37^{\circ} 11^{\prime} 09.596^{\prime \prime}$. Longitude $120^{\circ} 26^{\prime} 45.397^{\prime \prime}$.]

\begin{tabular}{|c|c|c|c|c|}
\hline To station- & Azimuth. & Back azimuth. & Distar & ce. \\
\hline 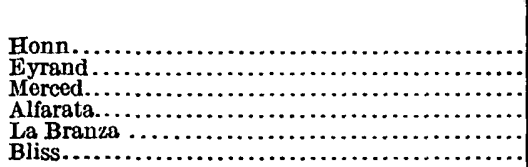 & \begin{tabular}{rcc}
$\circ$ & \multicolumn{1}{c}{} & \multicolumn{1}{c}{} \\
9 & 04 & 44.11 \\
115 & 52 & 11.55 \\
166 & 06 & 51.56 \\
183 & 59 & 40.10 \\
275 & 35 & 17.39 \\
333 & 37 & 32.95
\end{tabular} & $\begin{array}{rcc}\circ & \prime & \prime \prime \\
189 & 04 & 24.74 \\
295 & 48 & 17.73 \\
346 & 05 & 30.06 \\
3 & 59 & 47.4^{4} \\
95 & 39 & 48.31 \\
153 & 49 & 00.49\end{array}$ & $\begin{array}{r}\text { Log. meters. } \\
3.7004943 \\
4.0250777 \\
4.1404688 \\
4.0057353 \\
4.0457740 \\
3.9085808\end{array}$ & $\begin{array}{r}\text { Miles. } \\
3.118 \\
6.583 \\
8.587 \\
6.296 \\
6.904 \\
5.034\end{array}$ \\
\hline
\end{tabular}

LOS BANOS TANK, MERCED COUNTY, CAIIF.

The city water tank on the southeast side of the town of Los Banos.

Signal: Center of tank.

Station mark: Center of tank.

[Latitude $37^{\circ} 03^{\prime} 39.278^{\prime \prime}$. Longitude $120^{\circ} 50^{\prime} 42.805^{\prime \prime}$.]

\begin{tabular}{|c|c|c|c|c|}
\hline To station- & Azimuth. & Back azimuth. & Distar & \\
\hline 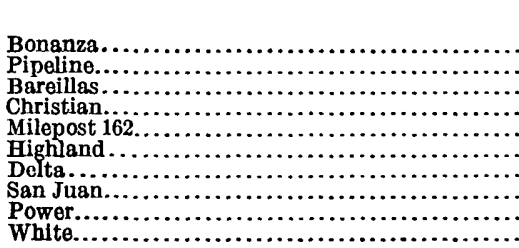 & $\begin{array}{rcc}\circ & \prime & \prime \prime \\
23 & 06 & 54.01 \\
67 & 47 & 08.65 \\
77 & 22 & 17.66 \\
123 & 42 & 32.58 \\
170 & 20 & 18.55 \\
211 & 36 & 41.22 \\
236 & 18 & 16.64 \\
262 & 27 & 08.23 \\
273 & 07 & 19.41 \\
358 & 39 & 22.92\end{array}$ & $\begin{array}{rcc}\circ & \prime & \prime \prime \\
203 & 02 & 01.39 \\
247 & 43 & 30.34 \\
257 & 15 & 17.99 \\
303 & 33 & 32.17 \\
350 & 19 & 45.97 \\
31 & 39 & 32.93 \\
56 & 22 & 06.81 \\
82 & 33 & 57.46 \\
93 & 11 & 53.77 \\
178 & 39 & 32.25\end{array}$ & \begin{tabular}{|r|} 
Log. meters. \\
4.4878415 \\
3.9856751 \\
4.2466538 \\
4.4239815 \\
3.9000410 \\
4.1269623 \\
4.0540401 \\
4.2282697 \\
4.0517582 \\
4.2140071
\end{tabular} & $\begin{array}{r}\text { Miles. } \\
19.107 \\
6.012 \\
10.965 \\
16.494 \\
4.936 \\
8.324 \\
7.037 \\
10.510 \\
7.000 \\
10.171\end{array}$ \\
\hline
\end{tabular}


IUCERNE, MERCED COUNTY, CALIF, (Not occupied.)

Station mark: Center of windmill at Miller \& Lux Lucerne ranch, north of Dos Palos.

[Latitude $37^{\circ} 05^{\prime} 14.366^{\prime \prime}$. Longitude $120^{\circ} 35^{\prime} 37.239^{\prime \prime}$.]

\begin{tabular}{|c|c|c|c|c|}
\hline Tostation- & Azimuth. & Back azimuth. & Distar & \\
\hline 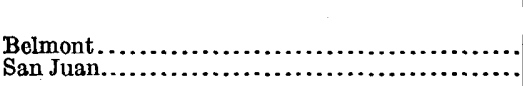 & $\begin{array}{lcc}\circ & \prime & \prime \prime \\
25 & 21 & 26.25 \\
82 & 37 & 44.66\end{array}$ & $\begin{array}{ccc}\circ & \prime & \prime \prime \\
205 & 20 & 22.17 \\
262 & 35 & 27.97\end{array}$ & \begin{tabular}{|c|} 
Log. meters. \\
3.7878512 \\
3.7517153
\end{tabular} & $\begin{array}{r}\text { Miles. } \\
3.812 \\
3.508\end{array}$ \\
\hline
\end{tabular}

IYNCE, SAN LUIS OBISPO COUNTY, CALIF.

On a brushy mountain, locally known as Lynch Mountain or Tierra Redondo. It is about 7 miles south of Pleyto post office, and there is a good road from that place to the Lynch ranch, at foot of mountain. Animals might be taken to summit, but it is probably better to walk up on the west side, keeping near the summit, but not on top until very near the signal. The top of the mountain is rather flat and covered with thick brush, but signal is on the highest point and near the cliff on the southwest end.

Signal: An oak tree set in the ground.

Station mark: A triangulation tablet set in solid rock 1.05 meters a little east of north of signal. Position of tablet was computed. This point is not the same as point called Lynch by L. F. Biggs. Azimuth from tablet to signal, $24^{\circ} 00^{\prime}$; distance, 1.05 meters.

[Latitude $35^{\circ} 46^{\prime} 16.765^{\prime \prime}$. Longitude $120^{\circ} 59^{\prime} 06.972^{\prime \prime}$.]

\begin{tabular}{|c|c|c|c|c|}
\hline To station- & Azimuth. & Back azimuth. & Distar & \\
\hline 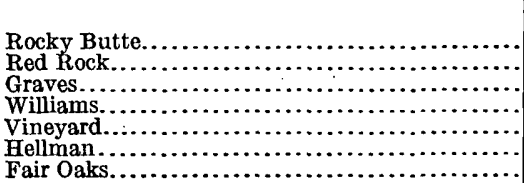 & \begin{tabular}{rcc}
\multicolumn{1}{c}{} & $\prime \prime$ & $\prime \prime$ \\
29 & 36 & 37.88 \\
84 & 09 & 17.14 \\
129 & 29 & 23.34 \\
175 & 57 & 38.95 \\
266 & 44 & 07.46 \\
267 & 44 & 39.13 \\
309 & 05 & 42.01
\end{tabular} & \begin{tabular}{rcc}
\multicolumn{1}{c}{} & $\prime \prime$ \\
209 & 34 & 03.13 \\
264 & 03 & 10.23 \\
309 & 24 & 31.63 \\
355 & 57 & 05.89 \\
86 & 55 & 03.93 \\
87 & 52 & 15.54 \\
129 & 12 & 24.90
\end{tabular} & $\begin{array}{r}\text { Log. meters. } \\
\text { 4. } 1301415 \\
4.2001510 \\
4.2096584 \\
4.3027095 \\
4.4509332 \\
4.2927839 \\
4.3497869\end{array}$ & $\begin{array}{r}\text { Miles. } \\
8.385 \\
9.851 \\
10.069 \\
12.476 \\
17.550 \\
12.194 \\
13.904\end{array}$ \\
\hline
\end{tabular}

M'MULLIN, FRESNO COUNTY, CALIF.

A white tank house at McMullin station 7 miles southeast of Kerman.

Signal: Center of tank.

Station mark: Center of concrete foundation.

[Latitude $36^{\circ} 39^{\prime} 28.696^{\prime \prime}$. Longitude $119^{\circ} 58^{\prime} 14.644^{\prime \prime}$.]

\begin{tabular}{|c|c|c|c|c|}
\hline To station- & Azimuth. & Back azimuth. & Distan & ce. \\
\hline 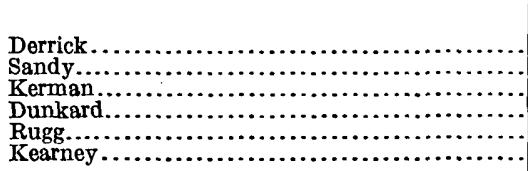 & \begin{tabular}{rcc}
\multicolumn{1}{c}{} & $\prime$ & $\prime \prime$ \\
71 & 02 & 55.60 \\
83 & 42 & 22.96 \\
132 & 15 & 21.07 \\
159 & 47 & 28.68 \\
159 & 52 & 09.67 \\
213 & 17 & 06.59
\end{tabular} & \begin{tabular}{rcc}
\multicolumn{1}{c}{$\circ$} & $\prime$ & $\prime \prime$ \\
250 & 56 & 55.93 \\
263 & 36 & 52.50 \\
312 & 12 & 08.25 \\
339 & 46 & 33.74 \\
339 & 50 & 57.57 \\
33 & 18 & 59.53
\end{tabular} & \begin{tabular}{r|} 
Loy. meters. \\
4. 1996952 \\
4.1409783 \\
4.0342234 \\
3.8201001 \\
3.9394826 \\
3.9318111
\end{tabular} & $\begin{array}{r}\text { Miles. } \\
9.841 \\
8.597 \\
6.723 \\
4.106 \\
5.405 \\
5.311\end{array}$ \\
\hline
\end{tabular}

MADERA, MADERA COUNTY, CALIF., FROM SOUTH.

Madera County courthouse at Madera, Calif.

Signal and station mark: Center of clock tower and dome. Center of cupola computed. 
[Latitude $36^{\circ} 57^{\prime} 36.362^{\prime \prime}$ (from south), $36^{\circ} 57^{\prime} 36.402^{\prime \prime}$ (from north). Longitude $120^{\circ} 03^{\prime} 39.663^{\prime \prime}$ (from south), $120^{\circ} 03^{\prime} 39.421^{\prime \prime}$ (from north).]

\begin{tabular}{|c|c|c|c|c|}
\hline Tostation- & Azimuth. & Back azimuth. & Dista & \\
\hline 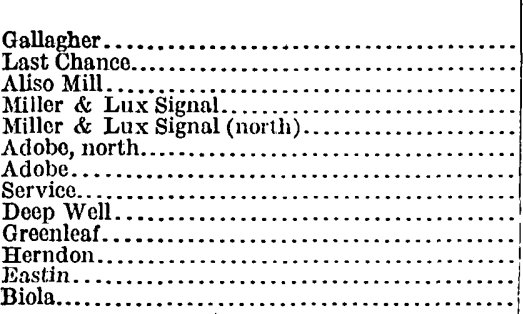 & $\begin{array}{rcc}\circ & \prime & \prime \prime \\
16 & 00 & 35.41 \\
37 & 29 & 32.66 \\
57 & 53 & 22.54 \\
73 & 33 & 13.19 \\
73 & 32 & 58.29 \\
245 & 06 & 23.10 \\
245 & 06 & 21.16 \\
266 & 20 & 00.20 \\
283 & 59 & 37.46 \\
308 & 10 & 25.10 \\
315 & 58 & 51.57 \\
336 & 03 & 20.02 \\
346 & 54 & 22.35\end{array}$ & \begin{tabular}{rcc}
\multicolumn{1}{c}{} & $\prime$ & $\prime \prime$ \\
195 & 58 & 38.45 \\
217 & 26 & 10.08 \\
237 & 46 & 21.25 \\
253 & 29 & 17.69 \\
253 & 29 & 02.77 \\
65 & 13 & 35.53 \\
65 & 13 & 33.55 \\
86 & 25 & 03.20 \\
104 & 07 & 08.92 \\
128 & 14 & 55.34 \\
136 & 04 & 07.65 \\
156 & 05 & 12.00 \\
166 & 56 & 02.01
\end{tabular} & $\begin{array}{r}\text { Log. meters. } \\
4.2433982 \\
4.1376429 \\
4.3120667 \\
4.0047251 \\
4.0047962 \\
4.2917831 \\
4.2917561 \\
4.0965713 \\
4.2824698 \\
4.1514200 \\
4.2734974 \\
4.0561051 \\
4.2594110\end{array}$ & $\begin{array}{r}\text { Miles. } \\
10.883 \\
8.531 \\
12.747 \\
6.282 \\
6.283 \\
12.166 \\
12.165 \\
7.761 \\
11.907 \\
8.806 \\
11.664 \\
7.071 \\
11.292\end{array}$ \\
\hline
\end{tabular}

MADERA, MADERA COUNTY, CALIF., FROM NORTH.

[Latitude $36^{\circ} 57^{\prime} 36.402^{\prime \prime}$ (from north), $36^{\circ} 57^{\prime} 36.362^{\prime \prime}$ (from south. Longitude $120^{\circ} 03^{\prime} 39.421^{\prime \prime}$ (from north), $120^{\circ} 03^{\prime} 39.663^{\prime \prime}$ ([rom south).]

\begin{tabular}{|c|c|c|c|c|}
\hline To station- & Azimuth. & Back azimuth. & Distar & ce. \\
\hline 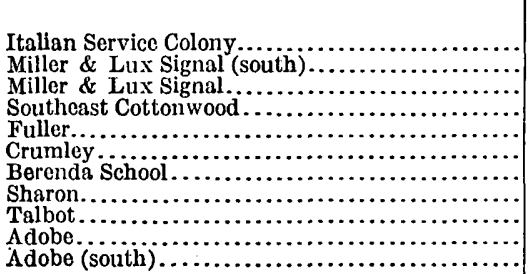 & $\begin{array}{rcc}\circ & \prime & \prime \prime \\
52 & 36 & 13.01 \\
73 & 33 & 13.19 \\
73 & 32 & 58.29 \\
82 & 12 & 56.92 \\
105 & 56 & 02.64 \\
124 & 01 & 29.82 \\
136 & 33 & 57.29 \\
158 & 14 & 42.44 \\
199 & 22 & 28.17 \\
245 & 06 & 23.10 \\
245 & 06 & 21.16\end{array}$ & $\begin{array}{ccc}\circ & \prime & \prime \prime \\
232 & 34 & 34.25 \\
253 & 29 & 17.69 \\
253 & 29 & 02.77 \\
262 & 06 & 02.76 \\
285 & 49 & 32.04 \\
303 & 57 & 40.42 \\
316 & 30 & 38.35 \\
338 & 12 & 10.96 \\
19 & 23 & 39.03 \\
65 & 13 & 35.53 \\
65 & 13 & 33.55\end{array}$ & $\begin{array}{r}\text { Log. meters. } \\
\text { 3. } 7091331 \\
\text { 4. } 0047251 \\
\text { 4. } 0047962 \\
\text { 4. } 2357339 \\
\text { 4. } 2226242 \\
\text { 4. } 0558576 \\
\text { 4. } 0749278 \\
\text { 4. } 2242262 \\
\text { 3. } 9431201 \\
\text { 4. } 2917831 \\
4.2917561\end{array}$ & $\begin{array}{r}\text { Miles. } \\
3.180 \\
6.282 \\
6.283 \\
10.693 \\
10.375 \\
7.067 \\
7.384 \\
10.413 \\
5.451 \\
12.166 \\
12.165\end{array}$ \\
\hline
\end{tabular}

MARGUERITE, MADERA COUNTY, CALIF.

Station is on south bank of Chowchilla River, half a mile south of Marguerite, a station on the Atchison, Topeka \& Santa Fe Ry., 300 feet west of the railroad and just south of the railroad bridge over Chowchilla River.

Signal: Quadripod with cross targets.

Station mark: Iron bench-mark post centered under signal.

[Latitude $37^{\circ} 09^{\prime} 41.096^{\prime \prime}$. Longitude $120^{\circ} 10^{\prime} 26.680^{\prime \prime}$.]

\begin{tabular}{|c|c|c|c|c|}
\hline Tostation- & Azimuth. & Back azimuth. & Dista & ce. \\
\hline 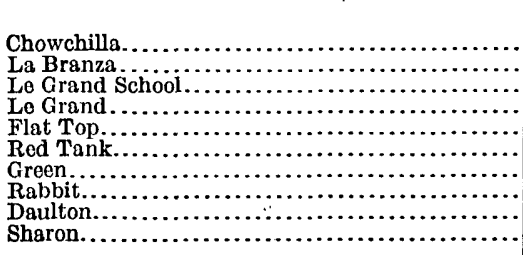 & $\begin{array}{rcc}\circ & \prime & \prime \prime \\
63 & 08 & 28.61 \\
97 & 10 & 59.06 \\
137 & 26 & 34.69 \\
153 & 18 & 48.04 \\
204 & 47 & 10.49 \\
208 & 51 & 57.08 \\
234 & 34 & 34.53 \\
277 & 07 & 25.24 \\
280 & 45 & 23.21 \\
330 & 25 & 36.57\end{array}$ & \begin{tabular}{rcc}
\multicolumn{1}{c}{$\circ$} & $\prime$ & $\prime \prime$ \\
243 & 05 & 19.97 \\
277 & 05 & 38.60 \\
317 & 23 & 45.09 \\
333 & 16 & 20.65 \\
24 & 49 & 29.32 \\
28 & 53 & 04.35 \\
54 & 41 & 14.52 \\
97 & 16 & 14.45 \\
100 & 52 & 19.07 \\
150 & 27 & 10.57
\end{tabular} & $\begin{array}{r}\text { Log. meters. } \\
\text { 3. } 9367548 \\
4.1201998 \\
4.0097013 \\
4.1261244 \\
4.1299824 \\
\text { 3. } 7547222 \\
4.3009786 \\
4.3384076 \\
4.2381142 \\
\text { 3. } 8916362\end{array}$ & $\begin{array}{r}\text { Miles. } \\
5.372 \\
8.195 \\
6.354 \\
8.308 \\
8.382 \\
3.532 \\
12.426 \\
13.544 \\
10.751 \\
4.842\end{array}$ \\
\hline
\end{tabular}

\section{MARTIN, SAN BENITO COUNTY, CALIF.}

On a low mountain, on which are scattered oaks, about 2 miles southwest of San Benito, probably on Mr. Martin's land but very near the Cornwall ranch. From San Benito take the King City road for about 1 mile, then turn to right up grade on road going to the Cornwall ranch. Just before reaching the Cornwall residence take lefthand road, passing an old barn, thence go west through gate and up rather steep hill. 
On summit of hill leave road and go north up ridge. It is possible to drive within 0.25 mile of signal.

Signal: A large oak on summit was trimmed and stump about 15 feet high was left for signal. Other high trees were cut.

Station mark: A tablet with a cross on it, set in rock placed in ground. This rock had been marked by E. P. Davis and was moved about 15 feet nearer the oak tree and reset. Position of mark computed. Azimuth from tablet to signal, $181^{\circ} 47^{\prime}$; distance, 1.87 meters.

[Latitude $36^{\circ} 29^{\prime} 10.534^{\prime \prime}$. Longitude $121^{\circ} 05^{\prime} 59.218^{\prime \prime}$.]

\begin{tabular}{|c|c|c|c|c|}
\hline To station- & Azimuth. & Back azimuth. & Dista & \\
\hline 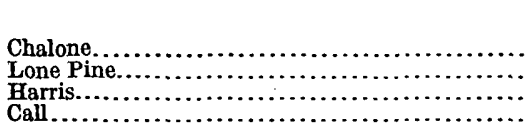 & $\begin{array}{rcc}\circ & \prime & \prime \prime \\
63 & 36 & 18.71 \\
122 & 25 & 44.54 \\
124 & 07 & 37.33 \\
193 & 20 & 01.54\end{array}$ & \begin{tabular}{rcc}
\multicolumn{1}{c}{} & $\prime$ & $\prime \prime$ \\
243 & 32 & 55.08 \\
302 & 23 & 17.73 \\
304 & 03 & 17.92 \\
13 & 21 & 19.43
\end{tabular} & \begin{tabular}{|r|} 
Log. meters. \\
3.9788655 \\
3.8618133 \\
4.1171623 \\
4.1490672
\end{tabular} & $\begin{array}{r}\text { Miles. } \\
5.919 \\
4.520 \\
8.138 \\
8.758\end{array}$ \\
\hline
\end{tabular}

MECO, SAN BENITO COUNTY, CALIF.

On a rather high peak, locally known as Meco Peak, 18 or 19 miles a little south of east from Tres Pinos. From Tres Pinos take the road going east to the Ingart ranch, thence by the Buena Vista ranch, thence to the Los Martin ranch. The peak is about 2 miles east of Los Martin ranch, and there is a trail from the ranch to the summit, which is on a school section just east of the ranch line. Top of peak is bald and has two or three large rocks on southeast side. There are several large oak trees on north side of peak.

Signal: An oak tree set 3 feet in the ground.

Station mark: A tablet cemented in rock. Position of tablet computed. Azimuth from tablet to signal, $74^{\circ} 10^{\prime}$; distance, 1.80 meters.

[Latitude $36^{\circ} 45^{\prime} 10.078^{\prime \prime}$. Longitude $121^{\circ} 03^{\prime} 25.835^{\prime \prime}$.]

\begin{tabular}{|c|c|c|c|c|}
\hline To station- & Azimuth. & Back azimuth. & Dista & \\
\hline 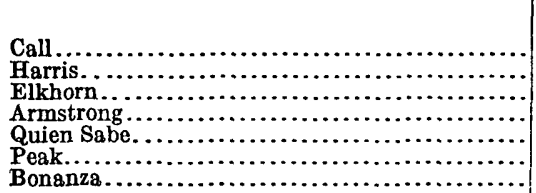 & $\begin{array}{rcc}\circ & \prime & \prime \prime \\
2 & 01 & 34.25 \\
33 & 24 & 13.23 \\
48 & 58 & 49.34 \\
55 & 41 & 53.53 \\
120 & 04 & 24.92 \\
137 & 31 & 03.27 \\
229 & 12 & 26.10\end{array}$ & \begin{tabular}{rcc}
\multicolumn{1}{c}{} & \multicolumn{1}{c}{} & $\prime \prime$ \\
182 & 01 & 20.75 \\
213 & 18 & 21.43 \\
228 & 55 & 45.05 \\
235 & 32 & 41.24 \\
299 & 58 & 37.17 \\
317 & 28 & 13.52 \\
49 & 15 & 11.38
\end{tabular} & $\begin{array}{r}\text { Log. meters. } \\
4.2006530 \\
4.4252340 \\
4.0060857 \\
4.4442413 \\
4.2208393 \\
4.0171081 \\
3.9561009\end{array}$ & $\begin{array}{r}\text { Miles. } \\
9.863 \\
16.542 \\
6.301 \\
17.282 \\
10.332 \\
6.463 \\
5.616\end{array}$ \\
\hline
\end{tabular}

MERCED, MERCED COUNTY, CALIF.

Station is Merced County courthouse at Merced.

Signal and station mark: Center of cupola.

[Latitude $37^{\circ} 18^{\prime} 24.733^{\prime \prime}$. Longitude $120^{\circ} 29^{\prime} 00.061^{\prime \prime}$.]

\begin{tabular}{|c|c|c|c|c|}
\hline To station- & Azimuth. & Back azimuth. & Diste & ce. \\
\hline 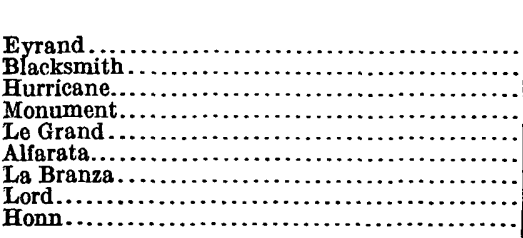 & $\begin{array}{ccc}\circ & \prime & \prime \prime \\
35 & 14 & 20.54 \\
217 & 58 & 17.90 \\
247 & 59 & 31.11 \\
277 & 29 & 44.07 \\
281 & 01 & 00.76 \\
309 & 24 & 02.88 \\
315 & 13 & 09.23 \\
346 & 05 & 30.06 \\
352 & 09 & 07.90\end{array}$ & \begin{tabular}{rcc}
\multicolumn{1}{c}{} & \multicolumn{1}{c}{} & \multicolumn{1}{c}{} \\
215 & 11 & 47.93 \\
38 & 01 & 52.87 \\
68 & 04 & 59.67 \\
97 & 33 & 34.19 \\
101 & 09 & 47.63 \\
129 & 25 & 41.83 \\
135 & 19 & 02.02 \\
166 & 06 & 51.56 \\
172 & 10 & 09.96
\end{tabular} & $\begin{array}{r}\text { Log. meters. } \\
4.0321314 \\
4.1511041 \\
4.1578254 \\
3.9746697 \\
4.3390540 \\
3.7165755 \\
4.3099650 \\
4.1404688 \\
4.2681636\end{array}$ & $\begin{array}{r}\text { Miles. } \\
6.691 \\
8.799 \\
8.937 \\
5.862 \\
13.565 \\
3.235 \\
12.686 \\
8.587 \\
11.522\end{array}$ \\
\hline
\end{tabular}


MILL, FRESNO COUNTY, CALIF. (Not occupied.)

Station mark: Center of windmill at farmhouse 2 miles northwest of Round triangulation station.

[Latitude $36^{\circ} 51^{\prime} 08.858^{\prime \prime}$. Longitude $119^{\circ} 31^{\prime} 21.038^{\prime \prime}$.]

\begin{tabular}{|c|c|c|c|c|}
\hline To station- & Azimuth. & Back azimuth. & Dista & \\
\hline 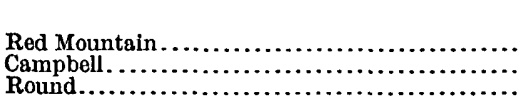 & $\begin{array}{ccc}\circ & \prime & \prime \prime \\
263 & 45 & 37.99 \\
332 & 26 & 27.92 \\
342 & 36 & 39.58\end{array}$ & $\begin{array}{rcc}\circ & \prime & \prime \prime \\
83 & 50 & 04.54 \\
152 & 30 & 06.56 \\
162 & 37 & 07.54\end{array}$ & \begin{tabular}{|r|} 
Log. meters. \\
4. 0442681 \\
4. 2922609 \\
3.5881341
\end{tabular} & $\begin{array}{r}\text { Miles. } \\
6.880 \\
12.179 \\
2.407\end{array}$ \\
\hline
\end{tabular}

\section{MILLER NO. 2, FRESNO COUNTY, CALIF.}

Center of windmill derrick at the Miller \& Lux Co.'s sheep ranch, about 0.75 mile southeast of the Sullivan ranch (which is also owned by the Miller \& Lux Co.). It is about 8 or 9 miles south of South D.os Palos, a station on the Southern Pacific R. R., on the west side of San Joaquin River. There is a barn and a very small cabin near windmill.

Station mark: The pipe extending into ground.

[Latitude $36^{\circ} 50^{\prime} 31.536^{\prime \prime}$. Longitude $120^{\circ} 38^{\prime} 45.218^{\prime \prime}$.]

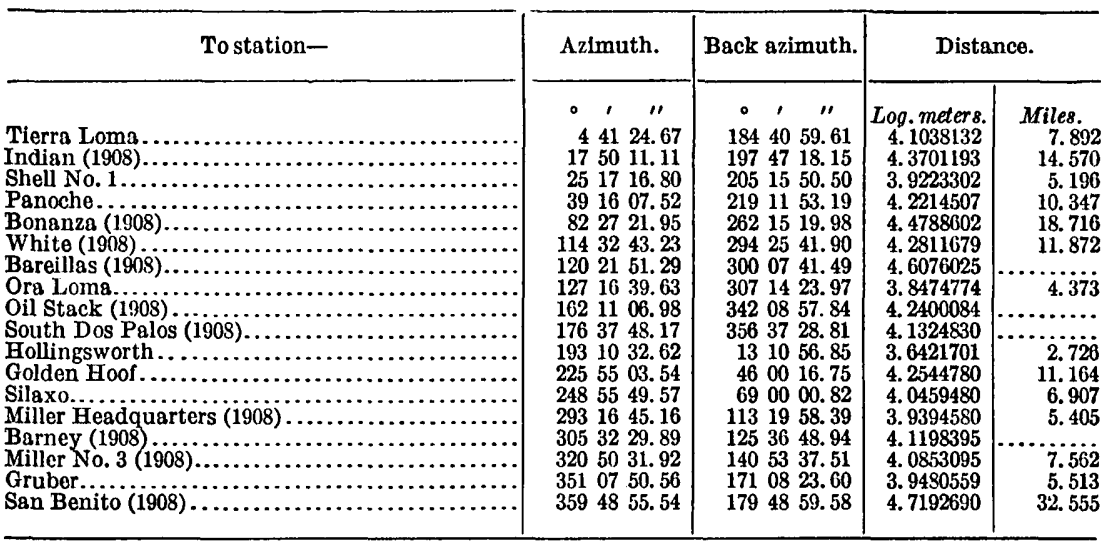

MILLER NO. 3, FRESNO COUNTY, CALIF.

The windmill derrick at the Miller \& Lux Co.'s sheep camp, known as the Whistler ranch, 3.5 or 4 miles south of the Headquarters sheep ranch, which is about 8 or 9 miles southwest of Firebaugh and about 10 or 11 miles west of Mendota, towns on the Southern Pacific R. R. A barn and two small white cabins are near the windmill, but there are no trees.

Station mark: The pipe extending into ground.

[Latitude $36^{\circ} 45^{\prime} 25.275^{\prime \prime}$. Longitude $120^{\circ} 33^{\prime} 35.388^{\prime \prime}$.]

\begin{tabular}{|c|c|c|c|c|}
\hline Tostation- & Azimuth. & Back azimuth. & Dista & ace. \\
\hline 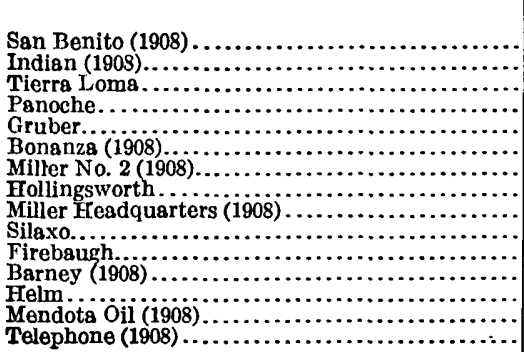 & \begin{tabular}{rcc}
\multicolumn{1}{c}{} & \multicolumn{1}{c}{} & \multicolumn{1}{c}{} \\
9 & 58 & 33.22 \\
49 & 08 & 18.84 \\
69 & 47 & 18.14 \\
79 & 19 & 10.54 \\
96 & 06 & 39.79 \\
98 & 21 & 35.12 \\
140 & 53 & 37.51 \\
154 & 03 & 17.32 \\
182 & 57 & 57.42 \\
191 & 21 & 55.80 \\
218 & 36 & 06.91 \\
239 & 43 & 19.21 \\
254 & 19 & 42.12 \\
261 & 28 & 56.92 \\
330 & 40 & 42.19
\end{tabular} & \begin{tabular}{rcc}
\multicolumn{1}{c}{} & $\prime$ & $\prime \prime$ \\
189 & 55 & 32.69 \\
229 & 02 & 20.90 \\
249 & 43 & 47.76 \\
259 & 11 & 51.12 \\
276 & 04 & 07.38 \\
278 & 06 & 28.35 \\
320 & 50 & 31.92 \\
334 & 00 & 35.85 \\
2 & 58 & 04.93 \\
11 & 23 & 01.13 \\
38 & 39 & 47.79 \\
59 & 44 & 32.56 \\
74 & 26 & 16.42 \\
81 & 34 & 45.86 \\
150 & 42 & 19.89
\end{tabular} & $\begin{array}{r}\text { Log. meters. } \\
\text { 4. } 6395952 \\
4.2938853 \\
3.9684437 \\
4.2683266 \\
3.8029572 \\
4.5791323 \\
4.0853095 \\
4.1833237 \\
3.7786616 \\
4.1367072 \\
4.1654803 \\
3.5464257 \\
4.2293593 \\
4.1648756 \\
3.9181290\end{array}$ & $\begin{array}{r}\text { Miles. } \\
\text { 12. } 225 \\
5.778 \\
11.526 \\
3.947 \\
7.562 \\
9.477 \\
3.733 \\
8.513 \\
9.096 \\
10.537\end{array}$ \\
\hline
\end{tabular}


MILLER HEADQUARTERS, FRESNO COUNTY, CALIF.

The windmill derrick at the Miller \& Lux Co.'s Headquarters sheep ranch. It is about 7 miles south of the superintendent's ranch, which is about halfway between South Dos Palos and Firebaugh, stations west of San Joaquin River on the Southern Pacific R. R.

Station mark: The iron pipe extending into ground.

[Latitude $36^{\circ} 48^{\prime} 39.888^{\prime \prime}$. Longitude $120^{\circ} 33^{\prime} 22.848^{\prime \prime}$.]

\begin{tabular}{|c|c|c|c|c|}
\hline Tostation- & Azimuth. & Back azimuth. & Distar & ce. \\
\hline 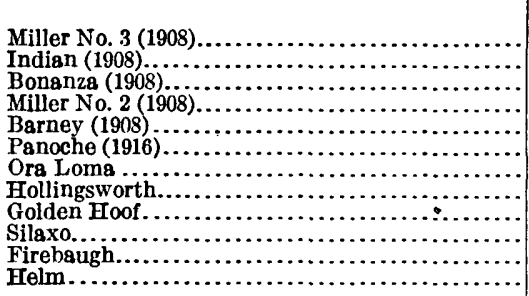 & \begin{tabular}{rcc}
$\bullet$ & \multicolumn{1}{c}{$\prime \prime$} \\
2 & 58 & 04.93 \\
38 & 50 & 06.50 \\
89 & 16 & 18.47 \\
113 & 19 & 58.39 \\
327 & 08 & 56.09 \\
63 & 01 & 29.01 \\
119 & 35 & 24.12 \\
137 & 51 & 10.68 \\
197 & 11 & 45.99 \\
197 & 49 & 17.60 \\
238 & 20 & 22.21 \\
275 & 03 & 46.83
\end{tabular} & \begin{tabular}{rcc|}
$\circ$ & $\prime$ & $\prime \prime$ \\
182 & 57 & 57.42 \\
218 & 44 & 00.83 \\
269 & 01 & 03.61 \\
293 & 16 & 45.16 \\
147 & 10 & 01.97 \\
242 & 54 & 01.81 \\
299 & 29 & 55.19 \\
317 & 48 & 21.59 \\
17 & 13 & 45.61 \\
17 & 50 & 15.45 \\
58 & 23 & 55.72 \\
95 & 10 & 13.86
\end{tabular} & \begin{tabular}{r|} 
Log. metcrs. \\
3. 7786616 \\
4. 3842510 \\
4. 5781693 \\
3. 9394580 \\
3. 7014854 \\
4. 3181120 \\
4.1937161 \\
4. 0173219 \\
4. 2221530 \\
3. 8924525 \\
4. 0155529 \\
4. 2062559
\end{tabular} & $\begin{array}{r}\text { Miles. } \\
3.733 \\
15.052 \\
23.521 \\
5.405 \\
\ldots .2 . \\
12.926 \\
9.707 \\
6.467 \\
10.363 \\
4.851 \\
6.440 \\
9.991\end{array}$ \\
\hline
\end{tabular}

MILEPOST 162, MERCED COUNTY, CALIF.

On bank of a canal, $5 \frac{1}{2}$ miles northwest of Los Banos, $2 \frac{1}{2}$ miles northwest of a Standard Oil Co.'s pumping station, and 20 feet north of milepost 162 . It can be reached by taking the north and south road from Los Banos to the Standard Oil Co.'s pumping station, thence going northwesterly along canal to station.

Signal and station mark: A 6 by 6 inch pole bearing cross targets.

Reference mark: Iron bench-mark post set on northwest side of pole.

Position of signal computed.

Azimuth from bench mark to signal, $139^{\circ} 15^{\prime}$; distance, 4.1 feet.

[Latitude $37^{\circ} 07^{\prime} 53.317^{\prime \prime}$. Longitude $121^{\circ} 51^{\prime} 36.818^{\prime \prime}$.]

\begin{tabular}{|c|c|c|c|c|}
\hline To station- & Azimuth. & Back azimuth. & Distar & ce. \\
\hline 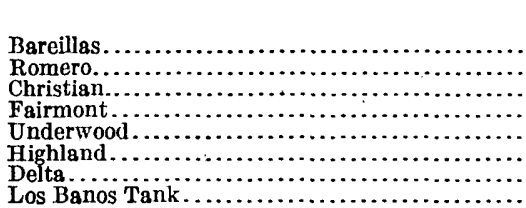 & \begin{tabular}{rcc}
\multicolumn{1}{c}{} & $\prime$ & $\prime \prime$ \\
53 & 38 & 41.10 \\
89 & 33 & 29.43 \\
108 & 23 & 12.41 \\
173 & 35 & 38.69 \\
195 & 46 & 53.21 \\
246 & 48 & 53.96 \\
278 & 11 & 00.31 \\
350 & 19 & 45.97
\end{tabular} & \begin{tabular}{rcc}
\multicolumn{1}{c}{} & $\prime$ & $\prime \prime$ \\
233 & 32 & 13.65 \\
269 & 27 & 52.77 \\
288 & 14 & 43.19 \\
353 & 35 & 07.72 \\
15 & 47 & 39.92 \\
66 & 52 & 18.43 \\
98 & 15 & 23.27 \\
170 & 20 & 18.55
\end{tabular} & $\begin{array}{r}\text { Log. meters. } \\
4.2949810 \\
4.1388420 \\
4.3397688 \\
4.0539241 \\
3.8459179 \\
3.9584686 \\
4.0360915 \\
3.9000410\end{array}$ & $\begin{array}{r}\text { Miles. } \\
12.255 \\
8.554 \\
13.587 \\
7.035 \\
4.358 \\
5.647 \\
6.752 \\
4.936\end{array}$ \\
\hline
\end{tabular}

MILLER \& LUX SIGNAL FROM SOUTH, MADERA COUNTY, CALFF.

In a Miller \& Lux pasture field, 7 miles a little south of west of Madera, on the Firebaugh road. The signal is 0.5 mile north of road and 0.5 mile south of a red tank and can easily be seen from road.

Signal: Quadripod with cross targets.

Station mark: Iron bench-mark post centered under signal.

[Latitude $36^{\circ} 56^{\prime} 03.334^{\prime \prime}$ (south), $36^{\circ} 56^{\prime} 03.338^{\prime \prime}$ (north). Longitude $120^{\circ} 10^{\prime} 11.453^{\prime \prime}$ (south), $120^{\circ} 10^{\prime}$ $11.267^{\prime \prime}$ (north).

\begin{tabular}{|c|c|c|c|c|}
\hline To station- & Azimuth. & Back azimuth. & Dista & ce. \\
\hline 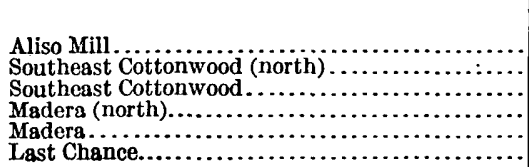 & \begin{tabular}{rcc}
\multicolumn{1}{c}{} & ' & " \\
43 & 36 & 59.63 \\
94 & 04 & 40.04 \\
94 & 04 & 48.78 \\
253 & 29 & 02.77 \\
253 & 29 & 17.69 \\
350 & 27 & 56.53
\end{tabular} & \begin{tabular}{rcc}
\multicolumn{1}{c}{} & $\prime$ & $\prime \prime$ \\
223 & 33 & 53.69 \\
274 & 01 & 41.48 \\
274 & 01 & 50.24 \\
72 & 32 & 58.29 \\
73 & 33 & 13.19 \\
170 & 28 & 29.22
\end{tabular} & $\begin{array}{r}\text { Log. meters. } \\
\text { 4. } 0461435 \\
\text { 3. } 8675666 \\
\text { 3. } 8675076 \\
\text { 4. } 0047962 \\
\text { 4. } 0047251 \\
\text { 3. } 9107280\end{array}$ & $\begin{array}{r}\text { Miles. } \\
6.910 \\
4.581 \\
4.580 \\
6.283 \\
6.282 \\
5.059\end{array}$ \\
\hline
\end{tabular}


MILIER \& IUX SIGNAL FROM NORTH, MADERA COUNTY, CALIF.

[Latitude $36^{\circ} 56^{\prime} 03.338^{\prime \prime}$ (north), $36^{\circ} 56^{\prime} 03.334^{\prime \prime}$ (South). Longitude $120^{\circ} 10^{\prime} 11.267^{\prime \prime}$ (north), $120^{\circ} 10^{\prime}$ $11.453^{\prime \prime}$ (south).

\begin{tabular}{|c|c|c|c|c|}
\hline Tostation- & Azimuth. & Back azimuth. & Dista1 & \\
\hline 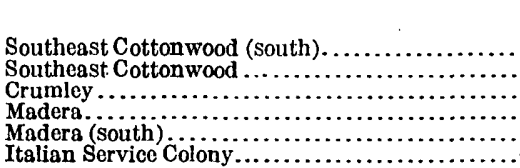 & \begin{tabular}{rcc|}
\multicolumn{1}{c}{} & $\prime$ & $\prime \prime$ \\
94 & 04 & 48.78 \\
94 & 04 & 40.04 \\
181 & 37 & 15.43 \\
253 & 29 & 02.77 \\
253 & 29 & 17.69 \\
272 & 25 & 41.38
\end{tabular} & \begin{tabular}{rcc|}
\multicolumn{1}{c}{} & $\prime$ & $\prime \prime$ \\
274 & 01 & 50.24 \\
274 & 01 & 41.48 \\
1 & 37 & 21.78 \\
73 & 32 & 58.29 \\
73 & 33 & 13.19 \\
92 & 27 & 58.10
\end{tabular} & \begin{tabular}{|r|} 
Log. meters. \\
3. 8675076 \\
3. 8675666 \\
3. 9652420 \\
4. 0047962 \\
4. 0047251 \\
3. 7509843
\end{tabular} & $\begin{array}{r}\text { Miles. } \\
4.580 \\
4.581 \\
5.736 \\
6.283 \\
6.282 \\
3.502\end{array}$ \\
\hline
\end{tabular}

MIIL TANK, MADERA COUNTY, CALIF. (Not occupled.)

Station mark: Windmill on tank house 6 or 8 miles northwest of Kermon.

[Latitude $36^{\circ} 48^{\prime} 00.837^{\prime \prime}$. Longitude $120^{\circ} 11^{\prime} 12.436^{\prime \prime}$.]

\begin{tabular}{|c|c|c|c|c|}
\hline Tostation- & Azimuth. & Back azimuth. & Dista & ce. \\
\hline 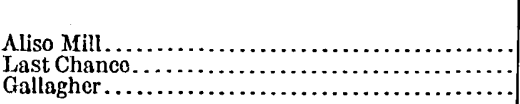 & $\begin{array}{ccc}\circ & \prime & \prime \prime \\
137 & 54 & 34.75 \\
-202 & 40 & 22.54 \\
261 & 55 & 34.88\end{array}$ & $\begin{array}{rcc}\circ & \prime & \prime \prime \\
317 & 52 & 05.66 \\
22 & 41 & 31.73 \\
81 & 58 & 09.38\end{array}$ & \begin{tabular}{r|} 
Log. meters. \\
3. 9633800 \\
3. 8702547 \\
3. 8100167
\end{tabular} & $\begin{array}{r}\text { Miles. } \\
\text { 5. } 711 \\
4.609 \\
4.012\end{array}$ \\
\hline
\end{tabular}

NORTH FRESNO CHIMNEY, FRESNO COUNTY, CAIIF. (Not occupled.)

Water standpipe for North Fresno, about 5 miles north of Fresno courthouse.

[Latitude $36^{\circ} 48^{\prime} 17.531^{\prime \prime}$. Longitude $119^{\circ} 48^{\prime} 48.414^{\prime \prime}$.]

\begin{tabular}{|c|c|c|c|c|}
\hline Tostation- & Azimuth. & Back azimuth. & Dista & ce. \\
\hline 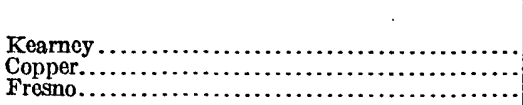 & \begin{tabular}{rcc}
\multicolumn{1}{c}{} &, & $\prime \prime$ \\
45 & 38 & 40.73 \\
226 & 30 & 01.10 \\
343 & 13 & 24.90
\end{tabular} & \begin{tabular}{rcc}
\multicolumn{1}{c}{} &, & $\prime \prime$ \\
225 & 35 & 02.97 \\
46 & 36 & 03.82 \\
163 & 14 & 19.68
\end{tabular} & $\begin{array}{r}\text { Log. meters. } \\
\text { 4. } 1169640 \\
\text { 4. } 3144082 \\
\text { 3. } 8957436\end{array}$ & $\begin{array}{r}\text { Miles. } \\
8.134 \\
12.816 \\
4.888\end{array}$ \\
\hline
\end{tabular}

OAK, FRESNO COUNTY, CALIF.

Station is a 14-inch oak tree on a high hill 6 miles north of academy. To reach it take the road running north and east from academy, keeping all left-hand roads for 6 miles to where the road passes under a lumber flume. Continue 200 feet to a stream crossing thence to left up second-class road around south base of hill. Follow this road 1 mile, more or less, to foot of hill. Animals can be ridden to top.

Signal and station mark: 14-inch oak tree.

Reference mark: A bronze tablet set in rock 35 feet north and 0.5 foot east of signal.

Position of signal computed.

[Latitude $36^{\circ} 57^{\prime} 21.371^{\prime \prime}$. Longitude $119^{\circ} 32^{\prime} 09.601^{\prime \prime}$.]

\begin{tabular}{|c|c|c|c|c|}
\hline Tostation- & Azimuth. & Back azimuth. & Distan & \\
\hline 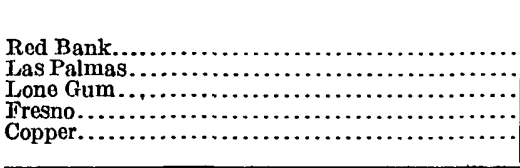 & $\begin{array}{ccc}\circ & \prime & \prime \prime \\
21 & 07 & 48.07 \\
33 & 17 & 04.21 \\
39 & 34 & 47.31 \\
42 & 51 & 06.41 \\
75 & 13 & 15.78\end{array}$ & $\begin{array}{ccc}\bullet & \prime & \prime \prime \\
201 & 05 & 29.94 \\
213 & 11 & 14.28 \\
219 & 31 & 07.71 \\
222 & 42 & 02.32 \\
255 & 09 & 18.82\end{array}$ & \begin{tabular}{|r|} 
Log. meters. \\
4.1992206 \\
4.4210865 \\
4.1528344 \\
4.5198238 \\
4.0039318
\end{tabular} & $\begin{array}{r}\text { Miles. } \\
9.830 \\
16.385 \\
8.835 \\
20.567 \\
6.270\end{array}$ \\
\hline
\end{tabular}


ORA LOMA, FRESNO COUNTY, CALIF.

Station is a steel tank 110 feet high 9 miles southwest of Dos Palos.

Signal and station mark: Center of tank.

[Latitude $36^{\circ} 52^{\prime} 49.771^{\prime \prime}$. Longitude $120^{\circ} 42^{\prime} 31.368^{\prime \prime}$.]

\begin{tabular}{|c|c|c|c|c|}
\hline Tostation- & Azimuth. & Back azimuth. & Dista & \\
\hline 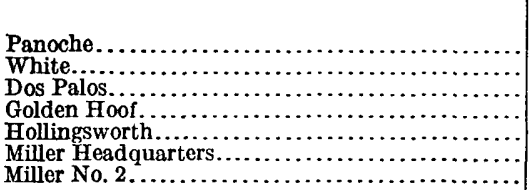 & \begin{tabular}{rcc}
\multicolumn{1}{c}{} &, & $\prime \prime$ \\
16 & 01 & 28.47 \\
107 & 17 & 06.15 \\
212 & 04 & 27.23 \\
245 & 58 & 15.37 \\
269 & 53 & 18.76 \\
299 & 29 & 55.19 \\
307 & 14 & 23.97
\end{tabular} & \begin{tabular}{rcc}
\multicolumn{1}{c}{} & $\prime$ & $\prime \prime$ \\
195 & 59 & 29.49 \\
287 & 12 & 20.40 \\
32 & 07 & 27.71 \\
66 & 05 & 44.56 \\
89 & 55 & 58.72 \\
119 & 35 & 24.12 \\
127 & 16 & 39.63
\end{tabular} & \begin{tabular}{r|} 
Log. meters. \\
4.2516671 \\
4.0912602 \\
4.1457490 \\
4.3065781 \\
3.8195797 \\
4.1937161 \\
3.8474774
\end{tabular} & $\begin{array}{r}\text { Miles. } \\
11.092 \\
7.667 \\
8.692 \\
12.587 \\
4.101 \\
9.707 \\
4.373\end{array}$ \\
\hline
\end{tabular}

ORANGE COVE, FRESNO COUNTY, CALIF. (Not occupied.)

Station mark: Center of tank.

[Latitude $36^{\circ} 37^{\prime} 23.138^{\prime \prime}$. Longitude $\left.119^{\circ} 18^{\prime} 38.728 . .^{\prime \prime}\right]$

\begin{tabular}{|c|c|c|c|c|}
\hline To station- & Azimuth. & Back azimuth. & Dista & \\
\hline 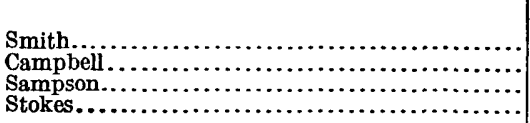 & $\begin{array}{rcc}\circ & \prime & \prime \prime \\
34 & 17 & 35.31 \\
129 & 19 & 56.83 \\
228 & 56 & 13.40 \\
318 & 58 & 27.37\end{array}$ & \begin{tabular}{rcc}
\multicolumn{1}{c}{} & \multicolumn{1}{c}{$l \prime$} \\
214 & 16 & 21.22 \\
309 & 15 & 59.79 \\
49 & 03 & 42.86 \\
139 & 02 & 30.03
\end{tabular} & \begin{tabular}{|r|} 
Log. meters. \\
3.7389986 \\
4.1052375 \\
4.3934012 \\
4.1886054
\end{tabular} & $\begin{array}{r}\text { Miles. } \\
3.407 \\
7.917 \\
15.373 \\
9.593\end{array}$ \\
\hline
\end{tabular}

OROSI, FRESNO COUNTY, CALIF. (Not occupled.)

Station mark: Center of black steel tank.

[Latitude $36^{\circ} 32^{\prime} 56.977^{\prime \prime}$. Longitude $119^{\circ} 15^{\prime} 35.077^{\prime \prime}$.]

\begin{tabular}{|c|c|c|c|c|}
\hline Tostation- & Azimuth. & Back azimuth. & Dista1 & ce. \\
\hline 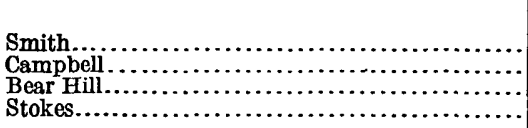 & $\begin{array}{ccc}\circ & \prime & \prime \prime \\
115 & 40 & 01.30 \\
138 & 29 & 30.91 \\
174 & 39 & 44.62 \\
301 & 45 & 58.30\end{array}$ & $\begin{array}{ccc}\circ & \prime & \prime \prime \\
295 & 36 & 57.87 \\
318 & 23 & 44.52 \\
354 & 38 & 55.52 \\
121 & 48 & 11.42\end{array}$ & \begin{tabular}{|c|} 
Log. meters. \\
3.9290562 \\
4.3374712 \\
4.3411858 \\
3.8158841
\end{tabular} & $\begin{array}{r}\text { Miles. } \\
5.277 \\
13.515 \\
13.631 \\
4.067\end{array}$ \\
\hline
\end{tabular}

PAICINES, SAN BENTTO COUNTY, GALIF.

On a bald ridge about 3 miles south of Tres Pinos. The ridge is between San Benito River and Tres Pinos Creek on land owned by a Mr. Balboa or Brown. He is a Portuguese and is known by both names. From Tres Pinos take the Paicines road until opposite Mr. Balboa's residence, then go to right up hill on farm road for 0.25 mile, thence on horseback to summit of hill.

Signal: An oak tree set 3.5 feet in the ground.

Station mark: A triangulation tablet set in a rock placed in ground. Position of tablet was computed. Azimuth from tablet to signal, $58^{\circ} 33^{\prime}$; distance, 1.38 meters.

[Latitude $36^{\circ} 45^{\prime} 19.066^{\prime \prime}$. Longitude $121^{\circ} 18^{\prime} 22.469^{\prime \prime}$.]

\begin{tabular}{|c|c|c|c|c|}
\hline Tostation- & Azimuth. & Back azimuth. & Distan & \\
\hline 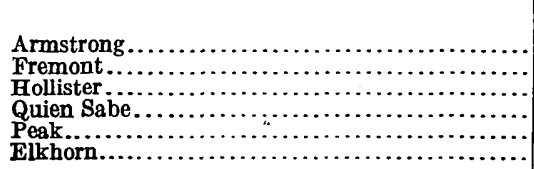 & \begin{tabular}{rcc}
\multicolumn{1}{c}{} & $\prime$ & $\prime \prime$ \\
2 & 28 & 56.92 \\
90 & 51 & 55.13 \\
141 & 10 & 54.17 \\
224 & 13 & 10.06 \\
244 & 01 & 19.58 \\
295 & 21 & 36.26
\end{tabular} & \begin{tabular}{rcc}
\multicolumn{1}{c}{} &, & $\prime \prime$ \\
182 & 28 & 40.26 \\
270 & 44 & 50.36 \\
321 & 07 & 24.50 \\
44 & 15 & 19.31 \\
64 & 07 & 26.79 \\
115 & 27 & 28.12
\end{tabular} & \begin{tabular}{|r|} 
Log. meters. \\
4.2040293 \\
4.2457265 \\
4.1408906 \\
4.0503057 \\
4.2280110 \\
4.2085915
\end{tabular} & $\begin{array}{r}\text { Miles. } \\
9.940 \\
10.942 \\
8.595 \\
6.977 \\
10.504 \\
10.045\end{array}$ \\
\hline
\end{tabular}


PANOChE MOUNTAIN, FRESNO COUNTY, CALIF.

A lumber tripod on bald hill, about 20 miles west of Mendota. It is about the highest point between the stations Indian and White and on line between them.

Station mark: Iron bench-mark post under center of station.

[Latitude $36^{\circ} 43^{\prime} 33.120^{\prime \prime}$. Longitude $120^{\circ} 45^{\prime} 49.954^{\prime \prime}$.]

\begin{tabular}{|c|c|c|c|c|}
\hline To station- & Azimuth. & Back azimuth. & Dista & \\
\hline 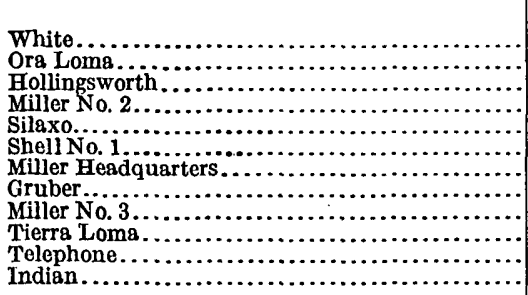 & $\begin{array}{ccc}\circ & \prime & \prime \prime \\
161 & 44 & 58.70 \\
195 & 59 & 29.49 \\
213 & 50 & 41.02 \\
219 & 11 & 53.19 \\
231 & 00 & 26.69 \\
232 & 30 & 56.09 \\
242 & 54 & 01.81 \\
250 & 49 & 26.18 \\
259 & 11 & 51.12 \\
268 & 31 & 17.18 \\
279 & 30 & 47.63 \\
340 & 20 & 00.00\end{array}$ & \begin{tabular}{rcc}
\multicolumn{1}{c}{} & $\prime$ & $\prime \prime$ \\
341 & 42 & 12.49 \\
16 & 01 & 28.47 \\
33 & 55 & 19.66 \\
39 & 16 & 07.52 \\
51 & 08 & 52.04 \\
52 & 33 & 44.04 \\
63 & 01 & 29.01 \\
70 & 54 & 13.27 \\
79 & 19 & 10.54 \\
88 & 35 & 06.15 \\
99 & 39 & 44.38 \\
160 & 21 & 21.01
\end{tabular} & \begin{tabular}{|r|} 
Log. meters. \\
4.3408881 \\
4.2516671 \\
4.3155823 \\
4.2214507 \\
4.4294245 \\
3.9431269 \\
4.3181120 \\
4.1004317 \\
4.2683266 \\
3.9779082 \\
4.3541563 \\
4.0004416
\end{tabular} & $\begin{array}{r}\text { Miles. } \\
13.622 \\
11.092 \\
12.851 \\
10.347 \\
16.702 \\
5.451 \\
12.926 \\
7.830 \\
11.526 \\
5.906 \\
14.045 \\
6.220\end{array}$ \\
\hline
\end{tabular}

PARLIER, FRESNO COUNTY, CALIF. (Not occupled.)

Station mark: Center of high, white wooden tank in Parlier.

[Latitude $36^{\circ} 36^{\prime} 37.278^{\prime \prime}$. Longitude $119^{\circ} 31 \cdot 41.655^{\prime \prime}$.]

\begin{tabular}{|c|c|c|c|c|}
\hline To station- & Azimuth. & Back azimuth. & Distan & ce. \\
\hline 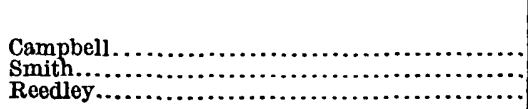 & $\begin{array}{lcc}\circ & \prime & \prime \prime \\
225 & 16 & 02.53 \\
280 & 43 & 08.05 \\
282 & 19 & 37.28\end{array}$ & $\begin{array}{rcc}4 & \prime \prime \\
45 & 19 & 52.90 \\
100 & 49 & 40.73 \\
102 & 22 & 21.16\end{array}$ & $\begin{array}{r}\text { Log. meters. } \\
4.1298555 \\
4.2218216 \\
3.8446330\end{array}$ & $\begin{array}{r}\text { Miles. } \\
8.379 \\
10.356 \\
4.345\end{array}$ \\
\hline
\end{tabular}

PEAK, SAN BENITO COUNTY, CAIIF. (Not occupied.)

Highest point of sharp peak about 5 miles east of Quien Sabe Peak.

[Latitude $36^{\circ} 49^{\prime} 18.844^{\prime \prime}$. Longitude $121^{\circ} 08^{\prime} 09.288^{\prime \prime}$.]

\begin{tabular}{|c|c|c|c|c|}
\hline Tostation- & Azimuth. & Back azimuth. & Dista1 & ce. \\
\hline 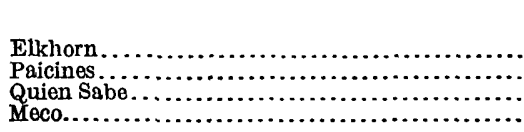 & $\begin{array}{rcc}\circ & \prime & \prime \prime \\
2 & 27 & 21.83 \\
64 & 07 & 26.79 \\
95 & 05 & 02.68 \\
317 & 28 & 13.52\end{array}$ & $\begin{array}{ccc}\circ & \prime & \prime \prime \\
182 & 27 & 07.02 \\
244 & 01 & 19.58 \\
275 & 02 & 04.55 \\
137 & 31 & 03.27\end{array}$ & $\begin{array}{r}\text { Log. meters. } \\
4.1565683 \\
4.2280110 \\
3.8688911 \\
4.0171081\end{array}$ & $\begin{array}{r}\text { Miles. } \\
8.911 \\
10.504 \\
4.595 \\
6.463\end{array}$ \\
\hline
\end{tabular}

PINE CREEK, MONTEREY COUNTY, CALIF. (Not occupied.)

On a brushy hill about 8 miles southwest of King City, a town on Southern Pacific R. R. Coast Line. Old road and trail to San Antonio Valley passes near signal.

[Latitude $36^{\circ} 06^{\prime} 12.077^{\prime \prime}$. Longitude $121^{\circ} 13^{\prime} 40.072^{\prime \prime}$.]

\begin{tabular}{|c|c|c|c|c|}
\hline Tostation- & Azimuth. & Back azimuth. & Distar & \\
\hline 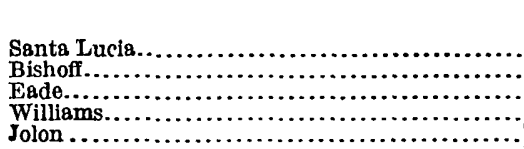 & $\begin{array}{ccc}\circ & \prime & \prime \prime \\
105 & 27 & 13.69 \\
194 & 27 & 02.84 \\
239 & 42 & 49.12 \\
309 & 21 & 44.89 \\
348 & 29 & 16.88\end{array}$ & \begin{tabular}{rcc}
\multicolumn{1}{c}{} & $\prime$ & $\prime \prime$ \\
285 & 20 & 29.35 \\
14 & 28 & 01.55 \\
59 & 51 & 58.55 \\
129 & 29 & 45.23 \\
168 & 30 & 18.46
\end{tabular} & \begin{tabular}{|r|} 
Log. meters. \\
4.2501683 \\
3.9985104 \\
4.4302126 \\
4.4227868 \\
4.1184733
\end{tabular} & $\begin{array}{r}\text { Miles. } \\
11.054 \\
6.192 \\
16.733 \\
16.449 \\
8.162\end{array}$ \\
\hline
\end{tabular}




\section{PIPE LINE, MERCED COUNTY, CALIF.}

On the oil pipe line, 6 miles southwest of Los Banos and 5 miles east of Bareillas triangulation station. The station is on the highest point of the pipe line and is about 400 feet due.west of a fence corner.

Signal: Lumber tripod.

Station mark: A rock about 1 foot in diameter buried at foot of signal.

[Latitude $37^{\circ} 01^{\prime} 40.460^{\prime \prime}$. Longitude $120^{\circ} 56^{\prime} 45.203^{\prime \prime}$.]

\begin{tabular}{|c|c|c|c|c|}
\hline To station- & Azimuth. & Back azimuth. & Distal & \\
\hline 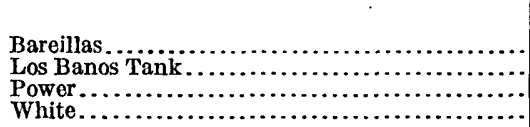 & $\begin{array}{rcc}\circ & \prime & \prime \prime \\
88 & 33 & 11.41 \\
247 & 43 & 30.34 \\
261 & 22 & 16.02 \\
323 & 36 & 59.72\end{array}$ & \begin{tabular}{rcc}
\multicolumn{1}{c}{} & $\prime$ & $\prime \prime$ \\
268 & 29 & 50.13 \\
67 & 47 & 08.65 \\
81 & 30 & 28.56 \\
143 & 40 & 46.98
\end{tabular} & \begin{tabular}{|r|} 
Log. meters. \\
3.9172138 \\
3.9856751 \\
4.3103626 \\
4.1978358
\end{tabular} & $\begin{array}{r}\text { Miles. } \\
5.135 \\
6.012 \\
12.697 \\
9.799\end{array}$ \\
\hline
\end{tabular}

PLANADA, MERCED COUNTY, CALIF. (Not occupied.)

Station mark: Center of a high water tank at Planada, a small town on the Atchison, Topeka \& Santa Fe R. R. about 10 miles east of Merced.

[Latitude $37^{\circ} 17^{\prime} 22.116^{\prime \prime}$. Longitude $120^{\circ} 19^{\prime} 24.522^{\prime \prime}$.]

\begin{tabular}{|c|c|c|c|c|}
\hline Tostation- & Azimuth. & Backazimuth. & Dista & \\
\hline 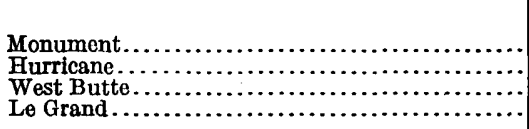 & \begin{tabular}{rcc}
\multicolumn{1}{c}{} & \multicolumn{1}{c}{$\quad$} & \multicolumn{1}{c}{} \\
98 & 12 & 41.59 \\
173 & 31 & 14.12 \\
208 & 49 & 36.32 \\
287 & 21 & 35.11
\end{tabular} & \begin{tabular}{rcc}
\multicolumn{1}{c}{} & \multicolumn{1}{c}{$\quad c$} \\
278 & 10 & 42.96 \\
353 & 30 & 53.67 \\
28 & 52 & 54.28 \\
107 & 24 & 33.27
\end{tabular} & $\begin{array}{r}\text { Log. meters. } \\
3.6877652 \\
3.8666605 \\
4.2211092 \\
3.8804168\end{array}$ & $\begin{array}{r}\text { Miles. } \\
3.028 \\
4.571 \\
10.339 \\
4.718\end{array}$ \\
\hline
\end{tabular}

POWELL, MONTEREY COUNTY, CALIF.

On the highest part of a round brushy.peak on north side of Powell Canyon and not very far from its head. The peak is about 2 miles from Mr. Powell's residence, which can be reached by road from Bradley or San Ardo, the best route being from Bradley. Animals can be ridden to summit of peak. Distance from Bradley to signal is about 14 miles. All brush was cut from the summit except one cluster.

Signal: A cluster of brush 8 or 9 feet high.

Station mark: A bench-mark tablet set in rock. Position of tablet was computed. Azimuth from tablet to signal, $89^{\circ} 47^{\prime}$; distance, 1.60 meters.

[Latitude $36^{\circ} 00^{\prime} 08.165^{\prime \prime}$. Longitude $120^{\circ} 43^{\prime} 01.025^{\prime \prime}$.]

\begin{tabular}{|c|c|c|c|c|}
\hline Tostation- & Azimuth. & Backazimuth. & DistaI & ce. \\
\hline 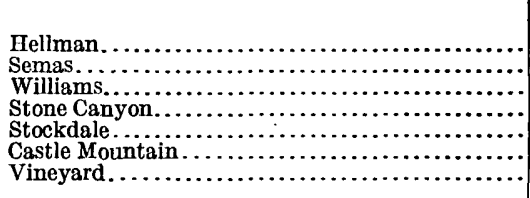 & $\begin{array}{ccc}\circ & \prime & \prime \prime \\
10 & 35 & 39.07 \\
37 & 08 & 35.71 \\
77 & 45 & 28.32 \\
260 & 12 & 53.80 \\
277 & 31 & 04.14 \\
281 & 29 & 56.41 \\
350 & 41 & 58.26\end{array}$ & \begin{tabular}{rcc}
\multicolumn{1}{c}{} & $\prime$ & $\prime \prime$ \\
190 & 33 & 50.48 \\
217 & 04 & 50.29 \\
257 & 35 & 27.71 \\
80 & 18 & 40.66 \\
97 & 35 & 52.05 \\
101 & 43 & 14.13 \\
170 & 43 & 30.24
\end{tabular} & $\begin{array}{r}\text { Log. meters. } \\
4.4031826 \\
4.2028012 \\
4.4186037 \\
4.1757593 \\
4.0926416 \\
4.5408143 \\
4.3870387\end{array}$ & $\begin{array}{r}\text { Miles. } \\
15.723 \\
9.912 \\
16.291 \\
9.313 \\
7.691 \\
21.586 \\
15.149\end{array}$ \\
\hline
\end{tabular}

POWER, MERCED COUNTY, CALIF.

A power pole on the north edge of the highway, 8 miles east of Los Banos. The pole has wire braces and stands on the south bank of the slough; it is the first pole west of four willow trees in the road.

Signal and station mark: A power pole 1 foot in diameter with cross targets.

Reference mark: Iron bench-mark post 3.2 feet north of center of pole.

Position of signal computed. 'Azimuth from tablet to signal, $6^{\circ} 49^{\prime}$; distance, 3.2 feet. 
[Latitude $37^{\circ} 03^{\prime} 19.132^{\prime \prime}$. Longitude $120^{\circ} 43^{\prime} 07.531^{\prime \prime}$.]

\begin{tabular}{|c|c|c|c|c|}
\hline To station- & Azimuth. & Backarimuth. & Dis & nce. \\
\hline 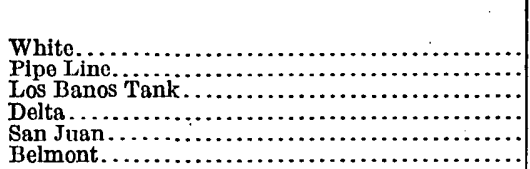 & $\begin{array}{rcc}\circ & . & \prime \prime \\
34 & 40 & 31.53 \\
81 & 30 & 28.56 \\
93 & 11 & 53.77 \\
165 & 14 & 30.62 \\
242 & 53 & 10.08 \\
283 & 09 & 48.67\end{array}$ & \begin{tabular}{rcc}
\multicolumn{1}{c}{} & $\prime$ & $\prime \prime$ \\
214 & 36 & 06.97 \\
261 & 22 & 16.02 \\
273 & 07 & 19.41 \\
345 & 13 & 46.24 \\
62 & 55 & 24.86 \\
103 & 13 & 15.90
\end{tabular} & $\begin{array}{r}\text { Log. meters. } \\
4.2818150 \\
4.3103626 \\
4.0517582 \\
3.8533472 \\
3.7927464 \\
3.9410284\end{array}$ & $\begin{array}{r}\text { Miles. } \\
11.890 \\
12.697 \\
7.000 \\
4.433 \\
3.856 \\
5.425\end{array}$ \\
\hline
\end{tabular}

QUIEN SABE, SAN BENITO COUNTY, CALIF.

On a sharp peak, locally known as the Quien Sabe Peak, about 7 miles by road east-northeast of Tres Pinos. From Tres Pinos take the road going east to Buena Vista, but turn to left just before crossing bridge at schoolhouse. This road goes up a long grade and crosses ridge and thence to Quien Sabe Valley. On summit of this ridge leave road and go through gate, thence northeast on horseback to summit of peak.

Signal: An oak tree set in the ground with a small pile of rock around its base.

Station mark: A triangulation tablet set in solid rock under center of signal.

[Latitude $36^{\circ} 49^{\prime} 39.997^{\prime \prime}$. Longitudo $121^{\circ} 13^{\prime} 06.487^{\prime \prime}$.]

\begin{tabular}{|c|c|c|c|c|}
\hline To station一 & Azimuth. & Back azimuth. & Dista & ce. \\
\hline 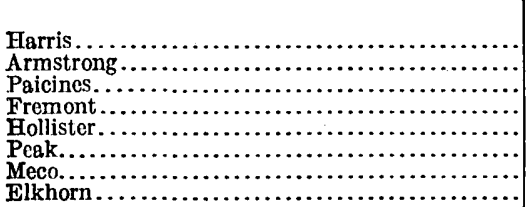 & $\begin{array}{rcc}\circ & \prime & \prime \prime \\
0 & 24 & 12.06 \\
19 & 34 & 59.63 \\
44 & 16 & 19.31 \\
73 & 02 & 39.82 \\
99 & 26 & 51.55 \\
275 & 02 & 04.55 \\
299 & 58 & 37.17 \\
335 & 41 & 46.65\end{array}$ & \begin{tabular}{rcc}
\multicolumn{1}{c}{} & $\prime$ & $\prime \prime$ \\
180 & 24 & 06.89 \\
199 & 31 & 34.03 \\
224 & 13 & 10.06 \\
252 & 52 & 25.44 \\
279 & 20 & 12.24 \\
95 & 05 & 02.68 \\
120 & 04 & 24.02 \\
155 & 44 & 29.70
\end{tabular} & $\begin{array}{r}\text { Log. meters. } \\
4.4851199 \\
4.4064630 \\
4.0503057 \\
4.4248932 \\
4.2233829 \\
3.8688911 \\
4.2208393 \\
4.2157235\end{array}$ & $\begin{array}{r}\text { Miles. } \\
18.988 \\
15.842 \\
6.977 \\
16.529 \\
10.393 \\
4.595 \\
10.332 \\
10.211\end{array}$ \\
\hline
\end{tabular}

RABBIT, MADERA COUNTY, CALIF.

On a bald hill about 5 miles from Raymond. The road from Raymond to Berenda passes within 0.5 mile of station at point where there is a water trough near gate. Go through gate and up the hill.

Signal and station mark: Cairn 7 feet high; triangulation tablet under center.

[Latitude $37^{\circ} 08^{\prime} 12.98^{\prime \prime}(1908), 37^{\circ} 08^{\prime} 12.509^{\prime \prime}(1915)$. Longitudo $119^{\circ} 55^{\prime} 49.51^{\prime \prime}(1908), 119^{\circ} 55^{\prime} 50.351^{\prime \prime}$

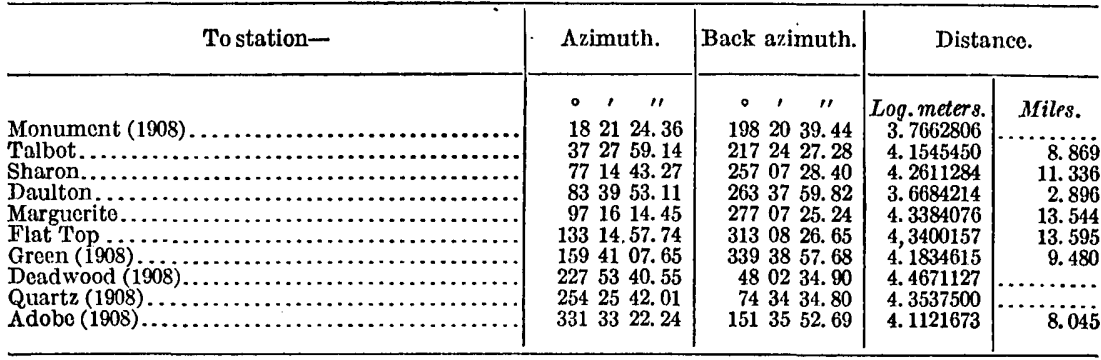

RED BANK, FRESNO COUNTY, CALIF.

Station is windmill at the Red Bank School, 7 miles east of Clovis.

Signal: Center of steel windmill tower.

Station mark: Pipe in well under center of tower. 
[Latitude $36^{\circ} 49^{\prime} 22.593^{\prime \prime}$. Longitude $119^{\circ} 35^{\prime} 59.716^{\prime \prime}$.

\begin{tabular}{|c|c|c|c|c|}
\hline Tostation- & Azimuth. & Back azimuth. & Dista & \\
\hline 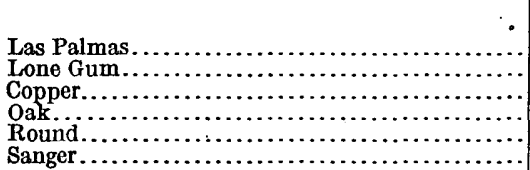 & $\begin{array}{rcc}\circ & \prime & \prime \prime \\
50 & 14 & 05.92 \\
138 & 30 & 21.20 \\
161 & 33 & 20.54 \\
201 & 05 & 29.94 \\
272 & 57 & 47.37 \\
342 & 52 & 24.06\end{array}$ & \begin{tabular}{rcc}
\multicolumn{1}{c}{$\circ$} & $\prime$ & $\prime \prime$ \\
230 & 10 & 34.34 \\
318 & 28 & 59.91 \\
341 & 31 & 42.04 \\
21 & 07 & 48.07 \\
93 & 01 & 02.37 \\
162 & 53 & 58.19
\end{tabular} & \begin{tabular}{|r|} 
Log. meters. \\
4. 0569118 \\
3. 7049268 \\
4. 1085492 \\
4.1992206 \\
3.9071625 \\
4.1224134
\end{tabular} & $\begin{array}{r}\text { Miles. } \\
7.084 \\
3.150 \\
7.978 \\
9.830 \\
5.018 \\
8.237\end{array}$ \\
\hline
\end{tabular}

RED MOUNTAIN, FRESHO COUNTY, CALIF.

On the north end of a long base ridge 15 miles northeast of Sanger. Follow Trimmer road from Sanger 15 miles to the mouth of Hugh's creek, thence go up Hugh's creek $2 \frac{1}{2}$ miles to a mine, thence west up a ridge just north of the mine to the summit, and thence north to signal, which is about 2 miles from the mine. Animals can be ridden to the top.

Signal: Cairn surrounding tree with white cloth. There is a large oak tree 100 feet north and a little east of cairn.

Station mark: Chisel mark under cairn.

Reference mark: Bronze tablet 6.2 feet north of center of cairn. Position of tree computed.

[Latitude $36^{\circ} 51^{\prime} 47.673^{\prime \prime}$. Longitude $11^{\circ} 23^{\prime} 56.663^{\prime \prime}$.]

\begin{tabular}{|c|c|c|c|c|}
\hline To station- & Azimuth. & Back azimuth. & Dista & ce. \\
\hline 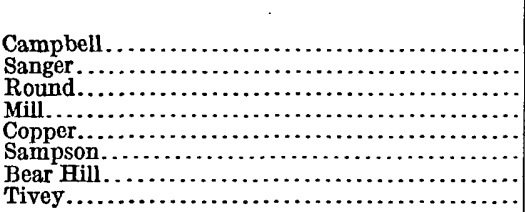 & $\begin{array}{rcc}- & \prime & \prime \prime \\
6 & 01 & 57.10 \\
39 & 20 & 38.57 \\
63 & 37 & 20.49 \\
83 & 50 & 04.54 \\
109 & 24 & 26.58 \\
271 & 21 & 12.27 \\
321 & 20 & 38.75 \\
358 & 31 & 06.83\end{array}$ & \begin{tabular}{ccc|}
$\circ$ & $\prime$ & $\prime \prime$ \\
186 & 01 & 09.75 \\
219 & 14 & 59.77 \\
243 & 33 & 22.06 \\
263 & 45 & 37.99 \\
289 & 15 & 33.91 \\
111 & 31 & 53.52 \\
141 & 24 & 49.96 \\
178 & 31 & 15.14
\end{tabular} & \begin{tabular}{r|} 
Log. meters. \\
4.2713925 \\
4.3453730 \\
4.0414461 \\
4.0442681 \\
4.3669627 \\
4.4547651 \\
4.2215142 \\
4.1244156
\end{tabular} & $\begin{array}{r}\text { Miles. } \\
11.608 \\
13.763 \\
6.836 \\
6.880 \\
14.465 \\
17.706 \\
10.348 \\
8.275\end{array}$ \\
\hline
\end{tabular}

RED ROCK, SAN LUIS OBISPO COUNTY, CALIF. (Not occupied.)

Summit of very sharp, rocky peak.

[Latitude $35^{\circ} 45^{\prime} 23.920^{\prime \prime}$. Longitude $121^{\circ} 09^{\prime} 34.769^{\prime \prime}$.]

\begin{tabular}{|c|c|c|c|c|}
\hline To station- & Azimuth. & Back azimuth. & Distar & \\
\hline 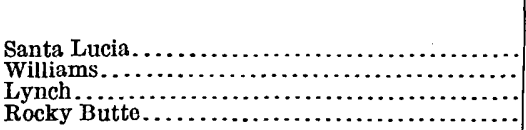 & $\begin{array}{ccc}\circ & \prime & \prime \prime \\
151 & 41 & 38.66 \\
213 & 27 & 31.69 \\
264 & 03 & 10.23 \\
317 & 54 & 48.42\end{array}$ & \begin{tabular}{rcc}
\multicolumn{1}{c}{} & \multicolumn{1}{c}{$\prime \prime$} \\
331 & 32 & 31.97 \\
33 & 33 & 06.35 \\
84 & 09 & 17.14 \\
137 & 58 & 20.14
\end{tabular} & \begin{tabular}{|r|} 
Log. meters. \\
4.6910257 \\
4.4145015 \\
4.2001510 \\
4.1338783
\end{tabular} & $\begin{array}{r}\text { Miles. } \\
30.505 \\
16.138 \\
9.851 \\
8.457\end{array}$ \\
\hline
\end{tabular}

RED TANE, MERCED COUNTY, CALIF. (Not occupied.)

A water tank painted red at ranch about 6 miles east-southeast of Le Grand, a small town on the Atchison, Topeka \& Santa Fe Ry.

Station mark: Center of tank.

[Latitude $37^{\circ} 12^{\prime} 22.580^{\prime \prime}$. Longitude $120^{\circ} 08^{\prime} 35.385^{\prime \prime}$.]

\begin{tabular}{|c|c|c|c|c|}
\hline To station- & Azimuth. & Back azimuth. & Dista & \\
\hline 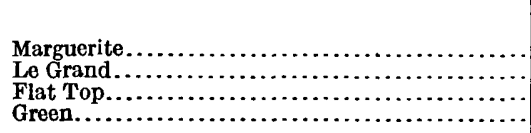 & $\begin{array}{rcc}\circ & , & \prime \prime \\
28 & 53 & 04.35 \\
128 & 32 & 59.79 \\
201 & 50 & 37.68 \\
244 & 00 & 30.31\end{array}$ & \begin{tabular}{rcc|}
\multicolumn{1}{c}{} & $\prime$ & $\prime \prime$ \\
208 & 51 & 57.08 \\
308 & 29 & 24.97 \\
21 & 51 & 49.23 \\
64 & 06 & 03.16
\end{tabular} & \begin{tabular}{r|} 
Loq. meters. \\
3. 7547222 \\
4. 0486003 \\
3. 8937089 \\
4. 1783613
\end{tabular} & $\begin{array}{l}\text { Miles. } \\
3.532 \\
6.950 \\
4.865 \\
9.369\end{array}$ \\
\hline
\end{tabular}


REEDLEY, FRESITO COUNTY, CALIF.

Municipal steel water tank at Reedley.

Signal and station mark: Center of tank.

[Latitude $36^{\circ} 35^{\prime} 48.759^{\prime \prime}$. Longitude $119^{\circ} 27^{\prime} 06.820^{\prime \prime}$.]

\begin{tabular}{|c|c|c|c|c|}
\hline Tostation- & Azimuth. & Back azimuth. & Distar & co. \\
\hline 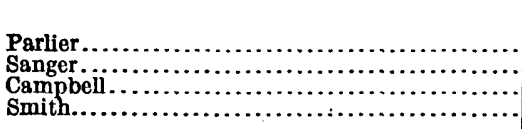 & $\begin{array}{ccc}\circ & \prime & \prime \prime \\
102 & 22 & 21.16 \\
143 & 06 & 43.08 \\
194 & 05 & 40.67 \\
279 & 36 & 09.33\end{array}$ & $\begin{array}{ccc}\circ & \prime & \prime \prime \\
282 & 19 & 37.28 \\
323 & 02 & 58.85 \\
14 & 06 & 46.96 \\
99 & 39 & 58.12\end{array}$ & \begin{tabular}{|c|} 
Log. meter8. \\
3.8446330 \\
4.1912142 \\
4.0539363 \\
3.9857795
\end{tabular} & $\begin{array}{r}\text { Miles. } \\
4.345 \\
9.651 \\
7.035 \\
6.014\end{array}$ \\
\hline
\end{tabular}

ROCKY BUTTE, SAN LUIS OBISPO COUNTY, CALIF.

A station of the U. S. Coast and Geodetic Survey.

On top of Rocky Butte in Santa Lucia Mountains, 14 miles from Cambria, 1.5 miles west of Newton Mather's house, 6 miles from house of J. P. Coffee. It may be reached by road along North Fork of San Simeon Creek. On east side of peak and 400 feet from summit is a good spring.

Station mark: A copper tack leaded in top of a stone 1 foot long and 8 inches square on top, 2 feet below surface, over which was built a concrete pier.

Reference marks: Four stones of same size as above with leaded bolts and copper tacks were placed, north, 47.12 inches; south, 44.38 inches; east, 31.25 inches; and west, 41.81 inches. The pier for observing latitude is 5.13 feet due west of station. Vertical circle wooden pier is N. $56.28 \mathrm{ft}$.

[Latitude $35^{\circ} 39^{\prime} 56.026^{\prime \prime}$. Longitude $121^{\circ} 03^{\prime} 32.063^{\prime \prime}$.]

\begin{tabular}{|c|c|c|c|c|}
\hline Tostation- & Azimuth. & Back azimuth. & Dista & nce. \\
\hline 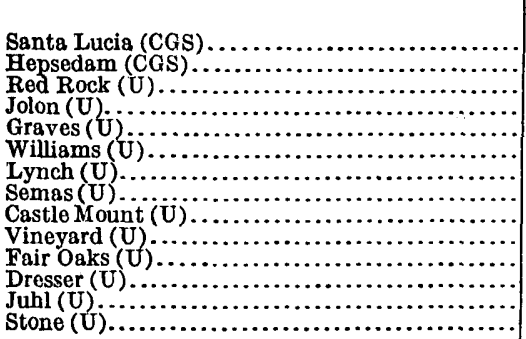 & $\begin{array}{ccc}\circ & \prime & \prime \prime \\
148 & 46 & 28.32 \\
196 & 18 & 58.11 \\
137 & 58 & 20.14 \\
160 & 33 & 09.81 \\
165 & 07 & 02.18 \\
189 & 20 & 56.31 \\
209 & 34 & 03.13 \\
220 & 44 & 28.41 \\
244 & 45 & 05.68 \\
249 & 01 & 10.69 \\
275 & 36 & 35.73 \\
281 & 43 & 00.18 \\
311 & 22 & 43.70 \\
333 & 26 & 24.10\end{array}$ & \begin{tabular}{rcc}
\multicolumn{1}{c}{} & $\prime$ & $\prime \prime$ \\
328 & 33 & 49.52 \\
16 & 27 & 15.09 \\
317 & 54 & 48.42 \\
340 & 28 & 15.18 \\
345 & 04 & 45.77 \\
9 & 22 & 58.39 \\
29 & 36 & 37.98 \\
40 & 52 & 43.59 \\
65 & 10 & 20.33 \\
69 & 14 & 41.10 \\
95 & 45 & 52.62 \\
102 & 00 & 00.26 \\
131 & 30 & 28.44 \\
153 & 30 & 12.07
\end{tabular} & \begin{tabular}{r|} 
Log. meters. \\
4.7951624 \\
4. 8756197 \\
4.1338783 \\
4.5783184 \\
4. 3578927 \\
4.5077331 \\
4.1301415 \\
4.5125744 \\
4.8558353 \\
4.5720691 \\
4.3829363 \\
4.6535228 \\
4.4285017 \\
4.3441912
\end{tabular} & $\begin{array}{r}\text { Miles. } \\
\text { 8. } 457 \\
23.533 \\
14.166 \\
20.002 \\
8.385 \\
20.227 \\
44.585 \\
23.196 \\
15.007 \\
27.982 \\
16.667 \\
13.726\end{array}$ \\
\hline
\end{tabular}

CGS $=$ U. S. Coast \& Geodetic Survey in 1884-85. U=C. F. Urguhart in 1917.

\section{RODGERS, MERCED COUNTY, CALIF.}

About 15 miles south of Merced and $1 \frac{1}{2}$ miles west and 0.5 mile south of El Nido School. It is on an east and west fence line and is 0.5 mile east of the house of $\mathrm{Mr}$. A. A. Rodgers.

Signal: Timber structure with cross targets and a flag.

Station mark: Iron bench mark centered under signal.

[Latitude $37^{\circ} 07^{\prime} 41.301^{\prime \prime}$. Longitude $120^{\circ} 31^{\prime} 20.071^{\prime \prime}$.]

\begin{tabular}{|c|c|c|c|c|}
\hline To station- & Azimuth. & Back azimuth. & Distar & \\
\hline 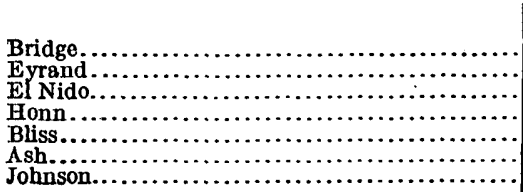 & $\begin{array}{rcc}\circ & \prime & \prime \prime \\
16 & 12 & 40.91 \\
165 & 56 & 52.64 \\
255 & 54 & 56.92 \\
256 & 08 & 53.25 \\
274 & 36 & 49.01 \\
293 & 32 & 03.76 \\
354 & 05 & 59.58\end{array}$ & \begin{tabular}{rcc}
\multicolumn{1}{c}{} & $\prime$ & $\prime \prime$ \\
196 & 11 & 43.79 \\
345 & 55 & 31.82 \\
75 & 56 & 05.06 \\
76 & 11 & 06.57 \\
94 & 41 & 02.16 \\
113 & 37 & 15.08 \\
174 & 06 & 17.46
\end{tabular} & \begin{tabular}{|r|} 
Log. meters. \\
3.9233892 \\
4.0564102 \\
3.4582780 \\
3.7897568 \\
4.0165461 \\
4.1431363 \\
3. 8528015
\end{tabular} & $\begin{array}{r}\text { Miles. } \\
5.209 \\
7.076 \\
1.785 \\
3.829 \\
6.455 \\
8.639 \\
4.427\end{array}$ \\
\hline
\end{tabular}


ROMERO, MERCED COUNTY, CALIF. (Not occupied.)

Cupola on the Romero School, about 5 miles northwest of town of Volta, on the Southern Pacific R. R.; road from Los Banos to Gustine passes school.

[Latitude $37^{\circ} 07^{\prime} 49.509^{\prime \prime}$. Longitude $121^{\circ} 00^{\prime} 54.540^{\prime \prime}$.]

\begin{tabular}{|c|c|c|c|c|}
\hline To station- & Azimuth. & Backazimuth. & Dista & ce. \\
\hline $\begin{array}{l}\text { Bareillas } \ldots \ldots \ldots \ldots \ldots \ldots \ldots \ldots \\
\text { Christian } \ldots \ldots \ldots \ldots \ldots \ldots \ldots \ldots \ldots \\
\text { Milepost } 162 \ldots \ldots \ldots \ldots \ldots \ldots \ldots\end{array}$ & \begin{tabular}{rcc}
$\circ$ & \multicolumn{1}{c}{} & $\prime \prime$ \\
10 & 15 & 53.96 \\
134 & 59 & 22.97 \\
269 & 27 & 52.77
\end{tabular} & $\begin{array}{rcc}\circ & \prime & \prime \prime \\
190 & 15 & 02.77 \\
314 & 56 & 31.66 \\
89 & 33 & 29.43\end{array}$ & \begin{tabular}{|r|} 
Log. meters. \\
4.0710566 \\
3.9951898 \\
4.1388420
\end{tabular} & $\begin{array}{r}\text { Miles. } \\
7.318 \\
6.145 \\
8.554\end{array}$ \\
\hline
\end{tabular}

ROUND, FRESNO COUNTY, CALIF.

On round hill north of Sanger.

Signal: A tree in cairn.

Station mark: Tablet in boulder.

[Latitude $36^{\circ} 49^{\prime} 08.926^{\prime \prime}$. Longitude $119^{\circ} 30^{\prime} 34.328^{\prime \prime}$.]

\begin{tabular}{|c|c|c|c|c|}
\hline Tostation- . & Azimuth. & Back azimuth. & Diste & \\
\hline 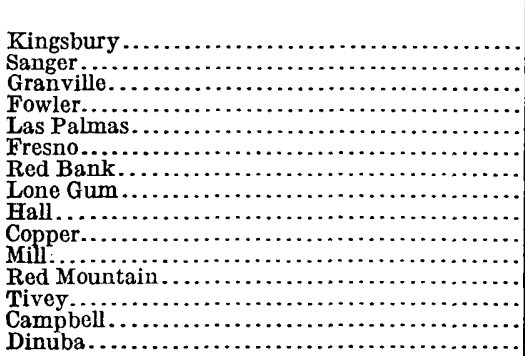 & \begin{tabular}{rcc}
\multicolumn{1}{c}{} & \multicolumn{1}{c}{$\prime$} \\
6 & 16 & 11.74 \\
18 & 48 & 49.59 \\
35 & 17 & 42.61 \\
35 & 13 & 37.15 \\
67 & 49 & 57.64 \\
69 & 56 & 52.01 \\
93 & 01 & 02.37 \\
110 & 18 & 14.03 \\
125 & 39 & 38.61 \\
136 & 08 & 41.18 \\
162 & 37 & 07.54 \\
243 & 33 & 22.06 \\
309 & 29 & 22.15 \\
329 & 58 & 04.92 \\
340 & 08 & 32.00
\end{tabular} & $\begin{array}{rcc}0 & \prime & \prime \prime \\
186 & 14 & 39.08 \\
198 & 47 & 08.97 \\
215 & 15 & 04.46 \\
215 & 07 & 40.97 \\
247 & 43 & 11.22 \\
249 & 46 & 51.75 \\
272 & 57 & 47.37 \\
290 & 13 & 37.66 \\
305 & 30 & 54.68 \\
316 & 03 & 47.42 \\
342 & 36 & 39.58 \\
63 & 37 & 20.49 \\
129 & 33 & 28.55 \\
150 & 01 & 11.96 \\
160 & 12 & 53.79\end{array}$ & $\begin{array}{r}\text { Log. meters. } \\
4.5309734 \\
4.1118698 \\
4.0547474 \\
4.4091957 \\
4.2595383 \\
4.4229203 \\
3.9071625 \\
4.0854889 \\
4.4245034 \\
4.2426860 \\
3.5881341 \\
4.0414461 \\
4.1215523 \\
4.1987067 \\
4.5123564\end{array}$ & $\begin{array}{r}\text { Miles. } \\
21.102 \\
8.039 \\
7.049 \\
15.942 \\
11.295 \\
16.454 \\
5.018 \\
-7.566 \\
16.514 \\
10.865 \\
2.407 \\
6.836 \\
8.221 \\
9.819 \\
20.217\end{array}$ \\
\hline
\end{tabular}

RUGG, FRESNO COUNTY, CALIF. (Not occupied.)

Station mark: Center of smokestack at pump station, at Rugg, on Southern Pacific R. R.

[Latitude $36^{\circ} 43^{\prime} 53.655^{\prime \prime}$. Longitude $120^{\circ} 00^{\prime} 15.307^{\prime \prime}$.]

\begin{tabular}{|c|c|c|c|c|}
\hline Tostation- & Azimuth. & Back azimuth. & Dista & ce. \\
\hline 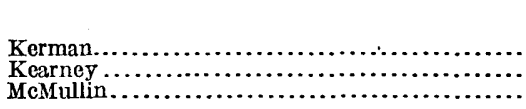 & $\begin{array}{ccc}\circ & \prime & \prime \prime \\
79 & 53 & 07.90 \\
277 & 33 & 46.27 \\
339 & 50 & 57.57\end{array}$ & \begin{tabular}{rcc}
\multicolumn{1}{c}{} & $\prime$ & $\prime \prime$ \\
259 & 51 & 07.07 \\
97 & 36 & 51.46 \\
159 & 52 & 09.67
\end{tabular} & \begin{tabular}{|r|} 
Log. meters. \\
3. 7069745 \\
3. 8894343 \\
3.9394826
\end{tabular} & $\begin{array}{r}\text { Miles. } \\
3.165 \\
4.817 \\
5.405\end{array}$ \\
\hline
\end{tabular}

SAMPSON, FRESHO COUNTY, CALIF.

On the point of a ridge, about 300 feet from the top, 4 miles northeast of Dunlap and one-eighth of a mile south of Gartners ranch house. It can be reached by taking the Millwood road from Dunlap $8 \frac{1}{2}$ miles to Sontag Point, thence going along the Sampson Flat road $3 \frac{1}{2}$ miles to the Gartner ranch house. A buckboard can be driven close to station. The station can also be reached by a trail from Dunlap.

Signal: Pine tree with white cloth, trimmed.40 feet from the ground. Position of signal computed.

Reference mark: Bronze tablet 40 feet east and 4 feet south of signal. 
[Latitude $36^{\circ} 46^{\prime} 09.745^{\prime \prime}$. Longitude $119^{\circ} 06^{\prime} 06.578^{\prime \prime}$.]

\begin{tabular}{|c|c|c|c|c|}
\hline Tostation- & Azimuth. & Back azimuth. & Dista & 20. \\
\hline 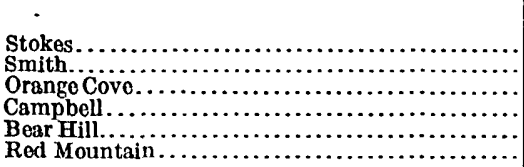 & \begin{tabular}{rcc}
\multicolumn{1}{c}{} & $\prime \prime$ & $\prime \prime$ \\
17 & 06 & 25.17 \\
46 & 25 & 13.77 \\
49 & 03 & 42.86 \\
74 & 07 & 27.20 \\
80 & 55 & 19.29 \\
111 & 31 & 53.52
\end{tabular} & $\begin{array}{ccc}\circ & \prime & \prime \prime \\
197 & 02 & 59.34 \\
226 & 16 & 30.30 \\
228 & 56 & 13.40 \\
253 & 55 & 59.90 \\
260 & 48 & 49.85 \\
291 & 21 & 12.27\end{array}$ & \begin{tabular}{|r} 
Log. meters. \\
4. 4649441 \\
4. 4782314 \\
4.3934012 \\
4.4721482 \\
4. 2134869 \\
4.4547651
\end{tabular} & $\begin{array}{r}\text { Miles. } \\
18.126 \\
18.689 \\
15.373 \\
18.429 \\
10.159 \\
17.706\end{array}$ \\
\hline
\end{tabular}

SANDY, FRESHO COUNTY, CALIF.

Station is on a sand dune 8 miles south and a little west of Kerman.

Signal: Quadripod with cross targets and flag.

Station mark: Iron bench mark centered under signal.

[Latitude $36^{\circ} 38^{\prime} 39.136^{\prime \prime}$. Longitude $120^{\circ} 07^{\prime} 28.232^{\prime \prime}$.]

\begin{tabular}{|c|c|c|c|c|}
\hline Tostation- & Azimuth. & Back azimuth. & Dista & ce. \\
\hline 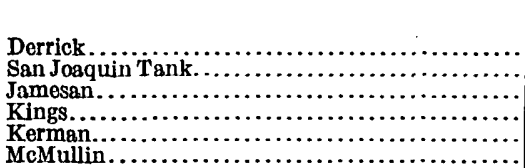 & \begin{tabular}{rcc}
\multicolumn{1}{c}{} & $\prime$ & $\prime \prime$ \\
18 & 36 & 34.98 \\
53 & 49 & 53.00 \\
144 & 22 & 09.42 \\
115 & 40 & 37.79 \\
213 & 03 & 39.50 \\
263 & 36 & 52.50
\end{tabular} & \begin{tabular}{rcc}
\multicolumn{1}{c}{} & $\prime$ & $\prime \prime$ \\
198 & 36 & 05.64 \\
233 & 47 & 36.11 \\
324 & 19 & 16.87 \\
295 & 36 & 57.51 \\
33 & 05 & 57.42 \\
83 & 42 & 22.96
\end{tabular} & \begin{tabular}{|l|} 
Log. meters. \\
3. 5830924 \\
3. 8490580 \\
4.0899247 \\
4.0070398 \\
4.0212703 \\
4. 1409783
\end{tabular} & $\begin{array}{r}\text { Miles. } \\
2.379 \\
4.389 \\
7.643 \\
6.315 \\
6.526 \\
8.597\end{array}$ \\
\hline
\end{tabular}

SANGER, FRESNO COUNTY, CALIF.

A large steel municipal watèr tank at Sanger.

Signal and station mark: Center of tank.

[Latitude $36^{\circ} 42^{\prime} 31.594^{\prime \prime}$. Longitude $119^{\circ} 33^{\prime} 22.436^{\prime \prime}$.]

\begin{tabular}{|c|c|c|c|c|}
\hline Tostation- & Azimuth. & Back azimuth. & Dista & \\
\hline 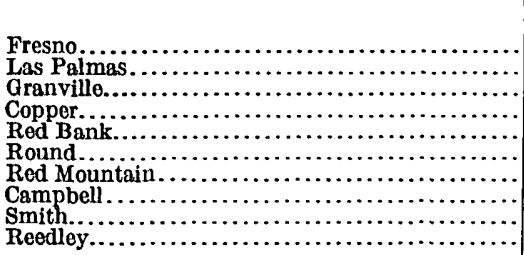 & \begin{tabular}{rcc}
\multicolumn{1}{c}{} & $\prime$ & $\prime \prime$ \\
98 & 40 & 13.87 \\
113 & 01 & 26.82 \\
141 & 25 & 04.02 \\
162 & 15 & 04.29 \\
162 & 53 & 58.19 \\
198 & 47 & 08.97 \\
219 & 14 & 59.77 \\
276 & 44 & 21.13 \\
306 & 35 & 20.16 \\
323 & 02 & 58.85
\end{tabular} & \begin{tabular}{rcc}
\multicolumn{1}{c}{$\circ$} & $\prime$ & $\prime \prime$ \\
278 & 31 & 54.90 \\
292 & 56 & 21.46 \\
321 & 24 & 06.59 \\
342 & 11 & 51.66 \\
342 & 52 & 24.06 \\
18 & 48 & 49.59 \\
39 & 20 & 38.57 \\
96 & 49 & 12.00 \\
126 & 42 & 53.45 \\
143 & 06 & 43.08
\end{tabular} & \begin{tabular}{|r|} 
Log. meters. \\
4.3210465 \\
4.1386317 \\
3.5822222 \\
4.4165379 \\
4.1224134 \\
4.1118698 \\
4.3453730 \\
4.0851381 \\
4.3713314 \\
4.1912142
\end{tabular} & $\begin{array}{r}\text { Miles. } \\
13.014 \\
8.550 \\
2.375 \\
16.214 \\
8.237 \\
8.039 \\
13.763 \\
7.559 \\
14.611 \\
9.651\end{array}$ \\
\hline
\end{tabular}

SAN JOAQUIN TANK, FRESNO COUNTY, CALIF. (Not Occupied,)

Station mark: Center of white tank house (municipal) in town of San Joaquin.

[Latitude $36^{\circ} 36^{\prime} 23.826^{\prime \prime}$. Longitude $120^{\circ} 11^{\prime} 17.696^{\prime \prime}$.]

\begin{tabular}{|c|c|c|c|c|}
\hline Tostation-- & Azimuth. & Back azimuth. & Dista & \\
\hline 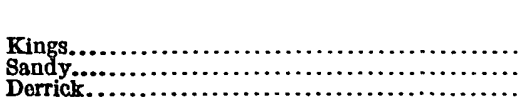 & $\begin{array}{ccc}\circ & , & \prime \prime \\
158 & 00 & 08.29 \\
233 & 47 & 36.11 \\
263 & 05 & 18.87\end{array}$ & \begin{tabular}{rcc}
\multicolumn{1}{c}{0} & $\prime$ & $\prime \prime$ \\
337 & 58 & 45.06 \\
53 & 49 & 53.00 \\
83 & 07 & 06.39
\end{tabular} & \begin{tabular}{|r|} 
Log. meters. \\
3. 9658451 \\
3. 8490580 \\
3.6544898
\end{tabular} & $\begin{array}{r}\text { Miles. } \\
5.744 \\
4.389 \\
2.804\end{array}$ \\
\hline
\end{tabular}

$121.166^{\circ}-23-B u l l .709-37$ 


\section{SAN JUAN, MERCED COUNTY, CALIF.}

The center of a water tank on the Miller \& Lux San Juan ranch, 8 miles north of Dos Palos.

Signal: Center of tank.

Station mark: Center of concrete foundation of tank.

[Latitude $37^{\circ} 04^{\prime} 50.812^{\prime \prime}$. Longitude $120^{\circ} 39^{\prime} 23.921^{\prime \prime}$.]

\begin{tabular}{|c|c|c|c|c|}
\hline Tostation- & Azimuth. & Back azimuth. & Dista & 20. \\
\hline 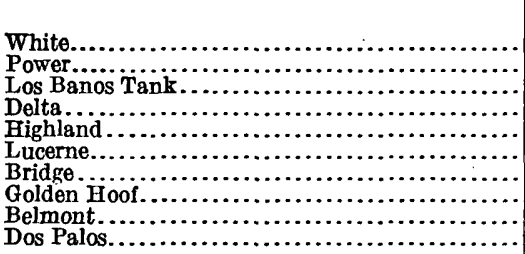 & $\begin{array}{rcc}\circ & \prime & \prime \prime \\
41 & 30 & 42.69 \\
62 & 55 & 24.86 \\
82 & 33 & 57.46 \\
119 & 02 & 56.36 \\
133 & 25 & 01.88 \\
262 & 35 & 27.97 \\
286 & 10 & 56.47 \\
315 & 15 & 11.46 \\
328 & 18 & 35.13 \\
344 & 56 & 25.16\end{array}$ & \begin{tabular}{rcc}
\multicolumn{1}{c}{} & $\prime$ & $\prime \prime$ \\
221 & 24 & 03.49 \\
242 & 53 & 10.08 \\
262 & 27 & 08.23 \\
298 & 59 & 57.09 \\
313 & 21 & 03.91 \\
82 & 37 & 44.66 \\
106 & 14 & 51.03 \\
135 & 20 & 48.85 \\
148 & 19 & 47.67 \\
164 & 57 & 33.16
\end{tabular} & \begin{tabular}{r|} 
Log. meters. \\
4.3940425 \\
3.7927464 \\
4.2282697 \\
3.9239558 \\
4.1268982 \\
3.7517153 \\
4.0005040 \\
4.2943323 \\
3.7529990 \\
4.0312537
\end{tabular} & $\begin{array}{r}\text { Miles. } \\
15.396 \\
3.856 \\
10.510 \\
5.216 \\
8.322 \\
3.508 \\
6.221 \\
12.237 \\
3.518 \\
6.677\end{array}$ \\
\hline
\end{tabular}

SANTA LUCIA, MONTEREY COUNTY, CALIF.

A station of the United States Coast and Geodetic Survey on eastern summit of highest peak of Santa Lucia Range. Best reached by wagon road up San Antonio Valley from Jolon to a point 13 miles past old mission; here leave road, turn to right up flat ridge for 1 mile, cross canyon to east and keep up ridge for 1 mile to just past an old $\log$ cabin; recross canyon to left side and follow trail to summit, rising 3,500 feet in about 4 miles.

Station mark: Cross in end of copper bolt in solid rock, over which is a concrete foundation 3 feet square and 10 inches deep, on which is a concrete pier 26 inches in diameter with a copper bolt with cross marking station center.

Reference marks: Holes drilled in outcropping rocks filled with lead, into which copper tacks were driven, and distant as follows: N. 6.88 feet, S. 7.18 feet, E. 5.91 feet, W. 10.08 feet. Concrete pier occupied for latitude in 1885 was 44 feet 1 inch from station in azimuth $227^{\circ} 28^{\prime}$. The azimuth from center of station to frame pier used for the vertical circle in 1885 was $56^{\circ} 14^{\prime}$ and distance was 5.486 meters. Stations occupied by the eclipse expedition of 1880 were a little north of west from the station and distant from it 546 feet and over.

[Latitude $36^{\circ} 08^{\prime} 45.328^{\prime \prime}$. Longitude $121^{\circ} 25^{\prime} 05.937^{\prime \prime}$.]

\begin{tabular}{|c|c|c|c|c|}
\hline Tostation- & Azimuth. & Back azimuth. & Dista & nce. \\
\hline 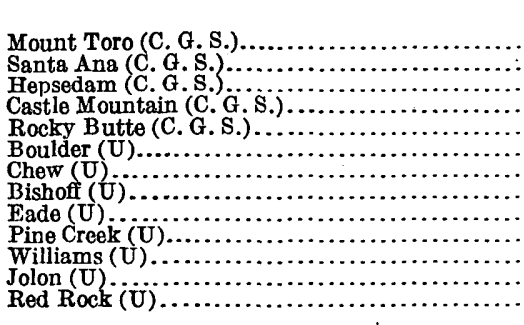 & $\begin{array}{ccc}\circ & \prime & \prime \prime \\
157 & 58 & 56.29 \\
191 & 06 & 13.77 \\
250 & 29 & 21.13 \\
282 & 57 & 22.08 \\
328 & 33 & 49.52 \\
128 & 53 & 00.84 \\
143 & 50 & 46.95 \\
255 & 50 & 46.80 \\
257 & 32 & 40.11 \\
285 & 20 & 29.35 \\
299 & 40 & 26.76 \\
311 & 35 & 13.13 \\
331 & 32 & 31.97\end{array}$ & \begin{tabular}{rcc}
\multicolumn{1}{c}{} & $\prime$ & $\prime \prime$ \\
337 & 52 & 09.62 \\
11 & 12 & 51.48 \\
70 & 50 & 25.69 \\
103 & 35 & 26.85 \\
148 & 46 & 28.32 \\
308 & 39 & 54.41 \\
323 & 45 & 29.24 \\
75 & 58 & 30.31 \\
77 & 48 & 34.75 \\
105 & 27 & 13.69 \\
119 & 55 & 10.96 \\
131 & 42 & 58.53 \\
151 & 41 & 38.66
\end{tabular} & \begin{tabular}{|r} 
Log. meters. \\
4.6584603 \\
4.9339816 \\
4.7530109 \\
4.9994343 \\
4.7951624 \\
4.6290707 \\
4.3566443 \\
4.3061705 \\
4.6165346 \\
4.2501683 \\
4.6368799 \\
4.4228507 \\
4.6910257
\end{tabular} & 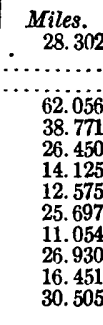 \\
\hline
\end{tabular}

C. G. S.=U. S. Coast and Geodetic Survey in 1885. U=C. F. Urquhart in 1917. 
SHELI NO. 1, FRESNO COUNTY, CAIIF. (Not occupied.)

Station is large smokestack at the Shell oil station about 10 miles north-northwest of Mendota, a small town on the Southern Pacific R. R.

[Latitude $36^{\circ} 46^{\prime} 26.216^{\prime \prime}$. Longitude $120^{\circ} 41^{\prime} 09.250^{\prime \prime}$.]

\begin{tabular}{|c|c|c|c|c|}
\hline Tostation- & Azimuth. & Backazimuth. & Dista & ce. \\
\hline 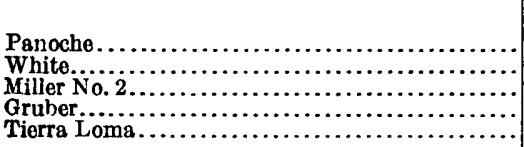 & $\begin{array}{rcc}\circ & \prime & \prime \prime \\
52 & 33 & 44.04 \\
138 & 16 & 40.08 \\
205 & 15 & 50.50 \\
283 & 41 & 06.99 \\
333 & 32 & 32.07\end{array}$ & \begin{tabular}{rcc}
\multicolumn{1}{c}{} & \multicolumn{1}{c}{ ' } & $\prime \prime$ \\
232 & 30 & 56.09 \\
318 & 11 & 05.45 \\
25 & 17 & 16.80 \\
103 & 43 & 06.23 \\
153 & 33 & 33.21
\end{tabular} & \begin{tabular}{r|} 
Iog. meters. \\
3.9431269 \\
4.3171585 \\
3.9223302 \\
3.9062818 \\
3.7552258
\end{tabular} & $\begin{array}{r}\text { Miles. } \\
5.451 \\
12.898 \\
5.196 \\
3.160 \\
3.537\end{array}$ \\
\hline
\end{tabular}

SHELL NO. 2, FRESNO COUNTY, CALIF. (Not occupied.)

Center of stack at the Shell oil station, about 15 miles west of Tranquility, on the Southern Pacific R. R.

[Latitude $36^{\circ} 35^{\prime} 25.506^{\prime \prime}$. Longitude $120^{\circ} 31^{\prime} 07.939^{\prime \prime}$.]

\begin{tabular}{|c|c|c|c|c|}
\hline Tostation- & Azimuth. & Backanimuth. & Distar & ce. \\
\hline 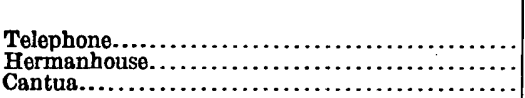 & $\begin{array}{ccc}\circ & \prime & \prime \prime \\
182 & 00 & 53.54 \\
226 & 28 & 37.35 \\
303 & 58 & 57.54\end{array}$ & $\begin{array}{rcc}\circ & \prime & \prime \prime \\
2 & 01 & 03.04 \\
46 & 35 & 53.96 \\
124 & 04 & 05.82\end{array}$ & $\begin{array}{r}\text { Log. meters. } \\
4.0508452 \\
4.3981829 \\
4.1912724\end{array}$ & $\begin{array}{r}\text { Miles. } \\
6.985 \\
15.543 \\
9.652\end{array}$ \\
\hline
\end{tabular}

SCHOOL, MERCED COUNTY, CALIF. (Not occupled.)

Cupola on schoolhouse about 3 miles southwest of South End of Barn, at Joe Silva's ranch, 9 miles east of the town of Newman, on Southern Pacific R. R.

[Latitude $37^{\circ} 19^{\prime} 29.650^{\prime \prime}$. Longitude $120^{\circ} 54^{\prime} 31.740^{\prime \prime}$.]

\begin{tabular}{|c|c|c|c|c|}
\hline Tostation- & Azimuth. & Back azimuth. & \multicolumn{2}{|c|}{ Distance. } \\
\hline $\begin{array}{l}\text { South End of Barn } \ldots \ldots \ldots \ldots \ldots \ldots \ldots \ldots \ldots \ldots \ldots \ldots \ldots \ldots \ldots \\
\text { Crow } . . . \ldots \ldots \ldots \ldots \ldots\end{array}$ & \begin{tabular}{rcc}
\multicolumn{1}{c}{$c$} & $\prime \prime$ & $\prime \prime$ \\
233 & 23 & 35.98 \\
99 & 42 & 01.03
\end{tabular} & $\begin{array}{ccc}\circ & \prime & \prime \prime \\
53 & 24 & 58.85 \\
279 & 33 & 20.85\end{array}$ & \begin{tabular}{|r|} 
Log. meters. \\
3.6221862 \\
4.3306488
\end{tabular} & $\begin{array}{r}\text { Miles. } \\
2.603 \\
13.305\end{array}$ \\
\hline
\end{tabular}

SEMAS, MONTEREY COUNTY, CALIF.

On a very prominent hill 2.5 or 3 miles north from Bradley and on the east side of Salinas River and the Southern Pacific R. R. The land has been owned by a man named Semas, but the name is probably not well known. Animals can be ridden to summit by entering pasture at Bradley and going north down Salinas River to the first canyon, thence up canyon 0.5 mile to a small level place; cross canyon; thence go north up gulch through a gate and uphill to station.

Signal: An oak tree set in ground on most northern peak.

Station mark: A triangulation tablet set in rock placed in ground 1 meter north of signal. Position of tablet was computed. Azimuth from tablet to signal, $355^{\circ} 45^{\prime}$; distance, 1.00 meter.

[Latitude $35^{\circ} 53^{\prime} 15.437^{\prime \prime}$. Longitude $120^{\circ} 49^{\prime} 25.039^{\prime \prime}$.]

\begin{tabular}{|c|c|c|c|c|}
\hline Tostation- & Azimuth. & Back azimuth. & Dista & ce. \\
\hline 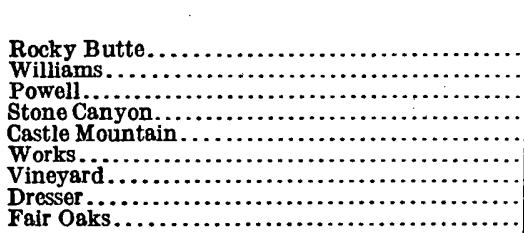 & $\begin{array}{rcc}\circ & \prime & \prime \prime \\
40 & 52 & 43.59 \\
114 & 02 & 47.66 \\
217 & 04 & 50.29 \\
237 & 54 & 46.28 \\
262 & 23 & 07.28 \\
294 & 39 & 10.40 \\
309 & 49 & 44.67 \\
326 & 05 & 03.50 \\
354 & 14 & 29.55\end{array}$ & \begin{tabular}{rcc}
\multicolumn{1}{c}{} & $\prime$ & $\prime \prime$ \\
220 & 44 & 28.41 \\
293 & 56 & 33.17 \\
37 & 08 & 35.71 \\
58 & 04 & 18.15 \\
82 & 40 & 09.16 \\
114 & 47 & 13.46 \\
129 & 55 & 01.36 \\
146 & 13 & 51.66 \\
174 & 15 & 32.89
\end{tabular} & $\begin{array}{r}\text { Log. meters. } \\
4.5125744 \\
4.2434984 \\
4.2028012 \\
4.4591241 \\
4.6439980 \\
4.3577320 \\
4.2476951 \\
4.6107150 \\
4.4340556\end{array}$ & $\begin{array}{r}\text { Miles. } \\
20.227 \\
10.886 \\
9.912 \\
17.884 \\
27.375 \\
14.161 \\
10.995 \\
25.355 \\
16.881\end{array}$ \\
\hline
\end{tabular}


SERVICE, MADERA COUNTY, CAIIF.

Station is windmill at the Service School, about 8 miles east of Madera.

Signal: Center of mill.

Station mark: Eight-inch well under mill.

[Latitude $36^{\circ} 58^{\prime} 01.977^{\prime \prime}$. Longitude $119^{\circ} 55^{\prime} 15.767^{\prime \prime}$.]

\begin{tabular}{|c|c|c|c|c|}
\hline Tostation- & Azimuth. & Back azimuth. & Dista & \\
\hline 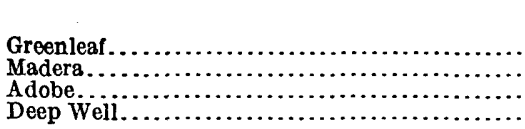 & $\begin{array}{rcc}\circ & \prime & \prime \prime \\
7 & 58 & 11.29 \\
86 & 25 & 03.20 \\
215 & 30 & 32.59 \\
311 & 38 & 01.94\end{array}$ & \begin{tabular}{rcc}
\multicolumn{1}{c}{} & $\prime$ & $\prime \prime$ \\
187 & 57 & 38.84 \\
266 & 20 & 00.20 \\
35 & 32 & 41.76 \\
131 & 40 & 30.58
\end{tabular} & $\begin{array}{r}\text { Log. meters. } \\
3.9844702 \\
4.0965713 \\
3.9606108 \\
3.9133288\end{array}$ & $\begin{array}{r}\text { Miles. } \\
5.995 \\
7.761 \\
5.675 \\
5.090\end{array}$ \\
\hline
\end{tabular}

SHARON, MADERA COUNTY, CALIF.

A windmill at Sharon, a station on the Atchison, Topeka \& Santa Fe Ry. between Merced and Fresno. The mill is at the north end of a warehouse on the east side of the track, and is opposite the station.

Signal: Center of mill.

Station mark: Pipe in the ground.

[Latitude $37^{\circ} 06^{\prime} 01.238^{\prime \prime}$. Longitude $120^{\circ} 07^{\prime} 50.951^{\prime \prime}$.]

\begin{tabular}{|c|c|c|c|c|}
\hline Tostation- & Azimuth. & Back azimuth. & Dista & ce. \\
\hline 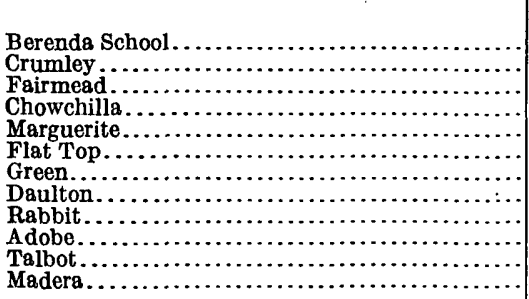 & $\begin{array}{rcc}\bullet & \prime & \prime \prime \\
15 & 43 & 40.65 \\
19 & 12 & 51.71 \\
64 & 55 & 26.89 \\
103 & 58 & 35.69 \\
150 & 27 & 10.57 \\
185 & 27 & 36.12 \\
214 & 09 & 25.33 \\
254 & 55 & 57.30 \\
257 & 07 & 28.40 \\
286 & 56 & 46.08 \\
308 & 35 & 11.51 \\
338 & 12 & 10.96\end{array}$ & \begin{tabular}{rcc}
\multicolumn{1}{c}{} & $\prime$ & $\prime \prime$ \\
195 & 42 & 53.00 \\
199 & 11 & 33.52 \\
244 & 53 & 14.16 \\
283 & 53 & 53.22 \\
330 & 25 & 36.57 \\
5 & 28 & 20.74 \\
34 & 14 & 30.93 \\
75 & 01 & 18.90 \\
77 & 14 & 43.27 \\
107 & 06 & 30.82 \\
128 & 38 & 54.10 \\
158 & 14 & 42.44
\end{tabular} & \begin{tabular}{|r|} 
Log. meters. \\
3.8577427 \\
3.9888415 \\
3.7783343 \\
4.0758752 \\
3.8916362 \\
4.2812386 \\
4.3461516 \\
4.1342778 \\
4.2611284 \\
4.3990592 \\
4.0672925 \\
4.2242262
\end{tabular} & $\begin{array}{r}\text { Miles. } \\
4.478 \\
6.056 \\
3.730 \\
7.400 \\
4.842 \\
11.874 \\
13.788 \\
8.465 \\
11.336 \\
15.574 \\
7.255 \\
10.413\end{array}$ \\
\hline
\end{tabular}

SILAXO, FRESNO COUNTY, CALIF.

In a pasture field owned by Miller \& Lux, Inc., 0.5 mile west of milepost 136 on the Pipe Line road, 4 miles northwest of Firebaugh and about 0.6 mile south of where same road crosses canal. It is 1 mile south and a little west of Silaxo oil pumping station.

Signal: Timber quadripod with cross targets and flag.

Station mark: Iron bench-mark post centered under signal.

[Latitude $36^{\circ} 52^{\prime} 40.970^{\prime \prime}$. Longitude $120^{\circ} 31^{\prime} 46.377^{\prime \prime}$.]

\begin{tabular}{|c|c|c|c|c|}
\hline To station- & Azimuth. & Back azimuth. & Distar & \\
\hline 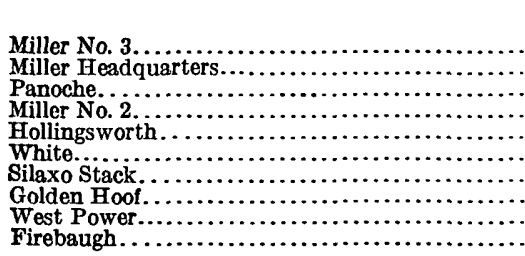 & $\begin{array}{rcc}0 & \prime & \prime \prime \\
11 & 23 & 01.13 \\
17 & 50 & 15.45 \\
51 & 08 & 52.04 \\
69 & 00 & 00.82 \\
91 & 45 & 08.58 \\
98 & 09 & 05.30 \\
191 & 00 & 24.55 \\
196 & 39 & 42.84 \\
227 & 27 & 28.48 \\
287 & 13 & 06.31\end{array}$ & \begin{tabular}{rcc}
\multicolumn{1}{c}{} & $\prime$ & $\prime \prime$ \\
191 & 21 & 55.80 \\
197 & 49 & 17.60 \\
231 & 00 & 26.69 \\
248 & 55 & 49.57 \\
271 & 41 & 21.47 \\
277 & 57 & 52.34 \\
11 & 00 & 36.23 \\
16 & 40 & 44.61 \\
47 & 29 & 54.29 \\
107 & 15 & 42.11
\end{tabular} & \begin{tabular}{|r} 
Log. meters. \\
4. 1367072 \\
3.8924525 \\
4.4294245 \\
4.0459480 \\
3. 9720590 \\
4.4476319 \\
3.4020890 \\
3.9480602 \\
3.9114933 \\
3.8282818
\end{tabular} & $\begin{array}{r}\text { Miles. } \\
8.513 \\
4.851 \\
16.702 \\
6.907 \\
5.827 \\
17.417 \\
1.568 \\
5.513 \\
5.068 \\
4.184\end{array}$ \\
\hline
\end{tabular}


SILAXO STACK, FRESNO COUNTY, CAIIF, (Not occupled.)

Station is the smokestack at oil station at Silaxo, on Southern Pacific Railroad between Firebaugh and South Dos Palos.

[Latitude $36^{\circ} 54^{\prime} 01.342^{\prime \prime}$. Longitude $120^{\circ} 31^{\prime} 26.912^{\prime \prime}$.]

\begin{tabular}{|c|c|c|c|c|}
\hline Tostation- & Azimuth. & Back azimuth. & Dista & \\
\hline 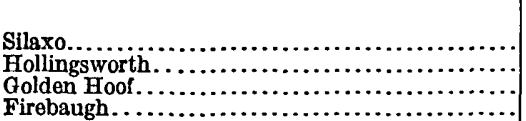 & \begin{tabular}{rcc}
$\circ$ & \multicolumn{1}{c}{,$\prime$} \\
11 & 00 & 36.23 \\
77 & 28 & 11.58 \\
198 & 54 & 14.51 \\
306 & 55 & 23.90
\end{tabular} & $\begin{array}{rcc}\circ & \prime & \prime \prime \\
191 & 00 & 24.55 \\
257 & 24 & 12.73 \\
18 & 55 & 04.61 \\
126 & 57 & 48.06\end{array}$ & $\begin{array}{c}\text { Log. meters. } \\
\text { 3. } 4020890 \\
4.0041040 \\
3.8038730 \\
3.8717451\end{array}$ & $\begin{array}{r}\text { Miles. } \\
1.568 \\
6.273 \\
3.956 \\
4.625\end{array}$ \\
\hline
\end{tabular}

SMITH, FRESNO COUNTY, CAITF.

The highest point of Smith Mountain, about 6 miles southeast of Reedley.

Signal: Cairn 8 feet high.

Station mark: Chiseled circle under center of cairn.

Reference mark: Tablet 3.2 feet south of center of cairn. Position of signal computed.

[Latitude $36^{\circ} 34^{\prime} 56.212^{\prime \prime}$. Longitude $119^{\circ} 20^{\prime} 42.990^{\prime \prime}$.]

\begin{tabular}{|c|c|c|c|c|}
\hline To station- & Azimuth. & Back azimuth. & Distar & \\
\hline 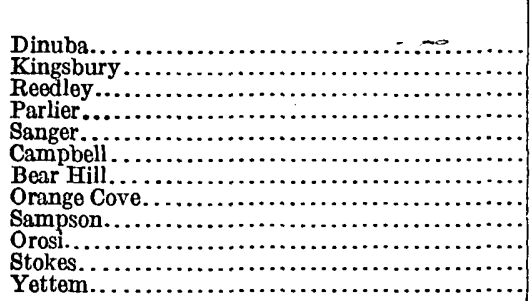 & $\begin{array}{rcc}\circ & \prime & \prime \prime \\
40 & 13 & 38.20 \\
67 & 58 & 15.35 \\
99 & 39 & 58.12 \\
100 & 49 & 40.73 \\
126 & 42 & 53.45 \\
151 & 45 & 13.20 \\
197 & 07 & 29.62 \\
214 & 16 & 21.22 \\
226 & 16 & 30.30 \\
295 & 36 & 57.87 \\
293 & 16 & 16.63 \\
321 & 13 & 06.53\end{array}$ & \begin{tabular}{rcc}
\multicolumn{1}{c}{} & $\prime$ & $\prime \prime$ \\
220 & 12 & 10.84 \\
247 & 50 & 54.45 \\
279 & 36 & 09.33 \\
280 & 43 & 08.05 \\
306 & 35 & 20.16 \\
331 & 42 & 30.42 \\
17 & 09 & 44.36 \\
34 & 17 & 35.31 \\
46 & 25 & 13.77 \\
115 & 40 & 01.30 \\
118 & 21 & 33.18 \\
141 & 16 & 31.00
\end{tabular} & \begin{tabular}{|c|} 
Log. meters. \\
3. 7529353 \\
4. 2982711 \\
3. 9857795 \\
4. 2218216 \\
4. 3713314 \\
4. 1555507 \\
4. 2790135 \\
3. 7389986 \\
4.4782314 \\
3. 9290562 \\
4.1765766 \\
4.1351443
\end{tabular} & $\begin{array}{r}\text { Miles. } \\
3.518 \\
12.349 \\
6.014 \\
10.356 \\
14.611 \\
8.890 \\
11.813 \\
3.407 \\
18.689 \\
5.277 \\
9.331 \\
8.482\end{array}$ \\
\hline
\end{tabular}

SOUTHEAST COTTONWOOD, MADERA COUNTY, CALIF., FROM NORTH.

Station is the more southeastern of two cottonwood trees in pasture about 10 miles west of Madera and north of the Madera and Firebaugh road. It can be reached by auto.

Signal and station mark: Cottonwood tree. Position of signal computed.

Reference mark: A U. S. Geological Survey bench-mark post. Azimuth from tablet to signal, $92^{\circ} 20^{\prime}$; distance, 16.5 feet.

[Latitude $36^{\circ} 56^{\prime} 20.240^{\prime \prime}$ (from north), $36^{\circ} 56^{\prime} 20.244^{\prime \prime}$ (from south), $36^{\circ} 56^{\prime} 20.360^{\prime \prime}$ (from west). Longitude $120^{\circ} 15^{\prime} 08.409^{\prime \prime}$ (from north), $120^{\circ} 15^{\prime} 08.554^{\prime \prime}$ (from south), $120^{\circ} 15^{\prime} 08.613^{\prime \prime}$ (from west).]

\begin{tabular}{|c|c|c|c|c|}
\hline To station- & Aximuth. & Back azimuth. & Dista & \\
\hline 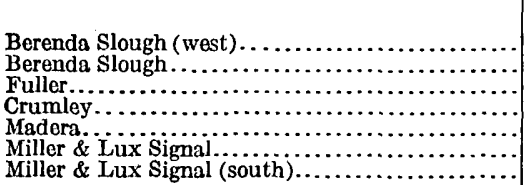 & $\begin{array}{lcc}\circ & , & \prime \prime \\
143 & 17 & 21.80 \\
143 & 17 & 30.32 \\
188 & 04 & 14.33 \\
221 & 07 & 57.90 \\
262 & 06 & 02.76 \\
274 & 01 & 41.48 \\
274 & 01 & 50.24\end{array}$ & \begin{tabular}{rcc}
\multicolumn{1}{c}{} & $\prime$ & $\prime \prime$ \\
323 & 14 & 44.48 \\
323 & 14 & 31.48 \\
8 & 0.4 & 38.18 \\
41 & 11 & 02.99 \\
82 & 12 & 56.92 \\
91 & 04 & 40.04 \\
94 & 04 & 48.78
\end{tabular} & $\begin{array}{r}\text { Loo. metcrs. } \\
\text { 4. } 0 \pm 22536 \\
\text { 4. } 0122536 \\
3.8442016 \\
\text { 4. } 0630925 \\
4.2357339 \\
3.8675666 \\
\text { 3. } 8675076\end{array}$ & $\begin{array}{r}\text { Mrlles. } \\
6.849 \\
6.849 \\
4.341 \\
7.185 \\
10.693 \\
4.581 \\
4.580\end{array}$ \\
\hline
\end{tabular}


SOUTHEAST COTTONWOOD, MADERA COUNTY, CALIF., FROM SOUTH.

[Latitude $36^{\circ} 56^{\prime} 20.244^{\prime \prime}$ (from south), $36^{\circ} 56^{\prime} 20.240^{\prime \prime}$ (from north), $36^{\circ} 56^{\prime} 20.360^{\prime \prime}$ (from west.). Longitude $120^{\circ} 15^{\prime} 08.554^{\prime \prime}$ (from south), $120^{\circ} 15^{\prime} 08.409^{\prime \prime}$ (from north), $120^{\circ} 15^{\prime} 08.613^{\prime \prime}$ (from west).

\begin{tabular}{|c|c|c|c|c|}
\hline Tostation- & Azimuth. & Back azimuth. & Distar & se. \\
\hline 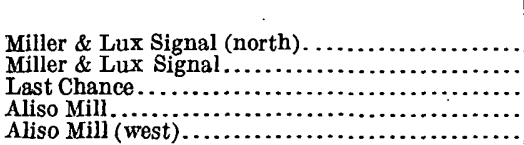 & \begin{tabular}{rcc}
\multicolumn{1}{c}{} &, & $\prime \prime$ \\
274 & 01 & 41.04 \\
274 & 01 & 50.24 \\
314 & 27 & 47.96 \\
2 & 05 & 04.62 \\
2 & 04 & 46.63
\end{tabular} & \begin{tabular}{rcc}
\multicolumn{1}{c}{} &, & $\prime \prime$ \\
94 & 04 & 40.04 \\
94 & 04 & 48.78 \\
134 & 31 & 19.05 \\
182 & 04 & 57.06 \\
182 & 04 & 39.08
\end{tabular} & $\begin{array}{r}\text { Log. meters. } \\
\text { 3. } 8675666 \\
3.8675076 \\
4.0864039 \\
3.9335728 \\
3.9336920\end{array}$ & $\begin{array}{r}\text { Miles. } \\
4.581 \\
4.580 \\
7.581 \\
5.332 \\
5.334\end{array}$ \\
\hline
\end{tabular}

SOUTHEAST COTTONWOOD, MADERA COUNTY, CALIF., FROM WEST.

[Latitude $36^{\circ} 56^{\prime} 20.360^{\prime \prime}$ (from west), $36^{\circ} 56^{\prime} 20.240^{\prime \prime}$ (from north), $36^{\circ} 56^{\prime} 20.244^{\prime \prime}$ (from south). Longitude $120^{\circ} 15^{\prime} 08.613^{\prime \prime}$ (from west), $120^{\circ} 15^{\prime} 08.409^{\prime \prime}$ (from north), $120^{\circ} 15^{\prime} 08.554^{\prime \prime}$ (from south).]

\begin{tabular}{|c|c|c|c|c|}
\hline To station- & Azimuth. & Back azimuth. & Dista1 & ce. \\
\hline 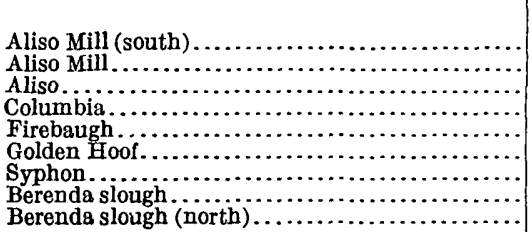 & $\begin{array}{rcc}\circ & \prime & \prime \prime \\
2 & 05 & 04.62 \\
2 & 04 & 46.63 \\
37 & 48 & 42.82 \\
42 & 52 & 38.91 \\
64 & 27 & 11.90 \\
94 & 33 & 35.42 \\
120 & 38 & 14.28 \\
143 & 17 & 24.80 \\
143 & 17 & 30.32\end{array}$ & $\begin{array}{ccc}\circ & \prime & \prime \prime \\
182 & 04 & 57.06 \\
182 & 04 & 39.08 \\
217 & 46 & 26.93 \\
222 & 48 & 46.00 \\
244 & 19 & 48.74 \\
274 & 24 & 37.50 \\
300 & 33 & 54.45 \\
323 & 14 & 44.48 \\
323 & 14 & 31.48\end{array}$ & \begin{tabular}{|r|} 
Log. meters. \\
3.9335728 \\
3.9336920 \\
3.9610265 \\
4.1424330 \\
4.3067411 \\
4.3465805 \\
4.0940288 \\
4.0422536 \\
4.0422536
\end{tabular} & $\begin{array}{r}\text { Miles. } \\
5.332 \\
5.334 \\
5.680 \\
8.625 \\
12.592 \\
13.802 \\
7.716 \\
6.849 \\
6.849\end{array}$ \\
\hline
\end{tabular}

SOUTH END BARN, MERCED COUNTY, CALIF.

A large white barn on the Joseph Silva ranch, 9 miles east of Newman, on the Newman-Livingston road.

Signal and station mark: South end of barn.

[Latitude $37^{\circ} 20^{\prime} 50.672^{\prime \prime}$. Longitude $120^{\circ} 52^{\prime} 15.093^{\prime \prime}$.]

\begin{tabular}{|c|c|c|c|c|}
\hline Tostation- & Azimuth. & Back azimuth. & Distan & se. \\
\hline 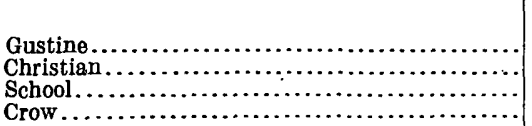 & $\begin{array}{lcc}\stackrel{\circ}{1} & \prime & \prime \prime \\
48 & 13 & 19.20 \\
49 & 14 & 42.83 \\
53 & 24 & 58.85 \\
92 & 37 & 07.81\end{array}$ & $\begin{array}{ccc}\circ & \prime & \prime \prime \\
228 & 08 & 37.94 \\
229 & 06 & 36.52 \\
233 & 23 & 35.98 \\
272 & 27 & 04.59\end{array}$ & \begin{tabular}{|r|} 
Log. meter 8. \\
4.1906813 \\
4.4174049 \\
3.6221862 \\
4.3890577
\end{tabular} & $\begin{array}{r}\text { Miles. } \\
9.639 \\
16.246 \\
2.603 \\
15.220\end{array}$ \\
\hline
\end{tabular}

STOCKDALE, MONTEREY COUNTY, CALIF.

On highest point of a low brushy mountain, about 12 miles from Parkfield and 5 miles from Stone Canyon, on west side of road between those two places. It is west of and about 0.75 mile from Mr. Stockdale's camp and new ranch. Mr. Stockdale's place is less than 1 mile from the county road. Animals can be ridden to the summit of hill.

Signal: A pine tree set in the ground on highest part of the hill.

Station mark: A triangulation tablet. Position of table computed. Azimuth from tablet to signal, $134^{\circ} 46^{\prime}$; distance, 2.00 meters.

[Latitude $35^{\circ} 59^{\prime} 15.344^{\prime \prime}$. Longitude $120^{\circ} 34^{\prime} 51.150^{\prime \prime}$.]

\begin{tabular}{|c|c|c|c|c|}
\hline Tostation- & Azimuth. & Back azimuth. & Dista & \\
\hline 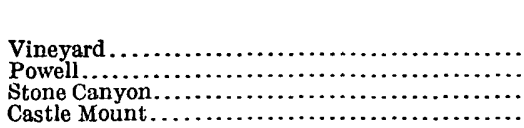 & \begin{tabular}{rcc}
\multicolumn{1}{c}{$\cdot$} & $\prime$ & $\prime \prime$ \\
20 & 26 & 52.04 \\
97 & 35 & 52.05 \\
211 & 02 & 03.78 \\
283 & 46 & 36.87
\end{tabular} & \begin{tabular}{rcc}
\multicolumn{1}{c}{} & $\prime$ & $\prime \prime$ \\
200 & 23 & 36.85 \\
277 & 31 & 04.14 \\
31 & 03 & 02.59 \\
103 & 55 & 06.76
\end{tabular} & $\begin{array}{r}\text { Log. meters. } \\
4.3790687 \\
4.0926416 \\
3.6865267 \\
4.3503908\end{array}$ & $\begin{array}{r}\text { Miles. } \\
14.874 \\
7.691 \\
3.019 \\
13.923\end{array}$ \\
\hline
\end{tabular}


STOKES, TULARE COUNTY, CALIF.

On a sharp butte in the middle of Stokes Mountain, 5 miles so utheast of Orosi There is a higher butte about 0.5 mile east of station. Animals can be ridden to the top, the easiest approach being from the south.

Signal: Cairn on butte.

Station mark: Chiseled circle under center of cairn.

Reference mark: Bronze tablet 3.7 feet east of center of signal. Position of signal computed.

[Latitude $36^{\circ} 31^{\prime} 05.133^{\prime \prime}$. Longitude $119^{\circ} 11^{\prime} 51.445^{\prime \prime}$.]

\begin{tabular}{|c|c|c|c|c|}
\hline Tostation- & Azimuth. & Back azimuth. & Dista & ce. \\
\hline 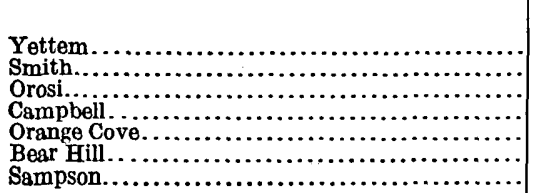 & $\begin{array}{ccc}\circ & \prime & \prime \prime \\
53 & 02 & 53.84 \\
118 & 21 & 33.18 \\
121 & 48 & 11.42 \\
134 & 41 & 00.03 \\
139 & 02 & 30.03 \\
163 & 17 & 56.84 \\
197 & 02 & 59.34\end{array}$ & \begin{tabular}{rcc}
\multicolumn{1}{c}{} & $\prime$ & $\prime \prime$ \\
233 & 01 & 01.97 \\
298 & 16 & 16.63 \\
301 & 45 & 58.30 \\
314 & 33 & 00.41 \\
318 & 58 & 27.37 \\
343 & 14 & 54.32 \\
17 & 06 & 25.17
\end{tabular} & \begin{tabular}{|r|} 
Log. m.eters. \\
3.7677174 \\
4.1765766 \\
3.8158841 \\
4.4484236 \\
4.1886054 \\
4.4217145 \\
4.4649441
\end{tabular} & $\begin{array}{r}\text { Mile. }, \\
3.640 \\
9.331 \\
4.067 \\
17.449 \\
9.593 \\
16.408 \\
18.126\end{array}$ \\
\hline
\end{tabular}

STONE, SAN LUIS OBISPO COUNTY, CAIIF.

A station of the U. S. Coast and Geodetic Survey.

On the higher or eastern one of two conical peaks about 3 miles in a northerly direction from the town of Cayucos. About 15 meters from the station, on the west side of the hill, was a stone fence running across the top of the hill from the northwest to the southeast side. The station was marked by a copper tack in a lead bolt driven into a hole 1 inch in diameter drilled into the solid rock. The reference marks were copper tacks in lead bolts driven into three holes. in the solid rock, 10 inches below surface of ground; to the north, 2 feet 5 inches; to the south, 2 feet 1 inch; to the east, 2 feet 6 inches. Over each was placed a redwood stub 4 by 4 inches, with a copper tack in the top.

[Latitude $35^{\circ} 29^{\prime} 14.760^{\prime \prime}$ (C. G. S.), $35^{\circ} 29^{\prime} 14.732^{\prime \prime}$ (U). Longitude $120^{\circ} 57^{\prime} 00.244^{\prime \prime}$ (C. G. S.), $120^{\circ} 57^{\prime} 00.226^{\prime \prime}$ (U).]

\begin{tabular}{|c|c|c|c|c|}
\hline To station- & Azimuth. & Back azimuth. & Distan & nce. \\
\hline 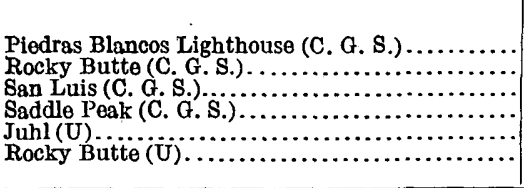 & \begin{tabular}{ccc}
$\circ$ & $\prime$ & \multicolumn{1}{c}{$\prime$} \\
123 & 19 & 09.1 \\
153 & 30 & 12.1 \\
303 & 12 & 04.6 \\
333 & 57 & 13.5 \\
258 & 52 & 18.86 \\
153 & 30 & 12.07 \\
& $\bullet$ &
\end{tabular} & \begin{tabular}{rcc}
\multicolumn{1}{c}{} & \multicolumn{1}{c}{} & \multicolumn{1}{c}{} \\
303 & 07 & 30.4 \\
333 & 26 & 24.1 \\
123 & 25 & 35.4 \\
154 & 02 & 41.8 \\
78 & 56 & 15.06 \\
333 & 26 & 24.10
\end{tabular} & $\begin{array}{c}\text { Log. meters. } \\
4.558071 \\
4.344172 \\
4.626224 \\
4.514292 \\
4.0190373 \\
4.3441912\end{array}$ & 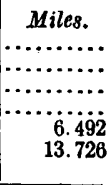 \\
\hline
\end{tabular}

C. G. S. $=$ U. S. Coast and Geodetic Survey in $1883 . \quad$ U $=$ C. F. Urquhart in 1917.

\section{STONE CANYON, MONTEREY COUNTY, CALIF.}

On a prominent brush-covered mountain about 1.5 miles northwest of Stone Canyon coal mine. There is a road under south end of mountain, and animals can be ridden from there to summit. There are higher mountains north and east of this point, any one of which would make as good or a better triangulation station, but none could be plotted on quadrangle to be surveyed.

Signal: A pine tree set in the ground.

Station mark: A triangulation tablet. Position of tablet was computed. 
[Latitude $36^{\circ} 01^{\prime} 30.411^{\prime \prime}$. Longitude $\left.120^{\circ} 33^{\prime} 11.105^{\prime \prime}.\right]$

\begin{tabular}{|c|c|c|c|c|}
\hline To station- & Azimuth. & Back azimuth. & Distan & \\
\hline 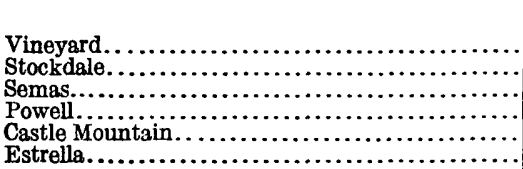 & $\begin{array}{rcc}\circ & \prime & \prime \prime \\
22 & 14 & 41.33 \\
31 & 03 & 02.59 \\
58 & 04 & 18.15 \\
80 & 18 & 40.66 \\
296 & 16 & 20.56 \\
354 & 47 & 22.27\end{array}$ & $\begin{array}{ccc}\circ & \prime & \prime \prime \\
202 & 10 & 27.38 \\
211 & 02 & 03.78 \\
237 & 54 & 46.28 \\
260 & 12 & 53.80 \\
116 & 23 & 51.90 \\
174 & 48 & 31.19\end{array}$ & \begin{tabular}{|c|} 
Log. meters. \\
4.4582886 \\
3.6865267 \\
4.4591241 \\
4.1757593 \\
4.3318977 \\
4.5123908
\end{tabular} & $\begin{array}{r}\text { Mfiles. } \\
17.850 \\
3.019 \\
17.884 \\
9.313 \\
13.343 \\
20.218\end{array}$ \\
\hline
\end{tabular}

SYPHON, MADERA COUNTY, CALIF.

Station is center of windmill tower, on side of ditch, near small lake about 10 miles east of Dos Palos.

[Latitude $36^{\circ} 59^{\prime} 45.419^{\prime \prime}$. Longitude $120^{\circ} 22^{\prime} 20.686^{\prime \prime}$.]

\begin{tabular}{|c|c|c|c|c|}
\hline To station- & Azimuth. & Back azimuth. & Dista & \\
\hline 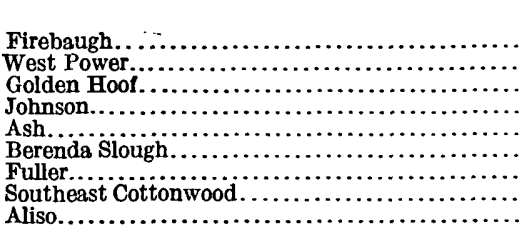 & \begin{tabular}{rcc}
\multicolumn{1}{c}{} & \multicolumn{1}{c}{ ' } \\
26 & 41 & 36.55 \\
46 & 33 & 31.75 \\
68 & 12 & 59.26 \\
121 & 04 & 59.95 \\
176 & 22 & 56.63 \\
238 & 26 & 44.91 \\
267 & 01 & 49.03 \\
300 & 33 & 54.45 \\
339 & 22 & 42.22
\end{tabular} & \begin{tabular}{rcc}
\multicolumn{1}{c}{} & \multicolumn{1}{c}{} & \multicolumn{1}{c}{} \\
206 & 38 & 32.69 \\
226 & 30 & 17.61 \\
248 & 08 & 20.86 \\
300 & 59 & 52.97 \\
356 & 22 & 42.59 \\
58 & 28 & 11.55 \\
87 & 06 & 19.91 \\
120 & 38 & 14.28 \\
159 & 24 & 45.88
\end{tabular} & $\begin{array}{r}\text { Log. meters. } \\
4.2272448 \\
4.0416227 \\
4.0910395 \\
4.1674508 \\
3.9603124 \\
3.6814664 \\
4.0674339 \\
4.0940288 \\
4.1604948\end{array}$ & $\begin{array}{r}\text { Miles. } \\
10.486 \\
6.839 \\
7.663 \\
9.137 \\
5.671 \\
2.984 \\
7.257 \\
7.716 \\
8.992\end{array}$ \\
\hline
\end{tabular}

TALBOT, MADERA COUNTY, CALIF.

On the east side of the Madera-Raymond road about 5 miles north-northeast of Madera.

Signal: Quadripod with cross targets.

Station mark: Iron bench mark centered under signal.

[Latitude $37^{\circ} 02^{\prime} 04.847^{\prime \prime}$. Longitude $120^{\circ} 01^{\prime} 41.670^{\prime \prime}$.]

\begin{tabular}{|c|c|c|c|c|}
\hline To station- & Azimuth. & Back azimuth. & Distar & \\
\hline 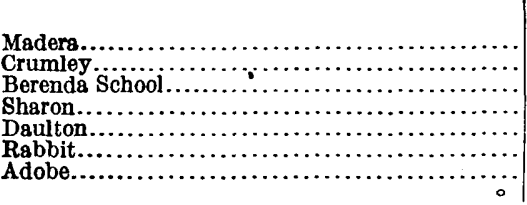 & $\begin{array}{rcc}\circ & \prime & \prime \prime \\
19 & 23 & 39.03 \\
81 & 12 & 31.13 \\
91 & 50 & 47.80 \\
128 & 38 & 54.10 \\
200 & 28 & 29.77 \\
217 & 24 & 27.28 \\
270 & 09 & 07.42\end{array}$ & \begin{tabular}{rcc}
$\circ$ & \multicolumn{1}{c}{,} \\
199 & 22 & 28.17 \\
261 & 07 & 30.62 \\
271 & 46 & 17.76 \\
308 & 35 & 11.51 \\
20 & 30 & 08.46 \\
37 & 27 & 59.14 \\
90 & 15 & 09.30
\end{tabular} & \begin{tabular}{r|} 
Log. meters. \\
3.9431201 \\
4.0963184 \\
4.0447607 \\
4.0672925 \\
4.0625406 \\
4.1545450 \\
4.1717116
\end{tabular} & $\begin{array}{r}\text { Miles. } \\
\mathbf{5 . 4 5 1} \\
\mathbf{7 . 7 5 7} \\
\mathbf{6 . 8 8 8} \\
7.255 \\
\mathbf{7 . 1 7 6} \\
\mathbf{8 . 8 6 9} \\
\mathbf{9 . 2 2 7}\end{array}$ \\
\hline
\end{tabular}

TANES, FRESNO COUNTY, CALIF. (Not occupied.)

Standard Oil Co.'s Mendota pump station.

Station mark: Center of tank house.

[Latitude $36^{\circ} 37^{\prime} 53.686^{\prime \prime}$. Longitude $120^{\circ} 18^{\prime} 43.816^{\prime \prime}$.]

\begin{tabular}{|c|c|c|c|c|}
\hline To station- & Azimuth. & Back azimuth. & Dista1 & ce. \\
\hline 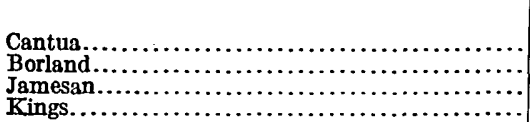 & \begin{tabular}{rcc}
\multicolumn{1}{c}{$\cdot$} & $\prime \prime$ \\
23 & 04 & 37.47 \\
164 & 36 & 40.68 \\
220 & 05 & 19.05 \\
232 & 40 & 58.36
\end{tabular} & $\begin{array}{rcc}\circ & , & \prime \prime \\
203 & 02 & 22.53 \\
344 & 36 & 06.12 \\
40 & 09 & 07.99 \\
52 & 43 & 59.33\end{array}$ & $\begin{array}{c}\text { Log. meters. } \\
4.1576810 \\
3.7336757 \\
4.1732567 \\
3.9810611\end{array}$ & $\begin{array}{r}\text { Miles. } \\
8.934 \\
3.365 \\
9.260 \\
5.949\end{array}$ \\
\hline
\end{tabular}


TELEPHONE, FRESNO COUNTY, CAIIF.

The windmill derrick at the Miller \& Lux Co.'s sheep camp, known as the Telephone ranch, 10 miles from Mendota, a town on the Southern Pacific R. $\mathbb{R}$., and 0.5 mile north of the Idria-Mendota road. The Chaney ranch is 4 miles west on same road and is the only ranch of importance between Mendota and Idria. Hay and stock water can be had at the Telephone ranch, but no water fit for drinking.

Station mark: The pipe extending into ground.

[Latitude $36^{\circ} 41^{\prime} 30.991^{\prime \prime}$. Longitude $120^{\circ} 30^{\prime} 52.018^{\prime \prime}$.]

\begin{tabular}{|c|c|c|c|c|}
\hline To station- & Azimuth. & Back arimuth. & Diste & \\
\hline 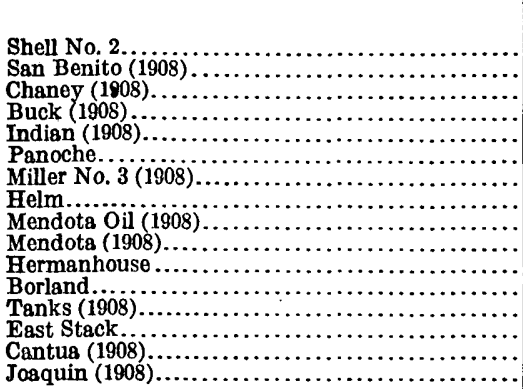 & $\begin{array}{rrc}\circ & \prime & \prime \prime \\
2 & 01 & 03.04 \\
18 & 01 & 42.54 \\
52 & 58 & 34.39 \\
63 & 08 & 46.06 \\
73 & 24 & 41.49 \\
99 & 39 & 44.38 \\
150 & 42 & 19.89 \\
226 & 08 & 43.25 \\
227 & 57 & 46.84 \\
242 & 28 & 40.44 \\
251 & 20 & 44.18 \\
275 & 00 & 53.67 \\
290 & 16 & 02.85 \\
315 & 29 & 52.69 \\
327 & 55 & 15.38 \\
353 & 02 & 07.91\end{array}$ & $\begin{array}{rcc}\circ & \prime & \prime \prime \\
182 & 00 & 53.54 \\
197 & 57 & 04.89 \\
232 & 56 & 20.56 \\
212 & 55 & 57.09 \\
253 & 17 & 06.26 \\
279 & 30 & 47.63 \\
330 & 40 & 42.19 \\
46 & 13 & 39.50 \\
48 & 01 & 57.80 \\
62 & 33 & 47.13 \\
71 & 27 & 51.79 \\
95 & 07 & 34.13 \\
110 & 23 & 17.63 \\
135 & 33 & 47.10 \\
148 & 00 & 14.53 \\
173 & 04 & 07.98\end{array}$ & \begin{tabular}{|c} 
Log. meters. \\
4.0508452 \\
4.5748389 \\
3.8432915 \\
4.5555836 \\
4.2957845 \\
4.3541563 \\
3.9181290 \\
4.2310930 \\
4.1464244 \\
4.1567673 \\
4.2724558 \\
4.2229194 \\
4.2852279 \\
4.1438113 \\
4.3712011 \\
4.6182998
\end{tabular} & $\begin{array}{r}\text { Miles. } \\
6.985 \\
23.345 \\
4.332 \\
22.332 \\
12.278 \\
14.045 \\
5.146 \\
10.579 \\
\ldots \ldots . . . \\
\ldots \ldots . .36 \\
11.636 \\
10.382 \\
\ldots . . .65 \\
8.653 \\
14.607 \\
25.802\end{array}$ \\
\hline
\end{tabular}

TIERRA LOMA, FRESTO COUNTY, CALIF.

Station is windmill at the Tierra Loma School, 20 miles south of Dos Palos.

Signal: Center of mill.

Station mark: Center of well.

[Latitude $36^{\circ} 43^{\prime} 40.905^{\prime \prime}$. Longitude $120^{\circ} 39^{\prime} 27.069^{\prime \prime}$.]

\begin{tabular}{|c|c|c|c|c|}
\hline To station- & Azimuth. & Back azimuth. & Dista & \\
\hline 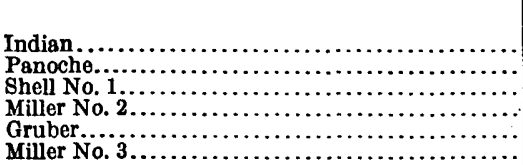 & \begin{tabular}{rcc}
\multicolumn{1}{c}{$\circ$} & \multicolumn{1}{c}{} & \multicolumn{1}{c}{} \\
32 & 26 & 21.73 \\
88 & 35 & 06.15 \\
153 & 33 & 33.21 \\
184 & 50 & 59.61 \\
211 & 43 & 38.63 \\
249 & 43 & 47.76
\end{tabular} & $\begin{array}{rcc}\circ & \prime & \prime \prime \\
212 & 23 & 54.00 \\
268 & 31 & 17.18 \\
333 & 32 & 32.07 \\
4 & 41 & 24.67 \\
31 & 44 & 36.66 \\
69 & 47 & 18.14\end{array}$ & $\begin{array}{c}\text { Log. meters. } \\
4.0588652 \\
3.9779082 \\
3.7552258 \\
4.1038132 \\
3.6604077 \\
\mathbf{3 . 9 6 8 4 4 3 7}\end{array}$ & $\begin{array}{r}\text { Miles. } \\
\mathbf{7 . 1 1 6} \\
\mathbf{5 . 9 0 6} \\
\mathbf{3 . 5 3 7} \\
\mathbf{7 . 8 9 2} \\
\mathbf{2 . 8 4 3} \\
\mathbf{5 . 7 7 8}\end{array}$ \\
\hline
\end{tabular}

TIVEY, FRESNO COUNTY, CALIF. (Not occupled.)

[Latitude $36^{\circ} 44^{\prime} 35.794^{\prime \prime}$. Longitude $119^{\circ} 23^{\prime} 42.786^{\prime \prime}$.]

\begin{tabular}{|c|c|c|c|c|}
\hline To station- & Azimuth. & Back azimuth. & Dista & se. \\
\hline 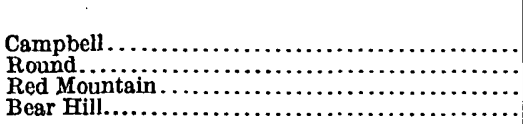 & \begin{tabular}{rcc}
\multicolumn{1}{c}{} & \multicolumn{1}{c}{,$\prime$} \\
23 & 40 & 16.25 \\
129 & 33 & 28.55 \\
178 & 31 & 15.14 \\
268 & 15 & 01.33
\end{tabular} & \begin{tabular}{rcc}
\multicolumn{1}{c}{} & $\prime$ & $\prime \prime$ \\
203 & 39 & 20.67 \\
309 & 29 & 22.15 \\
358 & 31 & 06.83 \\
88 & 19 & 03.89
\end{tabular} & $\begin{array}{r}\text { Log. meters. } \\
\text { 3. } 7595096 \\
\text { 4. } 1215523 \\
\text { 4. } 1244156 \\
\text { 4. } 0027310\end{array}$ & $\begin{array}{r}\text { Miles. } \\
\text { 3. } 572 \\
8.221 \\
8.275 \\
6.253\end{array}$ \\
\hline
\end{tabular}

TORO MOUNT, MONTEREY COUNTY, CALIF.

A station of the U. S. Coast and Geodetic Survey.

On the untimbered range of mountains bordering the Salinas Valley on the west. on the smooth, grassy hill about 500 yards south of A. B. Parson's house and on his land. It is easily reached from Salinas City and is in full view of the town.

Station mark: A copper bolt set in a flat-topped rock 2 feet beneath the surface of the ground, and a cross in copper bolt in top of pier built of concrete and topped with brick and cement. Two other piers are standing northwest of the station. 
[Latitude $36^{\circ} 31^{\prime} 34.712^{\prime \prime}$ (B., C. G. S.), $36^{\circ} 31^{\prime} 34.742^{\prime \prime}$ (C. G. S. *) Longitude $121^{\circ} 36^{\prime} 32.276^{\prime \prime}$ (B., C. G S.), $121^{\circ} 36^{\prime} 32.284^{\prime \prime}$ (C..G.S. $*$ ).]

\begin{tabular}{|c|c|c|c|c|}
\hline Tostation- & Azimuth. & Back azimuth. & Distar & nce. \\
\hline 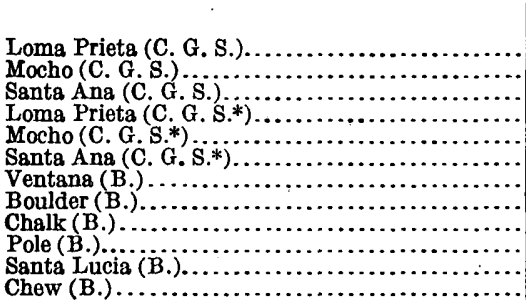 & \begin{tabular}{rcc}
\multicolumn{1}{c}{} & $\prime$ & $\prime \prime$ \\
162 & 12 & 31.32 \\
182 & 34 & 42.13 \\
218 & 31 & 04.10 \\
162 & 12 & 32.85 \\
182 & 34 & 42.61 \\
218 & 31 & 06.92 \\
12 & 57 & 22.61 \\
45 & 53 & 42.90 \\
100 & 44 & 39.43 \\
321 & 45 & 48.39 \\
337 & 52 & 09.62 \\
351 & 09 & 35.40
\end{tabular} & $\begin{array}{rcc}\circ & \prime & \prime \prime \\
342 & 04 & 05.43 \\
2 & 36 & 38.58 \\
38 & 44 & 33.91 \\
342 & 04 & 06.98 \\
2 & 36 & 39.07 \\
38 & 44 & 36.72 \\
192 & 54 & 55.91 \\
225 & 47 & 20.74 \\
280 & 39 & 27.34 \\
141 & 50 & 00.10 \\
157 & 58 & 56.29 \\
171 & 11 & 03.72\end{array}$ & $\begin{array}{r}\text { Log. meters. } \\
4.8338624 \\
5.0240270 \\
4.7311660 \\
4.8338515 \\
5.0240231 \\
4.7311612 \\
4.4395357 \\
4.3487418 \\
4.1228971 \\
4.2317425 \\
4.6584603 \\
4.3829144\end{array}$ & 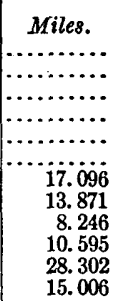 \\
\hline
\end{tabular}

(C. G. S.) $=$ U. S. Coast and Geodetic Survey in 1885 , (C. G. S.*)=U. S. Coast and Geodetic Survey in $1906,(B)=$. L. F. Biggs in 1917.

UNDERWOOD, MERCED COUNTY, CALIF. (Not occupied.)

Windmill derrick at the Miller \& Lux Underwood ranch, about 7 miles east of Ingomar on the Southern Pacific R. R.

[Latitude $37^{\circ} 11^{\prime} 32.236^{\prime \prime}$. Longitude $120^{\circ} 50^{\prime} 19.483^{\prime \prime}$.]

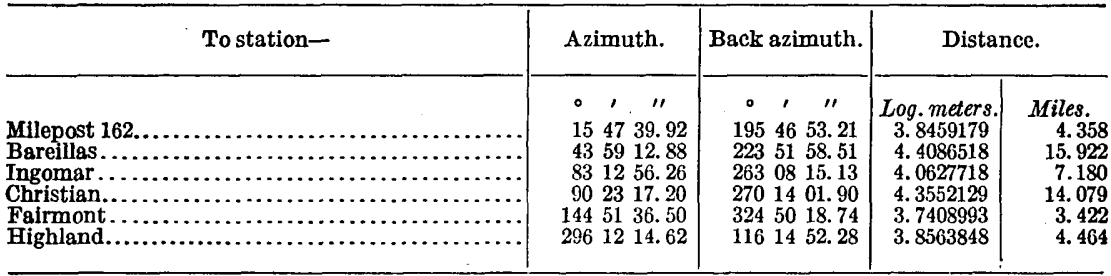

VENTANA, MONTEREY COUNTY, CALIF. (Not occupled.)

Summit of sharp peak near the Ventanos.

[Latitude $36^{\circ} 17^{\prime} 04.765^{\prime \prime}$. Longitude $121^{\circ} 40^{\prime} 39.456^{\prime \prime}$.]

\begin{tabular}{|c|c|c|c|c|}
\hline To station- & Azimuth. & Back azimuth. & \multicolumn{2}{|c|}{ Distance. } \\
\hline 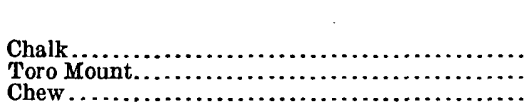 & $\begin{array}{ccc}\circ & \prime & \prime \prime \\
166 & 45 & 29.88 \\
192 & 54 & 55.91 \\
253 & 20 & 14.38\end{array}$ & $\begin{array}{rcc}\circ & \prime & \prime \prime \\
346 & 42 & 44.72 \\
12 & 57 & 22.61 \\
73 & 24 & 08.77\end{array}$ & \begin{tabular}{|r|} 
Log. meters. \\
4.4782974 \\
4.4395357 \\
4.0132896
\end{tabular} & $\begin{array}{r}\text { Miles. } \\
18.692 \\
17.096 \\
6.407\end{array}$ \\
\hline
\end{tabular}

VINEYARD, SAN LUIS OBISPO COUNTY, CALIF.

On the highest point of a bald ridge 3 miles northeast of San Miguel, a town on the Southern Pacific R. R. (Coast Line). It can be reached on horseback from almost any direction, but the best route from San Miguel is by way of Mahony Canyon. Cross the Salinas River on bridge at San Miguel; on east side of river there are three roads, take the middle one and go uphill and along this road to first road turning to left. This road goes around corner of fence and downhill to Mahony Canyon and up the canyon passing through a gate. After passing through this gate go 0.25 mile along road to first gate in fence to the west or left hand; go through this gate and up ridge to summit.

Signal: An oak tree set in the ground.

Station mark: A triangulation tablet set in rock 0.94 meter northeast of signal. Position of tablet was computed. Azimuth from tablet to signal, $50^{\circ} 16^{\prime}$; distance, 0.94 meter. 
[Latitude $35^{\circ} 47^{\prime} 07.497^{\prime \prime}$. Longitude $120^{\circ} 40^{\prime} 24.133^{\prime \prime}$.]

\begin{tabular}{|c|c|c|c|c|}
\hline To station- & Azimuth. & Back azimuth. & DistaI & \\
\hline 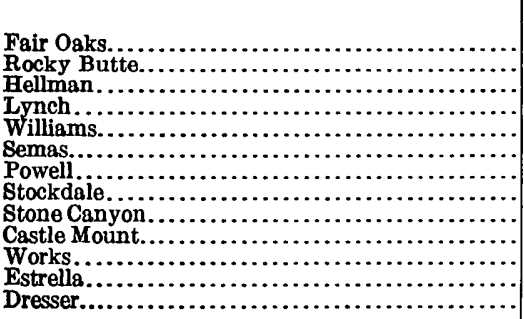 & \begin{tabular}{rcc}
\multicolumn{1}{c}{} & $\prime$ & $\prime \prime$ \\
34 & 45 & 14.03 \\
69 & 14 & 41.10 \\
84 & 37 & 24.55 \\
86 & 55 & 03.93 \\
122 & 03 & 50.88 \\
129 & 55 & 01.36 \\
170 & 43 & 30.24 \\
200 & 23 & 36.85 \\
202 & 10 & 27.38 \\
240 & 22 & 33.77 \\
255 & 44 & 12.49 \\
292 & 44 & 00.56 \\
337 & 54 & 46.84
\end{tabular} & \begin{tabular}{rcc}
\multicolumn{1}{c}{} & $\prime$ & $\prime \prime$ \\
214 & 41 & 01.53 \\
249 & 01 & 10.69 \\
264 & 34 & 04.51 \\
266 & 44 & 07.46 \\
301 & 52 & 19.91 \\
309 & 49 & 44.67 \\
350 & 41 & 58.26 \\
20 & 26 & 52.04 \\
22 & 14 & 41.33 \\
60 & 34 & 17.50 \\
75 & 46 & 58.59 \\
112 & 49 & 22.33 \\
157 & 58 & 18.82
\end{tabular} & $\begin{array}{r}\text { Log. meters. } \\
4.2808062 \\
4.5720691 \\
3.9360395 \\
4.4509332 \\
4.5424997 \\
4.2476951 \\
4.3870387 \\
4.3790687 \\
4.4582886 \\
4.5395326 \\
3.8667339 \\
4.1761640 \\
4.3861901\end{array}$ & $\begin{array}{r}\text { Miles. } \\
11.862 \\
23.196 \\
5.363 \\
17.550 \\
21.670 \\
10.991 \\
15.149 \\
14.874 \\
17.850 \\
21.522 \\
4.572 \\
9.322 \\
1.5 .120\end{array}$ \\
\hline
\end{tabular}

WEST BUTTE, MARIPOSA COUNTY, CALIF.

On the west one of three buttes on the east edge of the San Joaquin Valley, 3 miles north of the Grade ranch, and about 20 miles northeast of Merced. It can be reached by following the road east from Merced to Tuttle, a station on the Atchison, Topeka \& Santa $\mathrm{Fe}$ Ry., thence 1 mile north to Bear Creek bridge, thence along the main road on the south side of Bear Creek to a point where the road turns to the Grade ranch; thence by the ranch through pastures to the point.

Signal: A tree braced by poles and rock on highest point of butte.

Station mark: A triangulation tablet set in rock under center of tree.

[Latitude $37^{\circ} 25^{\prime} 14.8200^{\prime \prime}$ Longitude $120^{\circ} 13^{\prime} 58.266^{\prime \prime}$.

\begin{tabular}{|c|c|c|c|c|}
\hline To station- & Azimuth. & Back azimuth. & Dista & \\
\hline 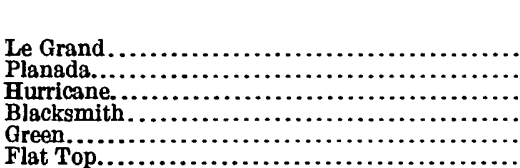 & $\begin{array}{rcc}\circ & \prime & \prime \prime \\
2 & 41 & 24.74 \\
28 & 52 & 54.28 \\
50 & 40 & 18.46 \\
83 & 45 & 34.87 \\
308 & 37 & 06.40 \\
326 & 40 & 51.91\end{array}$ & $\begin{array}{ccc}\circ & \prime & \prime \prime \\
182 & 41 & 05.26 \\
208 & 49 & 36.32 \\
230 & 36 & 39.87 \\
263 & 40 & 02.21 \\
128 & 45 & 55.93 \\
146 & 45 & 19.51\end{array}$ & \begin{tabular}{|r|} 
Log. meters. \\
4.2268546 \\
4.2211092 \\
4.0589318 \\
4.1317959 \\
4.4397964 \\
4.2963446
\end{tabular} & $\begin{array}{r}\text { Miles. } \\
10.476 \\
10.339 \\
7.117 \\
8.417 \\
17.106 \\
12.294\end{array}$ \\
\hline
\end{tabular}

WEST POWER, FRESNO COUNTY, CALIF.

The west one of two high poles that convey the power line across San Joaquin River at a point 5 miles north of Firebaugh. The pole leans slightly, and the cross arm about 15 feet from top was used.

Signal and station mark: The power pole.

Reference mark: Iron bench mark set at northeast corner of a warehouse, 14.6 feet from pole. Position of pole computed. Azimuth from bench mark to signal, $178^{\circ}$ $23^{\prime}$; distance, 14.6 feet.

[ Latitude $36^{\circ} 55^{\prime} 39.800^{\prime \prime}$. Longitude $120^{\circ} 27^{\prime} 43.566^{\prime \prime}$.]

\begin{tabular}{|c|c|c|c|c|}
\hline To station- & Azimuth. & Back azimuth. & Dista & \\
\hline 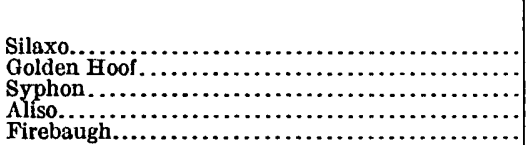 & $\begin{array}{rcc}\circ & \prime & \prime \prime \\
47 & 29 & 54.29 \\
130 & 47 & 11.12 \\
226 & 30 & 17.61 \\
294 & 29 & 16.98 \\
356 & 48 & 59.36\end{array}$ & \begin{tabular}{rcc}
\multicolumn{1}{c}{} & $\prime$ & $\prime \prime$ \\
227 & 27 & 28.48 \\
310 & 45 & 46.99 \\
46 & 33 & 31.75 \\
114 & 34 & 34.41 \\
176 & 49 & 09.48
\end{tabular} & \begin{tabular}{|c|} 
Log. meters. \\
3.9114933 \\
3.6603027 \\
4.0416227 \\
4.1579846 \\
3.8762201
\end{tabular} & $\begin{array}{r}\text { Miles. } \\
5.068 \\
2.842 \\
6.839 \\
8.940 \\
4.673\end{array}$ \\
\hline
\end{tabular}

WHITE, MERCED COUNTY, CALIF.

On a bald plateau about 4 miles southwest of Miller \& Lux Co.'s Jamison ranch, which is on the westernmost canal and 9 miles south of Los Banos. Station is about 11 miles by road west of South Dos Palos. The signal is on a smooth, grass-covered hill witk no rocks on it. There is a point a little higher about 0.25 mile south. Animals 
can be ridden to point, and a light wagon may be driven to it, though the hill is rather steep.

Signal: A 4 by 4-inch pole reaching 12 feet above ground and bearing two targets at right angles to each other.

Station mark: Triangulation tablet in rock placed in ground 0.65 meter northwest of signal. The rock is 6 inches above ground on the northwest side of tablet.

[Latitude $36^{\circ} 54^{\prime} 48.435^{\prime \prime}$. Longitude $120^{\circ} 50^{\prime} 27.300^{\prime \prime}$.]

\begin{tabular}{|c|c|c|c|c|}
\hline Tostation- & Azimuth. & Back azimuth. & Distar & e. \\
\hline 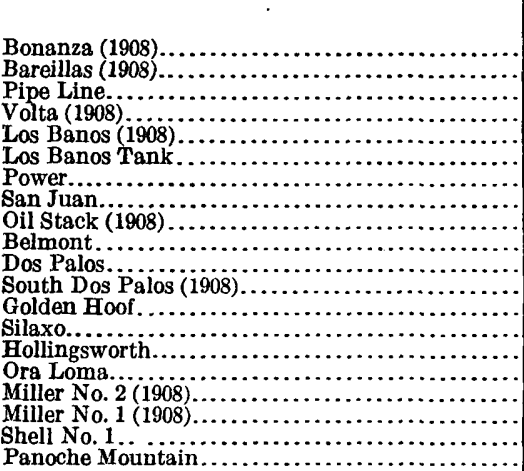 & $\begin{array}{rcc}\circ & \prime & \prime \prime \\
46 & 16 & 03.78 \\
125 & 23 & 37.82 \\
143 & 40 & 46.98 \\
158 & 53 & 48.87 \\
177 & 14 & 08.56 \\
178 & 39 & 32.25 \\
214 & 36 & 06.97 \\
221 & 24 & 03.49 \\
234 & 22 & 12.82 \\
234 & 35 & 06.76 \\
246 & 50 & 14.77 \\
251 & 12 & 05.25 \\
261 & 18 & 55.45 \\
277 & 57 & 52.34 \\
281 & 09 & 40.43 \\
287 & 12 & 20.40 \\
294 & 25 & 41.90 \\
295 & 07 & 03.74 \\
318 & 11 & 05.45 \\
341 & 42 & 12.49\end{array}$ & \begin{tabular}{rcc}
\multicolumn{1}{c}{$\bullet$} &, & $\prime \prime$ \\
226 & 11 & 02.36 \\
305 & 16 & 29.54 \\
323 & 36 & 59.72 \\
338 & 50 & 39.26 \\
357 & 13 & 49.50 \\
358 & 39 & 22.92 \\
34 & 40 & 31.53 \\
41 & 30 & 42.69 \\
54 & 27 & 05.63 \\
54 & 42 & 58.16 \\
66 & 58 & 01.42 \\
71 & 18 & 47.80 \\
81 & 31 & 10.80 \\
98 & 09 & 05.30 \\
101 & 17 & 06.20 \\
107 & 17 & 06.15 \\
114 & 32 & 54.23 \\
115 & 10 & 22.20 \\
138 & 16 & 40.08 \\
161 & 44 & 58.70
\end{tabular} & $\begin{array}{r}\text { Log. meters. } \\
4.2364938 \\
4.3343198 \\
4.1978358 \\
4.3346184 \\
4.2102514 \\
4.2140071 \\
4.2818150 \\
4.3940425 \\
4.1708242 \\
4.3759685 \\
4.3197460 \\
4.2431390 \\
4.4861133 \\
4.4476319 \\
4.2727986 \\
4.0912602 \\
4.2811679 \\
3.9562094 \\
4.3171585 \\
4.3408881\end{array}$ & $\begin{array}{r}\text { Miles: } \\
10.711 \\
13.417 \\
9.799 \\
13.427 \\
10.083 \\
10.171 \\
11.890 \\
15.396 \\
9.208 \\
14.768 \\
12.975 \\
10.876 \\
19.031 \\
17.417 \\
11.645 \\
7.667 \\
11.872 \\
5.618 \\
12.898 \\
13.622\end{array}$ \\
\hline
\end{tabular}

WILLIAMS, MONTEREY COUNTY, CALIF.

Station built by L. F. Biggs in October, 1916. Signal is on highest point of a mountain, locally known as Williams Mountain. It can be reached from Lockwood, a post office, hotel, and store in the San Antonio Valley. Go due east from Lockwood about 1.5 miles or 2 miles, passing through two gates, thence northeast on west side of a plowed field about 0.75 mile and through another gate. This is as far as a.wagon can be taken. From this gate there is a good trail, known as the horse trail, going east and southeast to canyon at foot of mountain. Before reaching canyon a gate is passed through a one-wire fence; when canyon at foot of mountain is reached go down it about 100 yards and through another gate: thence up ridge on south side of summit

Signal: A pine tree set in ground about 2 feet and guyed with wire.

Station mark: A triangulation tablet in rock. The original mark was too near the tree to set the instrument over it, so it was moved 0.86 meter about east of tree. Signal was not changed though it was dropped 2 feet in the ground to make it more secure. Position of tablet was computed. Azimuth from tablet to signal, $99^{\circ} 19$; distance, 0.86 meter.

[Latitude $35^{\circ} 57^{\prime} 06.577^{\prime \prime}$. Longitude $121^{\circ} 00^{\prime} 03.402^{\prime \prime}$.]

\begin{tabular}{|c|c|c|c|c|}
\hline To station- & Azimuth. & Back azimuth. & Dista & \\
\hline 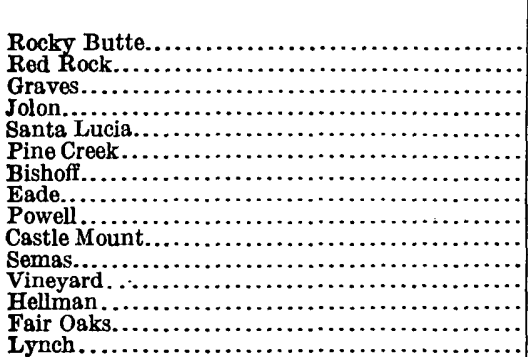 & \begin{tabular}{rcc}
\multicolumn{1}{c}{} & \multicolumn{1}{c}{ ' } \\
9 & 22 & 58.39 \\
33 & 33 & 06.35 \\
48 & 45 & 24.83 \\
102 & 30 & 49.89 \\
119 & 55 & 10.96 \\
129 & 29 & 45.23 \\
145 & 54 & 59.25 \\
185 & 22 & 32.97 \\
257 & 35 & 27.71 \\
271 & 08 & 40.76 \\
293 & 56 & 33.17 \\
301, & 52 & 19.91 \\
312 & 28 & 40.07 \\
331 & 10 & 27.19 \\
355 & 57 & 05.89
\end{tabular} & \begin{tabular}{rcc}
\multicolumn{1}{c}{} & $\prime$ & $\prime \prime$ \\
189 & 20 & 56.31 \\
213 & 27 & 31.69 \\
228 & 41 & 05.58 \\
282 & 23 & 51.69 \\
299 & 40 & 26.76 \\
309 & 21 & 44.89 \\
325 & 47 & 57.01 \\
5 & 23 & 40.36 \\
77 & 45 & 28.32 \\
91 & 31 & 58.16 \\
114 & 02 & 47.66 \\
122 & 03 & 50.88 \\
132 & 36 & 50.54 \\
151 & 17 & 43.97 \\
175 & 57 & 38.95
\end{tabular} & $\begin{array}{r}\text { Log. meters. } \\
\text { 4. } 5077331 \\
\text { 4. } 4145015 \\
\text { 4. } 1687794 \\
\text { 4. } 2617385 \\
\text { 4. } 6368799 \\
\text { 4. } 4227866 \\
4.5047955 \\
4.4842617 \\
4.4186037 \\
\text { 4. } 7758227 \\
4.2434984 \\
4.5424997 \\
4.4549712 \\
4.5906538 \\
4.3027095\end{array}$ & $\begin{array}{r}\text { Miles. } \\
20.002 \\
16.138 \\
9.165 \\
11.352 \\
26.930 \\
16.449 \\
19.868 \\
18.950 \\
16.291 \\
37.083 \\
10.886 \\
21.670 \\
17.714 \\
24.210 \\
12.476\end{array}$ \\
\hline
\end{tabular}


WORES. MONTEREY COUTTY, CALIF.

On ridge 9 miles east of San Miguel, on land owned by Mr. Works; F. E. Lowe's Estate ranch house is $\mathbf{0 . 7 5}$ mile northward from signal. From San Miguel cross river and take middle one of three roads (signboard "Parkfield via Hog Canyon"); follow this road eastward and turn up the canyon at a schoolhouse at forks in road, at a sharp bend in road where there is a signboard reading "San Miguel 9 miles, Parkfield 17 miles." Go through a gate and follow road northward 0.5 mile from where a deserted cabin can be seen 0.5 mile westward. The signal is on a ridge 0.25 mile west of this cabin and 1 mile northwest of point where main road was left.

Signal: Timber structure with white target.

Station mark: Bronze tablet set in rock flush with ground under signal. Position of tablet computed.

[Latitude $35^{\circ} 48^{\prime} 06.221^{\prime \prime}$. Longitude $120^{\circ} 35^{\prime} 40.136^{\prime \prime}$.]

\begin{tabular}{|c|c|c|c|c|}
\hline To station- & Azimuth. & Back azimuth. & Distar & \\
\hline 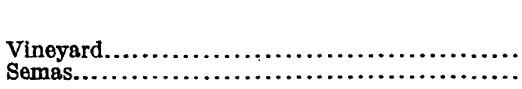 & $\begin{array}{rcc}\circ & \prime & \prime \prime \\
75 & 46 & 58.59 \\
114 & 47 & 13.46\end{array}$ & $\begin{array}{ccc}\circ & \prime & \prime \prime \\
255 & 44 & 12.49 \\
294 & 39 & 10.40\end{array}$ & $\begin{array}{r}\text { Log. meters. } \\
3.8667339 \\
4.3577320\end{array}$ & $\begin{array}{r}\text { Miles. } \\
4.572 \\
14.161\end{array}$ \\
\hline
\end{tabular}

YETTEM, FRESNO COUNTY, CALIF. (Not occupled.)

Station mark: Cupola on schoolhouse at Yettem crossroads.

[Latitude $36^{\circ} 29^{\prime} 10.856^{\prime \prime}$. Longitude $119^{\circ} 14^{\prime} 59.502^{\prime \prime}$.]

\begin{tabular}{|c|c|c|c|c|}
\hline To station- & Azimuth. & Back azimuth. & Dista & \\
\hline 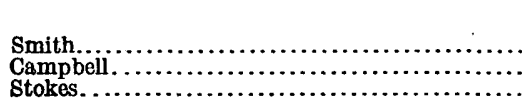 & $\begin{array}{ccc}\circ & \prime & \prime \prime \\
141 & 16 & 31.00 \\
146 & 40 & 01.73 \\
233 & 01 & 01.97\end{array}$ & \begin{tabular}{rcc}
\multicolumn{1}{c}{} & $\prime$ & $\prime \prime$ \\
321 & 13 & 06.53 \\
326 & 33 & 54.39 \\
53 & 02 & 53.84
\end{tabular} & $\begin{array}{c}\text { Log. meters. } \\
4.1351443 \\
4.4446605 \\
3.7677174\end{array}$ & $\begin{array}{r}\text { Miles. } \\
8.482 \\
17.299 \\
3.640\end{array}$ \\
\hline
\end{tabular}

Bonanza, Carrisalito Springs, Center School, Ortignlita, Panoche, and Tumey Hills quadrangles.

FRESNO AND MERCED COUNTIES.

In the summer of $1919 \mathrm{~L}$. F. Biggs extended triangulation over the above-named quadrangles. Twelve points were located, based on stations Bonanza and White, North American datum.

BONANZA, MERCED COUNTY, CALIF.

On a high bald peak, on which oak trees are growing just below the summit on the north side, about 3 miles northeast of Bonanza post office. A road leads to Bonanza from Tres Pinos on the Southern Pacific R. R. (distance 24 miles); thence up canyon 1 mile to Mr. Sepulveda's ranch. From this place follow trail to summit. Animals can be ridden all the way up. Station can also be reached from San Joaquin side by going up a wood road to summit, thence along ridge to highest point.

Signal and station mark: A cairn 8 feet high and triangulation tablet in rock placed under its center.

[Latitude $36^{\circ} 48^{\prime} 21.558^{\prime \prime}$. Longitude $120^{\circ} 58^{\prime} 49.786^{\prime \prime}$.]

\begin{tabular}{|c|c|c|c|c|}
\hline Tostation- & Azimuth. & Back azimuth. & Dista1 & ce. \\
\hline 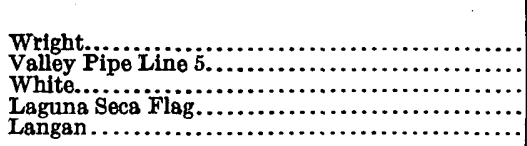 & $\begin{array}{ccc}\circ & \prime & \prime \prime \\
178 & 45 & 46.10 \\
205 & 30 & 04 \\
226 & 11 & 02.36 \\
240 & 31 & 13.02 \\
244 & 54 & 19.46\end{array}$ & \begin{tabular}{rcc}
\multicolumn{1}{c}{$\cdot$} & \multicolumn{1}{c}{$\cdot 1$} \\
358 & 45 & 37.10 \\
25 & 33 & 36 \\
46 & 16 & 03.78 \\
60 & 36 & 04.37 \\
64 & 57 & 44.68
\end{tabular} & \begin{tabular}{|c|} 
Log. meters. \\
4.2551166 \\
4.30818 \\
4.2364938 \\
4.1407282 \\
3.9718627
\end{tabular} & $\begin{array}{r}\text { Miles. } \\
10.677 \\
12.634 \\
10.711 \\
8.592 \\
5.824\end{array}$ \\
\hline
\end{tabular}


CARRISALITO, MERCED COUNTY, CAIIF.

On high, dome-shaped hill just west of the Carrisalito ranch, owned by Arbura Bros. There is a growth of cedar trees on north slope of hill.

Station and signal: A rock monument with tree in center.

Reference mark: $\Lambda$ tablet 1 foot east of signal.

[Latitude $36^{\circ} 52^{\prime} 02.22^{\prime \prime}$. Longitude $121^{\circ} 01^{\prime} 51.36^{\prime \prime}$.]

\begin{tabular}{|c|c|c|c|c|}
\hline Tostation- & Azimuth. & Back azimuth. & Dista & ce. \\
\hline 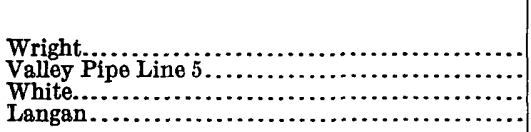 & $\begin{array}{ccc}\circ & \prime \prime & \prime \prime \\
201 & 39 & 22.46 \\
228 & 53 & 55 \\
253 & 06 & 47.04 \\
282 & 15 & 24.00\end{array}$ & 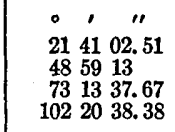 & \begin{tabular}{|c} 
Log. meters. \\
4.0479295 \\
4.24494 \\
4.2478847 \\
4.1235201
\end{tabular} & $\begin{array}{r}\text { Miles. } \\
6.939 \\
10.922 \\
10.996 \\
8.258\end{array}$ \\
\hline
\end{tabular}

CHANEY, FRESNO COUNTY, CALIF.

The windmill derrick at the Chaney ranch, 14 miles from Mendota, on the MendotaIdria road. This is the only ranch of importance on this road between Mendota and Idria. There is sufficient hay and water here, though drinking water has to be hauled from Mendota. The ranch is owned by the Hollister Co. and is sometimes called the Hollister ranch.

Station mark: The pipe extending into ground.

[Latitude $36^{\circ} 39^{\prime} 14.758^{\prime \prime}$.. Longitude $120^{\circ} 34^{\prime} 36.094^{\prime \prime}$.]

\begin{tabular}{|c|c|c|c|c|}
\hline Tostation- & Azimuth. & Back azimuth. & Dista & ce. \\
\hline 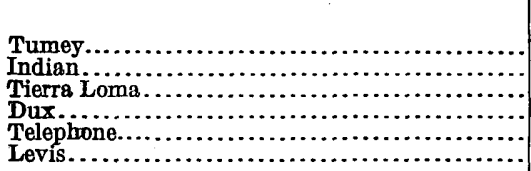 & $\begin{array}{rll}\circ & \prime & \prime \prime \\
29 & 03 & 53 \\
83 & 46 & 01 \\
138 & 35 & 46 \\
189 & 48 & 28 \\
232 & 56 & 21 \\
282 & 34 & 57\end{array}$ & \begin{tabular}{rcc}
\multicolumn{1}{c}{} & $\prime$ & $\prime \prime$ \\
209 & 00 & 54 \\
263 & 42 & 39 \\
318 & 36 & 48 \\
9 & 46 & 28 \\
52 & 58 & 34 \\
102 & 41 & 05
\end{tabular} & $\begin{array}{r}\text { Log. meters. } \\
4.17760 \\
4.12871 \\
4.03864 \\
4.19534 \\
3.84329 \\
4.19361\end{array}$ & $\begin{array}{r}\text { Miles. } \\
9.352 \\
8.357 \\
6.792 \\
9.743 \\
4.332 \\
9.704\end{array}$ \\
\hline
\end{tabular}

DUX, FRESNO COUNTY, CALIF. (Not occupled.)

Station is a high derrick of a well, 3.0 miles south of Miller \& Lux sheep ranch, which is about 5 miles southwest from Firebaugh.

Signal: The derrick.

Station mark: Pipe in well.

[Latitude $36^{\circ} 47^{\prime} 36.06^{\prime \prime}$. Longitude $120^{\circ} 32^{\prime} 48.94^{\prime \prime}$.]

\begin{tabular}{|c|c|c|c|c|}
\hline Tostation- & Azimuth. & Back azimuth. & Distar & $c \theta$. \\
\hline 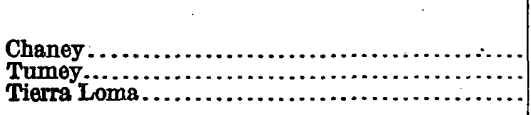 & $\begin{array}{rcc}\circ & \prime & \prime \prime \\
9 & 46 & 28 \\
19 & 13 & 44 \\
53 & 45 & 07\end{array}$ & $\begin{array}{ccc}\circ & \prime & \prime \prime \\
189 & 45 & 28 \\
199 & 09 & 45 \\
233 & 41 & 09\end{array}$ & $\begin{array}{r}\text { Log. meters. } \\
4.19534 \\
4.48140 \\
4.08812\end{array}$ & $\begin{array}{r}\text { Miles. } \\
9.743 \\
18.827 \\
7.611\end{array}$ \\
\hline
\end{tabular}

\section{INDIAN, FRESNO COUNTY, CALIF.}

On a bald mountain at the head of Indian Valley and on the north side of Panoche Creek. It is about 7 miles northeast by east of Panoche post office. The PanocheMendota road intersects the road from Idria 4 miles south of the Kern oil well. From the Kern oil well the signal can be seen, probably 5 miles distant. Animals can be ridden up almost any of the ridges leading to the highest point.

Signal: Cairn 7 feet high. 
Station mark: Triangulation tablet set in rock; distant 1.60 meters from signal, azimuth $41^{\circ}$ to signal.

See Bulletin $440 \mathrm{~N}$, pp. 69 and 90.

[Latitude $36^{\circ} 38^{\prime} 27.298^{\prime \prime}$. Longitude $120^{\circ} 43^{\prime} 34.341^{\prime \prime}$.]

\begin{tabular}{|c|c|c|c|c|}
\hline Tostation- & Azimuth. & Back azimuth. & \multicolumn{2}{|c|}{ Distance. } \\
\hline 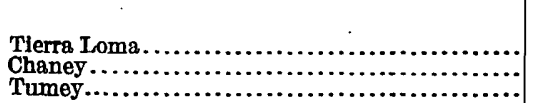 & $\begin{array}{ccc}\circ & \prime & \\
212 & 23 & 54 \\
263 & 42 & 39 \\
332 & 32 & 36\end{array}$ & $\begin{array}{rcc}\circ & \prime \prime & \\
32 & 26 & 22 \\
83 & 48 & 01 \\
152 & 35 & 02\end{array}$ & \begin{tabular}{|r|} 
Log. meters. \\
4.05886 \\
4.12871 \\
4.11989
\end{tabular} & $\begin{array}{r}\text { Miles. } \\
7.116 \\
8.357 \\
8.189\end{array}$ \\
\hline
\end{tabular}

LAGUNA SECA FIAG, MERCED COUNTY, CALIF. (Not occuplod.)

On highest point of hill, 1 mile east of Laguna Seca ranch house.

Station mark: Small cairn with 2 by 2 redwood flagpole.

[Latitude $36^{\circ} 52^{\prime} 02.025^{\prime \prime}$. Longitude $120^{\circ} 50^{\prime} 43.82^{\prime \prime}$.]

\begin{tabular}{|c|c|c|c|c|}
\hline Tostation- & Azimuth. & Back azimuth. & \multicolumn{2}{|c|}{ Distance. } \\
\hline $\begin{array}{l}\text { Bonanza } \\
\text { Wright. } \\
\text { White......... }\end{array}$ & \begin{tabular}{rcc}
\multicolumn{1}{c}{} & $\prime$ & $\prime \prime$ \\
60 & 36 & 04.37 \\
129 & 58 & 57.90 \\
184 & 33 & 30.64
\end{tabular} & \begin{tabular}{rcc}
\multicolumn{1}{c}{} & \multicolumn{1}{c}{} & $\prime \prime$ \\
240 & 31 & 13.02 \\
309 & 53 & 57.02 \\
4 & 33 & 40.56
\end{tabular} & \begin{tabular}{|r|} 
Log. meters. \\
4.14073 \\
4.20882 \\
3.71147
\end{tabular} & $\begin{array}{r}\text { Miles. } \\
8.592 \\
10.050 \\
3.198\end{array}$ \\
\hline
\end{tabular}

LANGAN, MERCED COUNTY, CAIIF.

On the highest point of hill, 2 miles southeast of F. E. Langan's ranch house and about 16 miles south of Los Banos.

Signal: A lumber structure with cross targets.

Station mark: A bronze triangulation tablet in rock flush with ground under eignal.

[Latitude $36^{\circ} 50^{\prime} 30.38^{\prime \prime}$. Longitude $120^{\circ} 53^{\prime} 07.21^{\prime \prime}$.]

\begin{tabular}{|c|c|c|c|c|}
\hline Tostation- & Azimuth. & Back azimuth. & Dist8. & ce. \\
\hline 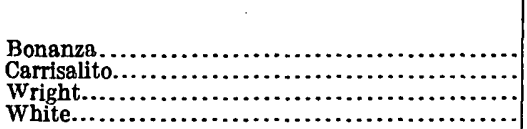 & $\begin{array}{rcc}\circ & \prime & \prime \prime \\
64 & 57 & 44.68 \\
102 & 20 & 38.38 \\
146 & 11 & 57.92 \\
206 & 27 & 11.98\end{array}$ & \begin{tabular}{rcc|}
\multicolumn{1}{c}{,} & $\prime$ & $\prime \prime$ \\
244 & 54 & 19.36 \\
282 & 15 & 24.00 \\
326 & 08 & 23.23 \\
26 & 28 & 47.95
\end{tabular} & $\begin{array}{r}\text { Log. meter } 8 . \\
3.9718627 \\
4.1235201 \\
4.2014256 \\
3.9487170\end{array}$ & $\begin{array}{r}\text { Miles. } \\
5.824 \\
8.258 \\
9.880 \\
5.522\end{array}$ \\
\hline
\end{tabular}

LEVIS, FRESNO COUNTY, CAIIF. (Not oocupled.)

Station is a smokestack at Levis pump station, 8 miles south of Mendota.

Station mark: Center of stack.

[Latitude $36^{\circ} 37^{\prime} 23.95^{\prime \prime}$. Longitude $120^{\circ} 24^{\prime} 22.67^{\prime \prime}$.]

\begin{tabular}{|c|c|c|c|c|}
\hline Tostation- & Azimuth. & Back azimuth. & Distay & $\infty$. \\
\hline 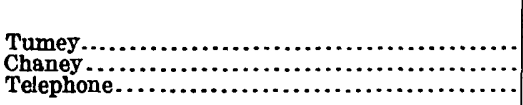 & $\begin{array}{rcc}\circ & \prime & \prime \prime \\
66 & 42 & 22 \\
102 & 41 & 03 \\
128 & 15 & 04\end{array}$ & $\begin{array}{ccc}\circ & \prime & \prime \prime \\
246 & 34 & 16 \\
282 & 34 & 57 \\
308 & 11 & 11\end{array}$ & $\begin{array}{r}\text { Log. meters. } \\
4.39042 \\
4.19361 \\
4.09021\end{array}$ & $\begin{array}{r}\text { Miles. } \\
15.268 \\
9.704 \\
7.648\end{array}$ \\
\hline
\end{tabular}

TELEPHONE, FRESNO COUNTY, CALIF.

The windmill derrick at the Miller \& Lux Co.'s sheep camp, known as the Telephone ranch, 10 miles from Mendota, a town on the Southern Pacific R. R., and 0.5 mile north of the Idria-Mendota road. The Chaney ranch is 4 miles west on the same 
road and is the only ranch of importance between Mendota and Idria. Hay and stock water can be had at the Telephone ranch but no water fit to drink.

Station mark: The pipe extending into ground.

[Latitude $36^{\circ} 41^{\prime} 30.991^{\prime \prime}$. Longitude $120^{\circ} 30^{\prime} 52.018^{\prime \prime}$.]

\begin{tabular}{|c|c|c|c|c|}
\hline Tostation- & Azimuth. & Back azimuth. & \multicolumn{2}{|c|}{ Distance. } \\
\hline 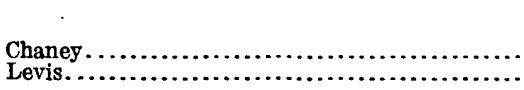 & $\begin{array}{rcc}\circ & \prime & \prime \prime \\
52 & 58 & 34 \\
308 & 11 & 11\end{array}$ & $\begin{array}{ccc}\circ & \prime & \prime \prime \\
232 & 56 & 21 \\
128 & 15 & 04\end{array}$ & $\begin{array}{r}\text { Log. meters. } \\
3.04320 \\
4.09021\end{array}$ & $\begin{array}{r}\text { Miles. } \\
\quad 4.532 \\
\quad 7.648\end{array}$ \\
\hline
\end{tabular}

TIERRA LOMA, FRESHO COUNTY, CALIF.

Station is windmill at the Tierra Loma Schoolhouse 20 miles south of Dos Palos.

Signal: Center of mill.

Station mark: Center of well.

[Latitude $36^{\circ} 43^{\prime} 40.905^{\prime \prime}$. Longitude $120^{\circ} 39^{\prime} 27.069^{\prime \prime}$.]

\begin{tabular}{|c|c|c|c|c|}
\hline Tostation- & Azimuth. & Back azimuth. & DistaI & ce. \\
\hline 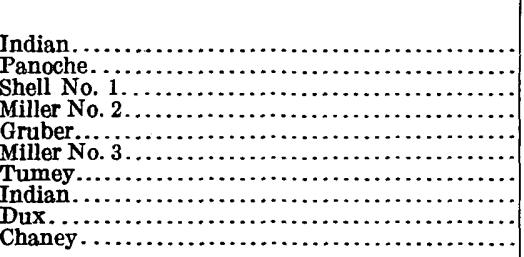 & $\begin{array}{rll}\circ & \prime & \prime \prime \\
32 & 26 & 21.73 \\
88 & 35 & 06.15 \\
153 & 33 & 33.21 \\
184 & 50 & 59.61 \\
211 & 43 & 38.63 \\
249 & 43 & 47.76 \\
00 & 11 & 59 \\
32 & 26 & 22 \\
233 & 41 & 09 \\
318 & 36 & 48\end{array}$ & \begin{tabular}{rcc}
\multicolumn{1}{c}{} & \multicolumn{1}{c}{$\prime \prime$} \\
212 & 23 & 54.00 \\
269 & 31 & 17.18 \\
333 & 32 & 32.07 \\
4 & 41 & 24.67 \\
31 & 44 & 36.66 \\
69 & 47 & 18.14 \\
180 & 11 & 57 \\
212 & 23 & 54 \\
53 & 45 & 07 \\
138 & 39 & 46
\end{tabular} & $\begin{array}{l}\text { Log. meters. } \\
\text { 4. } 0588652 \\
\text { 3. } 9779082 \\
3.7552258 \\
\text { 4. } 1038132 \\
\text { 3. } 6604077 \\
\text { 3. } 9684437 \\
4.32968 \\
4.05886 \\
4.08812 \\
4.03864\end{array}$ & $\begin{array}{r}\text { Miles. } \\
7.116 \\
5.906 \\
3.537 \\
7.892 \\
2.843 \\
5.778 \\
13.275 \\
7.116 \\
7.611 \\
6.792\end{array}$ \\
\hline
\end{tabular}

TUMEY, FRESNO COUNTY, CALIF.

On a high foothill 5 miles south of mouth of Panoche Canyon. From Mendota go 20 miles southwestward to mouth of Panoche Canyon; continue up canyon 5 miles to forks of New Idria and Panoche roads, thence southeastward along trail up west side of canyon. Trail passes just east of station.

Signal: A 2 by 2 inch redwood pole bearing a flag.

Station mark: A sandstone boulder, in which is a 1-inch drill hole, at base of flag.

Latitude $36^{\circ} 32^{\prime} 07.82^{\prime \prime}$. Longitude $120^{\circ} 39^{\prime} 20.07^{\prime \prime}$.]

\begin{tabular}{|c|c|c|c|c|}
\hline Tostation- & Azimuth. & Back azimuth. & Dista & ce. \\
\hline 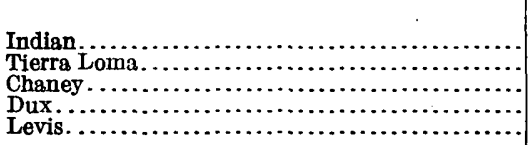 & $\begin{array}{ccc}\circ & \prime & \prime \prime \\
152 & 35 & 02 \\
180 & 11 & 57 \\
209 & 00 & 54 \\
199 & 09 & 45 \\
246 & 34 & 16\end{array}$ & \begin{tabular}{rll}
\multicolumn{1}{c}{} & $\prime$ & $\prime \prime$ \\
332 & 32 & 36 \\
00 & 11 & 59 \\
29 & 03 & 53 \\
19 & 13 & 44 \\
66 & 42 & 22
\end{tabular} & \begin{tabular}{r|} 
Log. meters. \\
4.11989 \\
4.32968 \\
4.17760 \\
4.48140 \\
4.39042
\end{tabular} & $\begin{array}{r}\text { Miles. } \\
8.189 \\
13.275 \\
9.357 \\
18.827 \\
15.268\end{array}$ \\
\hline
\end{tabular}

VALLEY PIPE LINE NO. 5, MERCED COUNTY, CALIF.

Station and signal: Center of smokestack at Valley Pump Line Co.'s station about 8 miles south of Los Banos.

[Latitude $36^{\circ} 58^{\prime} 16.73^{\prime \prime}$. Longitude $120^{\circ} 52^{\prime} 55.90^{\prime \prime}$.]

\begin{tabular}{|c|c|c|c|c|}
\hline To station- & Azimuth. & Back azimuth. & Distar & \\
\hline 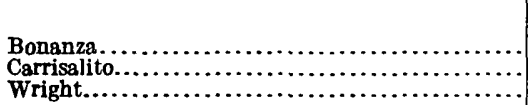 & $\begin{array}{ccc}\circ & \prime & \prime \prime \\
25 & 33 & 36 \\
48 & 59 & 15 \\
82 & 44 & 37\end{array}$ & $\begin{array}{ccc}\circ & \prime & \prime \prime \\
205 & 30 & 04 \\
228 & 53 & 55 \\
262 & 40 & 56\end{array}$ & $\begin{array}{r}\text { Log. meters. } \\
4.30818 \\
4.24494 \\
3.96376\end{array}$ & $\begin{array}{r}\text { Miles. } \\
12.634 \\
10.922 \\
5.716\end{array}$ \\
\hline
\end{tabular}


WHITE, MERCED COUNTY, CAITF.

On a bald plateau about 4 miles southwest of the Miller \& Lux Co.'s Jamison ranch, which is on the westernmost canal and 9 miles south of Los Banos. It is about 11 miles by road west of South Dos Palos. The signal is on a smooth, grass-covered hill with no rock on it. There is a point a little higher about 0.25 mile south. Animals can be ridden to station; a light wagon even may be driven to it, though the hill is rather steep.

Signal: A 4 by 4 inch pole, standing 12 feet above ground, on which are two targets at right angles to each other.

Station mark: Triangulation tablet in rock placed in ground 0.65 meter northwest of signal. The rock is 6 inches above ground on the northwest side of iablet.

[Latitude $36^{\circ} 54^{\prime} 48.435^{\prime \prime}$. Longitude $\left.120^{\circ} 50^{\prime} 27.300 . .^{\prime \prime}\right]$

\begin{tabular}{|c|c|c|c|c|}
\hline To station- & Azimuth. & Back azimuth. & Dista & \\
\hline 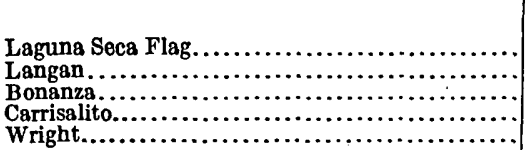 & \begin{tabular}{rcc}
$\circ$ & \multicolumn{1}{r}{} & \multicolumn{1}{c}{ ' } \\
4 & 33 & 40.56 \\
26 & 28 & 47.95 \\
46 & 16 & 03.78 \\
73 & 13 & 37.67 \\
112 & 21 & 01.33
\end{tabular} & $\begin{array}{ccc}\circ & \prime & \prime \prime \\
184 & 33 & 30.64 \\
206 & 27 & 11.98 \\
225 & 11 & 02.36 \\
253 & 06 & 47.04 \\
292 & 15 & 50.36\end{array}$ & $\begin{array}{c}\text { Log. meters. } \\
\text { 3.71147 } \\
3.9487170 \\
4.2364938 \\
4.2478847 \\
4.1411907\end{array}$ & $\begin{array}{r}\text { Miles. } \\
3.198 \\
5.522 \\
10.711 \\
10.996 \\
8.601\end{array}$ \\
\hline
\end{tabular}

WRIGHT, MERCED COUNTY, CALIF.

On highest point of hill, 1 mile south of Los Banos Creek and 2 miles northeast of Gaston Bede ranch house.

Station mark: Cross on rock flush with ground under small cairn.

Signal: A timber structure on which are cross targets.

Reference mark: A bronze tablet 24.6 feet from signal, azimuth $64^{\circ} 08^{\prime}$.

[Latitude $36^{\circ} 57^{\prime} 35.87^{\prime \prime}$. Longitude $120^{\circ} 59^{\prime} 04.78^{\prime \prime}$.]

\begin{tabular}{|c|c|c|c|c|}
\hline To station- & Arimuth. & Back azimuth. & Distar & \\
\hline 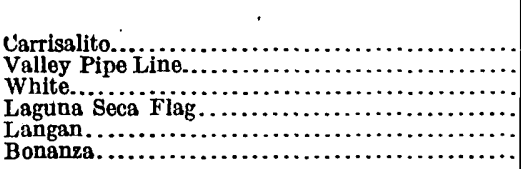 & $\begin{array}{rcc}\circ & \prime & \prime \prime \\
21 & 41 & 02.51 \\
262 & 40 & 56 \\
292 & 15 & 50.36 \\
309 & 53 & 57.02 \\
326 & 08 & 23.23 \\
358 & 45 & 37.10\end{array}$ & $\begin{array}{rcc}\circ & \prime & \prime \prime \\
201 & 39 & 22.46 \\
82 & 44 & 37 \\
112 & 21 & 01.33 \\
129 & 58 & 57.90 \\
146 & 11 & 57.92 \\
178 & 45 & 46.10\end{array}$ & $\begin{array}{c}\text { Log. meters. } \\
4.0479295 \\
3.96376 \\
4.1411907 \\
4.20882 \\
4.2014256 \\
4.2351166\end{array}$ & $\begin{array}{r}\text { Miles. } \\
6.939 \\
5.716 \\
8.601 \\
10.050 \\
9.880 \\
10.677\end{array}$ \\
\hline
\end{tabular}

Avenal, Lapanza, Nipomo, and Pozo quadrangles.

KERN, SAN LUIS OBISPO, AND SANTA BARBARA COUNTIES.

In the spring of $1919 \mathrm{~L}$. F. Biggs extended triangulation over the above-named quadrangles, based on stations San Luis and San Jose of the U. S. Coast and Geodetic Survey.

Fifteen stations were located on North American datum.

ALIEN, SAT LUIS OBISPO COUNTY, CALIF.

On north end and highest point of long timbered ridge, just west of the Carrizo ranch.

From Simmler follow road past Carrizo ranch to Buckhorn ranch, thence go southwest by trail up canyon to summit, and continue southwestward to second summit where point can be seen and approached to the southward. Point can also be reached from Avenal ranch.

Station mark: Bronze tablet in rock under signal.

Signal: Tree and small cairn over tablet.

$121166^{\circ}-23-$ Bull. $709-38$ 
[Latitude $35^{\circ} 11^{\prime} 07.97^{\prime \prime}$. Longitude $120^{\circ} 05^{\prime} 03.79^{\prime \prime}$.]

\begin{tabular}{|c|c|c|c|c|}
\hline To station- & Azimuth. & Back azimuth. & Distan & e. \\
\hline 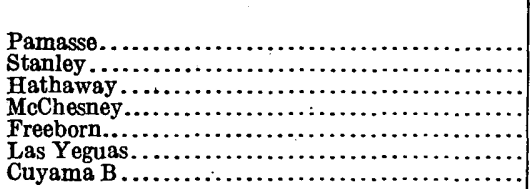 & \begin{tabular}{rcc}
\multicolumn{1}{c}{} & \multicolumn{1}{c}{ ' } \\
24 & 27 & 57.53 \\
50 & 37 & 39.48 \\
88 & 39 & 52.24 \\
128 & 01 & 38.17 \\
194 & 10 & 10.82 \\
202 & 17 & 37.99 \\
356 & 48 & 32.95
\end{tabular} & \begin{tabular}{rcc}
\multicolumn{1}{c}{0} & $\prime$ & $\prime \prime$ \\
204 & 25 & 36.53 \\
230 & 31 & 55.01 \\
268 & 34 & 39.45 \\
307 & 56 & 34.97 \\
14 & 11 & 14.80 \\
22 & 22 & 04.74 \\
176 & 48 & 56.78
\end{tabular} & \begin{tabular}{r|} 
Log. meters. \\
4. 1759076 \\
4. 2926926 \\
4.1379504 \\
4.2269035 \\
4.0587497 \\
4.4868705 \\
4.2759012
\end{tabular} & $\begin{array}{r}\text { Miles. } \\
9.317 \\
12.191 \\
8.537 \\
10.477 \\
7.114 \\
19.065 \\
11.729\end{array}$ \\
\hline
\end{tabular}

ChICHES, SAN IUIS OBISPO COUNTY, CALIF.

On a high brushy peak, 9 miles west of Pozo. From Pozo follow Simmler road 7.0 miles to top of Pozo grade and take road to left 1 mile to Ranger Cabin. A trail leads from Ranger Cabin to station; distance about 4 miles.

Station and signal: A 10 -foot tree placed in the ground.

Reference mark: A bronze tablet in rock flush with the ground, distant 1.5 feet from station; azimuth from tablet to station $236^{\circ}$.

[Latitude $35^{\circ} 23^{\prime} 34.30^{\prime \prime}$. Longitude $120^{\circ} 21^{\prime} 05.095^{\prime \prime}$.]

\begin{tabular}{|c|c|c|c|c|}
\hline$\cdots$ Tostation- & Azimuth. & Back azimuth. & Distar & ce. \\
\hline 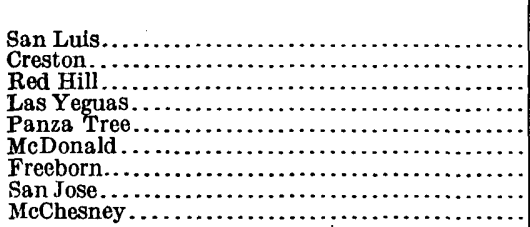 & \begin{tabular}{rcc}
\multicolumn{1}{c}{} & $\prime$ & $\prime \prime$ \\
56 & 19 & 25.42 \\
150 & 57 & 55.20 \\
200 & 57 & 41.63 \\
261 & 21 & 39.98 \\
279 & 05 & 26.31 \\
279 & 11 & 19.99 \\
293 & 38 & 20.85 \\
318 & 51 & 34.49 \\
318 & 52 & 59.07
\end{tabular} & \begin{tabular}{rcc}
\multicolumn{1}{c}{} & $\prime$ & $\prime \prime$ \\
236 & 12 & 08.76 \\
330 & 54 & 03.74 \\
21 & 01 & 05.47 \\
81 & 35 & 24.51 \\
99 & 08 & 49.58 \\
99 & 20 & 16.03 \\
113 & 48 & 41.03 \\
138 & 54 & 26.26 \\
138 & 57 & 11.08
\end{tabular} & \begin{tabular}{r|} 
Log. meters. \\
4. 3603682 \\
4.3158950 \\
4.3927193 \\
4.5598618 \\
3.9529260 \\
4.3743874 \\
4.4709454 \\
4.0568484 \\
4.2238956
\end{tabular} & $\begin{array}{r}\text { Miles. } \\
14.280 \\
13.160 \\
15.348 \\
22.553 \\
5.575 \\
14.714 \\
18.378 \\
7.083 \\
10.405\end{array}$ \\
\hline
\end{tabular}

CRESTON, SAN LUIS OBISPO COUNTY, CALIF.

On fence line half a mile south of road, on highest point of bare hill, 6 miles east of Creston. From Creston take Shandon road 5 miles to foot of hill, here take right-hand fork and follow past summit one-quarter of a mile westward.

Station mark: A bronże tablet in rock flush with ground under fence and signal.

Signal: A timber structure, on which are cross targets.

[Latitude $35^{\circ} 33^{\prime} 21.28^{\prime \prime}$. Longitude $120^{\circ} 27^{\prime} 43.92^{\prime \prime}$.]

\begin{tabular}{|c|c|c|c|c|}
\hline To station- & Azimuth. & Back azimuth. & Distar & \\
\hline 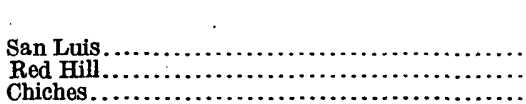 & $\begin{array}{ccc}\circ & \prime & \prime \prime \\
16 & 16 & 52.18 \\
255 & 11 & 07.20 \\
330 & 54 & 03.74\end{array}$ & \begin{tabular}{rcc}
\multicolumn{1}{c}{} & $\prime$ & $\prime \prime$ \\
196 & 13 & 25.78 \\
75 & 28 & 23.49 \\
150 & 57 & 55.20
\end{tabular} & \begin{tabular}{|} 
Log. meters. \\
4. 5065952 \\
4.2905877 \\
4.3158950
\end{tabular} & $\begin{array}{r}\text { Miles. } \\
19.996 \\
12.132 \\
13.160\end{array}$ \\
\hline
\end{tabular}

CUYAMA B, SANTA BARBARA COUNTY, CALIF. (Not occupied.)

On a sharp brushy ridge, about 4 miles south of Pamasse ranch house, which is in the Santa Maria Canyon about 25 miles by road east of Santa Maria.

Station and signal: Highest point of hill.

[Latitude $35^{\circ} 00^{\prime} 56.40^{\prime \prime}$. Longitude $120^{\circ} 04^{\prime} 22.35^{\prime \prime}$.]

\begin{tabular}{|c|c|c|c|c|c|}
\hline 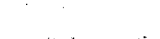 & To station- & Azimuth. & Back azimuth. & Dista & \\
\hline $\begin{array}{l}\text { McChesney... } \\
\text { Allen........ } \\
\text { Las Yeguas.. }\end{array}$ & ............. & $\begin{array}{ccc}\circ & \prime & \prime \prime \\
153 & 53 & 18.25 \\
176 & 48 & 56.78 \\
192 & 38 & 30.52\end{array}$ & \begin{tabular}{rcc}
\multicolumn{1}{c}{$\circ$} & $\prime$ & $\prime \prime$ \\
333 & 47 & 51.83 \\
356 & 48 & 32.95 \\
12 & 42 & 32.81
\end{tabular} & \begin{tabular}{|r|} 
Log. meters. \\
4.5126632 \\
4.2759012 \\
4.6849016
\end{tabular} & $\begin{array}{r}\text { Miles. } \\
20.231 \\
11.729 \\
30.078\end{array}$ \\
\hline
\end{tabular}


FREEBORN, SAN LUIS OBISPO COUNTY, CAIIF.

On highest point of hill, 6 miles southwest of Simmler. From Cavanaugh \& Freeborn ranch skirt hill 1 mile eastward to canyon; follow trail up canyon to pass, thence along ridge to summit. Station can also be approached from south.

Station mark: A bronze tablet set in large rock under signal.

Signal: A 12-foot tree braced to boulder.

[Latitude $35^{\circ} 17^{\prime} 08.15^{\prime \prime}$. Longitude $120^{\circ} 03^{\prime} 12.89^{\prime \prime}$.]

\begin{tabular}{|c|c|c|c|c|}
\hline Tostation- & Azimuth. & Back azimuth. & Dista & \\
\hline 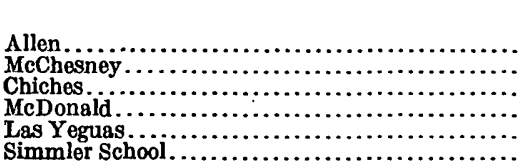 & $\begin{array}{rcc}\circ & \prime & \prime \prime \\
14 & 11 & 14.80 \\
87 & 28 & 50.41 \\
113 & 48 & 41.03 \\
155 & 27 & 21.51 \\
207 & 05 & 30.42 \\
216 & 02 & 56.42\end{array}$ & \begin{tabular}{rcc}
\multicolumn{1}{c}{} & $\prime$ & $\prime \prime$ \\
194 & 10 & 10.82 \\
267 & 22 & 42.78 \\
293 & 38 & 20.85 \\
335 & 25 & 56.92 \\
27 & 08 & 53.32 \\
36 & 05 & 58.32
\end{tabular} & \begin{tabular}{|r|} 
Log. meters. \\
4.0587497 \\
4.2068429 \\
4.4709454 \\
3.9490249 \\
4.2881364 \\
3.9565127
\end{tabular} & $\begin{array}{r}\text { Miles. } \\
7.114 \\
10.004 \\
18.374 \\
5.526 \\
12.064 \\
5.622\end{array}$ \\
\hline
\end{tabular}

HATHAWAY, SAN LUIS OBISPO COUNTY, CALIF.

On brush -covered peak, 1 mile south of Pine Mountain and 2 miles north of Hathaway ranch house. From Huasna take road eastward to top of hill three-quarters of a mile east of William Lane's ranch house and half a mile west of Hathr.way ranch house. Take left-hand road up hill; continue around hill 11 miles to east slope of hill, on which are scattering oaks. Station is half a mile west of road.

Station mark: Cross on rock buried flush with ground under signal.

Signal: Live-oak tree 10 feet high, set in ground.

[Latitude $35^{\circ} 10^{\prime} 57.25^{\prime \prime}$. Longitude $120^{\circ} 14^{\prime}$ 06.64.']

\begin{tabular}{|c|c|c|c|c|}
\hline Tostation- & Azimuth. & Back azimuth. & Dista & \\
\hline 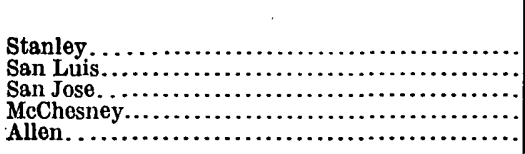 & $\begin{array}{rcc}\circ & \prime \prime \\
6 & 38 & 44.53 \\
109 & 44 & 47.56 \\
168 & 13 & 58.95 \\
182 & 20 & 04.08 \\
268 & 34 & 39.45\end{array}$ & \begin{tabular}{rcc}
\multicolumn{1}{c}{} & $\prime$ & $\prime \prime$ \\
186 & 38 & 16.43 \\
289 & 33 & 29.91 \\
348 & 12 & 48.04 \\
2 & 20 & 14.04 \\
88 & 39 & 52.24
\end{tabular} & \begin{tabular}{|r|} 
Log. meters. \\
4.0867225 \\
4.4984270 \\
4.1778311 \\
4.0300830 \\
4.1379504
\end{tabular} & $\begin{array}{r}\text { Miles. } \\
7.587 \\
19.578 \\
9.358 \\
6.659 \\
8.537\end{array}$ \\
\hline
\end{tabular}

IOMA PAIOMA, SAN LUIS OBISPO COUNTY, CAIIF. (Not occupled.)

Station is a small leaning tree on small peak in saddle on high bare ridge northeast of Arroyo Grande.

Station mark and signal: $\Lambda$ tree.

[Latitude $35^{\circ} 09^{\prime} 54.71^{\prime \prime}$. Longitude $120^{\circ} 28^{\prime} 35.63^{\prime \prime}$.]

\begin{tabular}{|c|c|c|c|c|}
\hline Tostation- & Azimuth. & Back azimuth. & Distar & ce. \\
\hline 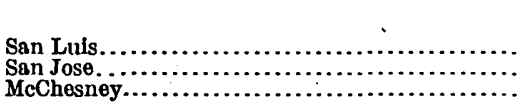 & $\begin{array}{ccc}\circ & \prime & \prime \prime \\
\cdot 148 & 26 & 29.31 \\
228 & 31 & 23.75 \\
240 & 31 & 01.71\end{array}$ & \begin{tabular}{rcc}
\multicolumn{1}{c}{$\circ$} & $\prime \prime$ & $\prime \prime$ \\
328 & 23 & 33.72 \\
48 & 38 & 35.01 \\
60 & 39 & 32.88
\end{tabular} & \begin{tabular}{|r|} 
Log. meters. \\
4.1673568 \\
4.4013924 \\
4.4104343
\end{tabular} & $\begin{array}{r}\text { Miles. } \\
9.135 \\
15.658 \\
15.988\end{array}$ \\
\hline
\end{tabular}

\section{LAS YEGUAS, KERN COUNTY, CALIF.}

On highest point of hill 7 miles north of Simmler and $1 \frac{1}{2}$ miles southwest of Las Yeguas ranch house; road passes through saddle one-eighth of a mile north of station.

Station mark: Tablet set in rock flush with ground under signal.

Signal: A timber structure on which are cross targets. 
[Latitude $35^{\circ} 26^{\prime} 28.88^{\prime \prime}$. Longitude $119^{\circ} 57^{\prime} 22.32^{\prime \prime}$.]

\begin{tabular}{|c|c|c|c|c|}
\hline Tostation- & Azimuth. & Back azimuth. & Dista & ce. \\
\hline 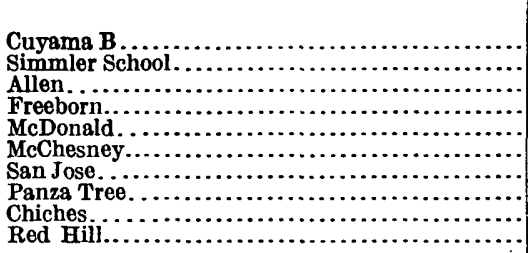 & $\begin{array}{rcc}\circ & \prime \prime & \prime \prime \\
12 & 42 & 32.81 \\
19 & 29 & 29.53 \\
22 & 22 & 04.74 \\
27 & 08 & 53.32 \\
53 & 47 & 39.41 \\
54 & 14 & 01.13 \\
63 & 55 & 01.86 \\
75 & 57 & 59.52 \\
81 & 35 & 24.51 \\
123 & 18 & 10.95\end{array}$ & $\begin{array}{ccc}\circ & \prime & \prime \prime \\
192 & 38 & 30.52 \\
199 & 28 & 08.60 \\
202 & 17 & 37.99 \\
207 & 05 & 30.42 \\
233 & 42 & 51.58 \\
234 & 04 & 32.91 \\
243 & 44 & 09.98 \\
255 & 47 & 38.52 \\
261 & 21 & 39.98 \\
303 & 07 & 48.30\end{array}$ & $\begin{array}{r}\text { Log. meters. } \\
4.6849016 \\
4.0241725 \\
4.4868705 \\
4.2851364 \\
4.1916443 \\
4.48773,6 \\
4.5005937 \\
4.4453531 \\
4.5598618 \\
4.5089033\end{array}$ & $\begin{array}{r}\text { Miles. } \\
30.078 \\
6.569 \\
19.065 \\
12.064 \\
9.660 \\
19.102 \\
19.676 \\
17.366 \\
22.553 \\
20.056\end{array}$ \\
\hline
\end{tabular}

M'CAESTEY, SAN LUIS OBISPO COUNTY, CALIF.

Near U. S. Coast and Geodetic Survey point "Dome."

On a high brushy dome-shaped mountain, 10 miles southeast of Pozo. From Pozo follow the Avenal road 10 miles to Conway's ranch. From Conways follow trait up canyon to eastward, keeping just south of east-west fence; follow this fence about 2 miles to foot of point leading to brushy ridge on which are scattering pine trees; follow trail past pines to top of hill and signal, 4 miles from Conways.

Station mark: A bronze tablet set in large boulder under signal.

Signal: A 12-foot tree braced over tablet.

[Latitude $35^{\circ} 16^{\prime} 44.723^{\prime \prime}$. Longitude $120^{\circ} 13^{\prime} 49.362^{\prime \prime}$.]

\begin{tabular}{|c|c|c|c|c|}
\hline Tostation- & Azimuth. & Back azimuth. & Dista & \\
\hline 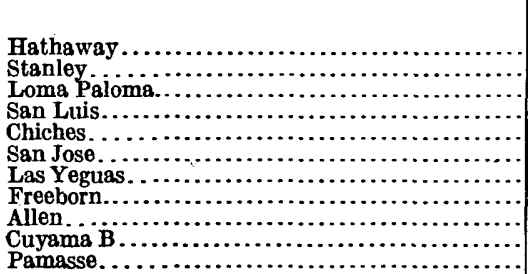 & $\begin{array}{rcc}\circ & \prime \prime \\
2 & 20 & 14.04 \\
4 & 38 & 02.21 \\
60 & 39 & 32.88 \\
89 & 52 & 58.66 \\
138 & 57 & 11.88 \\
139 & 00 & 11.44 \\
231 & 04 & 32.91 \\
267 & 22 & 42.78 \\
307 & 56 & 34.97 \\
333 & 47 & 51.83 \\
313 & 31 & 06.36\end{array}$ & \begin{tabular}{rcc}
\multicolumn{1}{c}{} & \multicolumn{1}{c}{} & \multicolumn{1}{c}{} \\
182 & 20 & 04.08 \\
184 & 37 & 20.12 \\
210 & 31 & 01.71 \\
269 & 41 & 30.95 \\
318 & 52 & 59.07 \\
318 & 58 & 51.20 \\
54 & 14 & 04.13 \\
87 & 28 & 50.41 \\
128 & 01 & 38.17 \\
153 & 53 & 18.25 \\
163 & 33 & 47.94
\end{tabular} & $\begin{array}{r}\text { Log. meters. } \\
\text { 4. } 0300830 \\
4.3600442 \\
\text { 4. } 4104343 \\
4.4784551 \\
4.2238956 \\
3.7281113 \\
4.4877346 \\
4.2068429 \\
4.2269035 \\
4.5126632 \\
4.3988740\end{array}$ & $\begin{array}{r}\text { Miles. } \\
6.659 \\
14.236 \\
15.988 \\
18.698 \\
10.405 \\
3.322 \\
19.102 \\
10.004 \\
10.477 \\
20.231 \\
15.568\end{array}$ \\
\hline
\end{tabular}

M'DONALD, SAN LUIS OBISPO COUNTY, CALIF.

On the south end and at highest point of mesa, 1 mile south of Pozo-Simmler road and 6 miles west of Simmler.

Station mark: Tablet cemented in rock under signal.

Signal: A 10-foot cedar tree braced over station.

[Latitude $35^{\circ} 21^{\prime} 30.61^{\prime \prime}$. Longitude $120^{\circ} 05^{\prime} 39.20^{\prime \prime}$.]

\begin{tabular}{|c|c|c|c|c|}
\hline Tostation- & Azimuth. & Back azimuth. & Distar & ce. \\
\hline 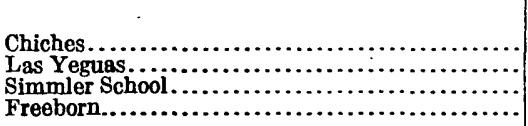 & $\begin{array}{rcc}\circ & \prime & \prime \prime \\
99 & 20 & 16.03 \\
233 & 42 & 51.58 \\
274 & 53 & 01.24 \\
335 & 25 & 56.92\end{array}$ & \begin{tabular}{rcc}
\multicolumn{1}{c}{} & $\prime$ & $\prime \prime$ \\
279 & 11 & 19.99 \\
53 & 47 & 39.41 \\
94 & 56 & 27.91 \\
155 & 27 & 21.51
\end{tabular} & \begin{tabular}{|r|} 
Log. meters. \\
4. 3743874 \\
4. 1916443 \\
3. 9567018 \\
3. 9490249
\end{tabular} & $\begin{array}{r}\text { Miles. } \\
14.714 \\
9.660 \\
5.624 \\
5.526\end{array}$ \\
\hline
\end{tabular}

PAMASSE, SATTA BARBara COUTTY, CALIF.

On a small butte, elevation about 2,500 feet, 1 mile south of Pamasse ranch house, on Santa Maria River, about 25 miles by road from Santa Maria. The ridge increaseis in height toward the southeast. From Santa Maria follow the Santa Maria River road to point 3 miles beyond where road leaves river to pass the Narrows, to gate with signboard reading "Santa Barbara National Forest Boundary"; continue one-eighth mile farther to small saddle in which is an iron post marked "A. T. Co. Elev. 1388. Sta. $2234 \pm 52$ ", thence go up open ridge 1 mile to signal. 
Station mark: Aluminum tablet set in rock on small butte.

Signal is a double oak tree bearing a white cloth, 19 feet distant, azimuth $320^{\circ}$ from station.

[Latitude $35^{\circ} 03^{\circ} 45.05^{\prime \prime}$. Longitude $120^{\circ} 09^{\prime} 08.85^{\prime \prime}$.]

\begin{tabular}{|c|c|c|c|c|}
\hline To station- & Azimtath. & Back azimuth. & Dista & ce. \\
\hline 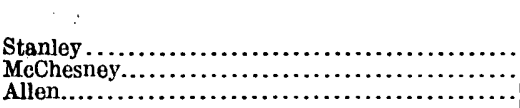 & $\begin{array}{rcc}\circ & \prime & \prime \prime \\
97 & 36 & 00.76 \\
163 & 33 & 47.94 \\
204 & 25 & 36.53\end{array}$ & \begin{tabular}{rcc}
\multicolumn{1}{c}{} & $\prime$ & $\prime \prime$ \\
277 & 32 & 37.62 \\
343 & 31 & 06.36 \\
24 & 27 & 57.53
\end{tabular} & \begin{tabular}{|r|} 
Log. meters. \\
3.9560414 \\
4.3988740 \\
4.1759076
\end{tabular} & $\begin{array}{r}\text { Miles. } \\
5.616 \\
15.568 \\
9.317\end{array}$ \\
\hline
\end{tabular}

PANZA TREE, SAN LUIS OBISPO COUNTY, CALIF. (Not occupled.)

Station is a pine tree on top of hill 4 miles north of La Panza.

Signal and station mark is the tree.

[Latitude $35^{\circ} 22^{\prime} 48.16^{\prime \prime}$. Longitude $120^{\circ} 15^{\prime} 14.07^{\prime \prime}$.]

\begin{tabular}{|c|c|c|c|c|}
\hline Tostation- & Azimuth. & Back azimuth. & Dista & \\
\hline 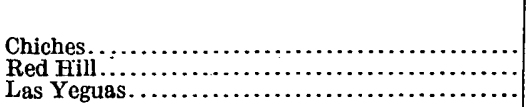 & $\begin{array}{rcc}\circ & \prime & \prime \prime \\
99 & 08 & 49.58 \\
180 & 00 & 06.62 \\
255 & 47 & 38.52\end{array}$ & \begin{tabular}{rcc|}
\multicolumn{1}{c}{$c$} & $\prime$ & $\prime \prime$ \\
279 & 05 & 26.31 \\
00 & 00 & 06.63 \\
75 & 57 & 59.52
\end{tabular} & \begin{tabular}{|r|} 
Log. meters. \\
3. 9529260 \\
4. 3888864 \\
4.4453531
\end{tabular} & $\begin{array}{r}\text { Miles. } \\
5.575 \\
15.214 \\
17.366\end{array}$ \\
\hline
\end{tabular}

RED HILL, SAN LUIS OBISPO COUNTY, CALIF.

On south end of mountain, locally known as Red Hill, 10 miles southeast of Shandon. From Shandon follow road up San Juan River 7 miles to E. A. Hall's ranch house, thence go southward over second-class road to station.

Station mark: A bronze tablet set in rock.

Signal: A cedar tree set in ground 26 feet distant; azimuth from station to signal, $198^{\circ}$.

[Latitude $35^{\circ} 36^{\prime} 02.63^{\prime \prime}$. Longitude $120^{\circ} 15^{\prime} 14.04^{\prime \prime}$.]

\begin{tabular}{|c|c|c|c|c|}
\hline To station- & Azimuth. & Back azimuth. & Dista & \\
\hline 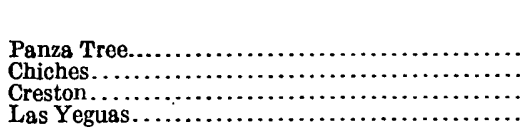 & $\begin{array}{rcc}\circ & \prime & \prime \prime \\
00 & 00 & 06.64 \\
21 & 01 & 05.47 \\
75 & 28 & 23.49 \\
303 & 07 & 48.30\end{array}$ & $\begin{array}{ccc}\circ & \prime & \prime \prime \\
180 & 00 & 06.62 \\
200 & 57 & 41.63 \\
255 & 11 & 07.20 \\
123 & 18 & 10.95\end{array}$ & $\begin{array}{r}\text { Log. meters. } \\
4.3888864 \\
4.3927193 \\
4.2905877 \\
4.5089033\end{array}$ & $\begin{array}{r}\text { Miles. } \\
15.214 \\
15.348 \\
12.132 \\
20.056\end{array}$ \\
\hline
\end{tabular}

\section{SAN JOSE, SAN LUIS OBISPO COUNTY, CAIIF.}

Described in U. S. Coast and Geodetic Survey Report for 1904, App. No. 9, on page 629 , as follows:

"On the top of a peak about 8 miles from the town of San Jose, which is about 30 miles from San Luis Obispo. About 4 miles from San Jose in a canyon is the house of Vidal Garcia. The station is about 4 miles from there by the American Canyon. The station was marked by a concrete pier, a cross on a copper bolt marking the center. Four reference stones were put down, approximately north, south, east, and west, and each 5 feet from the station."

[Latitude $35^{\circ} 18^{\prime} 55.652^{\prime \prime}$. Longitude $120^{\circ} 16^{\prime \prime} 08.225^{\prime \prime}$.]

\begin{tabular}{|c|c|c|c|c|}
\hline Tostation- & Azimuth. & Back azimuth. & Dista & \\
\hline 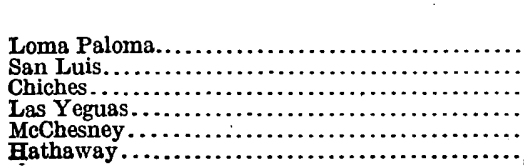 & \begin{tabular}{rcc}
\multicolumn{1}{c}{} & $\prime$ & $\prime \prime$ \\
48 & 38 & 35.01 \\
81 & 13 & 00.12 \\
138 & 54 & 26.26 \\
243 & 44 & 09.98 \\
318 & 58 & 51.20 \\
348 & 12 & 48.04
\end{tabular} & \begin{tabular}{rcc}
\multicolumn{1}{c}{} & $\prime$ & $\prime \prime$ \\
228 & 31 & 23.75 \\
261 & 02 & 52.35 \\
318 & 51 & 34.49 \\
63 & 55 & 01.86 \\
139 & 00 & 11.44 \\
168 & 13 & 58.95
\end{tabular} & $\begin{array}{r}\text { Log. meters. } \\
4.4013924 \\
4.4297266 \\
4.0568484 \\
4.5005937 \\
3.7281113 \\
4.1778311\end{array}$ & $\begin{array}{r}\text { Miles. } \\
15.658 \\
16.714 \\
7.083 \\
19.676 \\
3.322 \\
9.358\end{array}$ \\
\hline
\end{tabular}




\section{SAN LUIS, SAN LUIS OBISPO COUNTY, CALIF.}

Described in U. S. Coast and Geodetic Survey Report for 1904, App. No. 9, page 628, as follows:

"On what is known as the Pine Ridge, in the Santa Lucia or Coast Range, about 6 miles east of San Luis Obispo. This is the highest peak in the vicinity and is covered with pine trees. To reach the station from San Luis Obispo take the main road running toward Arroyo Grande. About 2 miles from the town and just before crossing the first of what are known as the Twin Bridges the peak comes in sight, and can be distinguished by its being somewhat dome-shaped. After crossing the first of these small bridges, turn to the left and continue on to what is known as the Larsen Dairy on the Rancho Corral de Piedra, owned by the Steele Brothers of San Luis Obispo. Follow the creek back of the Larsen Dairy, known as the Canal de Piedra Creek, passing through the place of Patrick McHenry, and about $1 \frac{1}{2}$ miles up the creek from the Larsen Dairy take the trail around to the southeast until you arrive at the main ridge and follow this to the top. The station was marked by a concrete pier, with a cross on a copper bolt in the top of the pier marking the center. The reference stones for the station were put approximately north, south, east, and west, each 4 feet from the station."

[Latitude $35^{\circ} 16^{\prime} 41.102^{\prime \prime}$. Longitude $120^{\circ} 33^{\prime} 40.087^{\prime \prime}$.]

\begin{tabular}{|c|c|c|c|c|}
\hline Tostation- & Azimuth. & Back azimuth. & Dista & ce. \\
\hline 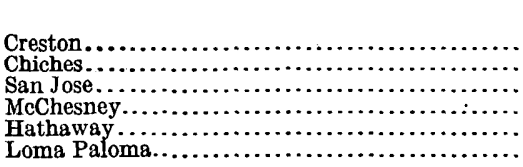 & $\begin{array}{ccc}\circ & \prime & \prime \prime \\
196 & 13 & 25.78 \\
236 & 12 & 08.76 \\
261 & 02 & 52.35 \\
269 & 41 & 30.95 \\
289 & 33 & 29.91 \\
328 & 23 & 33.72\end{array}$ & $\begin{array}{rcc}\circ & \prime & \prime \prime \\
16 & 16 & 52.18 \\
56 & 19 & 25.42 \\
81 & 13 & 00.12 \\
89 & 52 & 58.66 \\
109 & 44 & 47.55 \\
148 & 26 & 29.31\end{array}$ & $\begin{array}{r}\text { Log. meters. } \\
4.5065952 \\
4.3603682 \\
4.4297266 \\
4.4784551 \\
4.4984278 \\
4.1673568\end{array}$ & $\begin{array}{r}\text { Miles. } \\
19.996 \\
14.280 \\
16.714 \\
18.698 \\
19.578 \\
9.135\end{array}$ \\
\hline
\end{tabular}

SIMMLER SCEOOL, SAN LUIS OBISPO COUNTY, CALIF. (Not occupled.)

Station is windmill at Simmler schoolhouse.

Station mark: Pipe in ground under windmill.

Signal: The windmill.

[Latitude $35^{\circ} 21^{\prime} 05.46^{\prime \prime}$. Longitude $119^{\circ} 59^{\prime} 42.03^{\prime \prime}$.]

\begin{tabular}{|c|c|c|c|c|}
\hline Tostation- & Azimuth. & Back azimuth. & Dista & \\
\hline 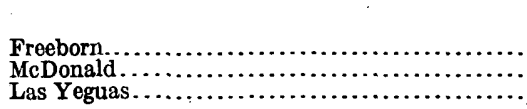 & $\begin{array}{rcc}\circ & \prime & \prime \prime \\
36 & 05 & 58.32 \\
94 & 56 & 27.91 \\
199 & 28 & 08.60\end{array}$ & $\begin{array}{rcc}\circ & \prime & \prime \prime \\
216 & 02 & 56.42 \\
274 & 53 & 01.24 \\
19 & 29 & 29.53\end{array}$ & \begin{tabular}{|r|} 
Log. meters. \\
3.9565127 \\
3.9567018 \\
4.0241725
\end{tabular} & $\begin{array}{r}\text { Miles. } \\
5.622 \\
5.624 \\
6.569\end{array}$ \\
\hline
\end{tabular}

STANLEY, SAN LUIS OBISPO COUNTY, CALIF.

On a high brushy hill, locally known as Stanley Mountain, about 16 miles by road northeast of Santa Maria. From Santa Maria, following the road up Alma Creek about 16 miles to Stanley ranch house, which is at foot of hill. Go up hill eastward about 1 mile to top of steep rocky cliff, thence along ridge another mile to highest point and signal.

Station mark: A copper bolt three-eighths by $1 \frac{1}{2}$ inches cemented in rock flush with ground under signal.

[Latitude $35^{\circ} 04^{\prime} 23.69^{\prime \prime}$. Longitude $120^{\circ} 15^{\prime} 02.42^{\prime \prime}$.]

\begin{tabular}{|c|c|c|c|c|}
\hline To station- & Azimuth. & Back azimuth. & Dista & \\
\hline 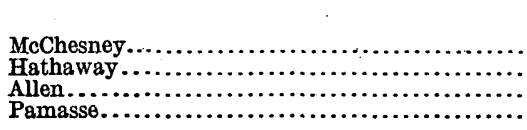 & $\begin{array}{ccc}\circ & \prime & \prime \prime \\
184 & 37 & 20.12 \\
186 & 38 & 16.43 \\
230 & 31 & 55.01 \\
277 & 32 & 37.62\end{array}$ & $\begin{array}{rcc}- & \prime & \prime \prime \\
4 & 38 & 02.21 \\
6 & 38 & 44.53 \\
50 & 37 & 39.48 \\
97 & 36 & 00.76\end{array}$ & $\begin{array}{r}\text { Log. meters. } \\
\text { 4. } 3600442 \\
4.0867225 \\
4.2926926 \\
\text { 3. } 9560414\end{array}$ & $\begin{array}{r}\text { Miles. } \\
14.236 \\
7.587 \\
12.191 \\
5.616\end{array}$ \\
\hline
\end{tabular}




\section{NEVADA. \\ TRIANGULATION STATIONS.}

Coppereid, Dry, Farrell, Lake, Lovelocks, Mazuma, Mill City, and Bochester quadrangles.

\section{CHURCHILL AND HUMBOLDT CODNTIES.}

During the season of $1916 \mathrm{~L}$. F. Biggs extended triangulation over parts of the above-named quadrangles. Positions are based on U. S. Coast and Geodetic Survey stations Augusta, Carson Sink, Granite, and North Augusta, on North American datum.

AUGUSTA, CHURCHILI COUNTY, NEV. (Not occupled.)

Elevation of top of peak, 9,979 feet.

[Latitude $39^{\circ} 32^{\prime} 25.167^{\prime \prime}$. Longitude $117^{\circ} 55^{\prime} 07.696^{\prime \prime}$.]

\begin{tabular}{|c|c|c|c|c|}
\hline To station- & Azimuth. & Back azimuth. & Dista & ince. \\
\hline 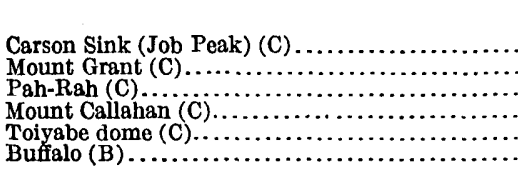 & $\begin{array}{rcc}\circ & \prime & \prime \prime \\
100 & 05 & 22.2 \\
35 & 18 & 40.6 \\
102 & 27 & 16.9 \\
256 & 56 & 55.9 \\
327 & 53 & 19.7 \\
166 & 44 & 51.34\end{array}$ & \begin{tabular}{rcc}
\multicolumn{1}{c}{$\circ$} & $\prime \prime$ \\
279 & 53 & 17.8 \\
214 & 45 & 42.8 \\
281 & 27 & 44.1 \\
77 & 33 & 59.8 \\
148 & 14 & 48.1 \\
346 & 36 & 47.08
\end{tabular} & $\begin{array}{r}\text { Log. meters. } \\
4.4404349 \\
5.1188124 \\
5.1347074 \\
4.9307815 \\
4.9662608 \\
4.8915134\end{array}$ & 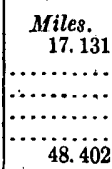 \\
\hline
\end{tabular}

C=U. S. Coast and Geodetic Survey in 1878. $\quad B=$ L. F. Biggs in 1916.

BLACK KNOB, HUMBOLDT COUNTY, NEV.

On a point locally known as Black Knob, 4.5 miles southwest from Rochester.

Station mark: a bronze tablet.

Signal and reference mark: A bottle neck cemented in solid rock and surmounted by a cairn; distant 3.5 feet, true azimuth, $28^{\circ} 39^{\prime}$, from station.

[Latitude $40^{\circ} 14^{\prime} 29.229^{\prime \prime}$. Longitude $118^{\circ} 13^{\prime} 14.795^{\prime \prime}$.]

\begin{tabular}{|c|c|c|c|c|}
\hline Tostation- & Azimuth. & Back azimuth. & Distai & ce. \\
\hline 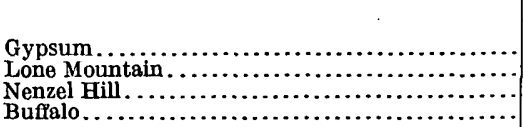 & \begin{tabular}{ccc|}
$\circ$ & $\prime$ & $\prime \prime$ \\
62 & 18 & 58.93 \\
192 & 38 & 39.84 \\
230 & 31 & 34.92 \\
284 & 35 & 18.05
\end{tabular} & \begin{tabular}{rcc}
\multicolumn{1}{c}{} & $\prime$ & $\prime \prime$ \\
242 & 13 & 50.56 \\
12 & 39 & 38.75 \\
50 & 34 & 12.09 \\
104 & 38 & 52.41
\end{tabular} & \begin{tabular}{r|} 
Log. meters. \\
4. 1058191 \\
3. 9923364 \\
3.87171 .49 \\
3.9089173
\end{tabular} & $\begin{array}{r}\text { Miles. } \\
7.928 \\
6.105 \\
4.625 \\
5.038\end{array}$ \\
\hline
\end{tabular}

BUFFALO, HUMBOLDT COUNTY, NEV.

On highest point of Buffalo Peak, 6 miles southeast of Rochester. To reach station follow Packard trail from Rochester to saddle, thence go to left uphill to top of ridge, and along ridge 4 miles.

Station mark: A bronze tablet in solid rock 5-8 feet north of a 1-inch drill hole under signal. True azimuth, $173^{\circ} 58^{\prime}$.

Signal: A juniper tree in small cairn over drill hole. 
[Latitude $40^{\circ} 13^{\prime} 22.885^{\prime \prime}$. Longitude $118^{\circ} 07^{\prime} 42.925^{\prime \prime}$.]

\begin{tabular}{|c|c|c|c|c|}
\hline Tostation- & Azimuth. & Back azimuth. & Dista & ce. \\
\hline 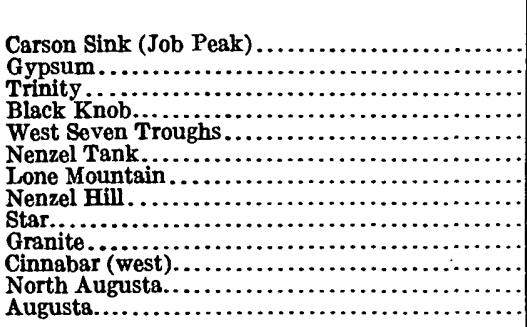 & $\begin{array}{rcc}\circ & \prime & \prime \prime \\
7 & 19 & 07.36 \\
78 & 35 & 13.60 \\
91 & 22 & 08.84 \\
104 & 38 & 52.41 \\
119 & 21 & 36.14 \\
126 & 58 & 02.39 \\
153 & 57 & 49.21 \\
162 & 49 & 16.41 \\
173 & 58 & 08.12 \\
251 & 23 & 04.28 \\
256 & 21 & 09.70 \\
335 & 06 & 41.72 \\
346 & 36 & 47.08\end{array}$ & \begin{tabular}{rcc}
\multicolumn{1}{c}{} & $\prime$ & $\prime \prime$ \\
187 & 15 & 02.20 \\
258 & 26 & 31.05 \\
270 & 58 & 16.61 \\
284 & 35 & 18.05 \\
298 & 54 & 41.39 \\
306 & 50 & 30.91 \\
333 & 55 & 13.56 \\
342 & 48 & 19.11 \\
353 & 56 & 31.35 \\
171 & 46 & 28.78 \\
76 & 33 & 16.01 \\
155 & 19 & 07.00 \\
166 & 44 & 51.34
\end{tabular} & $\begin{array}{r}\text { Log. meters. } \\
4.8548881 \\
4.2908238 \\
4.7196737 \\
3.9089173 \\
4.8282328 \\
4.3145316 \\
4.1122376 \\
3.8507749 \\
4.5246698 \\
4.7328250 \\
4.4365681 \\
4.8176519 \\
4.8915134\end{array}$ & $\begin{array}{r}\text { Miles. } \\
44.488 \\
12.139 \\
32.585 \\
5.038 \\
41.839 \\
12.820 \\
8.046 \\
4.407 \\
20.798 \\
33.587 \\
16.979 \\
40.832 \\
48.402\end{array}$ \\
\hline
\end{tabular}

CARSON SINK, CEURCHILL COUNTY, NEV.

About 20 miles in an easterly direction from town of Stillwater, on highest point of Carson Sink Range of mountains. Station is marked by a copper bolt in a drill hole in solid rock. Also marked a few inches above bolt by a drill hole in a porous rock embedded in the masonry foundation used for mounting the instrument. Station is partly surrounded by a ring wall of rocks about 5.5 meters in diameter. Four drill holes in solid rock are at the following distances and azimuths from station: 5.36 meters, $253^{\circ} 58^{\prime} ; 5.38$ meters, $328^{\circ} 07^{\prime} ; 3.12$ meters, $69^{\circ} 12^{\prime}$; and 1.99 meters, $163^{\circ} 42^{\prime}$. Carson Sink latitude-station pier is 22.68 meters from station in azimuth $230^{\circ} 40^{\prime}$. Vertical-circle station, partly surrounded by a small ring wall of rocks, is 8.13 meters from station in azimuth $39^{\circ} 15^{\prime}$.

Elevation: Top of surface mark, 8,792.6 feet.

[Latitude $39^{\circ} 35^{\prime} 00.232^{\prime \prime}$. Longitude $118^{\circ} 14^{\prime} 05.093^{\prime \prime}$.]

\begin{tabular}{|c|c|c|c|c|}
\hline Tostation- & Azimuth. & Back azimuth. & Dista & nce. \\
\hline 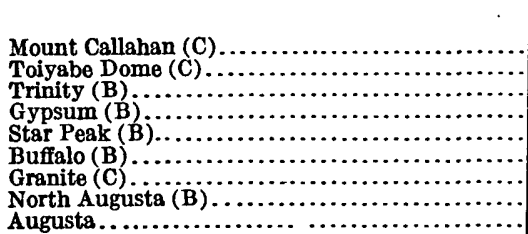 & \begin{tabular}{ccc}
$\circ$ & \multicolumn{1}{c}{,$\prime \prime$} \\
262 & 20 & 28.686 \\
317 & 16 & 00.680 \\
148 & 59 & 53.48 \\
171 & 26 & 13.25 \\
183 & 00 & 25.80 \\
187 & 15 & 02.20 \\
214 & 17 & 32.85 \\
252 & 42 & 23.32 \\
279 & 53 & 17.87
\end{tabular} & \begin{tabular}{rll}
$\circ$ & \multicolumn{1}{c}{ " } \\
83 & 09 & 39.391 \\
137 & 49 & 28.718 \\
328 & 40 & 15.88 \\
251 & 21 & 39.24 \\
3 & 02 & 55.59 \\
7 & 19 & 07.36 \\
34 & 44 & 53.67 \\
72 & 58 & 47.40 \\
100 & 05 & 22.29
\end{tabular} & $\begin{array}{l}\text { Log. meters. } \\
5.04590205 \\
5.05272206 \\
4.9255561 \\
4.8318167 \\
5.0189040 \\
4.8548881 \\
5.0290744 \\
4.5854878 \\
4.4404349\end{array}$ & \begin{tabular}{|r} 
Miles. \\
$\ldots \ldots . .$. \\
\hdashline 52.349 \\
42.186 \\
64.901 \\
44.488 \\
66.439 \\
23.924 \\
17.131
\end{tabular} \\
\hline
\end{tabular}

C=U. S. Coast and Geodetic Survey in 1880. B=L. F. Biggs in 1916.

CINIABAR, WEST, HUHBOLDT COUNTY, NEV. (Not occupied.)

Not described.

[Latitude $40^{\circ} 16^{\prime} 50.400^{\prime \prime}$. Longitude $117^{\circ} 48^{\prime} 58.888^{\prime \prime}$.]

\begin{tabular}{|c|c|c|c|c|}
\hline Tostation- & Azimuth. & Back azimuth. & Dista & \\
\hline 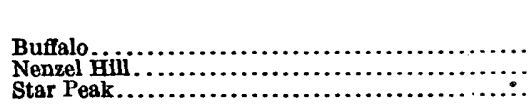 & \begin{tabular}{rcc}
$\circ$ & \multicolumn{1}{c}{ " } \\
76 & 33 & 16.01 \\
90 & 51 & 30.79 \\
131 & 57 & 05.22
\end{tabular} & $\begin{array}{ccc}\circ & , & \prime \prime \\
256 & 21 & 09.70 \\
270 & 38 & 26.71 \\
311 & 43 & 19.85\end{array}$ & $\begin{array}{r}\text { Log. meters. } \\
4.4365681 \\
4.4571332 \\
4.6053665\end{array}$ & $\begin{array}{r}\text { Miles. } \\
16.979 \\
17.803 \\
25.045\end{array}$ \\
\hline
\end{tabular}




\section{GRANITE PEAK, HUMBOLDT COUNTY, NEV. (Not occupled.)}

Elevation of top of peak, 9,779 feet.

[Latitude $40^{\circ} 22^{\prime} 36.653^{\prime \prime}$. Longitude $117^{\circ} 31^{\prime} 31.432^{\prime \prime}$.]

\begin{tabular}{|c|c|c|c|c|}
\hline Tostation- & Azimuth. & Back azimuth. & - Dista & nce. \\
\hline 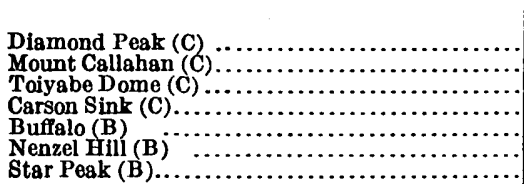 & \begin{tabular}{rcc}
\multicolumn{1}{c}{} & $\prime$ & $\prime \prime$ \\
300 & 31 & 29.9 \\
326 & 16 & 51.7 \\
354 & 59 & 25.3 \\
34 & 44 & 53.7 \\
71 & 46 & 28.78 \\
79 & 16 & 17.83 \\
106 & 42 & 47.17
\end{tabular} & $\begin{array}{lll}\circ & \prime & \prime \prime \\
121 & 37 & 19.5 \\
146 & 39 & 04.0 \\
175 & 06 & 02.4 \\
214 & 17 & 32.9 \\
251 & 23 & 04.28 \\
258 & 51 & 55.06 \\
286 & 17 & 41.42\end{array}$ & \begin{tabular}{|l|} 
Log. meter 8. \\
5. 231148 \\
4. 948721 \\
5. 235650 \\
5. 029074 \\
4. 7328250 \\
4. 7351222 \\
4. 7561888
\end{tabular} & 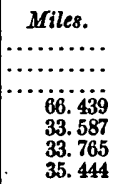 \\
\hline
\end{tabular}

C- United States Coast and Geodetic Survey in 1880. B=L. F. Biggs in 1916.

GPPSUM, HUMBOLDT COUNTY, NEV.

On a range of hills about 10 miles east of Lovelock. To reach station go east from Lovelock to old gypsum mine, thence up ridge on right to top of hill. Animals can be taken to station.

Station mark: A bronze tablet in rock at highest point.

Signal: A juniper tree over tablet.

[Latitude $40^{\circ} 11^{\prime} 16.768^{\prime \prime}$. Longitude $118^{\circ} 21^{\prime} 12.412^{\prime \prime}$.]

\begin{tabular}{|c|c|c|c|c|}
\hline Tostation- & Azimuth. & Back azimuth. & Distar & ce. \\
\hline 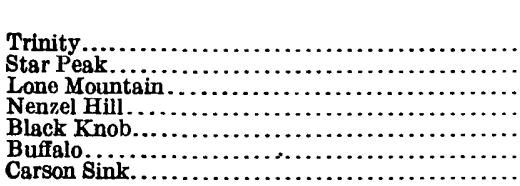 & \begin{tabular}{ccc}
\multicolumn{1}{c}{} & $\prime$ & $\prime \prime$ \\
98 & 35 & 57.55 \\
202 & 40 & 29.24 \\
220 & 50 & 08.16 \\
237 & 53 & 32.15 \\
242 & 13 & 50.56 \\
258 & 26 & 31.05 \\
251 & 21 & 39.24
\end{tabular} & \begin{tabular}{rcc}
\multicolumn{1}{c}{} & \multicolumn{1}{c}{} & $\prime \prime$ \\
278 & 20 & 48.43 \\
22 & 47 & 36.67 \\
40 & 56 & 15.68 \\
58 & 01 & 17.75 \\
62 & 18 & 58.93 \\
78 & 35 & 13.60 \\
171 & 26 & 13.25
\end{tabular} & $\begin{aligned} \text { Log. meters. } \\
\text { 4. } 5272242 \\
\text { 4. } 6053736 \\
\text { 4. } 3124407 \\
\text { 4. } 3032412 \\
\text { 4. } 1058191 \\
\text { 4. } 2908238 \\
\text { 4. } 8318167\end{aligned}$ & $\begin{array}{r}\text { Miles. } \\
20.921 \\
25.045 \\
12.758 \\
12.491 \\
7.928 \\
12.139 \\
42.186\end{array}$ \\
\hline
\end{tabular}

IONE MOUNTAIN, HUMBOLDT COUNTY, NEV.

On highest point of hill west of Limerick Canyon, 3.5 mil es west-of $\cdot$ Rochester.

Station mark: A bronze tablet.

Signal and reference mark: A drill hole surmounted by a cairn 8 feet high, distant 3.2 feet, true azimuth $221^{\circ}$, from station.

[Latitude $40^{\circ} 19^{\prime} 40.035^{\prime \prime}$. Longitude $118^{\circ} 11^{\prime} 43.692^{\prime \prime}$.]

\begin{tabular}{|c|c|c|c|c|}
\hline Tostation- & Azimuth. & Back azimuth. & Dista & \\
\hline 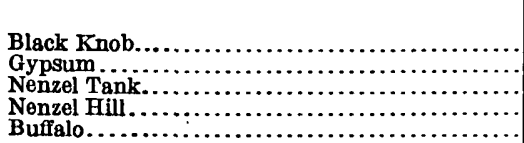 & $\begin{array}{rcc}\bullet & \prime & \prime \prime \\
12 & 39 & 38.75 \\
40 & 56 & 15.68 \\
94 & 02 & 44.34 \\
323 & 30 & 21.21 \\
333 & 55 & 13.56\end{array}$ & $\begin{array}{lcc}\circ & \prime & \prime \prime \\
192 & 38 & 39.84 \\
220 & 50 & 08.16 \\
273 & 57 & 48.20 \\
143 & 31 & 59.59 \\
153 & 57 & 49.21\end{array}$ & \begin{tabular}{r|} 
Log. meters. \\
3. 9923364 \\
4. 3124407 \\
4. 0345421 \\
3. 7811116 \\
4. 1122376
\end{tabular} & $\begin{array}{r}\text { Miles. } \\
6.105 \\
12.758 \\
6.728 \\
\text { 3. } 754 \\
8.046\end{array}$ \\
\hline
\end{tabular}

\section{NENZEL HILL, HUMBOLDT COUNTY, NEV.}

On Nenzel Hill just east of Rochester.

Station mark: A bronze tablet.

Signal: A power pole with targets, distant 23 feet, $283^{\circ} 01^{\prime}$ true azimuth, from station.

Reference marks: Section corner, distant, 280 feet; true azimuth, $314^{\circ} 24^{\prime}$; Rochester mines corner No. 2, distant, 108.8 feet; true azimuth, $71^{\circ} 03^{\prime} 30^{\prime \prime}$. The latter is a round piece of steel $1 \mathrm{inch}$ in diameter, projecting 5 inches above rock in which it is cemented. There is an octagonal steel bar projecting 1.6 feet above surface, cemented in rock 0.9 foot east of round bar. 
[Latitude $40^{\circ} 17^{\prime} 02.554^{\prime \prime}$. Longitude $118^{\circ} 09^{\prime} 11.598^{\prime \prime}$.]

\begin{tabular}{|c|c|c|c|c|}
\hline Tostation- & Azimuth. & Back azimuth. & Dista & \\
\hline 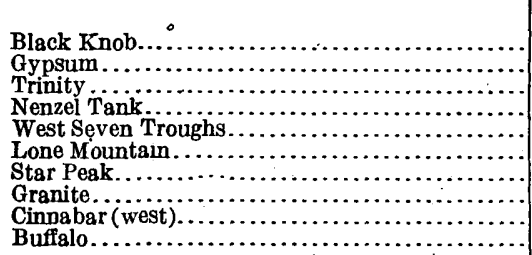 & 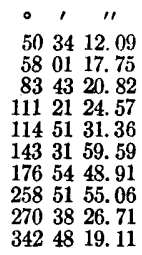 & \begin{tabular}{rcc}
\multicolumn{1}{c}{ ' } & $\prime$ & $\prime \prime$ \\
230 & 31 & 34.92 \\
237 & 53 & 32.15 \\
263 & 20 & 25.00 \\
291 & 14 & 50.17 \\
294 & 25 & 33.07 \\
323 & 30 & 21.21 \\
356 & 54 & 09.55 \\
79 & 16 & 17.83 \\
90 & 51 & 30.79 \\
162 & 49 & 16.41
\end{tabular} & \begin{tabular}{|r|} 
Log. meters. \\
3. 8717149 \\
4. 3032412 \\
4. 7044404 \\
4. 1889881 \\
4. 7950248 \\
3. 7811116 \\
4. 4240360 \\
4. 7351222 \\
4. 4571332 \\
3. 8507749
\end{tabular} & $\begin{array}{r}\text { Miles. } \\
4.625 \\
12.491 \\
31.462 \\
9.601 \\
38.759 \\
3.754 \\
16.496 \\
33.765 \\
17.803 \\
4.407\end{array}$ \\
\hline
\end{tabular}

NENZEL TANK, HUMBOLDT COUNTY, NEV. (Not occupled.)

Not described.

[Latitude $40^{\circ} 20^{\prime} 04.550^{\prime \prime}$. Longitude $118^{\circ} 19^{\prime} 21.260^{\prime \prime}$.]

\begin{tabular}{|c|c|c|c|c|}
\hline Tostation- & Azimuth. & Back azimuth. & Dista & ce. \\
\hline 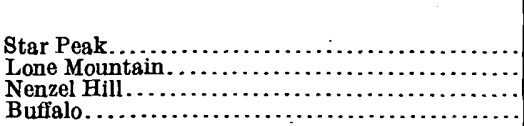 & $\begin{array}{ccc}\circ & \prime & \prime \prime \\
211 & 43 & 04.35 \\
273 & 57 & 48.20 \\
291 & 14 & 50.17 \\
306 & 50 & 30.91\end{array}$ & $\begin{array}{rcc}\circ & \prime & \prime \prime \\
31 & 49 & 00.34 \\
94 & 02 & 44.34 \\
111 & 21 & 24.57 \\
126 & 58 & 02.39\end{array}$ & $\begin{array}{r}\text { Log. meters. } \\
4.3905471 \\
4.0345421 \\
4.1889881 \\
4.3145316\end{array}$ & $\begin{array}{r}\text { Miles. } \\
15.272 \\
6.728 \\
9.601 \\
12.820\end{array}$ \\
\hline
\end{tabular}

NORTH AUGUSTA, HUMBOLDT COUNTY, NEV. (Not occupied.)

No description.

[Latitude $39^{\circ} 41^{\prime} 08.510^{\prime \prime}$. Longitude $117^{\circ} 48^{\prime} 22.386^{\prime \prime}$.]

\begin{tabular}{|c|c|c|c|c|}
\hline To station- & Azimuth. & Back azimuth. & Dist & tance. \\
\hline 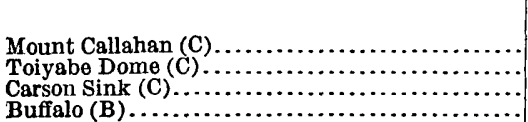 & $\begin{array}{rcc}\circ & \prime & \prime \prime \\
267 & 38 & 45.7 \\
337 & 22 & 39.1 \\
72 & 58 & 47.4 \\
155 & 19 & 07.00\end{array}$ & $\begin{array}{rcc}\circ & \prime & \prime \prime \\
88 & 11 & 34.1 \\
157 & 39 & 53.0 \\
252 & 42 & 23.3 \\
335 & 06 & 41.72\end{array}$ & $\begin{array}{c}\text { Log. meters. } \\
4.866121 \\
5.010504 \\
4.585488 \\
4.8176519\end{array}$ & \begin{tabular}{r} 
Miles. \\
$\ldots . . . . .$. \\
\hdashline... \\
23.924 \\
40.832
\end{tabular} \\
\hline
\end{tabular}

C=U. S. Coast and Geodetic Survey in 1880. B=L. F. Biggs in 1916.

STAR PEAK, HUMBOLDT COUNTY, NEV.

On highest point of Star Peak.

Station mark: A tablet set in rock under monument built over same place at which an old monument was found.

Signal: Center of monument over tablet.

Elevation of top of peak, 9,835 feet.

[Latitude $40^{\circ} 31^{\prime} 22.011^{\prime \prime}$. Longitude $118^{\circ} 10^{\prime} 12.323^{\prime \prime}$.]

\begin{tabular}{|c|c|c|c|c|}
\hline To station- & Azimuth. & Back azimuth. & Dista & nce. \\
\hline 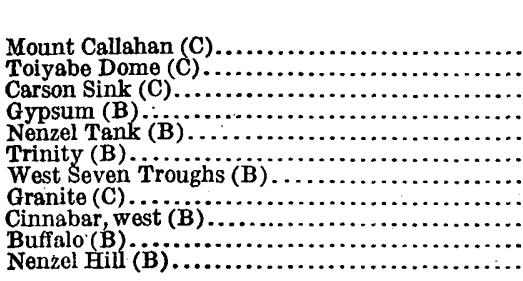 & \begin{tabular}{rcc}
\multicolumn{1}{c}{} & $\prime$ & $\prime \prime$ \\
310 & 33 & 56.9 \\
339 & 14 & 06.9 \\
3 & 02 & 55.7 \\
22 & 47 & 36.67 \\
31 & 49 & 00.34 \\
56 & 45 & 05.26 \\
89 & 41 & 28.04 \\
286 & 17 & 41.42 \\
311 & 43 & 19.85 \\
353 & 56 & 31.35 \\
356 & 54 & 09.55
\end{tabular} & $\begin{array}{ccc}\circ & \prime & \prime \prime \\
131 & 21 & 06.7 \\
159 & 45 & 26.5 \\
183 & 00 & 25.8 \\
202 & 40 & 29.24 \\
211 & 43 & 04.35 \\
236 & 22 & 45.39 \\
269 & 16 & 05.40 \\
106 & 42 & 47.17 \\
131 & 57 & 05.22 \\
173 & 58 & 08.12 \\
176 & 54 & 48.91\end{array}$ & \begin{tabular}{|c|} 
Log. meters. \\
5.138977 \\
5.301670 \\
5.018904 \\
4. 6053736 \\
4.3905471 \\
4.7669007 \\
4.7416937 \\
4.7561888 \\
4.6053665 \\
4.5246698 \\
4.4240360
\end{tabular} & $\begin{array}{l}\text { Miles. } \\
\cdots \cdots . . . . \\
64.001 \\
25.045 \\
15.272 \\
36.329 \\
34.280 \\
35.444 \\
25.045 \\
20.798 \\
16.496\end{array}$ \\
\hline
\end{tabular}

C-U. S. Coast and Geodetic Survey in 1879. B - L. F. Biggs in 1916. 
TRINITY, HUMBOLDT COUNTY, NEV.

On the highest part of a black volcanic mountain about 15 miles west of Lovelocks. Top of hill is about 100 feet long north and south, and station is on south end. To reach station follow Seven Troughs road from Lovelocks about 6 miles to a dim road leading west. Seven Troughs road passes through a saddle 4 miles northeast of point. Station mark: A bronze tablet 7 feet west of signal cairn.

Signal: A cairn 8 feet high.

[Latitude $40^{\circ} 13^{\prime} 57.608^{\prime \prime}$. Longitude $118^{\circ} 44^{\prime} 40.599^{\prime \prime}$.]

\begin{tabular}{|c|c|c|c|c|}
\hline Tostation- & Azimuth. & Back azimuth. & Dista & ce. \\
\hline 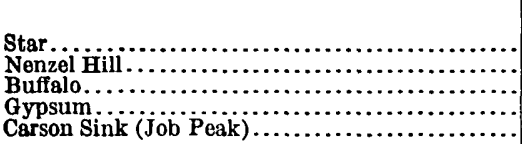 & $\begin{array}{ccc}\circ & \prime & \prime \prime \\
236 & 22 & 45.39 \\
263 & 20 & 25.00 \\
270 & 58 & 16.61 \\
278 & 20 & 48.43 \\
328 & 40 & 15.88\end{array}$ & \begin{tabular}{rcc|}
$\circ$ & $\prime$ & $\prime \prime$ \\
56 & 45 & 05.26 \\
83 & 43 & 20.82 \\
91 & 22 & 08.84 \\
98 & 35 & 57.55 \\
148 & 59 & 53.48
\end{tabular} & \begin{tabular}{|r|} 
Log. meters. \\
4.7669007 \\
4.7044404 \\
4.7196737 \\
4.5272242 \\
4.9255561
\end{tabular} & $\begin{array}{l}\text { Miles. } \\
36.329 \\
31.462 \\
32.585 \\
20.921 \\
52.349\end{array}$ \\
\hline
\end{tabular}

WEST SEVEN TROUGHS, HOMBOLDT COUNTY, NEV. (Not Occupled.)

Not described.

[Latitude $40^{\circ} 31^{\prime} 05.767^{\prime \prime}$. Longitude $118^{\circ} 49^{\prime} 15.847^{\prime \prime}$.]

\begin{tabular}{|c|c|c|c|c|}
\hline To station- & Azimuth. & Back azimuth. & Distar & ce. \\
\hline 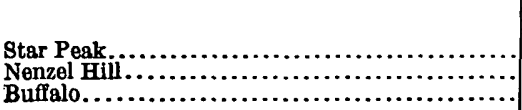 & $\begin{array}{ccc}\circ & \prime & \prime \prime \\
269 & 16 & 05.40 \\
294 & 25 & 33.07 \\
298 & 54 & 41.39\end{array}$ & $\begin{array}{rcc}0 & \prime & \prime \prime \\
89 & 41 & 28.04 \\
114 & 51 & 31.36 \\
119 & 21 & 36.14\end{array}$ & \begin{tabular}{|r|} 
Log. meters. \\
4.7416937 \\
4.7950248 \\
4.8282328
\end{tabular} & $\begin{array}{r}\text { Miles. } \\
34.280 \\
38.759 \\
41.839\end{array}$ \\
\hline
\end{tabular}


